\title{
MÉTODOS DIAGNÓSTICOS DO EQUILÍBRIO NUTRICIONAL DOS MACRONUTRIENTES EM CITROS
}

\section{WAGNER RODRIGUES DOS SANTOS}

Engenheiro Agrícola

Orientador: Prof. Dr. ONDINO CLEANTE BATAGLIA

Dissertação apresentada à Escola Superior de Agricultura "Luiz de Queiroz", Universidade de São Paulo, para a obtenção do título de Mestre em Ciências, Área de Concentração: Fisiologia e Bioquímica de Plantas

PIRACICABA

Estado de São Paulo - Brasil

Outubro - 1997 
Dados Internacionais de Catalogação na Publicação (CIP) DIVISÃO DE BIBLIOTECA E DOCUMENTAÇÃO - Campus "Luiz de Queiroz"/USP

Santos, Wagner Rodrigues dos

Métodos diagnósticos do equilibrio nutricional dos macronutrientes em citros / Wagner Rodrigues dos Santos. - P Piracicaba, 1997.

113 p. : il.

Dissertação (mestrado) - - Escola Superior de Agricultura Luiz de Queiroz, 1997. Bibliografia.

1. Adubação NPK 2. Balanço de nutriente 3. Diagnose foliar 4. Disturbio nutricional de planta 5. DRIS 6. Fruta citrica 7. Macronutirente I. Título

CDD 634.3 


\section{MÉTODOS DIAGNÓSTICOS DO EQUILÍBRIO NUTRICIONAL DOS MACRONUTRIENTES EM CITROS}

WAGNER RODRIGUES DOS SANTOS

Aprovada em: 02.12.1997

Comissão julgadora:

Prof. Dr. Antônio Roque Dechen

Prof. Dr. Ondino Cleante Bataglia

Prof. Dr. Pedro Roberto Furlani
ESALQ/USP

IAC/SAA

IAC/SAA

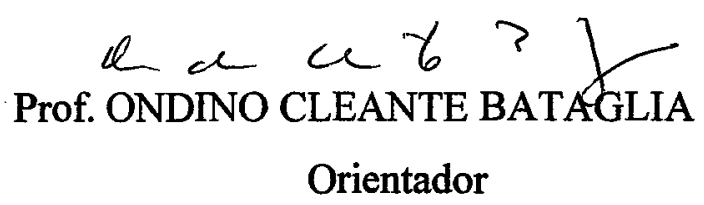


iii

Aiminha espasa Fátima,

Los meus fithos Rafuel e Thiago e

Mosmeuspais

DEDICO 


\section{AGRADECIMENTOS}

- Ao Pesquisador Dr. Ondino Cleante Bataglia, pelo apoio, paciência, confiança e orientação dispensados não só na realização deste trabalho mas também na formação profissional do orientado;

- À FAPESP (Fundação de Amparo à Pesquisa do Estado de São Paulo) pela bolsa de estudo concedida durante o curso sem a qual este trabalho seria inviável;

- À Escola Superior de Agricultura "Luiz de Queiroz" / ESALQ pela oportunidade em participar do curso e pelo agradável e exemplar ambiente acadêmico;

- Ao Instituto Agronômico / IAC em especial a todos os membros da Seção de Fertilidade do Solo e Nutrição de Plantas pela colaboração, apoio técnico e instrumental e facilidades proporcionadas para a condução dos trabalhos de campo e laboratorial;

- Aos Pesquisadores Dr. José Antônio Quaggio, Dr. Pedro Roberto Furlani, Dr. Bernardo van Raij e Dr. Paulo de Souza Gonçalves e ao Professor Dr. Antônio Roque Dechen pelas revisões, críticas e sugestões ao trabalho;

- Aos proprietários dos pomares comerciais que viabilizaram esta pesquisa e tantas outras para o desenvolvimento da atividade citrícola no Estado de São Paulo;

- Ao corpo técnico-administrativo da Cambuhy Empreedimentos Agropecuários Ltda, pelo precioso e confiável banco de dados histórico o qual forneceu o ponto de partida de uma informação tão esperada na área da diagnose nutricional em citros. 


\section{SUMÁRIO}

Página

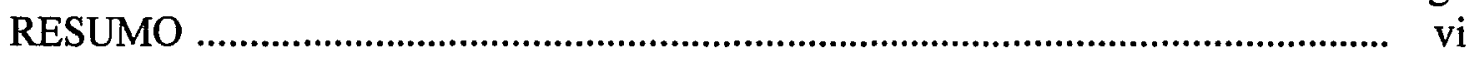

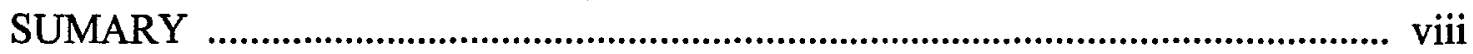

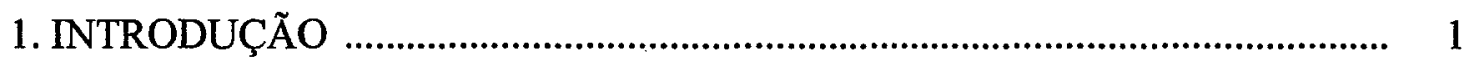

2. REVISÃO DE LITERATURA _.................................................................

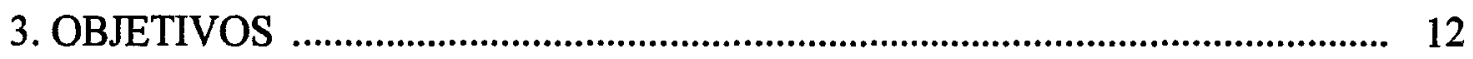

4. MATERIAL E MÉTODOS ......................................................................... 13

4.1. Experimentos de campo ......................................................................... 13

4.2. Amostragem de folha e análise foliar ............................................................... 17

4.3. Pomares comerciais .................................................................................... 17

4.4. Cálculo dos índices DRIS ..................................................................... 18

4.5. Forma de análise dos resultados ............................................................... 19

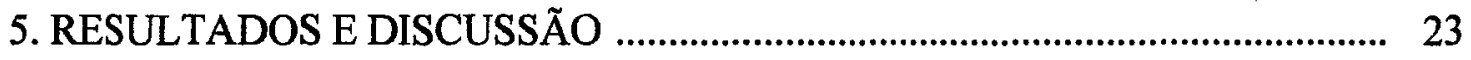

5.1. Definição da população de referência ............................................................. 23

5.2. Definição do procedimento de cálculo ................................................................. 31

5.3. Comportamento dos teores foliares dos macronutrientes .................................. 33

5.4. Padrão nutricional do limoeiro Siciliano ....................................................... 40

5.5. Interpretação do estado nutricional dos citros nos ensaios de adubação com base na população de referência para citros .................................................... 41

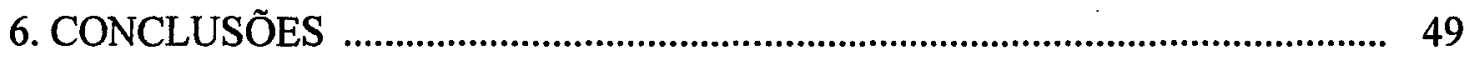

7. REFERÊNCIAS BIBLIOGRÁFICAS ….................................................... 50

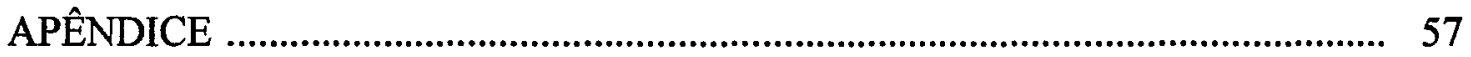




\title{
MÉTODOS DIAGNÓSTICOS DO EQUILÍBRIO NUTRICIONAL DOS MACRONUTRIENTES EM CITROS
}

\author{
Autor: WAGNER RODRIGUES DOS SANTOS \\ Orientador: Prof. Dr. ONDINO CLEANTE BATAGLIA
}

\section{RESUMO}

Com o objetivo de se avaliar o Sistema Integrado de Diagnose e Recomendação, denominado pela sigla DRIS, como critério de interpretação de análise foliar para macronutrientes na cultura de citros, confrontou-se este método com o Critério de Faixas de Suficiência (CFS) através do acompanhamento da resposta em termos de produtividade das plantas cítricas, segundo a diagnose dos métodos.

Para a utilização do DRIS foi necessária a definição da população de referência para os citros, constituindo o ponto mais crítico deste trabalho pela carência de um procedimento padronizado para a seleção das amostras dessa população.

Foram utilizados os resultados de análise foliar de amostras provenientes de uma rede de ensaios de adubação $\mathrm{N}, \mathrm{P}$ e K e de pomares comerciais. Os ensaios de adubação foram instalados em esquema fatorial fracionado $N, P$ e $K,(1 / 2) 4^{3}$, com delineamento experimental de blocos ao acaso, em dois blocos com 16 tratamentos cada, sem repetição, conduzidos durante cinco anos, de 1988 a 1993, em seis locais nas principais regiões citrícolas do Estado de São Paulo. 
O comportamento do teor foliar dos macronutrientes foi relacionado com a produtividade através de análises de correlação, evidenciando-se assim algumas interações entre nutrientes, e entre nutrientes e produtividade.

A interpretação dos índices DRIS referentes aos macronutrientes foi feita considerando que um índice de valor negativo de um determinado nutriente pode estar associado a um fator limitante da produtividade, enquanto que um valor nulo ou positivo está associado à presença do nutriente em um nível suficiente à atividade metabólica não prejudicando a produtividade. Valores extremamente altos não foram verificados quanto ao efeito também prejudicial à produtividade. Também não foi verificado o valor limite de índices negativos próximos de zero que afetam a produtividade.

A comparação de três procedimentos de cálculo de índices DRIS encontrados na literatura e o Critério de Faixas de Suficiência foi feita através da avaliação da eficiência dos métodos diagnósticos do estado nutricional. A eficiência de cada método foi avaliada pelos índices de exatidão, razão $\left(\mathrm{V}^{+}: \mathrm{F}^{+}\right)$e resposta líquida da produtividade.

Desse modo, observou-se uma sensibilidade superior do DRIS sobre o CFS na detecção de plantas com um potencial de resposta de produtividade inibido pelo estado nutricional. $O$ resultado dessa avaliação demonstra a necessidade de ajustes nas faixas de teores dos macronutrientes nos citros e evidencia a superioridade da diagnose pelo método DRIS sobre o Critério de Faixas de Suficiência para orientação da correção do possível desequilíbrio nutricional. $\mathrm{E}$, entre os procedimentos de cálculo baseados no DRIS, o proposto por Jones se destacou principalmente pela sua simplicidade em relação aos demais. 


\title{
DIAGNOSTIC METHODS FOR NUTRITIONAL EQUILIBRIA EVALUATION OF MACRONUTRIENTS IN CITRUS
}

\author{
Author: WAGNER RODRIGUES DOS SANTOS \\ Adviser: Prof. Dr. ONDINO CLEANTE BATAGLIA
}

\section{SUMMARY}

The main objective of this work was to evaluate the Diagnosis and Recommendation Integrated System (DRIS) as a criteria for leaf analysis interpretation of macronutrients in citrus crop. The DRIS method was compared to the Sufficiency Range through yield responses to nutrient application.

The DRIS method required the definition of a reference population for citrus. This was the critical point of this work since there is a lack of standard procedures for sample selection.

The data of foliar analysis, soil analysis and fruit yield derived from a network of six fractional factorial N, P and $\mathrm{K}$ fertilizer experiments. Each experiment was established with 32 plots with yearly rates of $\mathrm{N}(30,100,170$ and $240 \mathrm{~kg} \mathrm{~N} / \mathrm{ha}), P$ $\left(20,60,100\right.$ and $\left.140 \mathrm{~kg} \mathrm{P}_{2} \mathrm{O}_{5} / \mathrm{ha}\right)$ and $\mathrm{K}\left(30,110,190\right.$ and $\left.270 \mathrm{~kg} \mathrm{~K}_{2} \mathrm{O} / \mathrm{ha}\right)$, divided in three applications throughout the raining period. These experiments were conducted from 1988 to 1993 on different soils of the citrus-producing regions of the State of São Paulo, Brazil.

The behaviour of the macronutrient foliar contents was related to fruit yield through correlation analysis to study interactions among nutrients and among nutrients and fruit yield. 
DRIS indices interpretations of macronutrients were based on the assumption that negative values for a nutrient were limitations to yield while null or positive values were associated to sufficiency levels. Extremely high values were not verified as their critical effect on fruit yield.

The comparison of the three computing procedures to calculate DRIS indices found in the literature and the Sufficiency Range was done by performance evaluation of nutritional equilibria diagnostic methods. The efficiency of each method was evaluated by accuracy index, ratio $\left(\mathrm{V}^{+}: \mathrm{F}^{+}\right)$and net yield response.

The results of this study emphasized the need of a better definition for the limits of nutrient contents on citrus leaves to use the Sufficiency Range method. DRIS methods were more responsive than the Sufficiency Range in the detection of potential yield responses. Also, the DRIS was superior to define oriented correction of possible nutritional imbalances.

The procedure proposed by Jones was selected among the methods of calculating DRIS indices because of its simplicity and ease of use. 


\section{INTRODUÇÃO}

A competitividade na oferta vem acentuando a busca da eficiência produtiva com uma maior preocupação no planejamento da produção e controle dos custos de produção. Pois, a sobrevivência do produtor depende da habilidade com que este se adapta ao processo produtivo ao preço do produto a fim de conseguir manter a relação benefício/custo a maior possível.

Uma adubação adequada começa com a análise de solo, que permite avaliar a fertilidade através dos valores de saturação de bases e dos teores de fósforo, potássio, cálcio, magnésio e micronutrientes.

Por exemplo, um teor muito baixo de fósforo $\left(0 \mathrm{a} 6 \mathrm{mg} \mathrm{dm}^{-3}\right)$ ou de potássio ( 0 a $0,7 \mathrm{mmol}_{c} \mathrm{dm}^{-3}$ ) representa, caso o nutriente não seja aplicado, uma redução de no mínimo $30 \%$ da produtividade máxima possível.

Outra característica referente à fertilidade do solo é a lenta correção de situações como a baixa fertilidade. Mesmo bem adubado a resposta em termos de produtividade não será a níveis compativeis aos insumos empregados, pelo menos a curto prazo. Então, a recomendação técnica é elevar os parâmetros da análise de solo para pelo menos à faixa de teores ou valores médios, correspondendo à expectativa moderada de produtividade.

A adubação também recebe orientação direta de indicadores do estado nutricional como os teores foliares dos nutrientes, mais precisamente no caso da adubação nitrogenada. Isto mostra a importância de uma maior quantidade de ferramentas para uma correta recomendação do tipo e da quantidade de adubo a ser usado. 
Quanto a análise química de solo o que se tem de informação já possibilita correções nos níveis de fertilidade com ganhos de produtividade. No entanto, para a análise química do tecido vegetal em folhas, o que se tem são teores foliares médios dos nutrientes classificados em faixas ou valores nos quais se tem níveis de produtividade diferenciados. Esses valores vem sendo utilizados largamente na citricultura a fim de detectar alguma deficiência nutricional. A avaliação de teores, pelo método denominado "Critério de Faixas de Suficiência", não leva em consideração a interação entre nutrientes no interior da planta o que pode induzir a correções errôneas do nivel do nutriente no solo.

Esta lacuna na interpretação de resultados de análise química foliar pode ser minimizada com a utilização de relações entre nutrientes o que teoricamente minimiza também a influência da idade do tecido, época de amostragem entre outros fatores que alteram os teores dos nutrientes. O Sistema Integrado de Diagnose e Recomendação, do inglês Diagnosis and Recommendation Integrated System (DRIS), apresenta estas características o que o torna uma ferramenta auxiliar de grande potencial na avaliação do estado nutricional.

A eficiência dos métodos tem despertado interesse por ser responsável pela orientação na correção do estado nutricional, logo, pelo uso racional de insumos, no caso os fertilizantes. Então, uma diagnose mal sucedida pode por um lado agravar o desequilíbrio nutricional com reflexo direto na produtividade, ou pelo lado econômico pode-se ter uma aplicação desnecessária sem uma resposta positiva da produtividade, somente aumentando os custos de produção.

O presente trabalho visa dar indicação dos melhores procedimentos para a diagnose de desequilíbrios nutricionais e recomendação de correção desses desequilíbrios. 


\section{REVISÃO DE LITERATURA}

$\mathrm{Na}$ agricultura moderna e competitiva não há mais espaço para improvisações e perdas de colheitas ou reduções de lucros por causa de falhas de controle de fatores de produção perfeitamente previsíveis. De um modo geral este é o caso da questão relacionada com a nutrição mineral das plantas. Assim Bataglia et al. (1996) retratam a importância do conhecimento tecnológico no controle eficiente de fatores de produção.

A nutrição mineral de plantas é responsável em muito no desempenho qualitativo e quantitativo de um vegetal (Woods \& Villiers, 1992), sendo possível prevenir insucessos devido a deficiências ou excessos de nutrientes pela correção dos solos usando a análise química como critério para a recomendação de corretivos e fertilizantes. Atualmente, é possivel usar a própria planta como objeto de diagnóstico (Bataglia et al.,1992 e Bataglia et al.,1996).

A folha como um dos órgãos de maior atividade metabólica, pode apresentar, na maioria das vezes, alterações fisiológicas devidas a distúrbios nutricionais. Por esse motivo é que quase sempre os diagnósticos nutricionais das plantas são feitos através das folhas pela técnica que de forma ampla se denomina como diagnose foliar (Bataglia et al.,1996 e Terblanche \& Du Plessis, 1992).

A diagnose foliar pode ser feita por meio da observação visual de sintomas de distúrbios nutricionais, recebendo a denominação de diagnose visual, ou através de procedimentos mais sofisticados envolvendo por exemplo a análise química das folhas. Em ambos os casos há necessidade de se observarem determinados princípios para que os resultados possam ser devidamente interpretados e levem a recomendações 
com resultados econômicos para os produtores (Bataglia et al.,1996 e Malavolta et al., 1989).

O uso correto da diagnose foliar como método de avaliação do estado nutricional da planta depende do conhecimento das limitações da técnica. Até hoje, de acordo com as ponderações de Jones et al.(1991) é preciso questionar a confiabilidade dos dados, a utilização de conceitos de relações e balanços de nutrientes, o efeito de cultivares e o efeito de concentrações variáveis de nutrientes alterando processos fisiológicos.

A correta interpretação dos resultados de uma análise química do tecido vegetal depende de muita experimentação visando o estabelecimento de padrões de calibração que reflitam o estado nutricional das plantas. Entre os critérios mais usados para diagnóstico são citados o nível crítico, faixas de concentração, e o sistema integrado de diagnose e recomendação (DRIS) (Bataglia et al.,1996 e Malavolta et al., 1989).

Bataglia et al.(1996) alertam sobre a necessidade do conhecimento dos fatores que afetam a concentração dos nutrientes nas folhas. Citam também, Munson \& Nelson (1973), que considerando a multiplicidade de fatores que influenciam o crescimento e a produtividade de uma planta, é até surpreendente que as relações entre a análise de plantas, suprimento de nutrientes e produção sejam tão válidas.

A avaliação do estado nutricional e o tratamento de sintomas agudos de distúrbios nutricionais são referenciados à interpretação dos resultados. Esta avaliação nada mais é que uma comparação do resultado da amostra em análise com os valores obtidos pela análise de uma amostra com o parâmetro conhecido, que se deseja otimizar, como a produtividade e ou a qualidade(Bataglia et al.,1996 e Woods \& Villiers, 1992).

A premissa básica de que a otimização da condição vegetativa da cultura é essencial para a melhoria da produtividade, impõe à interpretação dos resultados, não só a avaliação do estado nutricional das plantas, como também, a orientação dos tratamentos de sintomas agudos de distúrbios nutricionais. Contudo, é essencial a existência de valores de referência para possibilitar a comparação dos dados 
da amostra em análise (Bataglia et al.,1996 e Malavolta et al., 1989). A princípio esses valores referenciais deveriam ter aplicação universal, entretanto, na prática há uma forte relação com particularidades ambientais (Gallo et al., 1977; Haag, 1987 e Hiroce, 1987), de modo que os valores de referência mais fiéis necessitam ser obtidos nas condições locais, embora na inexistência de um padrão local, alguma extrapolação seja possível (Beaufils, 1971 e Beaufils, 1973).

Os valores de referência utilizados na avaliação do estado nutricional são obtidos segundo a característica da planta que se deseja a expressão ótima. No geral, o desejado é maximizar a produção. Então, podem-se citar os critérios de interpretação baseados no nível crítico e em faixas de valores correspondentes a zonas de deficiência aguda, deficiência latente, ótima, consumo de luxo e excesso (Bataglia et al.,1996 e Malavolta et al., 1989). Esses dois critérios consideram apenas as concentrações dos nutrientes na matéria seca. Outro critério que vem se popularizando é o Sistema Integrado de Diagnose e Recomendação (Beaufils, 1971 e Beaufils, 1973), que utiliza o conceito de balanço de nutrientes e é menos sujeito a interferências ambientais e dos sistemas de amostragem das plantas.

"O nível crítico corresponde à concentração na folha abaixo do qual a taxa de crescimento, a produção ou a qualidade são significativamente diminuídas". Esta definição em Bataglia et al.(1996) evidencia o caráter, um tanto quanto subjetivo, do ponto considerado crítico, ou seja, para Ulrich \& Hills (1967) o nível crítico é a concentração correspondente a produção de $95 \%$ relativa a ótima, enquanto Gallo et al. (1965) estabeleceram níveis críticos para milho com base numa produção relativa de $80 \%$.

A relação entre o teor foliar do nutriente e a produção relativa apresenta, para uma estreita faixa de teores foliares, uma grande amplitude de variação na produção relativa. Este fato é importante na determinação do valor crítico, que é muito sensível, isto é, uma pequena variação no valor considerado pode conduzir a produtividade esperada a valores indesejáveis.

Outro ponto nesse critério susceptível a críticas refere-se ao fato de que não só deficiências nutricionais resultam em redução da produção, crescimento ou 
qualidade, mas também altas concentrações. Então, Okhi (1987) aprimorou o conceito de nível crítico como sendo a concentração que provoca uma redução de $10 \%$ na produção máxima, surgindo assim o conhecido nível crítico de deficiência e nível crítico de toxidez.

A essência do critério de faixas de concentração ou faixas de suficiência é a não existência de um ponto de produção ótima, mas sim de uma faixa na qual a produção se situa a níveis ótimos. Logo, é de se esperar que o fornecimento de nutrientes esteja em niveis adequados o que promove uma concentração do nutriente nos tecidos também considerada adequada. Nesta primeira aproximação do que ocorre com o teor foliar temos três faixas: uma faixa de deficiência, uma adequada e uma de excesso (Bataglia et al.,1996). Na Tabela 1 estão apresentadas as características de cada faixa com as considerações feitas por Cottenie (1980).

Tabela 1. Características de cada faixa de concentração usados na avaliação do estado nutricional pelo método do Critério de Faixas de Suficiência.

Faixa Características

\begin{tabular}{ll}
\hline $\begin{array}{l}\text { Excesso ou toxidade } \\
\text { Consumo de luxo }\end{array}$ & $\begin{array}{l}\text { Decréscimo da produção possivelmente com sintomas visuais } \\
\text { Bom crescimento mas com acúmulo interno de nutrientes e } \\
\text { possiveis interações }\end{array}$ \\
Ótimo de nutrição & $\begin{array}{l}\text { Com crescimento e geralmente boa qualidade } \\
\text { Deficiência latente }\end{array}$ \\
& $\begin{array}{l}\text { Sem sintomas visuais, mas que apresenta resposta à aplicação } \\
\text { de fertilizante com aumento de produtividade e ou melhoria } \\
\text { na qualidade }\end{array}$ \\
Deficiência aguda & $\begin{array}{l}\text { Sintomas visuais e efeito direto da fertilização e aplicação } \\
\text { foliar }\end{array}$
\end{tabular}

Fonte: Cottenie (1980).

O desenvolvimento da representação gráfica dos teores dos nutrientes, foi mostrado por Malavolta et al.(1989). Inicialmente, através das ordenadas triangulares pretendia-se descrever o equilíbrio nutricional de três nutrientes de uma amostra 
representativa de uma população altamente produtiva, através da localização da participação percentual de cada um dos nutrientes no interior de um triângulo equilátero.

A seguir, o Fertigrama que originalmente era utilizado para avaliar a fertilidade do solo foi adaptado à avaliação do estado nutricional a partir de resultados de análise foliar por Malavolta \& Romeu, citados por Malavolta et al. (1989), o que pode ser considerado como um aprimoramento das Ordenadas Triangulares. Os raios eqüidistantes de uma circunferência são os eixos representando o teor do nutriente na folha, a partir do centro. Após localizar cada teor foliar na amostra em diagnose unem-se os pontos formando um polígono. A evolução do estado nutricional pode ser verificado através da representação, no mesmo gráfico, dos teores foliares referentes a vários anos. $O$ desequilíbrio do estado nutricional, deficiência ou excesso, é constatado pela irregularidade do polígono, pontas e reentrâncias acentuadas.

$\mathrm{O}$ passo seguinte para a melhoria da diagnose foi a representação, no diagrama circular ou no gráfico de barras, dos teores encontrados na amostra como porcentagem em relação a teores considerados adequados, normais ou ótimos. $\mathrm{O}$ comprimento do raio ou da barra corresponde a porcentagem encontrada.

$\mathrm{O}$ fato de que os teores encontrados em plantas consideradas normais podem extender-se muito nas faixas utilizadas para indicar o estado nutricional, segundo a classificação proposta, pode dificultar a diagnose, pois esta elasticidade varia de nutriente para nutriente. Para minimizar este efeito sobre a avaliação foi utilizado o coeficiente de variação $(\mathrm{CV})$, para ponderar a variabilidade associada com a composição das plantas normais (Malavolta et al., 1989).

A evolução da diagnose do estado nutricional tendeu a compreender a nutrição de plantas como um complexo sistema bioquímico, onde o inter-relacionamento de inúmeros fatores internos e externos à planta é que definem o desempenho produtivo do vegetal (Beaufils, 1971; Marshner, 1986; Mengel \& Kirkby, 1978; Salisbury \& Ross, 1991 e Taiz \& Zaiger, 1991).

O equacionamento desta situação poderia ser feito através de curvas de regressão. Contudo a simulação do efeito de cada fator condicionante, por exemplo, da produtividade é quase que infactível. Beaufils (1971), através da diagnose fisiológica, 
demonstrou a viabilidade de se avaliar os fatores que atuam sobre a planta em especial os níveis de $\mathrm{N}, \mathrm{P}$ e $\mathrm{K}$ em milho. Em 1973, propôs um procedimento de cálculo de indices que refletissem as relações entre diversos fatores, levando em consideração a variabilidade inerente ao fator numa população não submetida a condições anormais (População não-anormal).

O critério de interpretação do DRIS - Diagnosis and Recommendation Integrated System (Beaufils, 1973), é menos sujeito à variações ambientais, idade e diferentes combinações copa $x$ porta-enxerto como as estudadas por Hiroce (1987) e Gallo et al.(1977), quando utilizado na avaliação do estado nutricional de citros. Isso porque o método baseia-se para o cálculo de índices de cada nutriente no relacionamento dele com os demais e comparando essas relações com as relações médias, também denominadas de relações ótimas, de uma população de plantas de alta produtividade, a norma, população padrão ou população de referência, assim denominada por Jones (1986). Desta forma diferencia-se do método clássico no qual a interpretação é feita comparando-se os resultados analíticos de cada nutriente com valores tabelados (Chapman, 1966), não possibilitando avaliar qual o nutriente mais limitante no caso de dois ou mais nutrientes encontrarem-se abaixo dos níveis críticos (Malavolta, 1989). Para cada nutriente, tem-se que para índices com valores negativos a indicação de deficiência e positivos excessos, enquanto que valores próximos de zero indicam uma nutrição equilibrada. Além disso, é possível identificar uma sequiência de nutrientes mais e menos limitantes da produtividade.

O DRIS, inicialmente, desenvolvido para seringueira (Beaufils, 1973) tem-se mostrado útil para outras plantas, havendo já trabalhos desenvolvidos, por exemplo, com milho (Sumner, 1977; Elwali et al., 1985), alfafa (Walworth et al., 1986), trigo (Amundson \& Koehler, 1987), chá (Lee, 1981), laranjeira (Beverly et al., 1984).

No Brasil, o desenvolvimento da técnica é ainda incipiente, com poucos trabalhos publicados, como o de Zambello Jr.(1980) com cana de açúcar e o de Bataglia \& Santos (1990) com seringueira. Apesar disso, ela vem sendo divulgada em diversos simpósios (Bataglia \& Dechen, 1986; Malavolta \& Malavolta, 1988) e está sendo testada em alguns trabalhos de pesquisa com cafeeiro (Wadt \& Gonçalves, 1997), 
limoeiro siciliano (Creste, 1996), mamoeiro (Costa, 1995), pimenteira-do-reino (Oliveira et al., 1996), videira (Guilherme, 1995 e Gergoletti, 1995) e soja (Leandro et al., 1996).

Wood \& Villiers (1992) associaram a resposta em termos de quantidade e qualidade para definir a população de interesse, para tal foram descartadas as informações das amostras representativas de árvores com produção fora do intervalo dos 100 aos $500 \mathrm{~kg}$ de frutas por árvore. Dessas amostras com produtividade aceitável foram selecionadas as amostras com peso de fruta na faixa dos 170 aos $400 \mathrm{~g}$ por fruta para a definição da subpopulação de referência e na faixa dos 100 aos $170 \mathrm{~g}$ por fruta uma subpopulação de contraste, para permitir a escolha das relações mais sensíveis no cálculo dos índices DRIS.

A separação de nutrientes limitantes de não-limitantes foi conduzida para soja no trabalho de Hallmark et al. (1987b), no qual foi elaborada a avaliação dos teores de $\mathrm{P}$ e $\mathrm{K}$ comparando-se também com a técnica do nível crítico e ainda com a com a finalidade de recomendação' de adubação.

Hallmark et al. (1987a) compararam dois métodos DRIS, o que usa o procedimento proposto por Beaufils (1973) com o proposto por Jones (1981), quanto a exatidão da diagnose de deficiências de fósforo e potássio em soja (Glycine max. L.), sendo que foi acrescentado mais um fator no cálculo dos índices, a matéria seca. Significando a participação do teor do nutriente no cálculo do índice DRIS. Esta alteração foi denominada de M-DRIS ou DRIS modificado. Nestas condições o método DRIS - Jones foi mais exato que o tradicional para o nutriente mais deficiente como também para o segundo. Neste mesmo trabalho os autores já fazem referência a um protocolo de comparação de métodos diagnósticos.

Beverly \& Hallmark (1992) propuseram um protocolo para permitir a avaliação de métodos diagnóstico através das diagnoses corretas em função da presença de resposta da produtividade. A verificação da diagnose fica condicionada a existência da situação de fornecimento do nutriente quer pelo estudo histórico da parcela ou de ensaios fatoriais de adubação no qual se tem as combinações de doses de fornecimento do nutriente. 
Segundo esses mesmos autores, a produtividade reduzida pode ser devido a uma baixa disponibilidade de um determinado nutriente. Essa disponibilidade no solo tem origem no baixo fornecimento do nutriente via adubação, por exemplo, ou pela interação do nutriente com outros fatores e até mesmo com outros nutrientes. Isso pode conduzir a diagnoses errôneas, em que o fornecimento do nutriente deficiente não resulta em resposta em termos de produtividade, ou seja, a aplicação é desnecessária com implicação econômica direta, além de poder agravar o desequilíbrio entre os nutrientes. Então, pode-se esperar da diagnose quatro situações possíveis, classificadas na Tabela 2.

Tabela 2. Classificação e codificação da diagnose quanto a resposta em termos de produtividade.

Diagnose Resposta Codificação* Implicação

\begin{tabular}{|c|c|c|c|}
\hline Deficiente & Sim & $\mathrm{V}^{+}$ & Fertilizante aplicado com aumento de produtividade \\
\hline Deficiente & Não & $\mathrm{F}^{+}$ & Fertilizante aplicado desnecessariamente \\
\hline Adequado & Sim & $F^{-}$ & $\begin{array}{l}\text { Fertilizante não aplicado, oportunidade de aumento } \\
\text { de produtividade perdida }\end{array}$ \\
\hline Adequado & Não & $\mathrm{V}^{-}$ & $\begin{array}{l}\text { Fertilizante não aplicado, a produtividade não } \\
\text { apresenta resposta positiva com o fornecimento do } \\
\text { nutriente }\end{array}$ \\
\hline
\end{tabular}

Fonte: Beverly \& Hallmark (1992)

Segundo os mesmos autores, a eficiência de um método diagnóstico é avaliada por três índices quantitativos: a exatidão da diagnose $\left(\mathrm{V}^{+}+\mathrm{V}^{-}\right)$, a razão de diagnoses positivas $\left(\mathrm{V}^{+}: \mathrm{F}^{+}\right)$e a resposta de produtividade líquida (o termo resposta é usado para uma variação mínima de $10 \%$ da produtividade quando não se tem quantificada a variação da produtividade). Esses índices são calculados a partir dos valores percentuais do total de casos em diagnose.

\footnotetext{
" A codificação no trabalho original $\mathrm{T}$ de "True" foi traduzida para o português para uma melhor compreensão passando a ser denominada $\mathrm{V}$ de "Verdadeiro", sendo que o $\mathrm{F}$ permaneceu sem alteração pois passou de "False" para "Falso".
} 
Beverly (1992) aplicou o protocolo proposto por Beverly \& Hallmark (1992) na comparação do Critério de Faixas de Suficiência, DRIS, DRIS com transformações logarítmicas, concentração com transformações logarítmicas e dados de concentração interpretados de forma similar ao procedimento de Kenworthy, obtendo para as condições do trabalho uma superioridade das diagnoses pelo Critério de Faixas de Suficiência sobre os demais métodos.

Estes trabalhos confirmam a necessidade da utilização de ferramentas complementares à análise de solo, para o diagnóstico mais seguro das necessidades de adubação, e racionalização do uso de fertilizantes na agricultura. 


\section{OBJETIVOS}

Geral: avaliar o DRIS como critério de interpretação de resultados de análises de folhas de plantas cítricas, e compará-lo com o critério baseado em faixas de concentrações.

Específico: definir a população normal de plantas para a citricultura paulista. 


\section{MATERIAL E MÉTODOS}

O projeto utilizou os dados coletados de experimentos de campo de longa duração, conduzidos pela Seção de Fertilidade do Solo e Nutrição de Plantas (SFSNP) do Instituto Agronômico (IAC), resultados de análises foliares de pomares comerciais e "software" especificamente desenvolvido para o DRIS, em projeto anterior de iniciação científica apoiado pela Fundação de Amparo à Pesquisa do Estado de São Paulo (FAPESP).

\subsection{Experimentos Fatoriais $N, P$ e $K$}

Seis experimentos de campo foram conduzidos em diferentes solos nas principais regiões citrícolas do Estado de São Paulo. Os locais experimentais são caracterizados na Tabela 3.

Tabela 3. Informações complementares sobre os ensaios.

\begin{tabular}{lcllcl}
\hline Localização & Plantio & Copa & Porta-enxerto & Espaçamento & Tipo de solo \\
\hline & & & & $---m---$ & \\
Araraquara & 1983 & Lar.Pera & Lim.Cravo & $\mathbf{7 , 0 \times 4 , 0}$ & LatossoloVerm.EscuroDistrófico \\
Botucatu & 1982 & Lim.Siciliano & Tang.Volkam. & $\mathbf{8 , 0 \times 6 , 0}$ & LatossoloVerm.EscuroDistrófico \\
Matão & 1981 & Lar.Valência & Lim.Cravo & $\mathbf{8 , 0 \times 6 , 0}$ & PodzólicoVerm.AmareloEutrófico \\
MonteAzul & 1982 & Lar.Pera & Lim.Cravo & $\mathbf{7 , 5 \times 6 , 5}$ & PodzólicoVerm.AmareloEutrófico \\
Olímpia & 1981 & Lar.Natal & Lim.Cravo & $\mathbf{8 , 0 \times 6 , 0}$ & LatossoloVerm.EscuroDistrófico \\
Pirassununga & 1984 & Lar.Pera & Tang.Cleópatra & $\mathbf{7 , 5 \times 4 , 0}$ & LatossoloVermelhoAmareloÁlico \\
\hline
\end{tabular}

O delineamento experimental foi de blocos ao acaso em esquema fatorial fracionado (1/2) $4^{3}$ com dois blocos, sem repetições, num total de 32 tratamentos, como proposto por Colwell (1978), com tratamentos ajustados por Andrade 
\& Noleto (1986) para permitir cálculos das interações de primeira ordem, isto é, assegurar a ortogonalidade dos tratamentos.

As doses estabelecidas para a aplicação em área total foram: 30,100 , 170 e $240 \mathrm{~kg} / \mathrm{ha}$ de $\mathrm{N} ; 20,60,100$ e $140 \mathrm{~kg} / \mathrm{ha}$ de $\mathrm{P}_{2} \mathrm{O}_{5}$ e $30,110,190$ e $270 \mathrm{~kg} / \mathrm{ha}$ de $\mathrm{K}_{2} \mathrm{O}$, usando como fonte dos nutrientes os fertilizantes nitrocálcio, superfosfato triplo e cloreto de potássio, respectivamente, distribuídos em área total.

Os fertilizantes foram parcelados em três aplicações nos meses de set/out, dez/jan e mar/abr. As plantas foram pulverizadas duas vezes no ano com solução contendo os micronutrientes $\mathrm{Zn}, \mathrm{Mn}$ e $\mathrm{B}$. No início do experimento, todas as áreas tiveram a acidez corrigida para saturação em bases de $70 \%$.

As parcelas foram constituídas por vinte plantas, sendo as seis centrais utilizadas para protocolos e avaliação de produção. Com esse número de plantas por parcelas foi possível estabelecer linhas duplas de bordadura entre os diversos tratamentos.

$\mathrm{Na}$ Tabela 4, são apresentadas as informações referentes às parcelas experimentais comuns a todos os locais, quanto a distribuição dos tratamentos aos blocos e as doses de $\mathrm{N}, \mathrm{P}$ e $\mathrm{K}$ de cada tratamento.

Tabela 4. Descrição geral dos tratamentos com as quantidades totais anuais dos nutrientes aplicados.

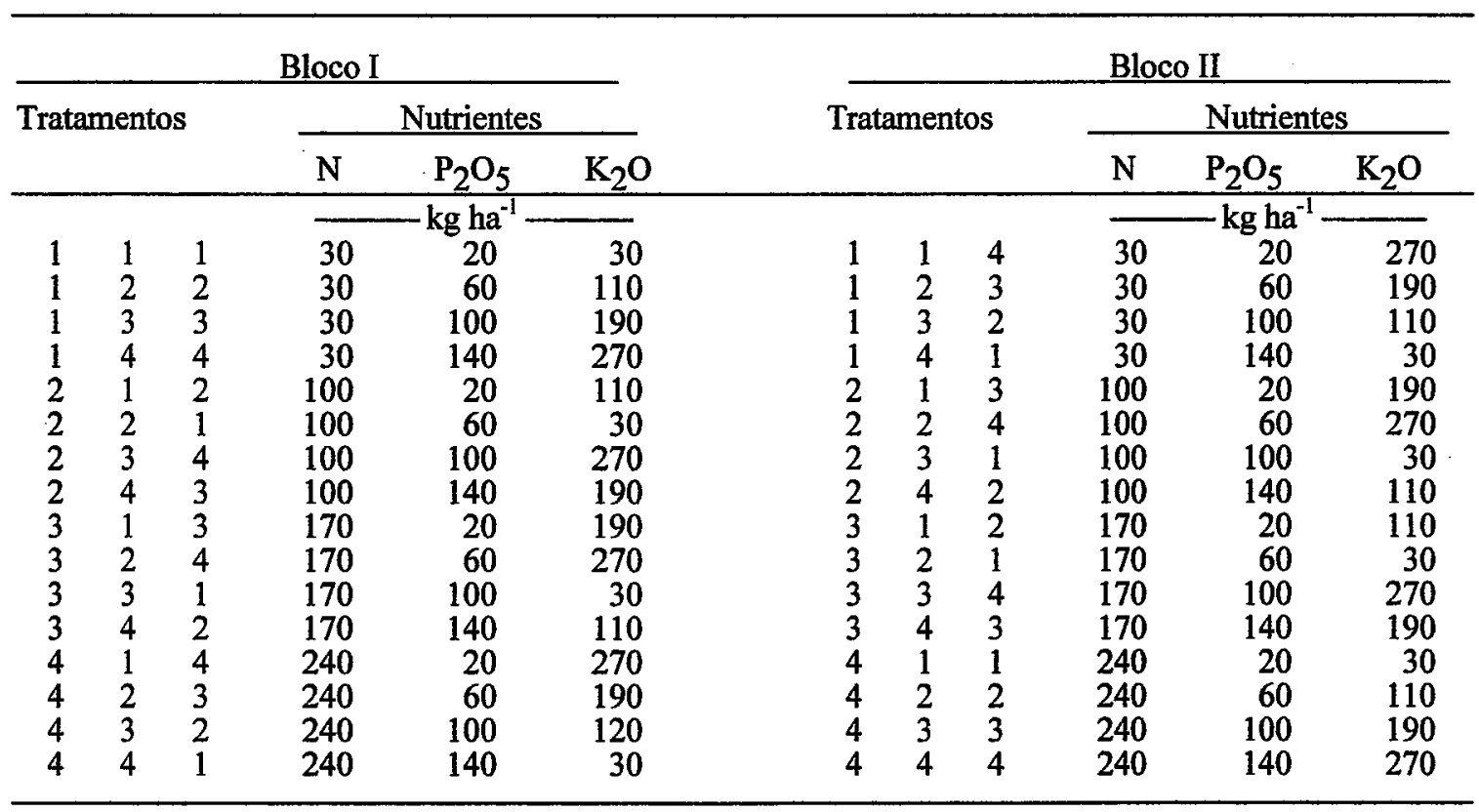


As condições edáficas iniciais de cada local, em duas profundidades, foram agrupadas na Tabela 5, permitindo a caracterização do local experimental.

Tabela 5. Resultados de análise de solo em duas profundidades, antes da aplicação de fertilizantes no primeiro ano.

\begin{tabular}{|c|c|c|c|c|c|c|c|c|c|c|}
\hline \multirow{3}{*}{ Local } & \multirow{3}{*}{$\begin{array}{c}\text { Camada } \\
\text { do } \\
\text { solo } \\
\end{array}$} & \multirow{3}{*}{$P_{\text {resina }}$} & \multirow{3}{*}{$\begin{array}{c}\mathrm{pH} \\
\mathrm{em} \\
\mathrm{CaCl}_{2}\end{array}$} & \multirow{3}{*}{$\begin{array}{l}\text { Matéria } \\
\text { Orgânica }\end{array}$} & \multicolumn{3}{|c|}{ Cátions Trocáveis } & \multirow{3}{*}{$\mathrm{H}+\mathrm{Al}$} & \multirow{3}{*}{ CTC } & \multirow{3}{*}{$\begin{array}{c}\text { Saturação } \\
\text { de } \\
\text { bases }\end{array}$} \\
\hline & & & & & & & 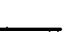 & & & \\
\hline & & & & & $\mathbf{K}$ & $\mathrm{Ca}$ & $\mathrm{Mg}$ & & & \\
\hline & $\mathrm{cm}$ & $\mathrm{mg} \mathrm{dm}^{-3}$ & & $\mathrm{~g} \mathrm{dm}^{-3}$ & - & $\longrightarrow$ & $\mathrm{mol}_{\mathrm{c}}^{(+)}$ & $1^{-3}$ & & $\%$ \\
\hline \multirow[t]{2}{*}{ Araraquara } & $0-20$ & 17 & 5,1 & 20 & 3,2 & 31 & 11 & 24 & 69 & 67 \\
\hline & $20-40$ & 2 & 4,3 & 14 & 2,3 & 14 & 7 & 28 & 51 & 46 \\
\hline \multirow[t]{2}{*}{ Botucatu } & $0-20$ & 4 & 4,5 & 17 & 0,7 & 6 & 5 & 26 & 38 & 32 \\
\hline & $20-40$ & 2 & 4,1 & 11 & 0,4 & 01 & 1 & 28 & 30 & 7 \\
\hline \multirow[t]{2}{*}{ Matão } & $0-20$ & 9 & 5,6 & 19 & 2,9 & 22 & 9 & 19 & 53 & 64 \\
\hline & $20-40$ & 6 & 5,2 & 17 & 1,4 & 17 & 11 & 22 & 51 & 57 \\
\hline \multirow[t]{2}{*}{ MonteAzul } & $0-20$ & 14 & 5,5 & 15 & 3,1 & 26 & 10 & 19 & 58 & 67 \\
\hline & $20-40$ & 5 & 5,2 & 10 & 1,8 & 27 & 7 & 20 & 56 & 64 \\
\hline \multirow[t]{2}{*}{ Olímpia } & $0-20$ & 22 & 4,5 & 16 & 3,0 & 13 & 4 & 33 & 53 & 35 \\
\hline & $20-40$ & 6 & 4,0 & 13 & 2,0 & 5 & 2 & 39 & 48 & 19 \\
\hline \multirow[t]{2}{*}{ Pirassununga } & $0-20$ & 45 & 5,8 & 14 & 1,2 & 26 & 8 & 15 & 50 & 71 \\
\hline & $20-40$ & 45 & 5,9 & 13 & 1,1 & 25 & 9 & 14 & 49 & 71 \\
\hline
\end{tabular}

Fonte: Cantarella et al.(1992)

As produtividades médias, em toneladas de frutas por hectare, em cada local, para cada tratamento, são apresentadas na Tabela 6 , para os experimentos fatoriais de adubação N, P e K. 
Tabela 6. Produtividade média de 4 anos de colheita (1990 a 1993) nos experimentos fatoriais de adubação N, P e K conduzidos em seis localidades no Estado de São Paulo.

\begin{tabular}{|c|c|c|c|c|c|c|c|}
\hline \multicolumn{2}{|c|}{ Tratamento } & \multirow{3}{*}{$\frac{\text { Araraquara }}{34,9}$} & \multirow{3}{*}{$\frac{\text { Botucatu }}{39,4}$} & \multirow{3}{*}{$\frac{\text { Matão }}{37,6}$} & \multirow{3}{*}{$\frac{\text { Monte Azul }}{-\mathrm{tha}^{-1}}$} & \multirow{3}{*}{$\frac{\text { Olímpia }}{26,4}$} & \multirow{3}{*}{$\frac{\text { Pirassununga }}{32,9}$} \\
\hline & & & & & & & \\
\hline & & & & & & & \\
\hline 1 & 14 & 32,4 & 43,0 & 34,3 & 20,3 & 22,9 & 40,0 \\
\hline 1 & 22 & 35,1 & 38,9 & 40,1 & 21,9 & 24,5 & 37,4 \\
\hline 1 & 23 & 35,1 & 40,4 & 35,4 & 16,8 & 20,9 & 34,9 \\
\hline 1 & 32 & 30,3 & 47,2 & 35,1 & 15,3 & 25,9 & 32,2 \\
\hline 1 & 33 & 40,8 & 38,5 & 39,1 & 16,9 & 33,3 & 34,5 \\
\hline 1 & 41 & 30,1 & 42,3 & 43,3 & 22,2 & 29,5 & 25,9 \\
\hline 1 & 44 & 38,6 & 53,1 & 41,8 & 25,7 & 28,7 & 30,9 \\
\hline 2 & 2 & 36,8 & 52,3 & 36,4 & 26,7 & 30,4 & 36,1 \\
\hline 2 & 13 & 33,8 & 46,8 & 35,8 & 25,5 & 24,7 & 36,4 \\
\hline 2 & 21 & 32,7 & 38,8 & 36,2 & 27,5 & 29,4 & 35,4 \\
\hline 2 & 24 & 36,5 & 48,5 & 36,6 & 19,6 & 22,7 & 33,1 \\
\hline 2 & 31 & 31,7 & 38,5 & 46,4 & 25,2 & 22,4 & 36,0 \\
\hline 2 & 34 & 38,1 & 51,7 & 48,7 & 30,9 & 28,0 & 34,6 \\
\hline 2 & 42 & 38,3 & 49,4 & 34,7 & 23,4 & 21,1 & 35,2 \\
\hline 2 & 43 & 39,2 & 58,1 & 44,1 & 26,6 & 27,9 & 42,4 \\
\hline 3 & 12 & 40,9 & 39,8 & 36,6 & 27,6 & 28,0 & 43,9 \\
\hline 3 & 3 & 40,2 & 51,7 & 38,5 & 29,5 & 28,9 & 28,7 \\
\hline 3 & 21 & 37,5 & 49,6 & 47,0 & 26,0 & 21,9 & 37,7 \\
\hline 3 & 24 & 41,8 & 57,5 & 42,2 & 29,4 & 26,0 & 38,5 \\
\hline 3 & 31 & 37,2 & 46,4 & 46,6 & 31,8 & 23,8 & 36,0 \\
\hline 3 & 34 & 40,4 & 49,1 & 40,1 & 27,4 & 28,0 & 43,1 \\
\hline 3 & 42 & 38,6 & 55,7 & 43,9 & 29,3 & 27,8 & 38,1 \\
\hline 3 & 43 & 37,0 & 55,8 & 39,9 & 26,0 & 22,9 & 37,6 \\
\hline 4 & 1 & 35,2 & 33,8 & 32,0 & 30,5 & 25,9 & 37,7 \\
\hline 4 & 4 & 39,3 & 48,7 & 38,3 & 27,1 & 28,4 & 34,5 \\
\hline 4 & 22 & 37,2 & 53,3 & 42,6 & 26,3 & 31,6 & 42,0 \\
\hline 4 & 23 & 39,0 & 53,9 & 45,1 & 34,2 & 27,7 & 35,5 \\
\hline 4 & 32 & 43,6 & 54,6 & 44,7 & 27,6 & 25,5 & 36,1 \\
\hline 4 & 33 & 33,3 & 52,6 & 37,8 & 28,8 & 20,8 & 42,1 \\
\hline 4 & 41 & 36,3 & 48,5 & 47,8 & 29,3 & 30,5 & 32,3 \\
\hline & 44 & 37,2 & 56,9 & 41,2 & 27,3 & 29,3 & 42,5 \\
\hline & dias & 36,8 & 48,0 & 37,6 & 25,7 & 26,4 & 36,4 \\
\hline
\end{tabular}




\subsection{Amostragem de folha e análise foliar}

A amostragem consistiu na coleta da $3^{\mathrm{a}}$ - ou $4^{\mathrm{a}}$ - folha a partir do fruto, gerada na primavera, com aproximadamente 6 meses de idade, normalmente entre fevereiro e março, em ramos com frutos de 2 a $4 \mathrm{~cm}$ de diâmetro, em número de 4 folhas por planta, uma em cada quadrante e na altura mediana da copa. Cada amostra foi composta de folhas coletadas em número mínimo de 25 plantas de acordo com o Grupo Paulista (1994) e acondicionadas em saco de papel devidamente identificado.

Essas amostras foram enviadas para o laboratório da SFSNP/IAC para a análise química dos teores totais dos macronutrientes seguindo os procedimentos de Bataglia et al.(1983). As amostras analisadas a partir de 1993 seguiram os novos procedimentos para determinação dos teores de fósforo, potássio, cálcio e magnésio através da técnica conhecida como ICP ou espectrofotometria de plasma induzido, contudo os extratos continuam os mesmos de Bataglia et al.(1983).

\subsection{Pomares comerciais}

A rede de ensaios de adubação $\mathrm{N}, \mathrm{P}$ e $\mathrm{K}$ em citros foi instalada em propriedades particulares tomadas como modelo na área citrícola. Dentre os pomares comerciais, os da empresa Cambuhy Empreendimentos Agropecuários foi selecionado por apresentar um banco de dados histórico e de alta representatividade no que diz respeito à combinação copa $\mathrm{x}$ porta-enxerto. A empresa é altamente competitiva em várias culturas como citros, seringueira e café. Para tanto, conta com um corpo técnicoadministrativo de alto nível que visa a otimização dos recursos para obtenção de resultados financeiros.

A área citrícola está dividida em talhões com plantas de mesmas características (combinação copa x porta-enxerto, idade), possibilitando um tratamento individualizado dessas áreas. Essa característica aliada a confiabilidade de informações sobre a fertilidade do solo, do estado nutricional das plantas e da produtividade, permitiu a utilização desses dados como primeira inferência sobre uma população de referência para os citros do Estado de São Paulo. 


\subsection{Cálculo dos índices DRIS}

O trabalho foi desenvolvido na Seção de Fertilidade do Solo e Nutrição de Plantas do Instituto Agronômico-Campinas, nos equipamentos de informática e "softwares" disponíveis desenvolvidos em dois Projetos de Iniciação Científica* e do programa estatístico para o delineamento experimental fatorial fracionado (1/2) $4^{3}$ desenvolvido na Seção de Técnica Experimental e Cálculo do IAC.

Os Índices-DRIS para os macronutrientes foram calculados pelos procedimentos propostos por Beaufils (1973), Beaufils modificado por Elwali \& Gascho (1984) e Jones (1981). Então, obteve-se o índice DRIS IY: para o nutriente Y da seguinte forma:

$$
\frac{\sum_{i=1}^{m} f\left(Y / X_{i}\right)-\sum_{j=1}^{n} f\left(X_{j} / Y\right)}{m+n}
$$

Beaufils (1973)

$$
\mathrm{f}(\mathrm{Y} / \mathrm{X})= \begin{cases}\left(1-\frac{\mathrm{Y} / \mathrm{X}(\mathrm{p})}{\mathrm{Y} / \mathrm{X}(\mathrm{a})}\right) \cdot \frac{100 . k}{\mathrm{CV} \%}, & \text { para } \mathrm{Y} / \mathrm{X}(\mathrm{a})<\mathrm{Y} / \mathrm{X}(\mathrm{p}) \\ 0, & \text { para } \mathrm{Y} / \mathrm{X}(\mathrm{a})=\mathrm{Y} / \mathrm{X}(\mathrm{p}) \\ \left(\frac{\mathrm{Y} / \mathrm{X}(\mathrm{a})}{\mathrm{Y} / \mathrm{X}(\mathrm{p})}-1\right) \cdot \frac{100 . k}{\mathrm{CV} \%}, & \text { para } \mathrm{Y} / \mathrm{X}(\mathrm{a})>\mathrm{Y} / \mathrm{X}(\mathrm{p})\end{cases}
$$

Beaufils Modificado, Elwali \& Gascho (1984)

$$
\mathrm{f}(\mathrm{Y} / \mathrm{X})= \begin{cases}\left(1-\frac{\mathrm{Y} / \mathrm{X}(\mathrm{p})}{\mathrm{Y} / \mathrm{X}(\mathrm{a})}\right) \cdot \frac{100 \cdot k}{\mathrm{CV} \%}, & \text { para } \mathrm{Y} / \mathrm{X}(\mathrm{a})<\mathrm{Y} / \mathrm{X}(\mathrm{p}) \\ 0, & \text { para } \mathrm{Y} / \mathrm{X}(\mathrm{p})-s \leq \mathrm{Y} / \mathrm{X}(\mathrm{a}) \leq \mathrm{Y} / \mathrm{X}(\mathrm{p})+s \\ \left(\frac{\mathrm{Y} / \mathrm{X}(\mathrm{a})}{\mathrm{Y} / \mathrm{X}(\mathrm{p})}-1\right) \cdot \frac{100 \cdot k}{\mathrm{CV} \%}, & \text { para } \mathrm{Y} / \mathrm{X}(\mathrm{a})>\mathrm{Y} / \mathrm{X}(\mathrm{p})\end{cases}
$$

Jones (1981):

$\mathrm{f}(\mathrm{Y} / \mathrm{X})=\quad(\mathrm{Y} / \mathrm{X}(\mathrm{a})-\mathrm{Y} / \mathrm{X}(\mathrm{p})) k / s$

\footnotetext{
- Relatórios Científicos apresentados a FAPESP pelo bolsista de Iniciação Científica Wagner Rodrigues dos Santos sob orientação do prof.Dr Ondino Cleante Bataglia referentes aos projetos "Desenvolvimento de software para o Sistema Integrado de Diagnose e Recomendação - DRIS, em café, citros e seringueira" (1989) e "Avaliaçăo do DRIS como critério alternativo de interpretação de análise foliar em citros" (1992).
} 
Onde: $\mathrm{Y} / \mathrm{X}(\mathrm{a})$ - relação na amostra;

$\mathrm{Y} / \mathrm{X}(\mathrm{p})$ - relação na população não-anormal;

CV\% - coeficiente de variação para a relação, \%;

$s \quad$ - desvio padrão da relação;

$k \quad$ - constante de sensibilidade, valor adotado neste trabalho foi igual a 20;

O balanço nutricional médio (B.N.) foi calculado a partir dos valores absolutos dos índices gerados para a amostra, dividido pelo número de nutrientes que participam do seu cálculo. Na realidade o B.N. assim calculado é um índice-DRIS médio, o qual permite comparações entre B.N.'s provenientes de diferentes conjuntos de nutrientes. Assim temos, por exemplo, para os índices gerados para o conjunto contendo apenas os macronutrientes:

$$
\text { B.N. }=\left(\left|I_{N}\right|+\left|I_{P}\right|+\left|I_{K}\right|+\left|I_{C a}\right|+\left|I_{M g}\right|+\left|I_{S}\right|\right) / 6
$$

\subsection{Formas de análise dos resultados}

Foram levantadas, nos arquivos da SFSNP-IAC, as análises químicas das folhas de amostras dos experimentos do projeto, no total de 912 amostras, e ainda amostras de 4 anos provenientes de pomares produtivos da Cambuhy Empreendimentos Agropecuários, Matão. Em seguida, foram construídos arquivos no formato "DBF" para permitir a estruturação de uma base de dados citrícolas, inicialmente com os teores foliares dos nutrientes minerais (macro e micronutrientes) e produtividade, com 0 auxílio do software "dBASE III PLUS". Os dados de análise foliar (macronutrientes: $\mathrm{N}$, $\mathrm{P}, \mathrm{K}, \mathrm{Ca}, \mathrm{Mg}$ e S), de solo (P, M.O., $\mathrm{pH}, \mathrm{K}, \mathrm{Ca}, \mathrm{Mg}$ e $\mathrm{H}+\mathrm{Al}$ ) e produtividade (caixas de frutos/planta e tha) foram processados com o auxílio do programa para análise estatística de experimento em esquema fatorial incompleto (1/2) $4^{3}$. Foram estimados os demais tratamentos para se completar o esquema fatorial $4^{3}$ e minimizar a influência da variação do acaso (variações climáticas e solo entre outras); e então, organizados para obtenção de padrões de referência para o cálculo dos Índices-DRIS das amostras nos diferentes ensaios.

A partir desses dados, corrigidos estatisticamente, foram separadas amostras com produtividade relativa maior ou igual a $90 \%$, para cada local, sendo assim definida a população de alta produtividade. A população de baixa produtividade foi definida com as amostras com produtividade relativa abaixo de $90 \%$. Foram calculados os parâmetros estatísticos como a média, variância, desvio padrão e coeficiente de 
variação para cada relação entre os nutrientes, como nos procedimentos usados por Letzsch (1985). A necessidade do tratamento individualizado dos teores médios em cada local, foi devido às diferenças significativas evidenciadas pela análise da variância entre os locais.

Através da população de referência e dados de teores foliares dos macronutrientes foram calculados os índices - DRIS, segundo os métodos propostos por Beaufils (1973) e Jones (1981), para a definição do procedimento padrão de cálculo. A avaliação do melhor procedimento de cálculo foi baseada nos coeficientes de correlação entre o Índice DRIS médio (B.N., também denominado, Balanço Nutricional) e a produtividade.

Uma outra linha para a definição da população de referência foi a seleção de amostras em condições reais de campo, em pomares comerciais. As amostras da rede de ensaio de adubação N, P e K, então, foram utilizadas para a verificação e interpretação da diagnose feita pelos métodos em avaliação.

A verificação de interações entre nutrientes, entre nutrientes e produtividade, e resposta da produtividade à adubação, foi realizada estatisticamente, através de análise da variância e correlações múltiplas.

A diagnose ou avaliação do teor foliar foi feita pelo Critério de Faixas de Teores (Tabela 7), adotado pelo Grupo Paulista de Adubação e Calagem para Citros (1994) e pelo DRIS através dos três procedimentos de cálculo descritos.

A consistência da diagnose foi testada através da comparação entre os dois critérios pela recomendação indicada e o resultado obtido em termos de produtividade.

A eficiência dos métodos seguiu o protocolo proposto por Beverly \& Hallmark (1992), através de três índices quantitativos: a exatidão da diagnose, a razão entre as diagnoses positivas $\left(\mathrm{V}^{+}: \mathrm{F}^{+}\right)$e a resposta líquida acumulada.

Para cada método e local experimental foram analisados todos os tratamentos passíveis de uma verificação do fornecimento do próximo nível do nutriente via fertilizante, quanto a diagnose e a resposta da produtividade. $O$ tratamento com algum nível 3 não fez parte da avaliação pois não havia tratamento com dose maior para este nutriente, o que inviabilizou a determinação da coerência da diagnose com a resposta da produtividade.

A partir do tratamento testemunha no qual foi feita a diagnose para cada nutriente pelos diferentes métodos, pode-se classificar a diagnose em função da 
resposta em: $\mathrm{V}^{+}$(diagnose indicou a necessidade de aplicação do fertilizante contendo o nutriente, e quando este foi aplicado a produtividade apresentou uma resposta positiva ${ }^{1}$ ), $\mathrm{V}^{-}$(diagnose recomendou que o fertilizante não fosse aplicado desnecessariamente), $\mathrm{F}^{+}$ (apesar da diagnose recomendar a aplicação do fertilizante a resposta da produtividade foi desfavorável) e $F^{-}$(o método diagnóstico não foi eficiente para recomendar a aplicação, acarretando uma perda de oportunidade de melhorar a produtividade); e calcular o incremento ou redução da produtividade: a resposta. $\mathrm{O}$ mesmo procedimento foi usado com o nível seguinte do nitrogênio, mantendo-se os níveis de fósforo e de potássio inalterados, e assim sucessivamente para todos os 48 tratamentos. Foram totalizados o número de ocorrência de diagnoses $\mathrm{V}^{+}, \mathrm{V}^{-}, \mathrm{F}^{+}$e F- bem como, as respostas de produtividade líquida para as aplicações de N, P e K. Com esses valores foi possível calcular a exatidão que é o total de diagnoses corretas $\left(\mathrm{V}^{+}+\mathrm{V}^{-}\right)$; a razão das diagnoses positivas $\left(\mathrm{V}^{+}: \mathrm{F}^{+}\right)$; e o total da resposta da produtividade líquida $(\mathrm{d}(\mathrm{Y}))$ para N, P e K.

Os valores determinados por estes procedimentos foram convertidos a valores percentuais do total de tratamentos avaliados (48 tratamentos) e tabelados a fim de permitir a comparação entre os métodos diagnósticos.

\footnotetext{
1 Beverly \& Hallmark (1992) recomendam que, para a avaliação em que não se tenha quantificada a variação da produtividade, seja considerada resposta positiva ou negativa sempre que a diferença de produtividade entre dois tratamentos for superior a $10 \%$ da produtividade do tratamento em teste. Contudo, neste trabalho foi feita uma simplificação e resposta positiva é quando o d(Y) for maior que zero e resposta desfavorável quando $d(Y)$ for menor ou igual a zero.
} 
Tabela 7. Faixas de teores dos macronutrientes nas folhas de citros, geradas na primavera, com seis meses de idade, de ramos frutíferos

\begin{tabular}{cccc}
\hline & \multicolumn{3}{c}{ Classificação do teor foliar } \\
\cline { 2 - 4 } Nutriente & Baixo & Adequado & Excessivo \\
\cline { 2 - 4 } & $<22$ & $\mathrm{~g} \mathrm{~kg}^{-1}$ & $>27$ \\
$\mathrm{~N}$ & 22 a 27 & $>1,6$ \\
$\mathrm{P}$ & 1,1 a 1,6 & $>15$ \\
$\mathrm{~K}$ & $<10$ & 10 a 15 & $>45$ \\
$\mathrm{Ca}$ & $<35$ & 35 a 45 & $>4,0$ \\
$\mathrm{Mg}$ & $<2,5$ & 2,5 a 4,0 & $>3,0$ \\
$\mathrm{~S}$ & $<2,0$ & 2,0 a 3,0 & \\
\hline
\end{tabular}

Fonte: Grupo Paulista de Adubação e Calagem para Citros (1994). 


\section{RESULTADOS E DISCUSSÃO}

\subsection{Definição da população de referência}

Inicialmente, foi procurada uma população de referência tomando-se como base os teores foliares dos melhores tratamentos de cada local da rede de ensaios de adubação N, P e K. Contudo, estes teores eram resultados de adubações segundo um plano experimental, ou seja, com doses de subsistência da planta até doses de "luxo". Essa condição não representa a condição dos pomares cítricos do Estado de São Paulo, além do que a reduzida diversidade de combinações (copa x porta-enxerto, densidade de plantio, entre outras) proporcionou o realce de duas produtividades distintas de maior ocorrência, observada através da curva de distribuição de frequêencia das classes de produtividade (Figura 1). Esse fato sugere o efeito de algum fator não controlado, que apesar da tentativa de discretização da população por local do ensaio, não foi suficiente para viabilizar o uso generalizado do DRIS.

Neste trabalho estava prevista a caracterização da população de referência para citros, como primeira inferência no âmbito paulista, dada a representatividade dos dados quanto às regiões produtoras e combinações copa/portaenxerto. No entanto, como já havia sido determinada a influência de fatores locais sobre o desempenho produtivo da cultura e teores foliares, optou-se pela confecção de pelo menos três linhas de referência, uma geral com todos os locais e outra para cada local (dados da rede de ensaios de adubação $\mathrm{N}, \mathrm{P}$ e $\mathrm{K}$ ), e a terceira com dados de uma propriedade modelo. 
Procurando-se minimizar a predominância de amostras de um determinado local com desempenho produtivo maior que os demais locais e, consequentemente comprometendo a diagnose, efetuou-se a seleção de amostras com produtividade relativa igual ou superior a $\mathbf{9 0 \%}$ para compor a população de alta produtividade e a de baixa produtividade com amostras de produtividade relativa inferior a $90 \%$.

Alguns parâmetros estatísticos do grupo de experimentos para os teores médios foliares e produtividade estão apresentados na Tabela 8, na qual observase que para todas as variáveis há diferenças de teores médios foliares e produtividade entre os ensaios, causadas possivelmente, pelas diferentes combinações copa/portaenxerto de citros ou mesmo pelas condições locais de clima e solo.

A existência de efeito do "local" (copa/porta-enxerto, condições de solo e clima) sobre as variáveis estudadas, foi primeiramente observado, através de parâmetros estatísticos (média e desvio padrão) na Tabela 8. Então, foram realizadas as comparações das médias dos teores foliares e da produtividade entre cada local através do Teste $t$, sob a hipótese de não haver diferença significativa entre as médias das variáveis $\left(\mathrm{H}_{0}: \mu_{1}=\mu_{2}\right)$. Os valores limites para $t$ aos níveis de $5 \%$ e $1 \%$ foram 1,980 e 2,617 , respectivamente.

Os valores de $t$ da comparação entre cada local para cada variável são apresentados na Tabela 9. O efeito do local foi menor sobre os teores foliares médios do fósforo e do enxôfre, no entanto, a diferença entre locais dos teores foliares médios dos demais macronutrientes e da produtividade média foi significativa, o que reforça $o$ conceito da influência da copa e do porta-enxerto, bem como de fatores edafoclimáticos, no teor foliar e na produção.

$\mathrm{Na}$ Tabela 10 são apresentados os teores foliares médios das populações A (produtividade relativa acima de $90 \%$ ) e B (produtividade relativa abaixo de 90\%), para cada um dos seis locais, para todos os seis locais e para os cinco locais com laranjeiras. A exclusão dos dados do Limoeiro Siciliano, foi por este apresentar o teor médio de nitrogênio, normalmente, muito inferior ao dos demais citros em estudo. Para cada local foram calculados os parâmetros estatísticos, média, variância, desvio 
padrão e coeficiente de variação, para os nutrientes e relações entre nutrientes. Estas informações estão nos Apêndices 1 a 6.

Em razão da dificuldade de se caracterizar uma população única através da rede de ensaios, como já foi discutida anteriormente, procurou-se então estabelecer uma população de referência usando-se os dados de quatro anos (1991 a 1994) provenientes de pomares comerciais da empresa Cambuhy Empreendimentos Agropecuários Ltda., que passou a ser considerada como local modelo. Essas amostras também são representativas das combinações copa/porta-enxerto normalmente encontradas nos pomares citrícolas, estando caracterizadas na Tabela 11. Conhecida a distribuição de freqüência das faixas de produtividade (Figura 1), que se aproxima da distribuição normal, procedeu-se a classificação das amostras em três classes de produtividade:

- 1: amostras com produtividade inferior a $\mu-2 / 3 s$

- 2: amostras com produtividade na faixa entre $\mu-2 / 3$ s e $\mu+2 / 3$ s

- 3: amostras com produtividade superior a $\mu+2 / 3 s$

A partir dessa classificação pode-se compor as populações A e B (populações com produtividades médias satisfatória, próxima a $30 \mathrm{tha}^{-1}$ e indesejável, $17,0 \mathrm{tha}^{-1}$ respectivamente), considerando as amostras da classe 1 como pertencentes a população B ( $\mu$ - $2 / 3 s)$, a qual possui comportamento distinto em relação a população $\mathrm{A}$ de referência, obtida no intervalo entre $\mu-2 / 3 s e \mu+2 / 3 s$. Esta população de referência representa $50 \%$ do conjunto inicial de dados. O estabelecimento de populações com produtividades distintas é requisito para estudos com DRIS. As amostras pertencentes a classe 3 não foram consideradas pois apresentaram produtividades muito altas e o interesse desse trabalho é a de detectar possíveis desequilíbrios nutricionais existentes nos pomares de baixa produtividade, para numa primeira fase elevar essa produtividade a níveis aceitáveis economicamente de cerca de $30 \mathrm{t} / \mathrm{ha}$. Esta produtividade média é muito superior a produtividade média da citricultura, considerada ao redor de 2 caixas/planta ou $17 \mathrm{t} / \mathrm{ha}$ no espaçamento tradicional, $(8,0 \times 6,0) \mathrm{m}$. 
Tabela 8. Parâmetros estatísticos dos teores foliares e produtividade de cada local experimental, referentes ao período de 1989 a 1992.

\begin{tabular}{|c|c|c|c|c|c|c|c|}
\hline & $\mathrm{N}$ & $\mathbf{P}$ & $\mathrm{K}$ & $\mathrm{Ca}$ & $\mathrm{Mg}$ & $\mathbf{S}$ & Produtividade \\
\hline & & & $-\mathrm{g}$ & & & & $\mathrm{tha}^{-1}$ \\
\hline \multicolumn{8}{|c|}{ Araraquara } \\
\hline Mínimo & 23,3 & 1,1 & 11,6 & 26,8 & 1,8 & 1,8 & 30,1 \\
\hline Máximo & 28,0 & 1,6 & 16,8 & 35,4 & 3,1 & 2,9 & 43,6 \\
\hline Amplitude & 4,7 & 0,5 & 5,2 & 8,6 & 1,3 & 1,0 & 13,5 \\
\hline Média & 26,1 & 1,3 & 14,6 & 32,3 & 2,4 & 2,5 & 36,8 \\
\hline $\mathbf{s}$ & 1,2 & 0,1 & 1,3 & 2,0 & 0,3 & 0,2 & 3,3 \\
\hline CV\% & 4,0 & 9,0 & 9,0 & 6,0 & 14,0 & 9,0 & 9,0 \\
\hline \multicolumn{8}{|l|}{ Botucatu } \\
\hline Mínimo & 17,2 & 1,2 & 3,2 & 26,7 & 3,6 & 1,6 & 33,8 \\
\hline Máximo & 23,6 & 1,6 & 15,1 & 54,5 & 7,4 & 2,8 & 58,1 \\
\hline Amplitude & 6,4 & 0,4 & 11,9 & 27,8 & 3,8 & 1,2 & 24,3 \\
\hline Média & 20,4 & 1,3 & 7,6 & 38,8 & 5,2 & 2,2 & 47,9 \\
\hline s & 1,8 & 0,1 & 3,7 & 6,7 & 1,0 & 0,3 & 6,7 \\
\hline CV\% & 8,0 & 9,0 & 48,0 & 17,0 & 19,0 & 16,0 & 14,1 \\
\hline \multicolumn{8}{|l|}{ Matão } \\
\hline Mínimo & 24,0 & 1,0 & 10,8 & 34,0 & 3,5 & 1,7 & 32,0 \\
\hline Máximo & 30,1 & 1,4 & 19,9 & 42,4 & 4,9 & 3,1 & 48,7 \\
\hline Amplitude & 6,1 & 0,4 & 9,1 & 8,4 & 1,4 & 1,3 & 16,7 \\
\hline Média & 27,2 & 1,2 & 16,1 & 37,9 & 4,0 & 2,6 & 40,3 \\
\hline $\mathbf{s}$ & 1,7 & 0,0 & 2,4 & 2,3 & 0,3 & 0,3 & 4,5 \\
\hline CV\% & 6,0 & 6,0 & 15,0 & 6,0 & 8,0 & 11,0 & 11,2 \\
\hline \multicolumn{8}{|l|}{ Monte Azul } \\
\hline Mínimo & 21,4 & 1,0 & 9,8 & 36,5 & 2,0 & 2,0 & 15,3 \\
\hline Máximo & 27,9 & 1,7 & 16,0 & 47,5 & 3,8 & 2,9 & 34,2 \\
\hline Amplitude & 6,5 & 0,7 & 6,2 & 11,0 & 1,8 & 0,9 & 18,9 \\
\hline Média & 24,0 & 1,3 & 12,6 & 42,1 & 2,7 & 2,4 & 25,7 \\
\hline s & 1,2 & 0,1 & 1,8 & 3,0 & 0,4 & 0,2 & 4,5 \\
\hline CV\% & 5,0 & 12,0 & 14,0 & 7,0 & 14,0 & 9,0 & 17,7 \\
\hline \multicolumn{8}{|l|}{ Olimpia } \\
\hline Mínimo & 25,9 & 1,0 & 9,1 & 25,8 & 3,4 & 2,1 & 20,8 \\
\hline Máximo & 31,8 & 1,5 & 14,4 & 35,2 & 4,9 & 3,2 & 33,3 \\
\hline Amplitude & 5,9 & 0,4 & 5,3 & 9,4 & 1,5 & 1,0 & 12,5 \\
\hline Média & 28,8 & 1,3 & 11,7 & 31,8 & 4,4 & 2,9 & 26,4 \\
\hline$s$ & 1,3 & 0,0 & 1,2 & 2,0 & 0,3 & 0,2 & 3,3 \\
\hline CV\% & 4,0 & 6,0 & 10,0 & 6,0 & 6,0 & 7,0 & 12,6 \\
\hline \multicolumn{8}{|c|}{ Pirassununga } \\
\hline Mínimo & 22,0 & 1,0 & 7,3 & 39,4 & 3,5 & 2,0 & 25,9 \\
\hline Máximo & 27,8 & 1,4 & 15,0 & 50,2 & 5,5 & 2,4 & 43,9 \\
\hline Amplitude & 5,8 & 0,3 & 7,7 & 10,8 & 2,0 & 0,3 & 18,0 \\
\hline Média & 23,9 & 1,2 & 10,4 & 44,2 & 4,1 & 2,2 & 36,3 \\
\hline s & 1,2 & 0,1 & 1,7 & 2,6 & 0,4 & 0,1 & 4,1 \\
\hline CV\% & 5,0 & 8,0 & 17,0 & 6,0 & 10,0 & 4,0 & 11,5 \\
\hline
\end{tabular}


Tabela 9. Comparação das médias dos teores foliares e das produtividades entre cada local experimental através do Teste $t$.

\begin{tabular}{|c|c|c|c|c|c|c|}
\hline Variáveis & Locais & Araraquara & Botucatu & Matão & Monte Azul & Olímpia \\
\hline Nitrogênio & $\begin{array}{l}\text { Botucatu } \\
\text { Matão } \\
\text { Monte Azul } \\
\text { Olímpia } \\
\text { Pirassununga }\end{array}$ & $\begin{array}{l}\mathbf{3 , 7 2 6 * *} \\
0,748 \\
1,750 \\
\mathbf{2 , 1 5 8 *} \\
1,833\end{array}$ & 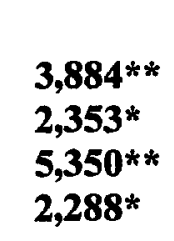 & $\begin{array}{l}\mathbf{2 , 1 7 5 ^ { * }} \\
1,057 \\
\mathbf{2 , 2 4 3 ^ { * }}\end{array}$ & $\begin{array}{l}\mathbf{3 , 8 3 7} 7^{* *} \\
0,083\end{array}$ & $3,917^{* *}$ \\
\hline Fósforo & $\begin{array}{l}\text { Botucatu } \\
\text { Matão } \\
\text { Monte Azul } \\
\text { Olímpia } \\
\text { Pirassununga }\end{array}$ & $\begin{array}{l}0,000 \\
0,128 \\
0,000 \\
0,000 \\
0,128\end{array}$ & $\begin{array}{l}1,000 \\
0,000 \\
0,000 \\
1,000\end{array}$ & $\begin{array}{l}1,000 \\
1,000 \\
0,000\end{array}$ & $\begin{array}{l}0,000 \\
1,000\end{array}$ & 1,000 \\
\hline Potássio & $\begin{array}{l}\text { Botucatu } \\
\text { Matão } \\
\text { Monte Azul } \\
\text { Olímpia } \\
\text { Pirassununga }\end{array}$ & 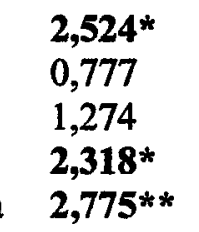 & $\begin{array}{l}2,726^{* *} \\
1,719 \\
1,491 \\
0,972\end{array}$ & $\begin{array}{l}1,650 \\
2,319^{*} \\
2,741^{* *}\end{array}$ & $\begin{array}{l}0,588 \\
1,257\end{array}$ & 0,884 \\
\hline Cálcio & $\begin{array}{l}\text { Botucatu } \\
\text { Matão } \\
\text { Monte Azul } \\
\text { Olímpia } \\
\text { Pirassununga }\end{array}$ & $\begin{array}{l}1,315 \\
\mathbf{2 , 5 9 8 *} \\
\mathbf{3 , 8 4 4}^{* *} \\
0,250 \\
\mathbf{5 , 1 3 0 * *}\end{array}$ & $\begin{array}{l}0,180 \\
0,636 \\
1,416 \\
1,063\end{array}$ & $\begin{array}{l}1,571 \\
\mathbf{2 , 8 3 0 * *} \\
\mathbf{2 , 5 6 7 *}\end{array}$ & $\begin{array}{l}4,040^{* *} \\
0,748\end{array}$ & $5,346 * *$ \\
\hline Magnésio & $\begin{array}{l}\text { Botucatu } \\
\text { Matão } \\
\text { Monte Azul } \\
\text { Olímpia } \\
\text { Pirassununga }\end{array}$ & $\begin{array}{l}\mathbf{3 , 7 9 3} 3^{* *} \\
\mathbf{5 , 3 3 3}^{* *} \\
0,849 \\
\mathbf{6 , 6 6 7 ^ { * * }} \\
\mathbf{4 , 8 0 8 ^ { * * }}\end{array}$ & $\begin{array}{l}1,625 \\
3,283 \text { ** } \\
1,084 \\
1,444\end{array}$ & $\begin{array}{l}3,677^{* *} \\
1,333 \\
0,283\end{array}$ & $\begin{array}{l}4,808^{* *} \\
\mathbf{3 , 5 0 0 ^ { * * }}\end{array}$ & 0,849 \\
\hline Enxôfre & $\begin{array}{l}\text { Botucatu } \\
\text { Matão } \\
\text { Monte Azul } \\
\text { Olímpia } \\
\text { Pirassununga }\end{array}$ & $\begin{array}{l}1,177 \\
0,392 \\
0,500 \\
\mathbf{2 , 0 0 0 *} \\
1,897\end{array}$ & $\begin{array}{l}1,333 \\
0,784 \\
2,746^{* *} \\
0,000\end{array}$ & $\begin{array}{l}0,784 \\
1,177 \\
1,789\end{array}$ & $\begin{array}{l}2,500^{*} \\
1,265\end{array}$ & $4,427^{* *}$ \\
\hline Produtividac & $\begin{array}{l}\text { de Botuc } \\
\text { Matão } \\
\text { Monte Azul } \\
\text { Olímpia } \\
\text { Pirassununga }\end{array}$ & $\begin{array}{l}\text { ceatu } \\
0,887 \\
\mathbf{2 , 8 1 3 ^ { * * }} \\
\mathbf{3 , 1 5 2 * *} \\
0,134\end{array}$ & 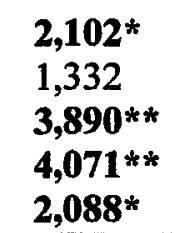 & $\begin{array}{l}\mathbf{3 , 2 4 4} \\
\mathbf{3 , 5 2 3}^{* *} \\
0,929\end{array}$ & $\begin{array}{l}0,177 \\
2,462^{*}\end{array}$ & $2,660^{\text {** }}$ \\
\hline
\end{tabular}

*,** diferenças entre médias significativas aos níveis de 5 e $1 \%$ de probabilidade, respectivamente. 
Tabela 10. Teores foliares médios dos macronutrientes para oito conjuntos de amostras tomados para compor as populações de referência de alta $(\mathrm{A})$ produtividade relativa igual ou superior a $90 \%$ e baixa (B) produtividade relativa menor que $90 \%$.

\begin{tabular}{|c|c|c|c|c|c|c|c|c|c|c|c|c|}
\hline \multirow{2}{*}{ População } & \multicolumn{2}{|c|}{$\mathrm{N}$} & \multicolumn{2}{|c|}{$P$} & \multicolumn{2}{|c|}{$\mathrm{K}$} & \multicolumn{2}{|c|}{$\mathrm{Ca}$} & \multicolumn{2}{|c|}{$\mathrm{Mg}$} & \multicolumn{2}{|c|}{$S$} \\
\hline & A & B & $\mathrm{A}$ & B & A & B & A & B & $\mathrm{A}$ & B & $\mathrm{A}$ & B \\
\hline Araraquara & 26,4 & 25,7 & 1,3 & 1,4 & 14,8 & 14,4 & 32,6 & 32,1 & 2,4 & 2,4 & 2,5 & 2,7 \\
\hline Botucatu & 21,2 & 19,7 & 1,4 & 1,3 & 8,1 & 7,0 & 37,8 & 40,0 & 5,1 & 5,5 & 2,1 & 2,3 \\
\hline Matão & 27,8 & 26,6 & 1,3 & 1,3 & 16,1 & 16,3 & 38,3 & 37,6 & 4,1 & 4,1 & 2,6 & 2,6 \\
\hline Monte Azul & 24,4 & 23,5 & 1,3 & 1,5 & 12,4 & 13,0 & 42,4 & 41,8 & 2,8 & 2,7 & 2,4 & 2,6 \\
\hline Olímpia & 29,1 & 28,6 & 1,3 & 1,4 & 11,5 & 11,9 & 30,9 & 32,6 & 4,3 & 4,6 & 2,9 & 3,0 \\
\hline Pirassununga & 24,0 & 23,8 & 1,2 & 1,3 & 9,9 & 11,0 & 44,5 & 43,8 & 4,2 & 3,9 & 2,3 & 2,3 \\
\hline Geral I & 26,3 & 24,4 & 1,3 & 1,3 & 12,9 & 11,7 & 36,4 & 38,9 & 3,6 & 4,0 & 2,5 & 2,5 \\
\hline Geral II & 26,6 & 25,6 & 1,3 & 1,3 & 13,3 & 13,0 & 36,3 & 38,8 & 3,5 & 3,6 & 2,6 & 2,6 \\
\hline
\end{tabular}

Geral I e II - população geral com e sem amostras de Lim.Siciliano, respectivamente. 


\begin{tabular}{clr}
\hline Classe & \multicolumn{2}{c}{$\begin{array}{c}\text { Faixas de } \\
\text { produtividade }\end{array}$} \\
\hline \multicolumn{3}{c}{ t ha $^{-1}$} \\
A & \multicolumn{2}{c}{ menor que 15} \\
B & 15 & 20 \\
C & 20 & 25 \\
D & 25 & 30 \\
E & 30 & 35 \\
F & 35 & 40 \\
G & 40 & 45 \\
H & 45 & 50 \\
I & 50 & 55 \\
J & 55 & 60 \\
K & 60 & 65 \\
L & maior que 65 \\
\hline
\end{tabular}

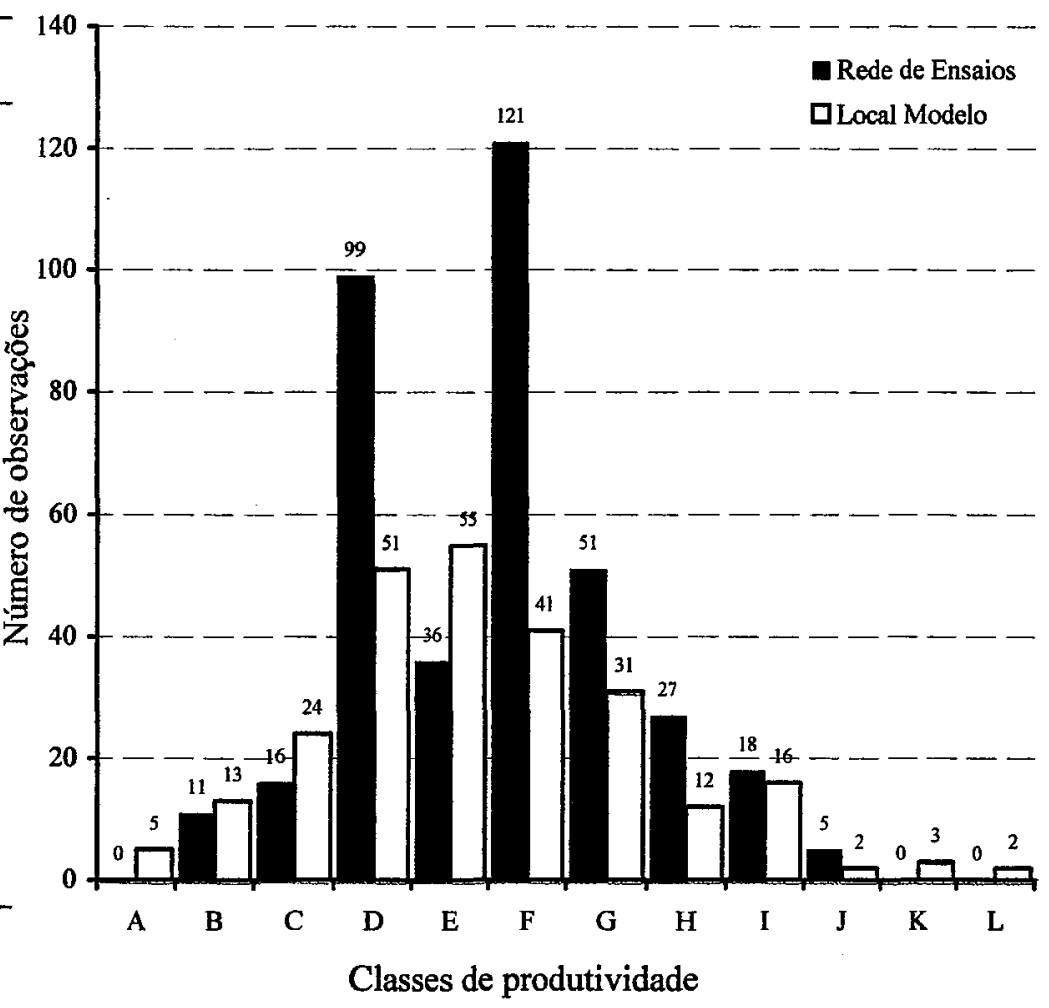

Figura 1. Distribuição de frequiências das produtividades para dois conjuntos de dados, produtividades dos locais da rede de ensaios de adubação $\mathrm{N}, \mathrm{P}$ e K, e amostras de um pomar modelo.

A escolha das relações entre nutrientes para o cálculo dos índices DRIS foi feita através da razão entre variâncias das populações de produtividade média e baixa (Teste F, segundo Steel \& Torrie, 1960), sendo que a relação entre nutrientes que melhor realça o parâmetro de interesse, a produtividade, é aquela que apresenta a maior razão entre as variâncias. Os parâmetros estatísticos da população bem como a razão entre variâncias estão no Apêndice 8 (população com amostras no intervalo compreendido entre $\mu \pm 2 / 3 s$, para verificar a influência da faixa de seleção que compõe a população sobre a diagnose).

A principal diferença observada entre as produtividades médias foi entre as laranjeiras Pêra (menor produtividade) e Hamlin e Valência (de maior). 
Para esse conjunto de dados não foram observadas diferenças significativas em termos de produtividade ( $t / h a$ ), para os efeitos de espaçamento, para a mesma combinação copa $\mathrm{x}$ porta-enxerto, e nem a combinação copa $\mathrm{x}$ porta-enxerto para o mesmo espaçamento, o que não acontecia com a população anterior, baseada apenas nos ensaios (Tabela 12).

Ainda, com relação ao conjunto de dados que foi considerado como referência para as condições da citricultura paulista, pode-se observar que a densidade de plantio e os porta-enxertos foram fatores que não influenciaram de forma significativa os teores médios dos macronutrientes. Contudo, a laranjeira Hamlin apresentou teor de nitrogênio e enxofre maior que a laranjeira Pêra quando ambas estão sobre limoeiro Cravo. Isto sugere um comportamento diferenciado na absorção e metabolização desses dois macronutrientes. No entanto, a resposta em termos de produtividade também acompanhou os teores foliares, ou seja, níveis maiores de nutrientes foram associados a maiores produtividades. Também foi observado que o limoeiro Siciliano tem comportamento nutricional diferenciado das demais variedades, o que requer um cuidado especial na interpretação da diagnose, quando esta foi feita baseada em Faixas de Teores Adequados.

Tabela 11. Características dos talhões do conjunto de amostras de folhas e de produtividade, utilizadas para o estabelecimento da população de referência para o uso do DRIS.

\begin{tabular}{llccc}
\hline Copa & $\begin{array}{l}\text { Porta- } \\
\text { Enxerto }\end{array}$ & $\begin{array}{l}\text { Espaçamento } \\
\text { (E.L.xE.P.) }\end{array}$ & $\begin{array}{c}\text { No. } \\
\text { Amostras }\end{array}$ & Distribuição \\
\hline Valência & & $\mathrm{m}$ & & $\%$ \\
Hamlin & Cravo & $\mathbf{8 , 0 \times 6 , 0}$ & 60 & 23,5 \\
Hamlin & Cravo & $8,0 \times 5,0$ & 2 & 0,8 \\
Natal & Cravo & $\mathbf{8 , 0 \times 6 , 0}$ & 8 & 3,1 \\
Pêra & Cravo & $8,0 \times 6,0$ & 66 & 25,9 \\
Pêra & Cravo & $8,0 \times 6,0$ & 74 & 29,0 \\
Valência & Cravo & $8,0 \times 4,5$ & 19 & 7,5 \\
Natal & Volkameriana & $8,0 \times 6,0$ & 18 & 7,1 \\
\hline
\end{tabular}


Tabela 12. Valores médios dos teores foliares e produtividade do grupo de dados (combinações copa $\mathrm{x}$ porta-enxerto $\mathrm{x}$ espaçamento), acompanhados do Teste de Tukey ao nível de $5 \%$ de significância, do qual se extraiu a população de referência, não-anormal, para o DRIS.

\begin{tabular}{|c|c|c|c|c|c|c|}
\hline $\begin{array}{c}\mathbf{N} \\
\text { Trat.Médias }\end{array}$ & $\begin{array}{c}\mathrm{P} \\
\text { Trat.Médias }\end{array}$ & $\begin{array}{c}\mathrm{K} \\
\text { Trat.Médias }\end{array}$ & $\begin{array}{c}\mathrm{Ca} \\
\text { Trat.Médias }\end{array}$ & $\begin{array}{c}\mathrm{Mg} \\
\text { Trat.Médias }\end{array}$ & $\begin{array}{c}\mathbf{S} \\
\text { Trat.Médias }\end{array}$ & $\begin{array}{l}\text { Produtividade } \\
\text { Trat.Médias }\end{array}$ \\
\hline HC86 30,5a & HC86 1,3a & NV86 13,4a & VV86 42,3a & HC85 4,7a & HC86 3,2a & $\begin{array}{c}\text { tha } \\
\text { HC85 52,22a }\end{array}$ \\
\hline HC85 28,7ab & NV86 1,3a & $\mathrm{NC86} 13,1 \mathrm{a}$ & VC86 40,2a & VC86 3,6a & NC86 2,8ab & VC86 41,44a \\
\hline NC86 26,6 b & PC84 1,3a & HC86 13,1a & NV86 39,9ab & VV86 3,5a & NV86 2,7abc & HC86 37,66ab \\
\hline VC86 26,5 b & NC86 $1,3 a$ & PC84 $12,7 a$ & HC85 39,7abc & NC86 3,5a & VC86 2,7abc & VV86 36,23ab \\
\hline PC84 25,5 bc & VC86 $1,3 a$ & VC86 $12,0 a$ & NC86 39,6abc & HC86 3,4a & PC84 2,7abc & NC86 33,75ab \\
\hline NV86 25,5 bc & VV86 $1,3 a$ & HC85 12,0a & PC86 38,3abc & NV86 3,2a & HC85 2,7abc & PC86 29,73 b \\
\hline VV86 $25,3 \mathrm{bc}$ & PC86 $1,3 a$ & PC86 11,7a & PC84 33,3 bc & PC86 3,0a & PC86 2,6 bc & NV86 29,31 b \\
\hline PC86 $24,4 \mathrm{c}$ & HC85 1,1a & VV86 $11,6 a$ & HC86 30,2 c & PC84 2,9a & VV86 2,4 c & PC84 26,79b \\
\hline
\end{tabular}

Codificação dos tratamentos: HC86 - Hamlin sobre Cravo $(8,0 \times 6,0) m ;$ HC85 - Hamlin sobre Cravo (8,0x5,0)m; NC86 - Natal sobre Cravo $(8,0 \times 6,0) \mathrm{m}$; NV86 - Natal sobre Volkameriana $(8,0 \times 6,0) \mathrm{m}$; PC84 - Pêra sobre Cravo $(8,0 \times 4,0) \mathrm{m}$; PC86 - Pêra sobre Cravo $(8,0 \times 6,0) \mathrm{m}$; VC86 - Valência sobre Cravo $(8,0 \times 6,0) \mathrm{m}$ e VV86 - Valência sobre Volkameriana $(8,0 \times 6,0) \mathrm{m}$;

\subsection{Definição do procedimento padrão de cálculo}

Neste tópico procurou-se estabelecer o procedimento padrão para o cálculo dos indices-DRIS, com o teste das três formas que precederam todas as variações atualmente encontradas na literatura, as proposta por Beaufils (1973) (Beaufils Original - BO), por Elwali \& Gascho (1984) (Beaufils Modificado - BM) e por Jones (1981) (Jones Original - JO).

A equação original de Beaufils (1973) resulta índices DRIS para os nutrientes deficientes maiores, em módulo, realçando assim as deficiências nutricionais. Estas deficiências são importantes do ponto de vista fisiológico, limitantes da produtividade.

A modificação proposta por Elwali \& Gascho (1984), de maneira geral, reduz o valor absoluto do Índice-DRIS, por não incluir pequenos desvios da razão dos teores de nutrientes na amostra em relação a razão média dos teores dos nutrientes na população de referência, nos cálculos. Por este motivo é de se esperar que a diagnose 
por este procedimento de cálculo, tenha uma menor sensibilidade na interpretação do estado nutricional.

Tem-se dado preferência para o equacionamento de Jones (1981) que apresenta uma base na formalidade estatística, tal qual a utilizada normalmente no controle estatístico de qualidade em indústrias, Lourenço Filho (1982).

Para avaliar o melhor procedimento, foram calculados os índices para todos os dados da rede de ensaios de adubação N, P e K. Então, foi calculado o B.N. para cada amostra e este usado no cálculo de correlação com a produtividade, sendo esperada uma correlação negativa, pois quanto maior o B.N., maior é o desequilíbrio entre os nutrientes e menor a produtividade.

Os coeficientes de correlação para cada procedimento de cálculo estão na Tabela 13. Pode-se observar que a tendência da relação esperada entre produtividade e B.N. foi obtida em um número maior de conjunto de dados (locais) analisados, no procedimento proposto por Jones (1981), num total de 4 locais, sendo que a correlação foi significativa em apenas 2. Enquanto que por Beaufils, foi obtida a tendência em apenas 3 , contudo de correlação significativa nos 3 locais.

Tabela 13. Coeficientes de correlação entre produtividade e o B.N. calculado pelos procedimentos de Beaufils e de Jones.

\begin{tabular}{|c|c|c|c|}
\hline LOCAL & $B O^{(l)}$ & $B M^{(2)}$ & $J O^{(3)}$ \\
\hline Araraquara & 0,1081 & 0,1536 & 0,2304 \\
\hline Botucatu & $-0,3365^{* * *}$ & $-0,3322^{* *}$ & $-0,1470$ \\
\hline Matão & $-0,4340^{n k}$ & $-0,4655^{* *}$ & $-0,3602^{k \star}$ \\
\hline Monte Azul & $-0,6432^{n k t}$ & $-0,6920^{\star *}$ & $-0,6003^{* *}$ \\
\hline Olímpia & 0,2008 & 0,2840 & $0,3743^{* * *}$ \\
\hline Pirassununga & 0,0871 & 0,1506 & $-0,1152$ \\
\hline
\end{tabular}

${ }^{71)} \mathrm{BO}$ - Beaufils Original; ${ }^{(2)} \mathrm{BM}$ - Beaufils Modificado $e^{(3)} \mathrm{JO}$ - Jones Original

Com esses resultados é difícil a escolha do melhor procedimento entre os três estudados, sendo por isso também avaliados segundo a interpretação da diagnose dos índices gerados para os dados dos ensaios de adubação N, P e K. 


\subsection{Comportamento dos teores foliares dos macronutrientes}

Com a nova base de dados-referência para citros foi possível correlacionar os teores foliares dos macronutrientes com a produtividade. Na Figura 2, está apresentada a tendência de resposta da produtividade em função do nutriente, e a faixa de teor foliar do nutriente que possibilitou obter as maiores produtividades.

No entanto, é difícil a visualização dessas tendências pela forma com que os dados estão dispersos. Então foram estabelecidas onze faixas de produtividade. Dentro de cada faixa foram calculados os teores foliares médios e a produtividade média (Tabela 14). Com estes dados médios foi elaborada a Figura 3, que mostra com mais nitidez o efeito de cada nutriente sobre a produtividade.

Tabela 14. Teores foliares médios de nutrientes referentes a cada diferente classe de produtividade extraídas da população de citros de Matão/SP.

\begin{tabular}{|c|c|c|c|c|c|c|}
\hline \multirow{2}{*}{$\begin{array}{l}\text { Produtividade } \\
\text { Média }\end{array}$} & \multicolumn{6}{|c|}{ Teores foliares médios de nutrientes } \\
\hline & $\mathrm{N}$ & $\mathbf{P}$ & $\mathrm{K}$ & $\mathrm{Ca}$ & $\mathrm{Mg}$ & $\mathbf{S}$ \\
\hline $\mathrm{tha}^{-1}$ & & & E & & & \\
\hline 16,14 & 25,8 & 1,3 & 11,3 & 37,3 & 3,4 & 1,7 \\
\hline 21,29 & 25,4 & 1,3 & 11,1 & 35,9 & 3,1 & 1,8 \\
\hline 26,45 & 25,5 & 1,3 & 12,4 & 36,6 & 3,2 & 1,9 \\
\hline 31,60 & 25,7 & 1,3 & 12,8 & 38,5 & 3,3 & 2,1 \\
\hline 36,76 & 26,5 & 1,3 & 12,3 & 39,5 & 3,3 & 2,2 \\
\hline 41,91 & 26,3 & 1,2 & 12,8 & 39,7 & 3,4 & 2,4 \\
\hline 47,07 & 25,5 & 1,3 & 11,9 & 41,1 & 3,4 & 1,7 \\
\hline 52,22 & 25,7 & 1,2 & 12,2 & 42,4 & 3,5 & 2,0 \\
\hline 57,38 & 27,2 & 1,1 & 11,8 & 49,4 & 5,9 & 2,7 \\
\hline 62,53 & 26,3 & 1,8 & 12,8 & 45,4 & 4,2 & 0,8 \\
\hline 67,69 & 26,9 & 1,6 & 13,0 & 45,4 & 4,3 & 1,1 \\
\hline
\end{tabular}

As tendências de resposta da produtividade em função dos nutrientes puderam ser quantificadas de forma mais simples através do coeficiente de correlação (Tabela 15). Na Tabela 16 são apresentados os coeficientes de correlação entre os nutrientes e entre nutrientes e produtividade com os dados dos ensaios de adubação $\mathrm{N}, \mathrm{P}$ e $\mathrm{K}$ nas diversas combinações de variedades de copa e porta-enxerto. 
Tabela 15. Matriz de coeficientes de correlação entre produtividade média e teores foliares médios de nutrientes de cada classe de produtividade.

\begin{tabular}{|c|c|c|c|c|c|c|}
\hline & $\boldsymbol{N}$ & $\boldsymbol{P}$ & $\boldsymbol{K}$ & $\mathrm{Ca}$ & $M g$ & $\boldsymbol{S}$ \\
\hline $\bar{P}$ & 0,1053 & & & & & \\
\hline $\boldsymbol{K}$ & 0,3191 & 0,4883 & & & & \\
\hline $\mathrm{Ca}$ & $0,7897 * *$ & 0,2092 & 0,2871 & & & \\
\hline$M g$ & $0,7956 * *$ & $-0,0068$ & 0,0222 & $0,9001^{*}$ & & \\
\hline$S$ & 0,0757 & $-0,9292 * *$ & $-0,2435$ & $-0,0969$ & 0,0933 & \\
\hline Produtividade & $0,6472 *$ & 0,4171 & 0,5488 & $0,8961^{* *}$ & $0,6381^{*}$ & $-0,2973$ \\
\hline
\end{tabular}

*,** coeficientes de correlação significativos aos níveis de 1 e $5 \%$ de probabilidade, respectivamente.

$\mathrm{F}$

f 

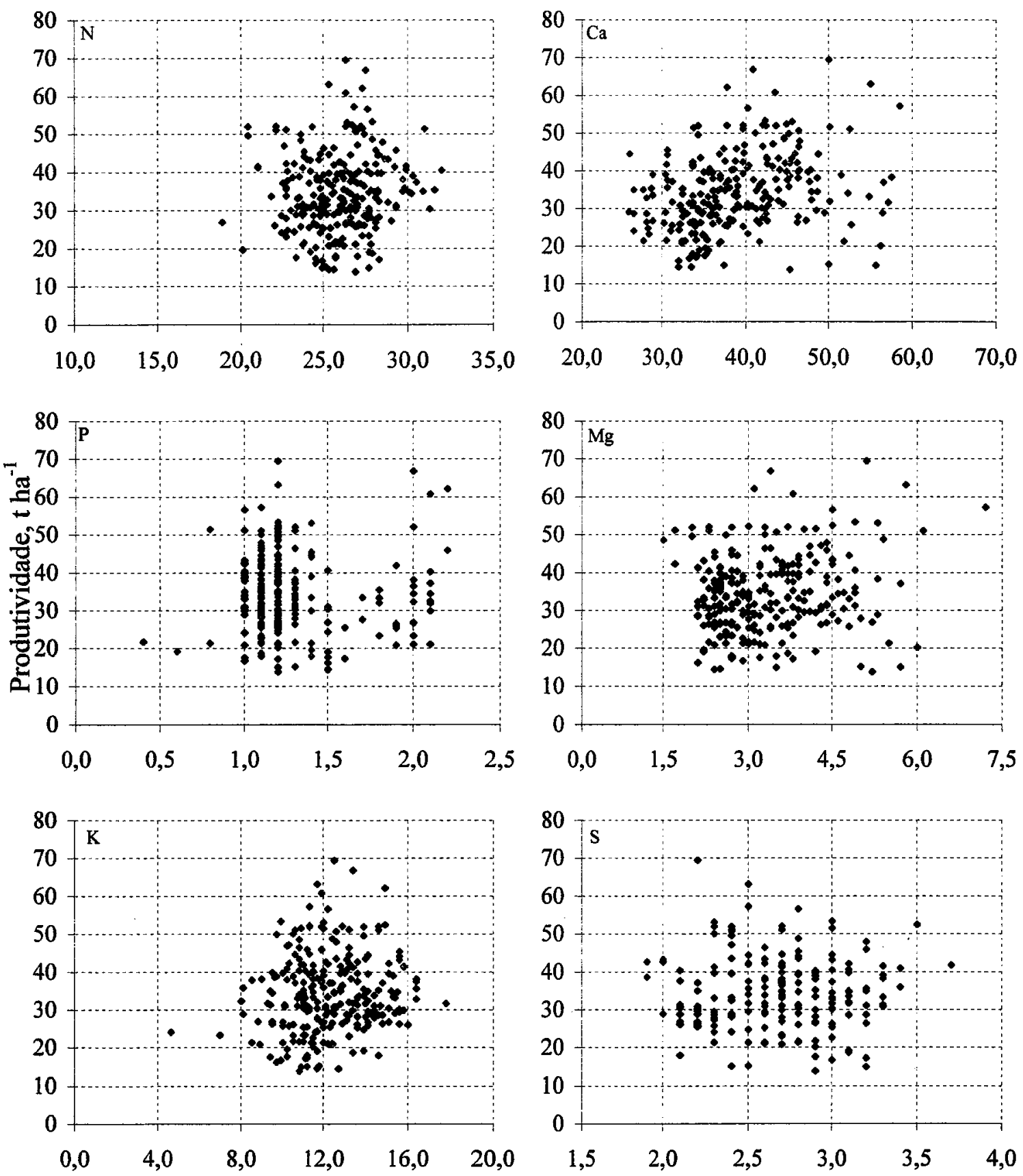

Teor foliar, $\mathrm{g} \mathrm{kg}^{-1}$

Figura 2. Relações entre teores foliares de macronutrientes e produtividade para 0 conjunto de dados da população. 

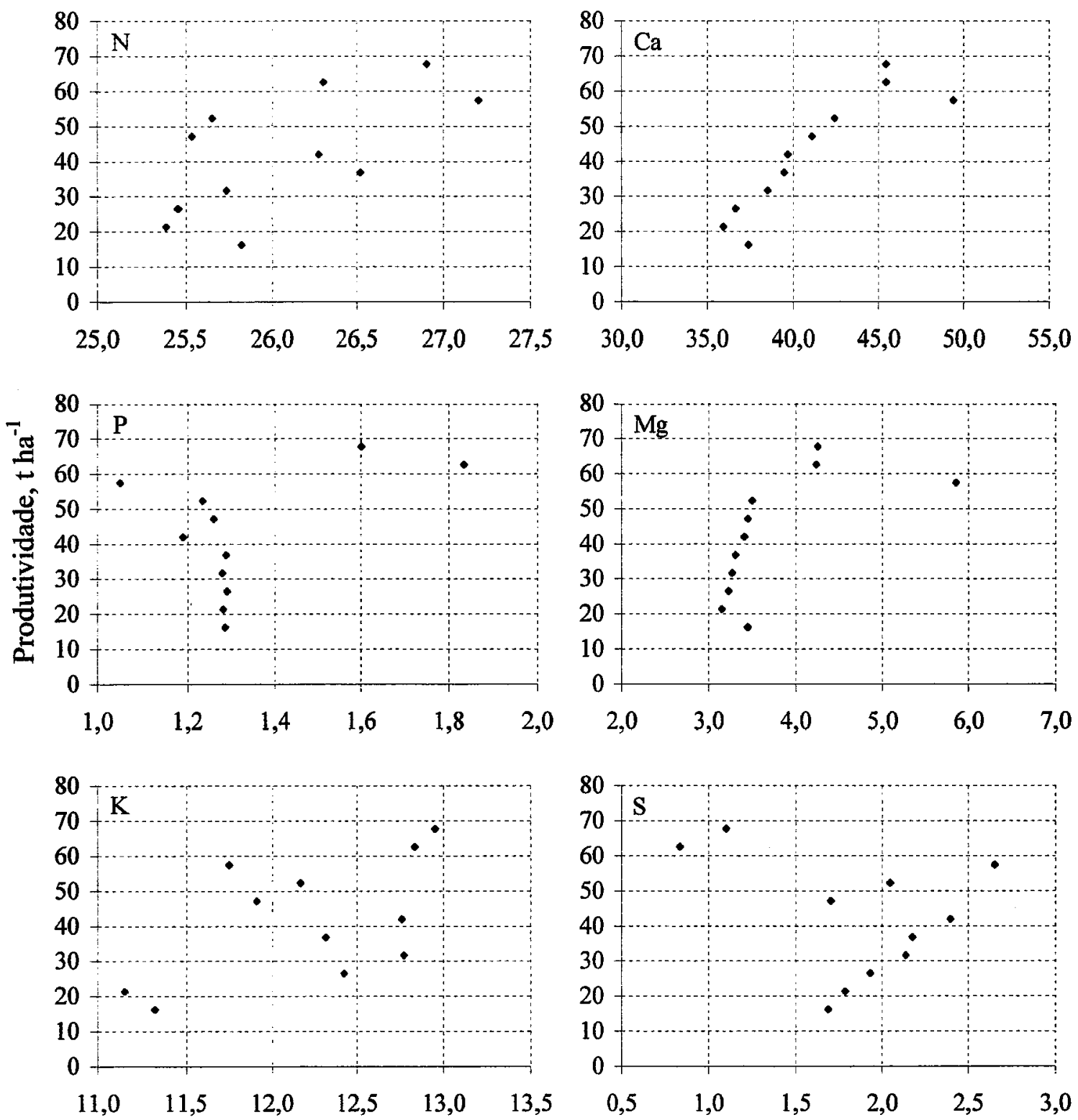

Teor foliar, $\mathrm{g} \mathrm{kg}^{-1}$

Figura 3. Relações entre teores foliares médios dos macronutrientes e a produtividade média das diferentes classes de produtividade. 
a Tabela 15, estão também apresentados os coeficientes de correlação entre os nutrientes, nos quais podem ser observadas várias interações importantes, como:

a) $\mathrm{N} \times \mathrm{Ca}$ : os dois principais nutrientes, em termos de quantidades, nos citros, apresentam correlação positiva, sendo explicada pela forma de absorção do nutriente. $\mathrm{Ou}$ seja, o $\mathrm{N}$ na forma de $\mathrm{NO}_{3}^{-}$, um ânion e o $\mathrm{Ca}$ como $\mathrm{Ca}^{2+}$, um cátion, então, a partir da disponibilidade desses dois nutrientes no solo, um nutriente favorece a entrada (absorção do nutriente pelas raízes) do outro;

b) N x Mg: o mesmo efeito observado com o cálcio foi observado com o magnésio, neste conjunto de dados, pois apesar das quantidades de magnésio absorvido pela planta cítrica ser bem menor, em relação ao cálcio, o mecanismo interativo pode ser 0 mesmo, pois o $\mathrm{Mg}$ é absorvido na forma $\mathrm{Mg}^{2+}$, um cátion;

c) Ca e Mg: o efeito observado desses dois nutrientes pode ter origem pela calagem e os benefícios associados como eliminação de alguma barreira química no subsolo, melhora o desenvolvimento radicular (maior volume de solo a ser explorado pelas raízes) e ação direta sobre a mineralização, principalmente, do nitrogênio orgânico;

d) $\mathrm{N} \mathrm{x} \mathrm{K}$ : as características dos dados considerados mostram que graças a uma condição da adubação mais equilibrada de $\mathrm{N}, \mathrm{K}, \mathrm{Ca}$ e $\mathrm{Mg}$, a interação entre eles é favorável a uma maior absorção, ou seja, o fornecimento de nitrogênio além do papel fisiológico na planta, atua também no mecanismo de absorção dos cátions, $\mathrm{Ca}^{2+}, \mathrm{K}^{+} \mathrm{e} \mathrm{Mg}^{2+}$, estes por sua vez, quando em níveis correspondentes ao do nitrogênio no solo favorecem a entrada do principal ânion. Essas observações sugerem que este mecanismo está baseado no equilíbrio de cargas no interior da planta, como já constatado por Quaggio (1991);

e) Ca x Mg: esta interação positiva de altíssima significância evidencia os cuidados no fornecimento destes dois nutrientes de forma equilibrada e proporcional às necessidades da planta; 
f) P x K: outra interação positiva, contudo ainda sem uma adequada compreensão;

g) P x S: interação negativa, ou seja, de caráter antagônico e de elevada significância nos dados considerados, pode ter origem no fato de que em nenhuma das fontes utilizadas para o fornecimento dos nutrientes via fertilizante, continha o enxôfre na sua constituição;

h) Produtividade $x$ macronutrientes: foi observada dependência direta ou positiva dos cinco principais macronutrientes $(\mathrm{N}, \mathrm{P}, \mathrm{K}, \mathrm{Ca}$ e $\mathrm{Mg}$ ), o que evidencia a necessidade de uma melhor compreensão dos mecanismos interativos entre esse nutrientes e entre esses nutrientes e a produtividade, como já verificada em Quaggio (1991) e Cantarella et al.(1994);

i) $\mathrm{K} \times \mathrm{Ca}, \mathrm{K} \times \mathrm{Mg}, \mathrm{K} \times(\mathrm{Ca}+\mathrm{Mg})$ : estas interações de caráter antagônico, encontradas na literatura e mesmo neste trabalho quando, considera-se os dados do ensaio de adubação N, P e K, quando foram impostas condições de desequilíbrio entre essas bases no solo, o que refletiu também em teores desequilibrados nas folhas. Contudo, com os dados considerados não foi possível obter nem a tendência esperada (correlação negativa, mesmo a nível de significância baixa), o que pode ser uma forma de evidenciar que o fornecimento dos nutrientes está sendo feito de maneira equilibrada, como já realçado em outras interações; e

j) foi possível observar as tendências de interação entre os nutrientes encontradas na literatura, como as de antagonismo entre as bases $(\mathrm{K}, \mathrm{Ca}$ e $\mathrm{Mg})$ e entre $\mathrm{N}$ e $\mathrm{Ca}$ como a verificada por Weir (1969). Esse conjunto de dados representa uma situação que evidencia a necessidade do correto manejo da adubação potássica, bem como dos níveis de cálcio e magnésio. 
Tabela 16. Coeficientes de correlação entre os teores foliares e a produtividade, nas várias situações encontradas na rede de ensaios de adubação $\mathrm{N}, \mathrm{P}$ e K.

\begin{tabular}{|c|c|c|c|c|c|c|c|c|c|}
\hline \multirow[t]{2}{*}{ Interação } & \multicolumn{6}{|c|}{ Locais $^{(1)}$} & \multicolumn{3}{|c|}{ Laranjeira } \\
\hline & I & II & III & IV & $\mathrm{V}$ & $\overline{V I}$ & Geral & Pêra $\mathrm{F}$ & Pêra/Cravo \\
\hline$\overline{\mathrm{NxP}}$ & $-0,2750^{*}$ & $0,5562^{* *}$ & $-0,2717^{*}$ & $-0,5432^{3 *}$ & 0,0091 & 0,1627 & 0,0656 & 0,0605 & $-0,3495^{* *}$ \\
\hline NxK & $-0,4603^{* *}$ & $-0,3351^{* *}$ & $-0,0415$ & $-0,1325$ & 0,0314 & $-0,3934^{* *}$ & 0,2014 & $0,4056^{* *}$ & * $0,3895^{* *}$ \\
\hline $\mathrm{NxCa}$ & $0,7463^{* *}$ & 0,1285 & $-0,3025^{* *}$ & $-0,1057$ & 0,1607 & $-0,3270^{* *}$ & $-0,7003^{* *}$ & $-0,7406^{* *}$ & $-0,7307^{* *}$ \\
\hline $\mathrm{NxMg}$ & $0,3475^{* *}$ & $0,3575^{* *}$ & $-0,2380$ & $-0,1925$ & $-0,0230$ & $0,4873^{* *}$ & $0,3701^{* *}$ & $-0,4787^{* *}$ & $-0,4920^{* *}$ \\
\hline $\mathrm{NxS}$ & $-0,4883^{* *}$ & $-0,7063^{* *}$ & 0,1090 & $-0,6690^{* *}$ & $-0,1372$ & $-0,3332^{* *}$ & $0,6591^{* *}$ & $0,2833^{*}$ & 0,0100 \\
\hline NxProd & $0,5141^{* *}$ & $0,4516^{* *}$ & $0,3478^{* *}$ & $0,6484^{* *}$ & 0,0893 & $-0,0086$ & $0,3793^{* *}$ & $-0,1253$ & $-0,2511^{*}$ \\
\hline PxK & 0,1676 & $-0,3172^{*}$ & 0,1245 & $0,6051^{* *}$ & $0,7638^{* *}$ & $0,3588^{* *}$ & $0,3438^{* *}$ & $0,5818^{* *}$ & * $0,3011^{*}$ \\
\hline $\mathrm{PxCa}$ & $-0,4742^{* *}$ & $0,4155^{* *}$ & $-0,1387$ & $-0,5285^{* *}$ & $0,4020^{* *}$ & $-0,4034^{* *}$ & $-0,3380^{* *}$ & $-0,3804^{* *}$ & 0,0048 \\
\hline PxMg & 0,1398 & $0,3878^{* *}$ & $-0,0002$ & $-0,1198$ & $0,3573^{* *}$ & $-0,3444^{* *}$ & $-0,3062^{*}$ & $-0,5513^{* *}$ & 0,0478 \\
\hline PxS & $0,4695^{* *}$ & $-0,4159^{* *}$ & $-0,2410$ & $0,6436^{* *}$ & $0,6075^{* *}$ & $-0,2249$ & $0,4140^{* *}$ & $0,6423^{* *}$ & * $0,4878^{* *}$ \\
\hline PxProd & $-0,2079$ & $0,5654^{* *}$ & $-0,1569$ & $-0,8023^{* *}$ & $-0,4818^{* *}$ & $-0,6591^{* *}$ & $-0,1758$ & $-0,3192^{*}$ & $-0,4885^{* *}$ \\
\hline $\mathrm{KxCa}$ & $-0,6015^{* *}$ & $-0,9217^{* *}$ & $-0,6249^{* *}$ & $-0,5917^{* *}$ & 0,0257 & $-0,6624^{* *}$ & $-0,3754^{* *}$ & $-0,8007^{* *}$ & ${ }^{*}-0,7253^{* *}$ \\
\hline $\mathrm{KxMg}$ & $-0,7094^{* *}$ & $-0,9566^{* *}$ & $-0,7994^{* *}$ & 0,1104 & 0,0482 & $-0,8360^{* *}$ & $-0,3190^{*}$ & $-0,8178^{* *}$ & ${ }^{*}-0,5669^{* *}$ \\
\hline $\mathrm{KxS}$ & $-0,0320$ & $0,5198^{* *}$ & $-0,0238$ & $0,4133^{* *}$ & $0,3756^{* *}$ & 0,1486 & 0,1677 & $0,6714^{* *}$ & $0,4471^{* *}$ \\
\hline KxProd & $0,2635^{*}$ & $0,2563^{*}$ & $-0,1619$ & $-0,2435$ & $-0,1965$ & $-0,1762$ & $0,4755^{* *}$ & $-0,1757$ & $-0,4851^{* *}$ \\
\hline $\mathrm{CaxMg}$ & $0,3019^{*}$ & $0,9334^{* *}$ & $0,8197^{* *}$ & 0,1710 & $0,8686^{* *}$ & $0,3961^{* *}$ & 0,1063 & $0,7438^{* *}$ & $0,6847^{* *}$ \\
\hline CaxS & $-0,1877$ & $-0,2985^{*}$ & $0,5255^{* *}$ & $-0,0971$ & $0,7125^{* *}$ & $-0,0258$ & $-0,6604^{* *}$ & $-0,6552^{* *}$ & $-0,4295^{* *}$ \\
\hline CaxProd & $0,2523^{*}$ & $-0,2246$ & $0,2763^{*}$ & 0,2370 & $-0,6468^{* *}$ & 0,0769 & 0,0325 & $0,3768^{* *}$ & $0,6539^{* *}$ \\
\hline MgxS & 0,0297 & $-0,4175^{* *}$ & 0,1510 & $0,3318^{* *}$ & $0,7986^{* *}$ & 0,0234 & $0,2897^{*}$ & $-0,6785^{* *}$ & * $-0,1378$ \\
\hline MgxProd & $-0,1130$ & $-0,2987^{*}$ & $-0,0285$ & $0,3013^{*}$ & $-0,6248^{* *}$ & $0,4347^{* *}$ & $0,3765^{* *}$ & 0,0304 & $0,5148^{* *}$ \\
\hline SxProd & $-0,7687^{* *}$ & $-0,2984^{*}$ & 0,0986 & $-0,7055^{* *}$ & $-0,4700^{* *}$ & 0,1037 & 0,0981 & $-0,3738^{* *}$ & ${ }^{*}-0,7473^{* *}$ \\
\hline
\end{tabular}




\subsection{Comportamento nutricional do limoeiro Siciliano (Citrus limon (L.) Burmann)}

A adubação do limoeiro Siciliano (Citrus limon (L.) Burmann) com diferentes doses de nitrogênio, fỏsforo e potássio, possibilitou obter teores foliares típicos dos macronutrientes, os quais podem ser vistos na Tabela 17. Nessa mesma Tabela observa-se o comportamento diferencial do limoeiro Siciliano em relação aos demais citros estudados, que são:

. produtividade do limoeiro é altíssima, em termos de caixas/planta ou em toneladas/hectare, e ainda assim, responde positivamente a adubação N, $\mathrm{P}$ e K;

. teor médio de potássio encontrado nas folhas é, em geral, a metade do teor médio nas demais variedades estudadas;

. a variação dos teores foliares, quantificada através do coeficiente de variação, foi muito maior que a normal para os nutrientes potássio, cálcio e magnésio, talvez devido a alta resposta do limoeiro em elevar os teores de potássio em função da adubação potássica, logo pelo antagonismo existente, principalmente entre o potássio, cálcio e magnésio, os teores foliares do cálcio e do magnésio também sofrem variação.

Estas observações sugerem que o adequado fornecimento de adubos potássicos segue um padrão diferente de outras variedades cítricas, graças a sua demanda metabólica diferenciada.

Tabela 17. Parâmetros estatísticos dos teores foliares e produção do limoeiro siciliano.

\begin{tabular}{lrrrrrrc}
\hline & $\mathbf{N}$ & $\mathbf{P}$ & $\mathbf{K}$ & $\mathbf{C a}$ & $\mathbf{M g}$ & $\mathbf{S}$ & PROD \\
\cline { 2 - 6 } médio & 20,5 & 1,4 & 7,6 & 8,8 & 5,3 & 2,2 & 47,96 \\
mínimo & 17,2 & 1,2 & 3,2 & 6,7 & 3,6 & 1,6 & 33,80 \\
máximo & 23,6 & 1,6 & 5,1 & 4,5 & 7,4 & 2,8 & 58,10 \\
desvio padrão & 1,8 & 0,1 & 3,7 & 6,8 & 1,0 & 0,4 & 6,74 \\
coef.variação, \% & 8,8 & 9,2 & 48,9 & 17,5 & 19,0 & 16,6 & 14,1 \\
\hline
\end{tabular}




\subsection{Interpretação do estado nutricional nos ensaios de adubação com base na população de referência para citros.}

A avaliação da eficiência tanto da diagnose da nova população como dos procedimentos de cálculo podem ser feitas nas sequiências de correção pela adubação nos casos em que desequilíbrios nutricionais foram evidenciados pelos índices-DRIS. Seguindo-se os procedimentos propostos por Beverly \& Hallmark (1992), como exemplo, foram classificadas as diagnoses pelo procedimento proposto por Jones (1981) para N, P e K, e determinadas as respostas da produtividade à correção para cada um dos três nutrientes, no ensaio de adubação $\mathrm{N}, \mathrm{P}$ e K conduzido em Matão/SP (Tabelas 18 e 19). $\mathrm{O}$ mesmo procedimento foi adotado para os outros cinco locais experimentais e para os outros três métodos em avaliação.

Os valores estatísticos de referência para cada local da rede de ensaios de adubação N, P e K e para duas populações extraídas de pomares comerciais da região de Matão/SP estão apresentados nos apêndices de 1 a 8 . Enquanto que os índices DRIS gerados pelos métodos em avaliação estão nos apêndices 9 a 44 , juntamente com a diagnose do estado nutricional e o desempenho da diagnose. Nos apêndices 45 a 56 estão apresentadas a classificação dos teores foliares dos macronutrientes pelo Critério de Faixas de Suficiência acompanhadas do desempenho da diagnose.

Após a totalização do número de casos em que cada método foi bem e mal sucedido na diagnose e recomendação da adubação para os nutrientes $\mathrm{N}, \mathrm{P}$ e K, pode-se calcular o percentual de casos classificados em $\mathrm{V}^{+}, \mathrm{V}^{-}, \mathrm{F}^{+}$e $\mathrm{F}^{-}$, bem como totalizar as respostas de produtividade à correção (Tabela 20).

$\mathrm{Na}$ Tabela 21, são apresentados os índices quantitativos da eficiência dos métodos diagnósticos para os citros em geral, nos seis locais da rede de ensaios fatoriais de adubação. 
Tabela 18. Índices DRIS, calculados pelo procedimento proposto por Jones (1981), diagnose para a correção de $\mathrm{N}, \mathrm{P}$ e $\mathrm{K}$, resposta líquida da produtividade, diagnose para o nutriente deficiente e a resposta da produtividade, para os dados, dos tratamentos com as doses 0 e 1 de $\mathrm{N}$ em todas as combinações de doses de P e K, do ensaio de adubação N, P e K conduzido em Matão/SP.

\begin{tabular}{|c|c|c|c|c|c|c|c|c|c|c|c|c|c|c|}
\hline \multirow[b]{2}{*}{ Trat. } & \multicolumn{6}{|c|}{ Índices DRIS } & \multirow[b]{2}{*}{$\mathrm{BN}$} & \multirow[b]{2}{*}{ Prod. } & \multicolumn{3}{|c|}{ Diagnose } & \multicolumn{3}{|c|}{ Resposta } \\
\hline & $\overline{\mathrm{N}}$ & $\bar{P}$ & $\bar{K}$ & $\mathrm{Ca}$ & $\overline{\mathrm{Mg}}$ & $\bar{S}$ & & & $\bar{N}$ & $\mathrm{P}$ & $\bar{K}$ & $d\left(Y_{N}\right)$ & $d\left(Y_{P}\right)$ & $\bar{d}\left(Y_{K}\right)$ \\
\hline & & & & & & & & tha $\mathrm{A}^{-1}$ & & & & & W & \\
\hline 000 & -83 & -21 & 45 & 7 & 98 & -46 & 50 & 34,77 & $\mathbf{V}^{+}$ & $\mathrm{V}^{+}$ & $\mathrm{V}^{-}$ & 2,21 & 4,75 & 1,54 \\
\hline 001 & -91 & 0 & 99 & -28 & 72 & -52 & 57 & 33,23 & $\mathrm{~V}^{+}$ & $F^{*}$ & $F^{-}$ & 2,04 & $-4,34$ & $-0,35$ \\
\hline 002 & -76 & 1 & 154 & -52 & 45 & -72 & 67 & 33,58 & $\mathrm{~V}^{+}$ & $\mathrm{F}^{-}$ & $\mathrm{F}^{-}$ & 1,95 & $-3,90$ & $-2,21$ \\
\hline 003 & -55 & 15 & 201 & -77 & $17-$ & -101 & 78 & 35,79 & $\mathrm{~V}^{+}$ & $\mathrm{F}^{-}$ & - & 1,86 & $-3,48$ & - \\
\hline 010 & -98 & 9 & 59 & -1 & 95 & -64 & 54 & 39,52 & $\mathrm{~V}^{+}$ & $\mathrm{F}^{*}$ & $\mathrm{~V}^{-}$ & 2,30 & $-2,47$ & 1,95 \\
\hline 011 & -83 & 0 & 109 & -21 & 69 & -75 & 60 & 37,57 & $\mathrm{~V}^{+}$ & $\mathrm{F}^{-}$ & $\mathrm{V}^{-}$ & 2,13 & $-2,04$ & 0,09 \\
\hline 012 & -80 & 31 & 141 & -52 & 45 & -86 & 73 & 37,48 & $\mathrm{~V}^{+}$ & $\mathrm{F}^{*}$ & $\mathrm{~F}^{-}$ & 2,04 & $-1,54$ & $-1,79$ \\
\hline 013 & -56 & 46 & 179 & -74 & $19-$ & -114 & 81 & 39 , & $\mathrm{V}^{+}$ & $\mathrm{F}^{-}$ & - & 96 & $-1,11$ & - \\
\hline 020 & -99 & 6 & 60 & 8 & 93 & -67 & 56 & 41,99 & $\mathrm{~V}^{+}$ & $\mathrm{F}^{-}$ & $\mathrm{V}^{-}$ & 2,38 & $-0,09$ & 2,38 \\
\hline 021 & -88 & -8 & 95 & -13 & 74 & -60 & 56 & 39,61 & $\mathrm{~V}^{+}$ & $\mathrm{F}^{+}$ & $\mathrm{V}^{*}$ & 2,21 & $-0,34$ & 0,59 \\
\hline 022 & -80 & 26 & 115 & -41 & 51 & -71 & 64 & 39,02 & $\mathrm{~V}^{+}$ & $\mathrm{V}^{-}$ & $\mathrm{F}^{-}$ & 2,12 & 0,77 & $-1,36$ \\
\hline 023 & -54 & 43 & 145 & -59 & 24 & -99 & 71 & 40,38 & $\mathrm{~V}^{+}$ & $\mathrm{V}^{-}$ & - & 2,03 & 1,19 & - \\
\hline 030 & -99 & -41 & 44 & 29 & 106 & -39 & 60 & 42,08 & $\mathrm{~V}^{+}$ & - & $\mathrm{V}^{-}$ & 2,54 & - & 2,81 \\
\hline 031 & -89 & -12 & 59 & 2 & 82 & -42 & 48 & 39,27 & $\mathrm{~V}^{+}$ & - & $\mathrm{V}^{-}$ & 2,29 & - & 1,02 \\
\hline 032 & -71 & -11 & 78 & -16 & 63 & -43 & 47 & 38,25 & $\mathrm{~V}^{+}$ & - & $F^{-}$ & 2,29 & - & $-0,94$ \\
\hline 033 & -48 & 2 & 93 & -36 & 43 & -54 & 46 & 39,19 & $\mathrm{~V}^{+}$ & - & - & 2,12 & - & - \\
\hline 100 & -28 & 26 & -27 & -3 & 104 & -73 & 44 & 36,98 & $\mathrm{~V}^{+}$ & $\mathrm{F}^{*}$ & $\mathrm{~F}^{+}$ & 1,01 & $-4,84$ & $-1,71$ \\
\hline 101 & -37 & 7 & 48 & -27 & 81 & -72 & 45 & 35,27 & $\mathrm{~V}^{+}$ & $\mathrm{F}^{-}$ & $\mathrm{F}^{-}$ & 0,94 & $-4,43$ & $-0,26$ \\
\hline 102 & -35 & 2 & 110 & -50 & 50 & -77 & 54 & 35,53 & $\mathrm{~V}^{+}$ & $\mathrm{F}^{-}$ & $\mathrm{F}^{-}$ & 0,85 & $-3,99$ & $-2,12$ \\
\hline 103 & -23 & 13 & 163 & -73 & & -110 & 69 & 37,65 & $\mathrm{~V}^{+}$ & $\mathrm{F}^{-}$ & - & 0,69 & $-3,58$ & - \\
\hline 110 & -33 & 22 & 15 & -1 & 98- & -102 & 45 & 41,82 & $\mathrm{~V}^{+}$ & $\mathrm{F}^{-}$ & $\mathrm{V}^{-}$ & 1,19 & $-2,55$ & 2,12 \\
\hline 111 & -41 & 1 & 68 & -26 & 76 & -78 & 48 & 39,70 & $\mathrm{~V}^{+}$ & $\mathrm{F}^{-}$ & $\mathrm{V}^{-}$ & 1,10 & $-2,12$ & 0,18 \\
\hline 112 & -36 & 35 & 109 & -51 & $52-$ & -109 & 65 & 39,52 & $\mathrm{~V}^{+}$ & $F^{-}$ & $\mathrm{F}^{-}$ & 0,94 & $-1,62$ & $-1,71$ \\
\hline 113 & -17 & 11 & 158 & -67 & $28-$ & -113 & 66 & 41,23 & $\mathrm{~V}^{+}$ & $\mathrm{F}^{-}$ & - & 0,76 & $-1,18$ & - \\
\hline 120 & -47 & 13 & 25 & 2 & 93 & -87 & 45 & 44,37 & $\mathrm{~V}^{+}$ & $\mathrm{F}^{-}$ & $\mathrm{V}^{-}$ & 1,28 & $-0,25$ & 2,55 \\
\hline 121 & -42 & -2 & 66 & -17 & 74 & -80 & 47 & 41,82 & $\mathrm{~V}^{+}$ & $\mathrm{F}^{+}$ & $\mathrm{V}^{-}$ & 1,19 & $-0,26$ & 0,68 \\
\hline 122 & -39 & 30 & 93 & -42 & 50 & -92 & 58 & 41,14 & $\mathrm{~V}^{+}$ & $\mathrm{V}^{-}$ & $\mathrm{F}^{-}$ & 1,10 & 0,60 & $-1,27$ \\
\hline 123 & -25 & 40 & 124 & -62 & 29 - & -106 & 64 & 42,41 & $\mathrm{~V}^{+}$ & $\mathrm{V}^{-}$ & - & 0,94 & 1,10 & - \\
\hline 130 & -47 & -32 & 18 & 21 & 98 & -57 & 46 & 44,62 & $\mathrm{~V}^{+}$ & - & $\mathrm{V}^{-}$ & 1,37 & - & 3,06 \\
\hline 131 & -52 & -12 & 36 & -7 & 80 & -45 & 39 & 41,56 & $\mathrm{~V}^{+}$ & - & $\mathrm{V}^{-}$ & 1,28 & - & 1,02 \\
\hline 132 & -39 & -13 & 62 & -23 & 60 & -47 & 41 & 40,54 & $\mathrm{~V}^{+}$ & - & $\mathrm{F}^{-}$ & 1,11 & - & $-0,77$ \\
\hline 133 & -22 & 0 & 82 & -40 & 40 & -59 & 41 & 41,31 & $\mathrm{~V}^{+}$ & - & - & 1,02 & - & - \\
\hline
\end{tabular}


Tabela 19. Índices DRIS, calculados pelo procedimento proposto por Jones (1981), diagnose para a correção de $\mathrm{N}, \mathrm{P}$ e $\mathrm{K}$, resposta líquida da produtividade, diagnose para o nutriente deficiente e a resposta da produtividade, para os dados, dos tratamentos com as doses 2 e 3 de $\mathrm{N}$ em todas as combinações de doses de $\mathrm{P}$ e K, do ensaio de adubação N, P e K conduzido em Matão/SP.

Índices DRIS

Trat. $\mathrm{N} \quad \mathrm{P} \quad \mathrm{K} \mathrm{Ca} \mathrm{Mg} \mathrm{S} \mathrm{BN} \mathrm{Prod.}$

\begin{tabular}{|c|c|c|c|c|c|c|c|c|c|c|c|c|c|}
\hline \multirow[b]{2}{*}{311} & \multirow[b]{2}{*}{22} & \multirow[b]{2}{*}{27} & \multirow[b]{2}{*}{-71} & \multirow[b]{2}{*}{-10} & \multicolumn{6}{|c|}{ that } & \multicolumn{3}{|c|}{$\mathrm{tha}^{-1}$} \\
\hline & & & & & $106-73$ & 52 & 37,99 & $\mathrm{~V}^{-}$ & $\mathrm{F}^{-}$ & $\mathrm{F}^{+}$ & - & $-5,02$ & $-1,7$ \\
\hline 312 & 3 & 6 & 20 & -32 & $79 \quad-75$ & 36 & 36,21 & $\mathrm{~V}^{-}$ & $\mathrm{F}^{-}$ & $\mathrm{F}^{-}$ & 0,09 & $-4,59$ & $-0,1$ \\
\hline 313 & 3 & 3 & 92 & -52 & $57-103$ & 52 & 36,38 & $\mathrm{~V}^{-}$ & $F^{-}$ & $\mathrm{F}^{-}$ & 0,26 & $-4,08$ & $-1,9$ \\
\hline 314 & 14 & -32 & 155 & -68 & $40-109$ & 70 & 38,34 & $\mathrm{~V}^{-}$ & $\mathrm{V}+$ & - & 0,35 & 3,65 & \\
\hline 321 & 12 & 23 & -7 & -12 & $89-105$ & 41 & 43,01 & $\mathrm{~F}^{*}$ & $\mathrm{~F}^{-}$ & $\mathrm{F}^{+}$ & $-0,08$ & $-2,64$ & $-2,21$ \\
\hline 322 & 3 & 3 & 54 & -32 & $74-102$ & 45 & 40,80 & $\mathrm{~V}^{-}$ & $\mathrm{F}^{-}$ & V & - & $-2,21$ & 0 , \\
\hline 323 & 3 & -3 & 105 & -51 & $54-107$ & 54 & 40,46 & $\mathrm{~V}^{-}$ & $\mathrm{V}+$ & $\mathrm{F}$ & 0,17 & 1,78 & $-1,5$ \\
\hline 324 & 8 & 4 & 151 & -73 & $32-122$ & 65 & 41,99 & $v$ & $\mathrm{~F}^{-}$ & - &, 26 & $-1,36$ & \\
\hline 331 & -5 & 14 & 16 & -10 & $\begin{array}{ll}74 & -90\end{array}$ & 35 & 45,65 & $\mathrm{~V}^{+}$ & $\mathrm{F}^{-}$ & $\mathrm{V}^{-}$ & 0,16 & $-0,34$ & 2 , \\
\hline 332 & -8 & -4 & 62 & -27 & $\begin{array}{ll}63 & -85\end{array}$ & 42 & 43,01 & $\mathrm{~V}^{+}$ & $\mathrm{F}^{+}$ & $\mathrm{V}^{-}$ & 0,08 & $-0,17$ & 0,7 \\
\hline 333 & -4 & -10 & 100 & -45 & $\begin{array}{lll}50 & -91\end{array}$ & 50 & 42,24 & $\mathrm{~F}^{+}$ & $\mathrm{F}^{+}$ & $\mathrm{F}^{-}$ & $-0,08$ & $-0,59$ & $-1,11$ \\
\hline 334 & 6 & -1 & 133 & -63 & $29-105$ & 56 & 43, & $\mathrm{~V}^{-}$ & $\mathrm{F}^{+}$ & - & 0,17 & $-1,02$ & \\
\hline 341 & -9 & -32 & 18 & 6 & $78-61$ & 34 & 45,99 & $\mathrm{~V}^{+}$ & - & $\mathrm{V}^{-}$ & 0,25 & - & 3 , \\
\hline 342 & -22 & -13 & 42 & -19 & $\begin{array}{ll}59 & -48\end{array}$ & 34 & 42,84 & $\mathrm{~V}^{+}$ & - & $\mathrm{V}^{-}$ & 0,17 & 一 & 1, \\
\hline 343 & -14 & -16 & 69 & -34 & $\begin{array}{ll}48 & -52\end{array}$ & 39 & 41,65 & $\mathrm{~F}^{+}$ & - & $\mathrm{F}^{*}$ & - & - & $-0,6$ \\
\hline 344 & 1 & -6 & 93 & -48 & $26-66$ & 40 & 42,33 & $\mathrm{~V}^{-}$ & - & - & 0,09 & 一 & \\
\hline 411 & 62 & 24 & -75 & -24 & $\begin{array}{lll}93 & -80\end{array}$ & 60 & 37,99 & - & $\mathrm{F}^{-}$ & $\mathrm{F}^{+}$ & - & $-5,10$ & -1, \\
\hline 412 & 33 & 1 & 18 & -43 & $\begin{array}{lll}74 & -83\end{array}$ & 42 & 36,12 & - & $\mathrm{F}^{-}$ & $\mathrm{V}^{-}$ & - & $-4,68$ & \\
\hline 413 & 32 & -50 & 98 & -55 & $\begin{array}{lll}56 & -81\end{array}$ & 62 & 36,12 & - & $\mathrm{V}^{+}$ & $\mathrm{F}^{-}$ & - & 4,17 & $-1,8$ \\
\hline 414 & 29 & -46 & 160 & -76 & $\begin{array}{ll}33 & -99\end{array}$ & 74 & 37,99 & - & $\mathrm{V}^{+}$ & - & - & 3,74 & \\
\hline 421 & 38 & 15 & -3 & -31 & $\begin{array}{lll}74 & -93\end{array}$ & 42 & 43,09 & - & $\mathrm{F}^{-}$ & $\mathrm{F}^{+}$ & - & $-2,72$ & $-2,2$ \\
\hline 422 & 22 & -7 & 63 & -48 & $\begin{array}{ll}60 & -90\end{array}$ & 48 & 40,80 & - & $\mathrm{V}^{+}$ & $\mathrm{V}^{-}$ & - & 2,29 & 0 \\
\hline 423 & 18 & -14 & 118 & -65 & $\begin{array}{ll}39 & -96\end{array}$ & 58 & 40,29 & - & $\mathrm{V}^{+}$ & $\mathrm{F}^{-}$ & - & 1,87 & $-1,4$ \\
\hline 424 & 32 & -50 & 173 & -76 & $22-101$ & 76 & 41,73 & - & $\mathrm{V}^{+}$ & 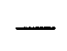 & - & 1,45 & \\
\hline 43 & 26 & 11 & 34 & -27 & $\begin{array}{ll}52 & -97\end{array}$ & 41 & 45,81 & - & $\mathrm{F}^{-}$ & $\mathrm{V}^{-}$ & - & $-0,43$ & 2, \\
\hline 432 & 12 & -13 & 80 & -45 & $\begin{array}{lll}39 & -74\end{array}$ & 44 & 43,09 & - & $\mathrm{F}^{+}$ & $\mathrm{V}^{-}$ & - & $-0,08$ & 0 \\
\hline 433 & 11 & -20 & 119 & -60 & $\begin{array}{ll}28 & -78\end{array}$ & 53 & 42,16 & - & $\mathrm{F}^{+}$ & $\mathrm{F}^{-}$ & - & $-0,51$ & $-1,02$ \\
\hline 43 & 25 & -56 & 164 & -69 & $\begin{array}{lll}19 & -83\end{array}$ & 69 & 43 & - & $\mathrm{F}^{+}$ & - & - & $-0,94$ & \\
\hline 44 & 14 & -40 & 47 & -15 & $44-50$ & 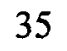 & 46,24 & - & - & $\mathrm{V}^{-}$ & - & - & 3,2 \\
\hline 442 & 5 & -67 & 81 & -33 & $\begin{array}{ll}41 & -27\end{array}$ & 42 & 43,01 & - & - & $\mathrm{V}^{-}$ & - & - & 1,2 \\
\hline 443 & 7 & -71 & 109 & -44 & $\begin{array}{ll}30 & -30\end{array}$ & 49 & 41,65 & - & - & $F^{-}$ & - & - & $-0,59$ \\
\hline 444 & 13 & -66 & 133 & -59 & $\begin{array}{ll}5 & -26\end{array}$ & 50 & 42,24 & - & - & - & - & - & -7 \\
\hline
\end{tabular}


Tabela 20. Valores percentuais do total de casos em que a diagnose da necessidade de cada um dos três nutrientes $(\mathrm{N}, \mathrm{P}$ ou $\mathrm{K})$ em cada um dos seis locais da rede de ensaio de adubação $\mathrm{N}, \mathrm{P}$ e K pelos quatro métodos em avaliação resultou em um incremento da produtividade ou que a produtividade permaneceu inalterada ou foi reduzida.

\begin{tabular}{|c|c|c|c|c|c|c|c|c|}
\hline $\mathrm{R}$ & comendação & & & & & & & \\
\hline Local & pela & Método & Ina & da ou & uzida & & eme & \\
\hline & diagnose & & $\mathrm{N}$ & $P$ & $\mathrm{~K}$ & $\overline{\mathrm{N}}$ & $\mathbf{P}$ & $\bar{K}$ \\
\hline 1 & Fertilizante & CFS & 21 & 50 & 23 & 0 & 0 & 0 \\
\hline 1 & Aplicado & BM & 21 & 50 & 23 & 10 & 0 & 0 \\
\hline - & & BO & 21 & 50 & 23 & 25 & 0 & 0 \\
\hline - & & JO & 21 & 50 & 23 & 29 & 0 & 0 \\
\hline - & Fertilizante & CFS & 79 & 50 & 77 & 0 & 0 & 0 \\
\hline - & não & $\mathrm{BM}$ & 69 & 50 & 77 & 0 & 0 & 0 \\
\hline - & Aplicado & BO & 54 & 50 & 77 & 0 & 0 & 0 \\
\hline - & & JO & 50 & 50 & 77 & 0 & 0 & 0 \\
\hline 2 & Fertilizante & CFS & 4 & 0 & 8 & 75 & 0 & 83 \\
\hline- & Aplicado & $\mathrm{BM}$ & 8 & 0 & 6 & 75 & 0 & 83 \\
\hline - & & $\mathrm{BO}$ & 8 & 0 & 4 & 75 & 0 & 83 \\
\hline - & & $\mathrm{JO}$ & 8 & 0 & 2 & 73 & 0 & 83 \\
\hline - & Fertilizante & $\overline{\mathrm{CFS}}$ & $\frac{\pi}{2}$ & 100 & 0 & 19 & 0 & 8 \\
\hline - & กฮ̃o & BM & 2 & 100 & 0 & 15 & 0 & 10 \\
\hline - & Aplicado & $\mathrm{BO}$ & 2 & 100 & 0 & 15 & 0 & 13 \\
\hline - & & JO & 4 & 100 & 0 & 15 & 0 & 15 \\
\hline 3 & Fertilizante & CFS & 23 & 25 & 60 & 0 & 0 & 0 \\
\hline - & Aplicado & $\mathrm{BM}$ & 6 & 19 & 56 & 65 & 15 & 0 \\
\hline - & & $\mathrm{BO}$ & 8 & 15 & 50 & 75 & 15 & 0 \\
\hline - & & $\mathrm{JO}$ & 19 & 8 & 50 & 75 & 17 & 0 \\
\hline - & Fertilizante & CFS & 77 & 75 & 40 & 0 & 0 & 0 \\
\hline - & não & $\mathrm{BM}$ & 13 & 60 & 40 & 17 & 6 & 4 \\
\hline - & Aplicado & BO & 2 & 60 & 40 & 15 & 10 & 10 \\
\hline - & & JO & 2 & 58 & 40 & 4 & 17 & 10 \\
\hline 4 & Fertilizante & CFS & 10 & $\overline{44}$ & 52 & 0 & $\overline{0}$ & $\overline{0}$ \\
\hline- & Aplicado & $\mathrm{BM}$ & 2 & 38 & 46 & 56 & 0 & 2 \\
\hline$\overline{-}$ & & BO & 0 & 38 & 31 & 83 & 0 & 29 \\
\hline - & & $\mathrm{JO}$ & 0 & 38 & 31 & 81 & 0 & 27 \\
\hline - & Fertilizante & CFS & 90 & $\frac{15}{56}$ & 46 & 0 & 0 & $\frac{1}{2}$ \\
\hline - & não & $\mathrm{BM}$ & 33 & 56 & 44 & 8 & 6 & 8 \\
\hline 一 & Aplicado & $\mathrm{BO}$ & 6 & 56 & 17 & 10 & 6 & 23 \\
\hline - & & JO & 8 & 56 & 19 & 10 & 6 & 23 \\
\hline 5 & Fertilizante & CFS & 44 & 50 & 46 & 0 & 0 & 6 \\
\hline - & Aplicado & $\mathrm{BM}$ & 40 & 46 & 15 & 2 & 6 & 48 \\
\hline- & & BO & 35 & 50 & 0 & 2 & 0 & 54 \\
\hline - & & JO & 35 & 50 & 0 & 2 & 6 & 54 \\
\hline - & Fertilizante & CFS & 56 & 50 & 48 & 0 & 0 & 0 \\
\hline - & กão & $\mathrm{BM}$ & 54 & 44 & 6 & 4 & 4 & 31 \\
\hline - & Aplicado & $\mathrm{BO}$ & 54 & 50 & 0 & 8 & 0 & 46 \\
\hline - & & JO & 54 & 44 & 0 & 8 & 0 & 46 \\
\hline 6 & Fertilizante & CFS & 21 & 60 & 23 & $\overline{0}$ & 0 & 35 \\
\hline - & Aplicado & BM & 2 & 38 & 8 & 73 & 10 & 56 \\
\hline - & & BO & 0 & 38 & 4 & 75 & 10 & 63 \\
\hline 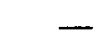 & & JO & 0 & 38 & 4 & 75 & 10 & 63 \\
\hline - & Fertilizante & CFS & 79 & 40 & 31 & 0 & 0 & $\frac{10}{10}$ \\
\hline - & กล̃o & BM & 6 & 29 & 10 & 19 & 23 & 25 \\
\hline - & Aplicado & $\mathrm{BO}$ & 4 & 29 & 4 & 21 & 23 & 29 \\
\hline & & $\mathrm{JO}$ & 4 & 29 & 4 & 21 & 23 & 29 \\
\hline
\end{tabular}

CFS - Critério de Faixas de Suficiência; BO - Beaufils Original; BM - Beaufils Modificado e JO - Jones 
Tabela 21. Índices de avaliação dos métodos para diagnose do estado nutricional em cada local da rede de ensaio de adubação $\mathrm{N}, \mathrm{P}$ e K.

\begin{tabular}{|c|c|c|c|c|c|c|c|c|c|c|c|}
\hline \multirow{2}{*}{\multicolumn{2}{|c|}{ LocalMétodo }} & \multicolumn{3}{|c|}{ Exatidão } & \multicolumn{3}{|c|}{ Razão $\left(V^{+}: F^{+}\right)$} & \multicolumn{4}{|c|}{ Var.Líq.Média da Produtividade } \\
\hline & & $\mathrm{N}$ & $\mathbf{p}$ & $\mathrm{K}$ & TFN & TFP & TFK & $\mathrm{d}\left(\mathrm{Y}_{\mathrm{N}}\right)$ & $\mathrm{d}\left(\mathrm{Y}_{\mathrm{P}}\right)$ & $\mathrm{d}\left(\mathrm{Y}_{\mathrm{K}}\right)$ & $d(Y)$ \\
\hline & & & $-\%$ & & & & & & $-t$ & & \\
\hline \multirow[t]{5}{*}{1} & CFS & 21 & 50 & 23 & 一 & 一 & 一 & $-0,88$ & 0,08 & $-1,05$ & $\longrightarrow$ \\
\hline & $\mathrm{BM}$ & 31 & 50 & 23 & - & - & - & $-0,73$ & $-0,07$ & $-1,05$ & 2,39 \\
\hline & BO & 46 & 50 & 23 & - & - & 一 & 0,14 & $-0,07$ & $-1,05$ & 2,75 \\
\hline & JO & 50 & 50 & 23 & 一 & - & - & 0,36 & $-0,07$ & $-1,05$ & 2,72 \\
\hline & 一 & & - & & - & & - & & - & & \\
\hline \multirow[t]{5}{*}{2} & CFS & 79 & 0 & 92 & 4 & - & 10 & 1,24 & $-3,74$ & 3,00 & 5,09 \\
\hline & $\mathrm{BM}$ & 83 & 0 & 90 & 5 & - & 8 & 2,57 & $-2,38$ & 2,81 & 3,48 \\
\hline & BO & 83 & 0 & 88 & 5 & - & 7 & 2,57 & $-2,38$ & 2,78 & 3,33 \\
\hline & JO & 81 & 0 & 85 & 5 & - & 6 & 2,46 & $-2,38$ & 2,77 & 3,33 \\
\hline & - & & - & & - & & - & & - & & \\
\hline \multirow[t]{5}{*}{3} & CFS & 23 & 25 & 60 & - & - & - & $-0,60$ & $-1,84$ & 0,29 & - \\
\hline & BM & 71 & 33 & 56 & 4 & 2 & 0 & 0,98 & $-1,05$ & 0,34 & 1,22 \\
\hline & BO & 83 & 29 & 50 & 5 & 1 & 0 & 1,07 & $-1,12$ & 0,08 & 1,01 \\
\hline & JO & 94 & 25 & 50 & 18 & 1 & 0 & 1,11 & $-1,11$ & 0,08 & 1,16 \\
\hline & - & & - & & - & & - & & 一 & & \\
\hline \multirow[t]{5}{*}{4} & CFS & 10 & 44 & 52 & - & - & 0 & $-1,85$ & $-0,22$ & $-0,31$ & 0,00 \\
\hline & BM & 58 & 38 & 48 & 7 & 0 & 0 & 1,72 & $-0,24$ & $-0,02$ & 3,01 \\
\hline & $\mathrm{BO}$ & 83 & 38 & 60 & 8 & 0 & 1 & 2,87 & $-0,24$ & 0,20 & 2,46 \\
\hline & JO & 81 & 38 & 58 & 8 & 0 & 1 & 2,82 & $-0,24$ & 0,18 & 2,48 \\
\hline & - & & - & & - & & - & & - & & \\
\hline \multirow[t]{5}{*}{5} & CFS & 44 & 50 & 52 & - & 一 & - & $-0,27$ & $-0,33$ & 0,32 & - \\
\hline & $\mathrm{BM}$ & 42 & 52 & 63 & 1 & 2 & 2 & $-0,29$ & $-0,08$ & 0,18 & 0,15 \\
\hline & BO & 38 & 50 & 54 & 0 & - & 1 & $-0,40$ & $-0,12$ & 0,06 & 0,06 \\
\hline & JO & 38 & 56 & 54 & 0 & - & 1 & $-0,40$ & 0,02 & 0,06 & 0,06 \\
\hline & - & & - & & - & & 一 & & - & & \\
\hline \multirow[t]{4}{*}{6} & CFS & 21 & 60 & 58 & - & - & 3 & $-0,62$ & 0,09 & 0,08 & 1,15 \\
\hline & $\mathrm{BM}$ & 75 & 48 & 65 & 4 & 0 & 2 & 1,13 & $-0,03$ & 0,65 & 1,44 \\
\hline & BO & 75 & 48 & 67 & 4 & 0 & 2 & 1,25 & $-0,03$ & 0,82 & 1,38 \\
\hline & JO & 75 & 48 & 67 & 4 & 0 & 2 & 1,25 & $-0,03$ & 0,82 & 1,38 \\
\hline
\end{tabular}

CFS - Critério de Faixas de Suficiência; BO - Beaufils Original; BM - Beaufils Modificado e JO - Jones 
A descrição do comportamento dos métodos foi similar para $\mathrm{N}$ e $\mathrm{K}$, enquanto que para o $\mathrm{P}$ o desempenho de todos os métodos em avaliação foram semelhantes. As recomendações baseadas em diagnoses bem sucedidas estão apresentadas na Tabela 22, nos quadrantes I e III onde estão os casos em que a diagnose resultou em ganho de produtividade ou evitou adubação desnecessária, respectivamente. Enquanto que as diagnoses mal sucedidas estão no quadrante II, no qual a diagnose não detectou o potencial de resposta da planta, e no quadrante IV, pois a aplicação do adubo foi recomendada e a produtividade permaneceu inalterada ou foi reduzida.

O desempenho do método DRIS foi superior ao CFS independente do procedimento de cálculo, sob as condições em que foi desenvolvido este trabalho. $\mathrm{O}$ CFS apresentou baixa perda de oportunidade no aumento de produtividade, pela não aplicação de fertilizantes em plantas potencialmente produtivas. Este método apresentou um grande percentual de casos de adubação desnecessária. Quanto aos procedimentos baseados no DRIS, o percentual de perda de produtividade foi maior que o CFS, mas a adubação desnecessária foi menor.

Outros parâmetros para avaliar os métodos também propostos por Beverly \& Hallmark (1992), como a exatidão, a razão entre as diagnoses bem ( $\mathrm{V}^{+}$) e mal $\left(\mathrm{F}^{+}\right)$sucedidas; e a resposta em produtividade líquida média, são apresentados na Tabela 23.

Nesta tabela a exatidão para o $\mathrm{N}$ foi superior nos métodos DRIS em relação ao CFS. No entanto, os números são similares para a exatidão da diagnose de $\mathrm{P}$ e K. A razão $\left(\mathrm{V}^{+}: \mathrm{F}^{+}\right)$também seguiu o mesmo comportamento da exatidão. Para a variação líquida média da produtividade quando a correção foi baseada no nutriente isolado obteve-se um ganho real apenas para o nitrogênio, enquanto que para o fósforo e o potássio o comportamento foi de uma menor redução da produtividade para os métodos DRIS. Quando a correção foi feita pela diagnose do nutriente mais limitante, independente do nutriente, os ganhos médios promovidos pela correção feita segundo as diagnoses pelos métodos DRIS foi muito superior ao do CFS.

Apesar das diferenças entre os métodos DRIS não terem sido testadas estatisticamente, pode-se ordenar a eficiência dos métodos pelos totais da exatidão, 
razão $\left(\mathrm{V}^{+}: \mathrm{F}^{+}\right)$e variação líquida da produtividade. A ordem do melhor para o pior método foi Jones (1981), Beaufils (1973) e Beaufils modificado por Elwali \& Gascho (1984).

Tabela 22. Valores percentuais do total de casos em que a diagnose da necessidade de cada um dos três nutrientes (N, P ou K) pelos quatro métodos (CFS, BM, BO e JO) em avaliação resultou em um incremento da produtividade ou que a produtividade permaneceu inalterada ou foi reduzida, em laranjeiras.

\begin{tabular}{|c|c|c|c|c|c|c|c|c|c|}
\hline & \multicolumn{8}{|c|}{ Respasta de produtividade } \\
\hline & & \multicolumn{4}{|c|}{$\begin{array}{l}\text { Produtividade inalterada ou } \\
\text { reduzida }\end{array}$} & \multicolumn{4}{|c|}{ Produtividade inerementada } \\
\hline \multirow{10}{*}{ 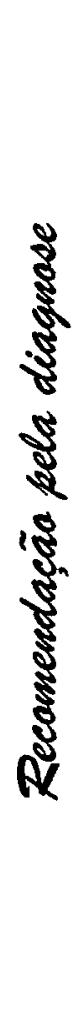 } & \multirow{5}{*}{ 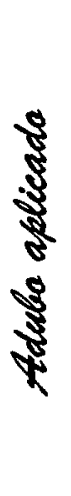 } & $I V$ & $N$ & $\boldsymbol{P}$ & $\boldsymbol{K}$ & $I$ & $N$ & $\boldsymbol{P}$ & $\boldsymbol{K}$ \\
\hline & & $C F S$ & 0 & 0 & 3 & CFS & 0 & 0 & 8 \\
\hline & & $\boldsymbol{B} M$ & 10 & 8 & 14 & $\boldsymbol{B} M$ & 41 & 6 & 21 \\
\hline & & BO & 11 & 8 & 22 & BO & 52 & 5 & 29 \\
\hline & & JO & 9 & 9 & 22 & JO & 53 & 7 & 29 \\
\hline & \multirow{5}{*}{ 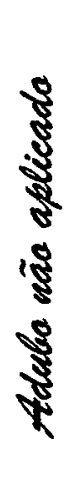 } & $\boldsymbol{I I I}$ & $N$ & $\boldsymbol{P}$ & $\boldsymbol{K}$ & $I I$ & $\boldsymbol{N}$ & $\boldsymbol{P}$ & $\boldsymbol{K}$ \\
\hline & & CFS & 24 & 46 & 41 & CFS & 76 & 54 & 48 \\
\hline & & $\boldsymbol{B M}$ & 14 & 38 & 30 & $\boldsymbol{B M}$ & 35 & 48 & 35 \\
\hline & & $\boldsymbol{B O}$ & 13 & 38 & 22 & BO & 24 & 49 & 28 \\
\hline & & Jo & 15 & 37 & 22 & Jo & 24 & 48 & 28 \\
\hline
\end{tabular}

CFS - Critério de Faixas de Suficiência; BO - Beaufils Original; BM - Beaufils Modificado e JO - Jones

Do total de 320 parcelas experimentais da rede de ensaio de adubação N, P e K somente 240 parcelas foram passíveis de avaliação da diagnose e recomendação. 
Tabela 23. Desempenho geral dos métodos para diagnose do estado nutricional na rede de ensaios de adubação N, P e K, em laranjeiras.

\begin{tabular}{|c|c|c|c|c|c|c|c|c|c|}
\hline \multirow{2}{*}{$\begin{array}{c}\text { Método } \\
-\end{array}$} & \multicolumn{3}{|c|}{ Exatidão } & \multicolumn{3}{|c|}{$\operatorname{Razão}\left(\mathrm{V}^{+}: \mathrm{F}^{+}\right)$} & \multicolumn{3}{|c|}{ Var.Líq.Média Produtividade } \\
\hline & $\mathrm{N}$ & $\mathbf{P}$ & $\mathrm{K}$ & $\overline{\mathrm{TF}_{\mathrm{N}}}$ & $\mathrm{TF}_{\mathrm{P}}$ & $\mathrm{TF}_{\mathrm{K}}$ & $d\left(Y_{N}\right) d\left(Y_{P}\right) d\left(Y_{K}\right)$ & $d(Y)$ & n.c. \\
\hline & & $-\%$ & - & & & & $\mathrm{t} \mathrm{ha}^{-1}$ & & \\
\hline CFS & 24 & 46 & 49 & 0 & 0 & 1 & $-0,84-0,45-0,13$ & 0,23 & 9 \\
\hline $\mathrm{BO}$ & 65 & 43 & 51 & 3 & 0 & 1 & $0,99-0,32 \quad 0,02$ & 0,27 & 226 \\
\hline BM & 55 & 44 & 51 & 3 & 1 & 1 & $0,56-0,29 \quad 0,02$ & 0,29 & 181 \\
\hline $\mathrm{JO}$ & 68 & 43 & 50 & 6 & 0 & 1 & $1,03-0,28 \quad 0,02$ & 0,28 & 223 \\
\hline
\end{tabular}

CFS - Critério de Faixas de Suficiência; BO - Beaufils Original; BM - Beaufils Modificado e JO - Jones n.c. número de correções de cada método de um total de 320 parcelas experimentais submetidas a diagnose. 


\section{CONCLUSÕES}

A população referência teve papel fundamental na diagnose do estado nutricional através do DRIS, já que o método é baseado em comparações.

$\mathrm{Na}$ definição do procedimento padrão os índices-DRIS diferiram em grandeza entre um procedimento e outro mas as diagnoses apresentaram eficiências semelhantes.

Os métodos apresentaram:

- maior realce para deficiência, no caso do proposto por Beaufils (1973),

- menor número de interpretações errôneas, contudo considerou desnecessária a correção pela adubação N, P e K para plantas com potencial de incremento da produtividade, no caso do procedimento Beaufils Modificado (Elwali \& Gascho, 1984), e

- maior facilidade de cálculo, além de maior formalidade estatística, no caso por Jones (1981).

- baixa sensibilidade na identificação de plantas com desequilíbrios nutricionais no Critério de Faixas de Suficiência

O DRIS, independente do procedimento de cálculo, apresentou melhor desempenho que o método tradicional, o Critério de Faixas de Suficiência, na orientação de correções de desequilíbrios nutricionais na cultura dos citros, principalmente para a diagnose dos teores foliares do nitrogênio.

Pelo desempenho numericamente melhor e facilidade de cálculo o procedimento proposto por Jones (1981) foi escolhido como o procedimento padrão para o cálculo dos índices-DRIS. 


\section{REFERÊNCIAS BIBLIOGRÁFICAS}

AMUNDSON, R.L. \& KOEHLER, F.E. Utilization of DRIS for diagnosis of nutrient deficiences in winter wheat. Agron. J., v.79, p.472-476, 1987.

ANDRADE, D.F.; NOLETO, A.Q. Exemplos de fatoriais fracionados (1/2) $4^{3}$ e (1/4) $4^{4}$ para ajuste de modelos polinomiais quadráticos. Pesq. Agropec. bras., v.21, n.6, p.677-680, Jun. 1986.

BATAGLIA, O.C.; DECHEN, A.R. Critérios alternativos para diagnose foliar. In: SIMPÓSIO AVANÇADO DE QUÍMICA E FERTILIDADE DO SOLO, Piracicaba, 1986. Anais. Campinas: Fundação Cargill, 1986. p.115-136.

BATAGLIA, O.C.; SANTOS, W.R. Efeito do procedimento de cálculo e da população e referência nos índices do Sistema Integrado de Diagnose e Recomendação (DRIS). R. Bras. Ci. Solo, v.14, p.339-344, 1990.

BATAGLIA, O.C.; DECHEN, A.R.; SANTOS, W.R. Diagnose visual e análise de plantas. In: DECHEN, A.R.; BOARETTO, A.E.; VERDADE, F.C. (Ed.) Adubação, Produtividade e Ecologia. Campinas: Fundação Cargill, 1992. p.369-393.

BATAGLIA, O.C.; DECHEN, A.R.; SANTOS, W.R. Princípios da diagnose foliar. In: ALVAREZ, V.H.; FONTES, L.E.F.; FONTES, M.P.F. (Ed.) O solo nos grandes domínios morfoclimáticos do Brasil e o desenvolvimento sustentado. Viçosa, MG: SBCS; UFV, DPS, 1996. p.647-660.

BATAGLIA, O.C.; FURLANI, A.M.C.; TEIXEIRA, J.P.F.; FURLANI, P.R.; GALLO, J.R. Métodos de análise química de plantas. Campinas: Instituto Agronômico, 1983. 48p. (Boletim Técnico, 78) 
BEAUFILS, E.R. Physiological diagnosis - a guide for improving maize production based on principles developed for rubber trees. Fertilizer Society of South African Journal, v.1, p.1-30, 1971.

BEAUFILS, E.R. Diagnosis and recommendation integrated system (DRIS). Pietermaritzburg, S.Africa: University of Natal, 1973. 132p. (Soil Sci. Bull., n.1)

BEVERLY, R.B. Prescient diagnostic analysis shows sufficiency ranges approach superior to DRIS for citrus. Commun. in Soil Science and Plant Analysis, v.23, n.17-20, p.2641-2649, 1992.

BEVERLY, R.B.; HALLMARK, W.B. Prescient diagnostic analysis: a proposed new approach to evaluating plant nutrient disgnostic methods. Commun. in Soil Science and Plant Analysis, v.23, n.17-20, p.2633-2640, 1992.

BEVERLY, R.B.; STARK, J.C.; OJALA, J.C.; EMBLETON, T.W. Nutrient diagnosis of "Valencia" oranges by DRIS. J.Amer. Soc. Hort. Sci. V.109, p.649-654, 1984.

CANTARELLA, H.; QUAGGIO, J.A., BATAGLIA, O.C.; van RAIJ, B. Response of citrus to NPK fertilization in a network of field trials in São Paulo State, Brasil. In: INTERNATIONAL CITRUS CONGRESS, VII, Acireale,Italy, 1992. Proceedings of the International Society of Citriculture. Trento, Italy: International Society of Citriculture, 1994. v.2, p.607-612.

CHAPMAN, H.D. Diagnostic criteria for plants and soils. Riverside: Univ.of California, 1966. 793p.

COLWELL, J.D. Computations for studies of soil fertility and fertilizer requirements. Commonw. Agric. Bur., 1978. 297p. 
COSTA, A.N.da Uso do sistema integrado de diagnose e recomendacao (DRIS) na avaliação do estado nutricional do mamoeiro (Carica papaya L.) no Estado do Espírito Santo. Viçosa, 1995. 95p. Tese (Doutorado) - Universidade Federal de Viçosa.

COTTENIE, A. Present status of plant analysis as a method for preparation of fertilizer recommendations. In: FAO. Soils. Rome: FAO, 1980. p.21-36. (FAO Bulletin, 38/1)

CRESTE, J.E. Uso do DRIS na avaliação do estado nutricional do limoeiro siciliano. Botucatu, 1996. 120p. Tese (Doutorado) - Faculdade de Ciências Agrárias e Veterinárias, Universidade Estadual Paulista "Julio de Mesquita Filho".

ELWALI, A.M.O.; GASCHO, G.J. Soil testing, foliar analysis, and DRIS as guide for sugarcane fertilization. Agron. J., v.76, p.466-470, 1984.

ELWALI, A.M.O.; GASCHO, G.J.; SUMNER, M.E. Sufficiency levels and DRIS norms for 11 nutrients in corn. Agron. J., v.77, p.506-508, 1985.

GALLO, J.R.; COELHO, F.A.S.; MIRANDA, L.T. A análise foliar na nutrição do milho. I - Resultados preliminares. Bragantia, v.24, p.XLVII-LIII, 1965. (Nota n.9).

GALLO, J.R.; RODRIGUEZ, O.; CAMARGO, A.P.; IGUE, T. Variações anuais na produção de frutos e concentração de macronutrientes em folhas de citros, relacionadas com o balanço hídrico metereológico e adubação no período de 1957 a 1975. Bragantia, v.36, p.271-289, 1977.

GERGOLETTI, I.F. Avaliação do estado nutricional da videira - cultivar itália em três estádios de desenvolvimento em São Miguel Arcanjo/SP, utilizando o método DRIS. Piracicaba, 1995. 84p. Dissertação (Mestrado) - Escola Superior de Agricultura "Luiz de Queiroz", Universidade de São Paulo. 
GRUPO PAULISTA DE ADUBAÇÃO E CALAGEM PARA CITROS. Recomendações de adubação e calagem para citros no Estado de São Paulo. Cordeirópolis: Laranja Revista Técnico-Científica de Citricultura, 1994. 27p. (Edição especial).

GUILHERME, M.A.S. Avaliação do estado nutricional da videira itália em três estádios de desenvolvimento, na região de Jales/SP, utilizando o método DRIS. Piracicaba, 1995. 81 p. Dissertação (Mestrado) - Escola Superior de Agricultura "Luiz de Queiroz", Universidade de São Paulo.

HALLMARK, W.B.; deMOOY, C.J.; PESEK, J. Comparison of two DRIS methods for diagnosing nutrient deficiences. Journal of Fertilizer Issues, v.4, n.4, p.151-158, Dec.1987.

HALLMARK, W.B.; WALWORTH, J.L.; SUMNER, M.E.; deMOOY, C.J.; PESEK, J.; SHAO, K.P. Separating limitting from non-limiting nutrients. Journal of Plant Nutrition, v.10,n.9-16, p.1381-1390, 1987.

HIROCE, R. Efeito de variedades e de porta-enxerto na composição mineral das folhas de citros. Laranja: Revista Técnico-Científica de Citricultura, v.8, n.1, p.239$282,1987$.

JONES, C.A. Proposed modifications of the Diagnosis and Recommendation Integrated System (DRIS) for interpreting plant analyses. Commun. in Soil Science and Plant Analysis, v.12, p.785-794, 1981.

JONES, M.B. Sulfur availability index. In: Sulfur in agriculture. Madison: Am. Soc. Agronomy, Agronomy Monograph n.27, 1986. P.549-566.

JONES, J.B.; WOLF, B.; MILLS, H.A. Plant analysis handbook: a practical sampling, preparation, analysis, and interpretation guide. MICRO-MACRO Publ. 1991. 213p. 
LEANDRO, W.M.; OLIVEIRA, S.A.; BRASIL, E.M.; OLIVEIRA JUNIOR, J.P.; CHITOLINA, J.C.; REIS, R.T.; ARTIAGA, S.R.; PEREIRA, S.R.; BOTELHO, S.A. Níveis de suficiência no solo para soja (BR-15 e IAC-15) em Goianésia/GO, através do método DRIS. In: SIMPÓSIO SOBRE O CERRADO, 8.; INTERNATIONAL SYMPOSIUM ON TROPICAL SAVANNAS, 1., Brasília, 1996. Anais. Planaltina : Embrapa-Cpac, 1996. P.337-40.

LEE, P.F.W. Initial observations on the application of DRIS aproach to tea (Camellia sinensis L.). Foliar analysis results in South Africa. Potash Review, Subject 27, n.7 p.1-6, 1981 .

LETZSCH, W.S. Computer program for selection of norms for use in the diagnosis and recommendation integrated system (DRIS). Commun. in Soil Science and Plant Analysis, v.16, n.4, p.339-347, 1985.

LOURENÇO FILHO, R.C.B. Controle estatístico de qualidade. Rio de Janeiro: LTC Livros Técnicos e Científicos Editora S.A.,1982. cap.2, p.35-47: Gráficos de contrôle.

MALAVOLTA, E.; MALAVOLTA, M.L. Diagnose Foliar - Princípios e aplicações. In: SIMPÓSIO SOBRE INTERPRETAÇÃO DE ANÁLISE QUÍMICA DE SOLO E PLANTA PARA FINS DE ADUBAÇÃO, Botucatu, 1988. Anais. Botucatu: UNESP, 1988. 86p.

MALAVOLTA, E.; VITTI, G.C.; OLIVEIRA, S.A. Avaliação do estado nutricional das plantas: princípios e aplicações. Piracicaba: Associação Brasileira para Pesquisa da Potassa e do Fosfato, 1989. 201p.

MARSCHNER, H. Functions of Mineral Nutrients: Macronutrients. In: Mineral nutrition of higer plants. London: Academic Press Inc., 1986. p.195-267.

MENGEL, K. \& KIRKBY, E.A. Principles of Plant Nutrition. Bern, Switzerland: International Potash Institute, 593p. 1978. 
MUNSON, R.D.; NELSON, W.L. Principles and practices in plant analysis. In: WALSH, L.M.; BEATON, J.D. (Ed.) Soil testing and plant analysis, 1973. P.223248.

OKHI, K. Critical nutrient levels related to plant growth and some physiological process. Journal of Plant Nutrition, v.10, p.1583-1590, 1987.

OLIVEIRA, R.F.; CRUZ, E.S.; BASTOS, J.B.; ALBUQUERQUE, F.C.; MURAOKA, T. \& SASAKI, G.K. Avaliação do estado nutricional de pimenta-do-reino (Piper nigrum) pelo DRIS, em duas épocas, em Tomé-Açú, PA. In: Reunião Brasileira de Fertilidade do Solo e Nutrição de Plantas, 22., Manaus, 1996. Resumos. Manaus: Ed. Universidade do Amazonas, 1996. p.26-7.

QUAGGIO, J.A. Respostas da laranjeira Valência (Citrus sinnensis L.Osbeck) sobre limoeiro Cravo (Citrus limonia (L.) Burmann) à calagem e ao equilibrio de bases num latossolo vermelho escuro de textura argilosa. Piracicaba, 1991. 105p. Tese (Doutorado) - Escola Superior de Agronomia "Luiz de Queiroz", Universidade de São Paulo.

SALISBURY, F.B.; ROSS, C.W. Mineral nutrition. In: SALISBURY, F.B.; ROSS, C.W. Plant physiology. Belmont: Wadsworth Publishing Co., 1991. cap.6, p.116135.

STEEL, R.G.D.; TORRIE, J.H. Principles and procedures of statistics, with special reference to biological sciences. New York: McGraw Hill Book Company Inc., 1960. 481p.

SUMNER, M.E. Use of DRIS system in foliar diagnosis of crop at high yield levels. Commun. in Soil Science and Plant Analysis, v.8, p.251-268, 1977.

TAIZ, L.; ZAIGER, E. Mineral nutrition. In: TAIZ, L.; ZAIGER, E. Plant physiology. Redwood City: The Benjamin/Cummings Publishing Company Inc., 1991. cap.5, p.100-119. 
TERBLANCHE, J.H.; DU PLESIS, S.F. Summary of workshp on leaf and soil analysis as a tool for determining fertilizer requirements of citrus. Proc. Int. Soc. Citriculture, v.2, p.744-745, 1992.

ULRICH, A.; HILLS, F.J. Principles and practices of plant analysis. In: Soil testing and plant analysis. Madison: SSSA, 1967. Pt.2, p.11-24.

WADT, P.G.S. \& GONÇALVES, J.L.M. Relacionamento entre classe de solo e o estado nutricional de cafeeiros determinado pelos métodos do nível crítico e do DRIS. In: Congresso Brasileiro de Ciência do Solo, 26, Rio de Janeiro, 1997. Resumos. Rio de Janeiro: SBCS, 1997. p.133.

WALWORTH, J.L.; SUMNER, M.E.; ISAAC, R.A.; PLANCK, C.O. Preliminary DRIS norms for alfafa in the Southeastern United State and a composition with midwestern norms. Agron. J., v.78, p.1046-1052, 1986.

WEIR, C.C. Nutrient element balance in citrus nutrition. Plant and Soil XXX, n.3, p.405-414. Jun. 1969.

WOODS, D.B.; VILLIERS, J.M. Diagnosing the nutrient status of "Valencia" oranges in Southern Africa. Proc. Int. Soc. Citriculture, v.2, p.556-559, 1992.

ZAMBELLO Jr., E. Aplicação do Sistema Integrado de Diagnose e Recomendação para diferentes solos e épocas de amostragem foliar em soqueiras de cana-de-açúcar (Saccharum spp). Piracicaba, 1979. 95p. Dissertação (M.S.) - Escola Superior de Agricultura "Luiz de Queiroz", Universidade de São Paulo. 
APÊNDICE 
Apêndice 1. Parâmetros estatísticos da população de referência para citros com produtividade média de 38,01 tha e população de baixa produtividade (33,86 t/ha) em Araraquara/SP.

\begin{tabular}{|c|c|c|c|c|c|c|c|c|c|c|c|}
\hline \multirow{3}{*}{ Relação } & \multicolumn{10}{|c|}{ População } & \multirow{3}{*}{$e_{\text {ated }}^{2} / e_{\text {batia }}^{2}$} \\
\hline & \multicolumn{5}{|c|}{ Alta produtividade } & \multicolumn{5}{|c|}{ Baixa produtividade } & \\
\hline & $x$ & $e^{2}$ & $c$ & c.v. & $n$ & $x$ & $e^{2}$ & 4 & c.v. & $n$. & \\
\hline$\overline{\mathrm{N} / \mathrm{P}}$ & 19,8008 & 0,9185 & 0,9583 & 0,0484 & 37 & 19,0297 & 1,7076 & 1,3067 & 0,0686 & 27 & 0,5378 \\
\hline $\mathrm{N} / \mathrm{K}$ & 1,8008 & 0,0195 & 0,1396 & 0,0775 & 37 & 1,7985 & 0,0333 & 0,1827 & 0,1016 & 27 & 0,5843 \\
\hline $\mathrm{N} / \mathrm{Ca}$ & 0,8114 & 0,0006 & 0,0255 & 0,0314 & 37 & 0,8021 & 0,0002 & 0,0141 & 0,0176 & 27 & 3,2500 \\
\hline $\mathrm{N} / \mathrm{Mg}$ & 11,0240 & 0,7676 & 0,8761 & 0,0794 & 37 & 10,9704 & 1,7075 & 1,3067 & 0,1191 & 27 & 0,4495 \\
\hline$N / S$ & 10,4684 & 0,0812 & 0,2850 & 0,0272 & 37 & 9,7216 & 0,4015 & 0,6336 & 0,0651 & 27 & 0,2024 \\
\hline $\mathrm{P} / \mathrm{N}$ & 0,0506 & 0,0001 & 0,0025 & 0,0495 & 37 & 0,0527 & 0,0001 & 0,0035 & 0,0676 & 27 & 1,0000 \\
\hline $\mathrm{P} / \mathrm{K}$ & 0,0910 & 0,0001 & 0,0068 & 0,0753 & 37 & 0,0945 & 0,0001 & 0,0072 & 0,0763 & 27 & 1,0000 \\
\hline $\mathrm{P} / \mathrm{Ca}$ & 0,0411 & 0,0001 & 0,0029 & 0,0721 & 37 & 0,0423 & 0,0001 & 0,0031 & 0,0746 & 27 & 1,0000 \\
\hline $\mathrm{P} / \mathrm{Mg}$ & 0,5573 & 0,0018 & 0,0430 & 0,0771 & 37 & 0,5794 & 0,0070 & 0,0841 & 0,1452 & 27 & 0,2613 \\
\hline $\mathrm{P} / \mathrm{S}$ & 0,5295 & 0,0004 & 0,0206 & 0,0390 & 37 & 0,5114 & 0,0004 & 0,0222 & 0,0434 & 27 & 0,8775 \\
\hline $\mathbf{K} / \mathbf{N}$ & 0,5585 & 0,0018 & 0,0433 & 0,0775 & 37 & 0,5613 & 0,0030 & 0,0551 & 0,0983 & 27 & 0,6163 \\
\hline $\mathbf{K} / \mathbf{P}$ & 11,0445 & 0,6649 & 0,8154 & 0,0738 & 37 & 10,6364 & 0,6006 & 0,7750 & 0,0728 & 27 & 1,1071 \\
\hline $\mathrm{K} / \mathrm{Ca}$ & 0,4538 & 0,0019 & 0,0446 & 0,0983 & 37 & 0,4503 & 0,0020 & 0,0452 & 0,1004 & 27 & 0,9707 \\
\hline $\mathrm{K} / \mathbf{M g}$ & 6,1776 & 0,7356 & 0,8577 & 0,1388 & 37 & 6,1944 & 1,4849 & 1,2185 & 0,1967 & 27 & 0,4954 \\
\hline $\mathrm{K} / \mathrm{S}$ & 5,8440 & 0,1918 & 0,4379 & 0,0749 & 37 & 5,4338 & 0,1509 & 0,3885 & 0,0715 & 27 & 1,2709 \\
\hline $\mathrm{Ca} / \mathrm{N}$ & 1,2335 & 0,0014 & 0,0383 & 0,0310 & 37 & 1,2470 & 0,0004 & 0,0220 & 0,0177 & 27 & $3,0000 * *$ \\
\hline $\mathrm{Ca} / \mathrm{P}$ & 24,4447 & 2,9663 & 1,7223 & 0,0704 & 37 & 23,7386 & 3,2483 & 1,8023 & 0,0759 & 27 & 0,9132 \\
\hline $\mathrm{Ca} / \mathrm{K}$ & 2,2242 & 0,0480 & 0,2192 & 0,0985 & 37 & 2,2425 & 0,0517 & 0,2274 & 0,1014 & 27 & 0,9290 \\
\hline $\mathrm{Ca} / \mathrm{Mg}$ & 13,5974 & 1,2943 & 1,1377 & 0,0836 & 37 & 13,6741 & 2,4709 & 1,5719 & 0,1149 & 27 & 0,5238 \\
\hline $\mathrm{Ca} / \mathrm{S}$ & 12,9127 & 0,2767 & 0,5260 & 0,0407 & 37 & 12,1217 & 0,6371 & 0,7982 & 0,0658 & 27 & 0,4343 \\
\hline $\mathrm{Mg} / \mathrm{N}$ & 0,0912 & 0,0001 & 0,0070 & 0,0770 & 37 & 0,0922 & 0,0001 & 0,0100 & 0,1085 & 27 & 0,5000 \\
\hline $\mathrm{Mg} / \mathrm{P}$ & 1,8045 & 0,0187 & 0,1367 & 0,0757 & 37 & 1,7568 & 0,0502 & 0,2240 & 0,1275 & 27 & 0,3727 \\
\hline $\mathrm{Mg} / \mathrm{K}$ & 0,1648 & 0,0005 & 0,0222 & 0,1351 & 37 & 0,1667 & 0,0008 & 0,0283 & 0,1698 & 27 & 0,6250 \\
\hline $\mathrm{Mg} / \mathrm{Ca}$ & 0,0740 & 0,0001 & 0,0062 & 0,0843 & 37 & 0,0740 & 0,0001 & 0,0080 & 0,1085 & 27 & 0,6666 \\
\hline $\mathrm{Mg} / \mathrm{S}$ & 0,9549 & 0,0053 & 0,0728 & 0,0763 & 37 & 0,8969 & 0,0117 & 0,1085 & 0,1209 & 27 & 0,4507 \\
\hline $\mathbf{S} / \mathbf{N}$ & 0,0956 & 0,0001 & 0,0026 & 0,0275 & 37 & 0,1032 & 0,0001 & 0,0066 & 0,0644 & 27 & 0,2500 \\
\hline $\mathbf{S} / \mathbf{P}$ & 1,8914 & 0,0055 & 0,0746 & 0,0394 & 37 & 1,9589 & 0,0078 & 0,0885 & 0,0451 & 27 & 0,7113 \\
\hline $\mathbf{S} / \mathbf{K}$ & 0,1720 & 0,0001 & 0,0130 & 0,0755 & 37 & 0,1849 & 0,0001 & 0,0129 & 0,0700 & 27 & 1,0000 \\
\hline $\mathrm{S} / \mathrm{Ca}$ & 0,0775 & 0,0001 & 0,0032 & 0,0416 & 37 & 0,0828 & 0,0001 & 0,0053 & 0,0649 & 27 & 0,3333 \\
\hline $\mathrm{S} / \mathrm{Mg}$ & 1,0534 & 0,0071 & 0,0845 & 0,0802 & 37 & 1,1331 & 0,0250 & 0,1581 & 0,1395 & 27 & 0,2858 \\
\hline$N$ & 26,4400 & 0,0031 & 0,5560 & 0,0210 & 37 & 25,7220 & 0,0066 & 0,8130 & 0,0316 & 27 & 0,4689 \\
\hline $\mathbf{P}$ & 1,3370 & 0,0000 & 0,0590 & 0,0444 & 37 & 1,3550 & 0,0001 & 0,0640 & 0,0472 & 27 & 1,0000 \\
\hline K & 14,7510 & 0,0087 & 0,9320 & 0,0632 & 37 & 14,4030 & 0,0116 & 1,0760 & 0,0747 & 27 & 0,7500 \\
\hline $\mathrm{Ca}$ & 32,6210 & 0,0193 & 1,3900 & 0,0426 & 37 & 32,0740 & 0,0120 & 1,0990 & 0,0342 & 27 & 1,6009 \\
\hline $\mathbf{M g}$ & 2,4130 & 0,0004 & 0,2010 & 0,0835 & 37 & 2,3740 & 0,0070 & 0,2650 & 0,1118 & 27 & 0,5857 \\
\hline $\mathbf{S}$ & 2,5270 & 0,0001 & 0,0690 & 0,0274 & 37 & 2,6500 & 10,0010 & 0,1010 & 0,0382 & 27 & 0,5000 \\
\hline
\end{tabular}

$x:$ média; $4^{2}$ : variância: $₫$ : desvio padrão; c.v.: coeficiente de variação; $n .:$ número de observações;

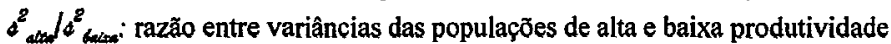

Os valores de $\mathrm{F}$ nos níveis de 5 e $1 \%$ de significância são 1,47 e 1,73, respectivamente (Steel \& Torrie, 1960). 
Apêndice 2. Parâmetros estatísticos da população de referência para citros com produtividade média de 55,82 tha e população de baixa produtividade ( 46,16 t/ha) em Botucatu/SP.

\begin{tabular}{|c|c|c|c|c|c|c|c|c|c|c|c|}
\hline \multirow{3}{*}{ Relação } & \multicolumn{10}{|c|}{ População } & \multirow{3}{*}{$e_{\text {attat }}^{2} / d_{\text {basar }}^{2}$} \\
\hline & \multicolumn{5}{|c|}{ Alta produtividade } & \multicolumn{5}{|c|}{ Baixa produtividade } & \\
\hline & $x$ & $d^{2}$ & 4 & c.v. & $n$ & $x$ & $d^{2}$ & $c$ & c.v. & $n$. & \\
\hline $\bar{N} / P$ & 14,8862 & 0,9859 & 0,9929 & 0,0667 & 34 & 14,9205 & 1,8394 & 1,3562 & 0,0909 & 30 & 0,5360 \\
\hline $\mathrm{N} / \mathrm{K}$ & 3,0825 & 2,6327 & 1,6225 & 0,5263 & 34 & 4,0329 & 5,3152 & 2,3054 & 0,5716 & 30 & 0,4953 \\
\hline $\mathrm{N} / \mathrm{Ca}$ & 0,5710 & 0,0084 & 0,0918 & 0,1608 & 34 & 0,5061 & 0,0081 & 0,0900 & 0,1778 & 30 & 1,0419 \\
\hline $\mathrm{N} / \mathrm{Mg}$ & 4,1775 & 0,2439 & 0,4939 & 0,1182 & 34 & 3,7184 & 0,4708 & 0,6861 & 0,1845 & 30 & 0,5182 \\
\hline $\mathrm{N} / \mathrm{S}$ & 10,1405 & 3,7862 & 1,9458 & 0,1918 & 34 & 8,6709 & 3,6272 & 1,9045 & 0,2196 & 30 & 1,0438 \\
\hline $\mathrm{P} / \mathrm{N}$ & 0,0674 & 0,0000 & 0,0042 & 0,0636 & 34 & 0,0675 & 0,0000 & 0,0058 & 0,0869 & 30 & 0,6666 \\
\hline $\mathrm{P} / \mathrm{K}$ & 0,2101 & 0,0140 & 0,1184 & 0,5637 & 34 & 0,2683 & 0,0224 & 0,1498 & 0,5583 & 30 & 0,6253 \\
\hline $\mathrm{P} / \mathrm{Ca}$ & 0,0382 & 0,0000 & 0,0048 & 0,1255 & 34 & 0,0339 & 0,0000 & 0,0055 & 0,1626 & 30 & 0,6666 \\
\hline $\mathrm{P} / \mathrm{Mg}$ & 0,2808 & 0,0009 & 0,0299 & 0,1067 & 34 & 0,2503 & 0,0022 & 0,0476 & 0,1902 & 30 & 0,3964 \\
\hline $\mathrm{P} / \mathrm{S}$ & 0,6845 & 0,0214 & 0,1463 & 0,2138 & 34 & 0,5773 & 0,0085 & 0,0925 & 0,1603 & 30 & $2,5005 * *$ \\
\hline $\mathrm{K} / \mathrm{N}$ & 0,3862 & 0,0194 & 0,1393 & 0,3609 & 34 & 0,3708 & 0,0622 & 0,2495 & 0,6728 & 30 & 0,3120 \\
\hline $\mathrm{K} / \mathrm{P}$ & 5,7842 & 4,7081 & 2,1698 & 0,3751 & 34 & 5,4358 & 12,5169 & 3,5379 & 0,6508 & 30 & 0,3761 \\
\hline $\mathrm{K} / \mathrm{Ca}$ & 0,2279 & 0,0104 & 0,1022 & 0,4484 & 34 & 0,2019 & 0,0281 & 0,1678 & 0,8315 & 30 & 0,3707 \\
\hline $\mathrm{K} / \mathrm{Mg}$ & 1,6660 & 0,5248 & 0,7244 & 0,4348 & 34 & 1,5197 & 1,6738 & 1,2937 & 0,8513 & 30 & 0,3135 \\
\hline $\mathrm{K} / \mathrm{S}$ & 3,7383 & 1,0552 & 1,0272 & 0,2748 & 34 & 2,9229 & 2,5312 & 1,5909 & 0,5443 & 30 & 0,4169 \\
\hline $\mathrm{Ca} / \mathrm{N}$ & 1,7946 & 0,0789 & 0,2810 & 0,1566 & 34 & 2,0289 & 0,1001 & 0,3164 & 0,1559 & 30 & 0,7888 \\
\hline $\mathrm{Ca} / \mathrm{P}$ & 26,5352 & 10,6443 & 3,2625 & 0,1229 & 34 & 30,0709 & 16,2769 & 4,0344 & 0,1341 & 30 & 0,6539 \\
\hline $\mathrm{Ca} / \mathrm{K}$ & 5,8208 & 15,8866 & 3,9858 & 0,6847 & 34 & 8,5216 & 27,5955 & 5,2531 & 0,6164 & 30 & 0,5757 \\
\hline $\mathrm{Ca} / \mathrm{Mg}$ & 7,3715 & 0,2373 & 0,4871 & 0,0660 & 34 & 7,3491 & 0,1848 & 0,4299 & 0,0585 & 30 & 1,2837 \\
\hline $\mathrm{Ca} / \mathrm{S}$ & 18,2150 & 22,4835 & 4,7416 & 0,2603 & 34 & 17,5455 & 18,9846 & 4,3571 & 0,2483 & 30 & 1,1843 \\
\hline $\mathrm{Mg} / \mathrm{N}$ & 0,2428 & 0,0009 & 0,0300 & 0,1238 & 34 & 0,2769 & 0,0020 & 0,0453 & 0,1638 & 30 & 0,4368 \\
\hline $\mathrm{Mg} / \mathrm{P}$ & 3,6009 & 0,1495 & 0,3867 & 0,1073 & 34 & 4,1178 & 0,4651 & 0,6820 & 0,1656 & 30 & 0,3215 \\
\hline $\mathrm{Mg} / \mathrm{K}$ & 0,7871 & 0,2897 & 0,5383 & 0,6838 & 34 & 1,1890 & 0,5824 & 0,7632 & 0,6418 & 30 & 0,4975 \\
\hline $\mathrm{Mg} / \mathrm{Ca}$ & 0,1362 & 0,0000 & 0,0097 & 0,0714 & 34 & 0,1365 & 0,0000 & 0,0080 & 0,0587 & 30 & 1,5000 \\
\hline $\mathrm{Mg} / \mathrm{S}$ & 2,4745 & 0,4019 & 0,6340 & 0,2562 & 34 & 2,4181 & 0,4981 & 0,7057 & 0,2918 & 30 & 0,8069 \\
\hline $\mathrm{S} / \mathrm{N}$ & 0,1021 & 0,0003 & 0,0193 & 0,1892 & 34 & 0,1203 & 0,0005 & 0,0239 & 0,1991 & 30 & 0,6491 \\
\hline $\mathbf{S} / \mathbf{P}$ & 1,5194 & 0,0839 & 0,2897 & 0,1906 & 34 & 1,7706 & 0,0641 & 0,2533 & 0,1430 & 30 & 1,3080 \\
\hline $\mathbf{S} / \mathbf{K}$ & 0,2991 & 0,0171 & 0,1308 & 0,4372 & 34 & 0,4466 & 0,0458 & 0,2140 & 0,4792 & 30 & 0,3733 \\
\hline $\mathrm{S} / \mathrm{Ca}$ & 0,0582 & 0,0001 & 0,0138 & 0,2369 & 34 & 0,0609 & 0,0002 & 0,0169 & 0,2787 & 30 & 0,6551 \\
\hline $\mathrm{S} / \mathrm{Mg}$ & 0,4283 & 0,0102 & 0,1011 & 0,2360 & 34 & 0,4516 & 0,0200 & 0,1417 & 0,3138 & 30 & 0,5087 \\
\hline $\mathbf{N}$ & 21,1700 & 1,5300 & 1,2380 & 0,0584 & 34 & 19,6960 & 2,9200 & 1,7100 & 0,0868 & 30 & 0,5237 \\
\hline $\mathbf{P}$ & 1,4260 & 0,0100 & 0,1050 & 0,0738 & 34 & 1,3230 & 0,0001 & 0,0890 & 0,0678 & 30 & 1,3750 \\
\hline K & 8,1320 & 8,0400 & 2,8360 & 0,3487 & 34 & 7,0060 & 18,8900 & 4,3460 & 0,6203 & 30 & 0,4258 \\
\hline $\mathrm{Ca}$ & 37,8140 & 27,2200 & 5,2170 & 0,1379 & 34 & 39,9600 & 48,5900 & 6,9710 & 0,1744 & 30 & 0,5601 \\
\hline Mg & 5,1290 & 0,3800 & 0,6220 & 0,1212 & 34 & 5,4760 & 1,2300 & 1,1110 & 0,2029 & 30 & 0,3133 \\
\hline $\mathbf{S}$ & 2,1440 & 0,0900 & 0,3110 & 0,1453 & 34 & 2,3330 & 0,0900 & 0,3030 & 0,1299 & 30 & 1,0543 \\
\hline
\end{tabular}

$x$ : média; $\AA^{2}$ : variância: 4 : desvio padrão; $c . v .:$ coeficiente de variação; $n$ : número de observações;

$2^{2} / d_{\text {artarar }}^{2}$ razăo entre variâncias das populações de alta e baixa produtividade

Os valores de $\mathrm{F}$ nos níveis de 5 e $1 \%$ de significância são 1,47 e 1,73, respectivamente (Steel \& Torrie, 1960). 
Apêndice 3. Parâmetros estatísticos da população de referência para citros com produtividade média de 43,09 t/ha e população de baixa produtividade ( 38,29 t/ha) em Matão/SP.

\begin{tabular}{|c|c|c|c|c|c|c|c|c|c|c|c|}
\hline \multirow{3}{*}{ Relação } & \multicolumn{10}{|c|}{ População } & \multirow{3}{*}{$e^{2}{ }_{a t e d} / d^{2}$ batsa } \\
\hline & \multicolumn{5}{|c|}{ Alta produtividade } & \multicolumn{5}{|c|}{ Baixa produtividade } & \\
\hline & $x$ & $4^{2}$ & $d$ & c.v. & $n$. & $x$ & $4^{2}$ & 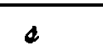 & c.v. & $n$ & \\
\hline$\overline{\mathrm{N} / \mathrm{P}}$ & 21,6966 & 2,5106 & 1,5844 & 0,0730 & 36 & 20,5113 & 3,2184 & 1,7939 & 0,0874 & 28 & 0,7800 \\
\hline$N / K$ & 1,7480 & 0,0341 & 0,1846 & 0,1056 & 36 & 1,6670 & 0,1021 & 0,3195 & 0,1916 & 28 & 0,3339 \\
\hline $\mathrm{N} / \mathrm{Ca}$ & 0,7283 & 0,0035 & 0,0594 & 0,0816 & 36 & 0,7104 & 0,0042 & 0,0649 & 0,0914 & 28 & 0,8364 \\
\hline $\mathrm{N} / \mathrm{Mg}$ & 6,8667 & 0,4283 & 0,6545 & 0,0953 & 36 & 6,5053 & 0,3211 & 0,5666 & 0,0871 & 28 & 1,3339 \\
\hline $\mathrm{N} / \mathrm{S}$ & 10,6522 & 0,4670 & 0,6834 & 0,0641 & 36 & 10,3394 & 0,7552 & 0,8690 & 0,0840 & 28 & 0,6183 \\
\hline $\mathrm{P} / \mathrm{N}$ & 0,0463 & 0,0000 & 0,0034 & 0,0738 & 36 & 0,0491 & 0,0000 & 0,0041 & 0,0836 & 28 & 0,5000 \\
\hline $\mathrm{P} / \mathrm{K}$ & 0,0808 & 0,0000 & 0,0091 & 0,1133 & 36 & 0,0811 & 0,0001 & 0,0127 & 0,1571 & 28 & 0,5000 \\
\hline $\mathrm{P} / \mathrm{Ca}$ & 0,0336 & 0,0000 & 0,0023 & 0,0711 & 36 & 0,0346 & 0,0000 & 0,0021 & 0,0629 & 28 & 0,0000 \\
\hline $\mathrm{P} / \mathrm{Mg}$ & 0,3167 & 0,0005 & 0,0238 & 0,0752 & 36 & 0,3180 & 0,0006 & 0,0255 & 0,0802 & 28 & 0,8769 \\
\hline $\mathrm{P} / \mathrm{S}$ & 0,4928 & 0,0016 & 0,0401 & 0,0814 & 36 & 0,5049 & 0,0008 & 0,0296 & 0,0586 & 28 & 1,8295 \\
\hline $\mathrm{K} / \mathrm{N}$ & 0,5782 & 0,0036 & 0,0605 & 0,1047 & 36 & 0,6181 & 0,0103 & 0,1015 & 0,1643 & 28 & 0,3556 \\
\hline $\mathrm{K} / \mathrm{P}$ & 12,5273 & 2,1475 & 1,4654 & 0,1169 & 36 & 12,5796 & 3,0242 & 1,7390 & 0,1382 & 28 & 0,7101 \\
\hline $\mathrm{K} / \mathrm{Ca}$ & 0,4218 & 0,0039 & 0,0625 & 0,1482 & 36 & 0,4375 & 0,0056 & 0,0751 & 0,1718 & 28 & 0,6920 \\
\hline $\mathrm{K} / \mathrm{Mg}$ & 3,9829 & 0,4304 & 0,6560 & 0,1647 & 36 & 4,0248 & 0,6109 & 0,7816 & 0,1942 & 28 & 0,7045 \\
\hline $\mathrm{K} / \mathrm{S}$ & 6,1554 & 0,5520 & 0,7430 & 0,1207 & 36 & 6,3539 & 0,9709 & 0,9853 & 0,1550 & 28 & 0,5685 \\
\hline $\mathrm{Ca} / \mathrm{N}$ & 1,3827 & 0,0150 & 0,1227 & 0,0888 & 36 & 1,4191 & 0,0170 & 0,1307 & 0,0921 & 28 & 0,8818 \\
\hline $\mathrm{Ca} / \mathrm{P}$ & 29,8884 & 4,6458 & 2,1554 & 0,0721 & 36 & 28,9400 & 3,1986 & 1,7884 & 0,0618 & 28 & 1,4524 \\
\hline $\mathrm{Ca} / \mathrm{K}$ & 2,4202 & 0,1221 & 0,3495 & 0,1444 & 36 & 2,3549 & 0,1836 & 0,4285 & 0,1819 & 28 & 0,6651 \\
\hline $\mathrm{Ca} / \mathrm{Mg}$ & 9,4224 & 0,1008 & 0,3176 & 0,0337 & 36 & 9,1652 & 0,0864 & 0,2940 & 0,0320 & 28 & 1,1667 \\
\hline $\mathrm{Ca} / \mathrm{S}$ & 14,6694 & 0,7124 & 0,8440 & 0,0575 & 36 & 14,5700 & 0,1967 & 0,4435 & 0,0304 & 28 & $3,6214^{* *}$ \\
\hline $\mathrm{Mg} / \mathrm{N}$ & 0,1469 & 0,0002 & 0,0148 & 0,1007 & 36 & 0,1548 & 0,0001 & 0,0135 & 0,0876 & 28 & 1,2222 \\
\hline $\mathrm{Mg} / \mathrm{P}$ & 3,1744 & 0,0555 & 0,2357 & 0,0742 & 36 & 3,1622 & 0,0563 & 0,2372 & 0,0750 & 28 & 0,9872 \\
\hline $\mathrm{Mg} / \mathrm{K}$ & 0,2576 & 0,0017 & 0,0414 & 0,1610 & 36 & 0,2581 & 0,0028 & 0,0535 & 0,2074 & 28 & 0,5993 \\
\hline $\mathrm{Mg} / \mathrm{Ca}$ & 0,1062 & 0,0000 & 0,0035 & 0,0335 & 36 & 0,1092 & 0,0000 & 0,0035 & 0,0320 & 28 & 1,0000 \\
\hline $\mathrm{Mg} / \mathrm{S}$ & 1,5606 & 0,0170 & 0,1306 & 0,0837 & 36 & 1,5919 & 0,0072 & 0,0852 & 0,0535 & 28 & $2,3526 *$ \\
\hline $\mathrm{S} / \mathrm{N}$ & 0,0942 & 0,0000 & 0,0062 & 0,0662 & 36 & 0,0973 & 0,0000 & 0,0081 & 0,0840 & 28 & 0,5714 \\
\hline $\mathrm{S} / \mathrm{P}$ & 2,0425 & 0,0296 & 0,1721 & 0,0843 & 36 & 1,9866 & 0,0128 & 0,1133 & 0,0570 & 28 & $2,3091^{*}$ \\
\hline $\mathbf{S} / \mathbf{K}$ & 0,1646 & 0,0003 & 0,0183 & 0,1117 & 36 & 0,1611 & 0,0006 & 0,0258 & 0,1604 & 28 & 0,5074 \\
\hline $\mathrm{S} / \mathrm{Ca}$ & 0,0683 & 0,0000 & 0,0039 & 0,0577 & 36 & 0,0686 & 0,0000 & 0,0020 & 0,0300 & 28 & 0,0000 \\
\hline $\mathrm{S} / \mathrm{Mg}$ & 0,6452 & 0,0030 & 0,0554 & 0,0859 & 36 & 0,6298 & 0,0011 & 0,0333 & 0,0529 & 28 & $2,7747^{*}$ \\
\hline $\mathbf{N}$ & 27,7800 & 1,9300 & 1,3910 & 0,0500 & 36 & 26,6000 & 2,9700 & 1,7240 & 0,0648 & 28 & 0,6506 \\
\hline $\mathbf{P}$ & 1,2830 & 0,0000 & 0,0560 & 0,0436 & 36 & 1,3000 & 0,0000 & 0,0540 & 0,0418 & 28 & 1,0000 \\
\hline $\mathrm{K}$ & 16,0500 & 3,0200 & 1,7390 & 0,1083 & 36 & 16,3390 & 5,0400 & 2,2450 & 0,1374 & 28 & 0,6002 \\
\hline $\mathrm{Ca}$ & 38,2660 & 3,2500 & 1,8030 & 0,0471 & 36 & 37,5640 & 3,4100 & 1,8480 & 0,0492 & 28 & 0,9514 \\
\hline $\mathrm{Mg}$ & 4,0660 & 0,0600 & 0,2500 & 0,0616 & 36 & 4,1030 & 0,0600 . & 0,2530 & 0,0616 & 28 & 0,9843 \\
\hline S & 2,6130 & 0,0200 & 0,1450 & 0,0557 & 36 & 2,5780 & 0,0100 & 0,1100 & 0,0426 & 28 & 1,7500 \\
\hline
\end{tabular}

$x$ : média; $\iota^{2}$ : variância; $4:$ desvio padrão; c.v.: coeficiente de variação; $n .:$ número de observações;

$d^{2}{ }_{a t a s}^{2} / d^{2}$ razão entre variâncias das populações de alta e baixa produtividade

Os valores de F nos níveis de 5 e 1\% de significância são 1,47 e 1,73, respectivamente (Steel \& Torrie, 1960). 
Apêndice 4. Parâmetros estatísticos da população de referência para citros com produtividade média de 29,49 t/ha e população de baixa produtividade ( $24,69 \mathrm{t} / \mathrm{ha}$ ) em Monte Azul/SP.

\begin{tabular}{|c|c|c|c|c|c|c|c|c|c|c|c|}
\hline \multirow{3}{*}{ Relação } & \multicolumn{10}{|c|}{ População } & \multirow{3}{*}{ 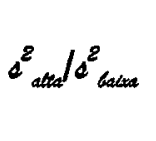 } \\
\hline & \multicolumn{5}{|c|}{ Alta produtividade } & \multicolumn{5}{|c|}{ Baixa produtividade } & \\
\hline & $x$ & $d^{2}$ & 2 & c.v. & $n$ & $x$ & $e^{2}$ & 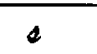 & c.v. & $n$. & \\
\hline $\mathrm{N} / \mathrm{P}$ & 19,1423 & 2,5027 & 1,5819 & 0,0826 & 38 & 15,8293 & 2,2703 & 1,5067 & 0,0951 & 26 & 1,1023 \\
\hline$N / K$ & 1,9932 & 0,0455 & 0,2133 & 0,1070 & 38 & 1,8257 & 0,0367 & 0,1917 & 0,1050 & 26 & 1,2376 \\
\hline $\mathrm{N} / \mathrm{Ca}$ & 0,5771 & 0,0005 & 0,0242 & 0,0420 & 38 & 0,5631 & 0,0006 & 0,0247 & 0,0439 & 26 & 0,9672 \\
\hline $\mathrm{N} / \mathrm{Mg}$ & 8,6680 & 0,4402 & 0,6635 & 0,0765 & 38 & 8,7667 & 0,7716 & 0,8784 & 0,1002 & 26 & 0,5705 \\
\hline $\mathrm{N} / \mathrm{S}$ & 10,2131 & 0,1930 & 0,4393 & 0,0430 & 38 & 9,2248 & 0,7626 & 0,8733 & 0,0946 & 26 & 0,2530 \\
\hline $\mathrm{P} / \mathrm{N}$ & 0,0526 & 0,0000 & 0,0045 & 0,0854 & 38 & 0,0637 & 0,0000 & 0,0062 & 0,0975 & 26 & 0,5000 \\
\hline $\mathrm{P} / \mathrm{K}$ & 0,1043 & 0,0000 & 0,0092 & 0,0890 & 38 & 0,1154 & 0,0000 & 0,0074 & 0,0641 & 26 & 1,8000 \\
\hline $\mathrm{P} / \mathrm{Ca}$ & 0,0303 & 0,0000 & 0,0029 & 0,0979 & 38 & 0,0358 & 0,0000 & 0,0031 & 0,0868 & 26 & 1,0000 \\
\hline $\mathrm{P} / \mathrm{Mg}$ & 0,4559 & 0,0026 & 0,0514 & 0,1127 & 38 & 0,5550 & 0,0018 & 0,0426 & 0,0768 & 26 & 1,4505 \\
\hline $\mathrm{P} / \mathrm{S}$ & 0,5370 & 0,0023 & 0,0486 & 0,0904 & 38 & 0,5833 & 0,0005 & 0,0239 & 0,0409 & 26 & $4,1403 * *$ \\
\hline $\mathrm{K} / \mathrm{N}$ & 0,5074 & 0,0030 & 0,0552 & 0,1088 & 38 & 0,5541 & 0,0040 & 0,0638 & 0,1152 & 26 & 0,7475 \\
\hline $\mathrm{K} / \mathrm{P}$ & 9,6643 & 0,7959 & 0,8921 & 0,0923 & 38 & 8,6983 & 0,3304 & 0,5748 & 0,0660 & 26 & $2,4083 *$ \\
\hline $\mathrm{K} / \mathrm{Ca}$ & 0,2934 & 0,0015 & 0,0388 & 0,1323 & 38 & 0,3113 & 0,0010 & 0,0316 & 0,1015 & 26 & 1,5100 \\
\hline $\mathrm{K} / \mathrm{Mg}$ & 4,4019 & 0,3581 & 0,5984 & 0,1359 & 38 & 4,8157 & 0,1130 & 0,3361 & 0,0698 & 26 & $3,1690 * *$ \\
\hline $\mathrm{K} / \mathrm{S}$ & 5,1826 & 0,3560 & 0,5966 & 0,1151 & 38 & 5,0705 & 0,1195 & 0,3457 & 0,0681 & 26 & $2,9780 * *$ \\
\hline $\mathrm{Ca} / \mathrm{N}$ & 1,7357 & 0,0053 & 0,0728 & 0,0419 & 38 & 1,7789 & 0,0061 & 0,0786 & 0,0441 & 26 & 0,8592 \\
\hline $\mathrm{Ca} / \mathrm{P}$ & 33,2472 & 11,3264 & 3,3654 & 0,1012 & 38 & 28,1042 & 5,3664 & 2,3165 & 0,0824 & 26 & $2,1105 *$ \\
\hline $\mathrm{Ca} / \mathrm{K}$ & 3,4683 & 0,2275 & 0,4770 & 0,1375 & 38 & 3,2413 & 0,0946 & 0,3076 & 0,0949 & 26 & $2,4039 *$ \\
\hline $\mathrm{Ca} / \mathrm{Mg}$ & 15,0175 & 0,8318 & 0,9120 & 0,0607 & 38 & 15,5594 & 1,7016 & 1,3044 & 0,0838 & 26 & 0,4888 \\
\hline $\mathrm{Ca} / \mathrm{S}$ & 17,7077 & 0,4048 & 0,6362 & 0,0359 & 38 & 16,3658 & 1,3608 & 1,1665 & 0,0712 & 26 & 0,2974 \\
\hline $\mathrm{Mg} / \mathrm{N}$ & 0,1160 & 0,0000 & 0,0089 & 0,0774 & 38 & 0,1152 & 0,0001 & 0,0120 & 0,1046 & 26 & 0,5333 \\
\hline $\mathrm{Mg} / \mathrm{P}$ & 2,2219 & 0,0704 & 0,2653 & 0,1194 & 38 & 1,8116 & 0,0188 & 0,1372 & 0,0757 & 26 & $3,7387 * *$ \\
\hline $\mathrm{Mg} / \mathrm{K}$ & 0,2315 & 0,0011 & 0,0338 & 0,1463 & 38 & 0,2085 & 0,0001 & 0,0136 & 0,0655 & 26 & $6,0526 * *$ \\
\hline $\mathrm{Mg} / \mathrm{Ca}$ & 0,0668 & 0,0000 & 0,0042 & 0,0639 & 38 & 0,0647 & 0,0000 & 0,0059 & 0,0911 & 26 & 0,6666 \\
\hline $\mathrm{Mg} / \mathrm{S}$ & 1,1818 & 0,0031 & 0,0559 & 0,0473 & 38 & 1,0552 & 0,0048 & 0,0699 & 0,0663 & 26 & 0,6400 \\
\hline $\mathbf{S} / \mathbf{N}$ & 0,0980 & 0,0000 & 0,0041 & 0,0427 & 38 & 0,1093 & 0,0001 & 0,0102 & 0,0936 & 26 & 0,2000 \\
\hline $\mathrm{S} / \mathrm{P}$ & 1,8777 & 0,0325 & 0,1804 & 0,0961 & 38 & 1,7171 & 0,0052 & 0,0721 & 0,0420 & 26 & $6,2634 * *$ \\
\hline $\mathbf{S} / \mathbf{K}$ & 0,1955 & 0,0005 & 0,0236 & 0,1210 & 38 & 0,1980 & 0,0001 & 0,0131 & 0,0664 & 26 & $3,2941 * *$ \\
\hline $\mathrm{S} / \mathrm{Ca}$ & 0,0565 & 0,0000 & 0,0021 & 0,0370 & 38 & 0,0614 & 0,0000 & 0,0044 & 0,0730 & 26 & 0,0000 \\
\hline $\mathrm{S} / \mathrm{Mg}$ & 0,8479 & 0,0015 & 0,0396 & 0,0467 & 38 & 0,9517 & 0,0040 & 0,0638 & 0,0670 & 26 & 0,3848 \\
\hline$N$ & 24,4280 & 0,0027 & 0,5220 & 0,0213 & 38 & 23,5260 & 0,0069 & 0,8310 & 0,0353 & 26 & 0,3945 \\
\hline $\mathbf{P}$ & 1,2840 & 0,0001 & 0,1020 & 0,0800 & 38 & 1,4960 & 0,0001 & 0,1140 & 0,0767 & 26 & 0,8461 \\
\hline $\mathbf{K}$ & 12,4000 & 0,0196 & 1,4000 & 0,1129 & 38 & 13,0030 & 0,0149 & 1,2210 & 0,0939 & 26 & 1,3150 \\
\hline $\mathrm{Ca}$ & 42,3730 & 0,0145 & 1,2070 & 0,0285 & 38 & 41,7960 & 0,0057 & 0,7590 & 0,0181 & 26 & $2,5286 *$ \\
\hline Mg & 2,8310 & 0,0003 & 0,1860 & 0,0657 & 38 & 2,7030 & 0,0004 & 0,2210 & 0,0820 & 26 & 0,7142 \\
\hline $\mathbf{S}$ & 2,3940 & 0,0000 & 0,0760 & 0,0321 & 38 & 2,5650 & 0,0003 & 0,1710 & 0,0670 & 26 & 0,2000 \\
\hline
\end{tabular}

$x$ : média; $\iota^{2}$ : variância; $\&$ : desvio padrão; $c$.v.: coeficiente de variação; $n .:$ número de observações;

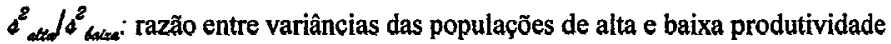

Os valores de $\mathrm{F}$ nos níveis de 5 e $1 \%$ de significância são 1,47 e 1,73, respectivamente (Steel \& Torrie, 1960). 
Apêndice 5. Parâmetros estatísticos da população de referência para citros com produtividade média de 27,51 tha e população de baixa produtividade ( $25,52 \mathrm{t} / \mathrm{ha}$ ) em Olímpia/SP.

\begin{tabular}{|c|c|c|c|c|c|c|c|c|c|c|c|}
\hline \multirow{3}{*}{ Relação } & \multicolumn{10}{|c|}{ População } & \multirow{3}{*}{$4_{\text {altad }}^{z} / d_{\text {batia }}^{2}$} \\
\hline & \multicolumn{5}{|c|}{ Alta produtividade } & \multicolumn{5}{|c|}{ Baixa produtividade } & \\
\hline & $x$ & $4^{2}$ & $c$ & c.v. & $n$ & $x$ & $e^{2}$ & $a$ & c.v. & $n$. & \\
\hline$\overline{\mathrm{N} / \mathrm{P}}$ & 22,3096 & 1,8252 & 1,3510 & 0,0605 & 29 & 21,0074 & 0,8740 & 0,9349 & 0,0445 & 35 & $2,0882^{*}$ \\
\hline $\mathbf{N} / \mathbf{K}$ & 2,5521 & 0,0764 & 0,2764 & 0,1083 & 29 & 2,4212 & 0,0388 & 0,1970 & 0,0813 & 35 & $1,9688 *$ \\
\hline $\mathrm{N} / \mathrm{Ca}$ & 0,9425 & 0,0032 & 0,0566 & 0,0600 & 29 & 0,8771 & 0,0008 & 0,0286 & 0,0326 & 35 & $3,9146 * *$ \\
\hline $\mathrm{N} / \mathrm{Mg}$ & 6,7924 & 0,2012 & 0,4486 & 0,0660 & 29 & 6,2769 & 0,0748 & 0,2734 & 0,0435 & 35 & $2,6907^{* *}$ \\
\hline$N / S$ & 10,1704 & 0,6738 & 0,8209 & 0,0807 & 29 & 9,4525 & 0,1682 & 0,4102 & 0,0434 & 35 & $4,0044 * *$ \\
\hline $\mathrm{P} / \mathrm{N}$ & 0,0449 & 0,0000 & 0,0026 & 0,0582 & 29 & 0,0476 & 0,0000 & 0,0020 & 0,0437 & 35 & 0,0000 \\
\hline $\mathrm{P} / \mathrm{K}$ & 0,1142 & 0,0000 & 0,0075 & 0,0662 & 29 & 0,1152 & 0,0000 & 0,0065 & 0,0572 & 35 & 1,5000 \\
\hline $\mathrm{P} / \mathrm{Ca}$ & 0,0423 & 0,0000 & 0,0024 & 0,0578 & 29 & $.0,0418$ & 0,0000 & 0,0021 & 0,0518 & 35 & 0,0000 \\
\hline $\mathrm{P} / \mathrm{Mg}$ & 0,3049 & 0,0003 & 0,0196 & 0,0643 & 29 & 0,2992 & 0,0002 & 0,0158 & 0,0530 & 35 & 1,5200 \\
\hline $\mathrm{P} / \mathrm{S}$ & 0,4560 & 0,0006 & 0,0260 & 0,0570 & 29 & 0,4502 & 0,0002 & 0,0167 & 0,0370 & 35 & $2,4285 * *$ \\
\hline $\mathrm{K} / \mathrm{N}$ & 0,3961 & 0,0017 & 0,0413 & 0,1044 & 29 & 0,4154 & 0,0009 & 0,0305 & 0,0735 & 35 & $1,8387 *$ \\
\hline $\mathrm{K} / \mathrm{P}$ & 8,7934 & 0,3426 & 0,5853 & 0,0665 & 29 & 8,7065 & 0,2145 & 0,4632 & 0,0532 & 35 & 1,5972 \\
\hline $\mathrm{K} / \mathrm{Ca}$ & 0,3729 & 0,0016 & 0,0411 & 0,1102 & 29 & 0,3646 & 0,0010 & 0,0323 & 0,0885 & 35 & 1,6250 \\
\hline $\mathrm{K} / \mathrm{Mg}$ & 2,6861 & 0,0827 & 0,2877 & 0,1071 & 29 & 2,6087 & 0,0534 & 0,2312 & 0,0886 & 35 & 1,5475 \\
\hline $\mathrm{K} / \mathrm{S}$ & 4,0142 & 0,1578 & 0,3973 & 0,0989 & 29 & 3,9215 & 0,0721 & 0,2686 & 0,0685 & 35 & $2,1870 *$ \\
\hline $\mathrm{Ca} / \mathrm{N}$ & 1,0644 & 0,0037 & 0,0607 & 0,0571 & 29 & 1,1411 & 0,0012 & 0,0357 & 0,0313 & 35 & $2,8906 * *$ \\
\hline $\mathrm{Ca} / \mathrm{P}$ & 23,7067 & 1,9072 & 1,3810 & 0,0582 & 29 & 23,9694 & 1,5500 & 1,2450 & 0,0519 & 35 & 1,2304 \\
\hline $\mathrm{Ca} / \mathrm{K}$ & 2,7137 & 0,0957 & 0,3093 & 0,1140 & 29 & 2,7652 & 0,0724 & 0,2691 & 0,0973 & 35 & 1,3210 \\
\hline $\mathrm{Ca} / \mathrm{Mg}$ & 7,2062 & 0,0360 & 0,1897 & 0,0263 & 29 & 7,1555 & 0,0381 & 0,1953 & 0,0273 & 35 & 0,9439 \\
\hline $\mathrm{Ca} / \mathrm{S}$ & 10,7861 & 0,2199 & 0,4689 & 0,0434 & 29 & 10,7794 & 0,1738 & 0,4169 & 0,0386 & 35 & 1,2649 \\
\hline $\mathrm{Mg} / \mathrm{N}$ & 0,1478 & 0,0000 & 0,0093 & 0,0635 & 29 & 0,1596 & 0,0000 & 0,0068 & 0,0427 & 35 & 1,8000 \\
\hline $\mathrm{Mg} / \mathrm{P}$ & 3,2920 & 0,0449 & 0,2120 & 0,0644 & 29 & 3,3512 & 0,0325 & 0,1803 & 0,0538 & 35 & 1,3822 \\
\hline $\mathrm{Mg} / \mathrm{K}$ & 0,3766 & 0,0018 & 0,0428 & 0,1138 & 29 & 0,3867 & 0,0015 & 0,0394 & 0,1019 & 35 & 1,1871 \\
\hline $\mathrm{Mg} / \mathrm{Ca}$ & 0,1388 & 0,0000 & 0,0037 & 0,0272 & 29 & 0,1398 & 0,0000 & 0,0038 & 0,0276 & 35 & 1,0000 \\
\hline $\mathrm{Mg} / \mathrm{S}$ & 1,4970 & 0,0033 & 0,0576 & 0,0384 & 29 & 1,5066 & 0,0024 & 0,0497 & 0,0329 & 35 & 1,3441 \\
\hline $\mathbf{S} / \mathbf{N}$ & 0,0988 & 0,0000 & 0,0073 & 0,0744 & 29 & 0,1059 & 0,0000 & 0,0046 & 0,0439 & 35 & $2,5000 * *$ \\
\hline $\mathrm{S} / \mathrm{P}$ & 2,1996 & 0,0148 & 0,1218 & 0,0554 & 29 & 2,2238 & 0,0066 & 0,0812 & 0,0365 & 35 & $2,2500 *$ \\
\hline $\mathrm{S} / \mathrm{K}$ & 0,2514 & 0,0006 & 0,0249 & 0,0993 & 29 & 0,2562 & 0,0003 & 0,0191 & 0,0746 & 35 & 1,6756 \\
\hline $\mathrm{S} / \mathrm{Ca}$ & 0,0928 & 0,0000 & 0,0040 & 0,0436 & 29 & 0,0929 & 0,0000 & 0,0036 & 0,0396 & 35 & $2,0000 *$ \\
\hline $\mathrm{S} / \mathrm{Mg}$ & 0,6689 & 0,0006 & 0,0257 & 0,0385 & 29 & 0,6644 & 0,0004 & 0,0213 & 0,0320 & 35 & 1,4666 \\
\hline $\mathrm{N}$ & 29,1000 & 0,0133 & 1,1560 & 0,0397 & 29 & 28,5910 & 0,0067 & 0,8230 & 0,0287 & 35 & $1,9734^{*}$ \\
\hline $\mathbf{P}$ & 1,3060 & 0,0000 & 0,0590 & 0,0454 & 29 & 1,3620 & 0,0000 & 0,0540 & 0,0401 & 35 & 1,3333 \\
\hline K & 11,5030 & 0,0109 & 1,0480 & 0,0911 & 29 & 11,8770 & 0,0084 & 0,9190 & 0,0774 & 35 & 1,2994 \\
\hline $\mathrm{Ca}$ & 30,9440 & 0,0278 & 1,6690 & 0,0539 & 29 & 32,6170 & 0,0127 & 1,1290 & 0,0346 & 35 & $2,1858 *$ \\
\hline $\mathbf{M g}$ & 4,2960 & 0,0006 & 0,2480 & 0,0578 & 29 & 4,5600 & 0,0002 & 0,1570 & 0,0345 & 35 & $2,4800 * *$ \\
\hline $\mathbf{S}$ & 2,8720 & 0,0002 & 0,1680 & 0,0587 & 29 & 3,0280 & 0,0001 & 0,1170 & 0,0388 & 35 & 2,0000 \\
\hline
\end{tabular}

$x$ : média; $\AA^{2}$ : variância: «: desvio padrão; c.v.: coeficiente de variação; $n$. número de observações;

$4^{2}{ }^{2} e^{2}$ razão entre variâncias das populaçōes de alta e baixa produtividade

Os valores de F nos níveis de 5 e 1\% de significância são 1,47 e 1,73, respectivamente (Steel \& Torrie, 1960). 
Apêndice 6. Parâmetros estatísticos da população de referência para citros com produtividade média de $38,87 \mathrm{t} / \mathrm{ha}$ e população de baixa produtividade ( $34,91 \mathrm{t} / \mathrm{ha}$ ) em Pirassununga/SP.

\begin{tabular}{|c|c|c|c|c|c|c|c|c|c|c|c|}
\hline \multirow{3}{*}{ Relação } & \multicolumn{10}{|c|}{ População } & \multirow{3}{*}{$e^{2}$ alted $/ e^{2}$} \\
\hline & \multicolumn{5}{|c|}{ Alta produtividade } & \multicolumn{5}{|c|}{ Baixa produtividade } & \\
\hline & $x$ & $e^{2}$ & 4 & c.v. & $n$ & $x$ & $e^{2}$ & 4 & c.v. & $n$ & \\
\hline $\mathrm{N} / \mathrm{P}$ & 20,6748 & 0,9711 & 0,9854 & 0,0476 & 36 & 19,0864 & 1,4878 & 1,2197 & 0,0639 & 28 & 0,6527 \\
\hline $\mathbf{N} / \mathbf{K}$ & 2,4553 & 0,0840 & 0,2899 & 0,1181 & 36 & 2,2431 & 0,2417 & 0,4916 & 0,2191 & 28 & 0,3479 \\
\hline $\mathrm{N} / \mathrm{Ca}$ & 0,5414 & 0,0014 & 0,0384 & 0,0710 & 36 & 0,5439 & 0,0013 & 0,0372 & 0,0684 & 28 & 1,0725 \\
\hline $\mathrm{N} / \mathrm{Mg}$ & 5,6688 & 0,0707 & 0,2659 & 0,0469 & 36 & 6,0477 & 0,1106 & 0,3326 & 0,0550 & 28 & 0,6394 \\
\hline$N / S$ & 10,5220 & 0,1064 & 0,3263 & 0,0310 & 36 & 10,4717 & 0,4597 & 0,6780 & 0,0647 & 28 & 0,2316 \\
\hline $\mathbf{P} / \mathbf{N}$ & 0,0484 & 0,0000 & 0,0023 & 0,0486 & 36 & 0,0526 & 0,0001 & 0,0034 & 0,0650 & 28 & 1,0000 \\
\hline $\mathrm{P} / \mathrm{K}$ & 0,1189 & 0,0002 & 0,0153 & 0,1285 & 36 & 0,1169 & 0,0004 & 0,0220 & 0,1882 & 28 & 0,4792 \\
\hline $\mathrm{P} / \mathrm{Ca}$ & 0,0262 & 0,0000 & 0,0020 & 0,0771 & 36 & 0,0286 & 0,0001 & 0,0025 & 0,0892 & 28 & 0,0000 \\
\hline $\mathrm{P} / \mathrm{Mg}$ & 0,2746 & 0,0002 & 0,0168 & 0,0611 & 36 & 0,3187 & 0,0011 & 0,0335 & 0,1051 & 28 & 0,2500 \\
\hline $\mathrm{P} / \mathrm{S}$ & 0,5099 & 0,0007 & 0,0265 & 0,0521 & 36 & 0,5499 & 0,0014 & 0,0376 & 0,0685 & 28 & 0,5000 \\
\hline $\mathrm{K} / \mathrm{N}$ & 0,4129 & 0,0024 & 0,0496 & 0,1201 & 36 & 0,4671 & 0,0105 & 0,1026 & 0,2196 & 28 & 0,2336 \\
\hline $\mathrm{K} / \mathbf{P}$ & 8,5294 & 1,0146 & 1,0072 & 0,1180 & 36 & 8,8328 & 2,4971 & 1,5802 & 0,1789 & 28 & 0,4063 \\
\hline $\mathrm{K} / \mathrm{Ca}$ & 0,2241 & 0,0011 & 0,0343 & 0,1532 & 36 & 0,2534 & 0,0029 & 0,0542 & 0,2139 & 28 & 0,4014 \\
\hline $\mathrm{K} / \mathrm{Mg}$ & 2,3489 & 0,1288 & 0,3589 & 0,1528 & 36 & 2,8451 & 0,5339 & 0,7307 & 0,2568 & 28 & 0,2412 \\
\hline $\mathrm{K} / \mathrm{S}$ & 4,3473 & 0,3047 & 0,5520 & 0,1269 & 36 & 4,8417 & 0,7317 & 0,8554 & 0,1766 & 28 & 0,4165 \\
\hline $\mathrm{Ca} / \mathrm{N}$ & 1,8558 & 0,0171 & 0,1310 & 0,0706 & 36 & 1,8463 & 0,0147 & 0,1212 & 0,0656 & 28 & 1,1680 \\
\hline $\mathrm{Ca} / \mathrm{P}$ & 38,3417 & 8,4245 & 2,9025 & 0,0757 & 36 & 35,2313 & 10,1553 & 3,1867 & 0,0904 & 28 & 0,8296 \\
\hline $\mathrm{Ca} / \mathrm{K}$ & 4,5732 & 0,5885 & 0,7671 & 0,1677 & 36 & 4,1344 & 0,8716 & 0,9336 & 0,2258 & 28 & 0,6752 \\
\hline $\mathrm{Ca} / \mathrm{Mg}$ & 10,5014 & 0,3750 & 0,6123 & 0,0583 & 36 & 11,1473 & 0,5123 & 0,7157 & 0,0642 & 28 & 0,7320 \\
\hline $\mathrm{Ca} / \mathrm{S}$ & 19,4923 & 0,8502 & 0,9221 & 0,0473 & 36 & 19,2825 & 1,1601 & 1,0771 & 0,0558 & 28 & 0,7329 \\
\hline $\mathrm{Mg} / \mathrm{N}$ & 0,1767 & 0,0000 & 0,0085 & 0,0486 & 36 & 0,1658 & 0,0001 & 0,0093 & 0,0563 & 28 & 0,7778 \\
\hline $\mathrm{Mg} / \mathrm{P}$ & 3,6533 & 0,0462 & 0,2149 & 0,0588 & 36 & 3,1711 & 0,1104 & 0,3324 & 0,1048 & 28 & 0,4182 \\
\hline $\mathrm{Mg} / \mathrm{K}$ & 0,4357 & 0,0046 & 0,0685 & 0,1572 & 36 & 0,3746 & 0,0091 & 0,0958 & 0,2559 & 28 & 0,5103 \\
\hline $\mathrm{Mg} / \mathrm{Ca}$ & 0,0955 & 0,0000 & 0,0058 & 0,0606 & 36 & 0,0900 & 0,0001 & 0,0056 & 0,0622 & 28 & 1,0000 \\
\hline $\mathrm{Mg} / \mathrm{S}$ & 1,8586 & 0,0052 & 0,0722 & 0,0388 & 36 & 1,7367 & 0,0218 & 0,1476 & 0,0850 & 28 & 0,2393 \\
\hline $\mathbf{S} / \mathbf{N}$ & 0,0951 & 0,0000 & 0,0029 & 0,0307 & 36 & 0,0958 & 0,0001 & 0,0061 & 0,0636 & 28 & 0,2500 \\
\hline $\mathrm{S} / \mathrm{P}$ & 1,9662 & 0,0105 & 0,1027 & 0,0522 & 36 & 1,8269 & 0,0162 & 0,1274 & 0,0697 & 28 & 0,6503 \\
\hline $\mathbf{S} / \mathbf{K}$ & 0,2337 & 0,0009 & 0,0306 & 0,1312 & 36 & 0,2128 & 0,0014 & 0,0376 & 0,1768 & 28 & 0,6620 \\
\hline S/Ca & 0,0514 & 0,0000 & 0,0024 & 0,0480 & 36 & 0,0520 & 0,0001 & 0,0028 & 0,0537 & 28 & 1,0000 \\
\hline $\mathrm{S} / \mathrm{Mg}$ & 0,5388 & 0,0004 & 0,0209 & 0,0389 & 36 & 0,5798 & 0,0024 & 0,0498 & 0,0859 & 28 & 0,1774 \\
\hline $\mathbf{N}$ & 24,0190 & 0,0042 & 0,6530 & 0,0272 & 36 & 23,7960 & 0,0133 & 1,1530 & 0,0484 & 28 & 0,3208 \\
\hline $\mathbf{P}$ & 1,1630 & 0,0000 & 0,0540 & 0,0466 & 36 & 1,2500 & 0,0001 & 0,0740 & 0,0596 & 28 & 0,5000 \\
\hline $\mathbf{K}$ & 9,9160 & 0,0141 & 1,1870 & 0,1197 & 36 & 11,0320 & 0,0421 & 2,0530 & 0,1861 & 28 & 0,3344 \\
\hline $\mathrm{Ca}$ & 44,5020 & 0,0458 & 2,1420 & 0,0481 & 36 & 43,8320 & 0,0364 & 1,9100 & 0,0435 & 28 & 1,2580 \\
\hline $\mathrm{Mg}$ & 4,2440 & 0,0003 & 0,1910 & 0,0452 & 36 & 3,9460 & 0,0008 & 0,2870 & 0,0728 & 28 & 0,4458 \\
\hline $\mathbf{S}$ & 2,2830 & 0,0000 & 0,0370 & 0,0165 & 36 & 2,2750 & 0,0001 & 0,0510 & 0,0227 & 28 & 0,3333 \\
\hline
\end{tabular}

$x$ : média; $\iota^{2}$ : variância 屯: desvio padrão; $c . v$.: coeficiente de variação; $n .:$ número de observações;

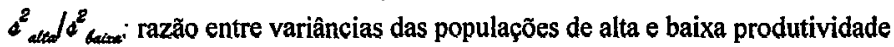

Os valores de F nos níveis de 5 e 1\% de significância são 1,47 e 1,73, respectivamente (Steel \& Torrie, 1960). 
Apêndice 7. Parâmetros estatísticos da população de referência para citros. População de produtividade média na faixa compreendidade entre a média menos um desvio padrão e a média mais um desvio padrão (produtividade média de $33,64 \mathrm{t} / \mathrm{ha}$ ) e população de baixa produtividade (produtividade média de 19,43 t/ha).

\begin{tabular}{|c|c|c|c|c|c|c|c|c|c|c|c|}
\hline \multirow{3}{*}{ Relação } & \multicolumn{10}{|c|}{ População } & \multirow{3}{*}{$c^{2}{ }_{a t a d} / d^{2}$ batian } \\
\hline & \multicolumn{5}{|c|}{ Alta produtividade } & \multicolumn{5}{|c|}{ Baixa produtividade } & \\
\hline & $x$ & $e^{2}$ & 4 & c.v. & $n$. & $x$ & $d^{2}$ & 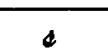 & c.v. & $n$ & \\
\hline $\mathrm{N} / \mathrm{P}$ & 21,0106 & 14,2305 & 3,7723 & 17,9545 & 156 & 21,4038 & 55,2976 & 7,4362 & 34,7426 & 75 & 0,2573 \\
\hline N/K & 2,1415 & 0,1900 & 0,4359 & 20,3541 & 156 & 2,2436 & 0,1434 & 0,3787 & 16,8811 & 75 & 1,3246 \\
\hline $\mathrm{N} / \mathrm{Ca}$ & 0,6939 & 0,0154 & 0,1240 & 17,8709 & 156 & 0,6668 & 0,0132 & 0,1148 & 17,2178 & 75 & 1,1669 \\
\hline $\mathrm{N} / \mathrm{Mg}$ & 8,3730 & 3,4908 & 1,8684 & 22,3140 & 156 & 8,0671 & 4,9458 & 2,2239 & 27,5678 & 75 & 0,7058 \\
\hline $\mathrm{N} / \mathrm{S}$ & 9,8176 & 1,7739 & 1,3319 & 13,5663 & 122 & 9,5787 & 1,3806 & 1,1750 & 12,2668 & 49 & 1,2849 \\
\hline $\mathbf{P} / \mathbf{N}$ & 0,0495 & 0,0001 & 0,0111 & 22,3605 & 156 & 0,0506 & 0,0002 & 0,0131 & 25,9081 & 75 & 0,7059 \\
\hline $\mathrm{P} / \mathrm{K}$ & 0,1067 & 0,0014 & 0,0369 & 34,5756 & 156 & 0,1124 & 0,0011 & 0,0332 & 29,5570 & 75 & 1,2364 \\
\hline $\mathrm{P} / \mathrm{Ca}$ & 0,0341 & 0,0001 & 0,0083 & 24,3820 & 156 & 0,0336 & 0,0001 & 0,0105 & 31,3330 & 75 & 0,6364 \\
\hline $\mathrm{P} / \mathrm{Mg}$ & 0,4190 & 0,0215 & 0,1468 & 35,0260 & 156 & 0,4168 & 0,0325 & 0,1803 & 43,2621 & 75 & 0,6626 \\
\hline $\mathrm{P} / \mathrm{S}$ & 0,4385 & 0,0034 & 0,0587 & 13,3844 & 122 & 0,4193 & 0,0079 & 0,0887 & 21,1429 & 49 & 0,4377 \\
\hline $\mathrm{K} / \mathrm{N}$ & 0,4843 & 0,0084 & 0,0916 & 18,9030 & 156 & 0,4585 & 0,0065 & 0,0803 & 17,5238 & 75 & 1,2992 \\
\hline $\mathrm{K} / \mathrm{P}$ & 10,1754 & 6,1413 & 2,4782 & 24,3546 & 156 & 9,6842 & 10,6055 & 3,2566 & 33,6281 & 75 & 0,5791 \\
\hline $\mathrm{K} / \mathrm{Ca}$ & 0,3388 & 0,0094 & 0,0971 & 28,6690 & 156 & 0,3035 & 0,0041 & 0,0638 & 21,0332 & 75 & $2,3194 * *$ \\
\hline $\mathrm{K} / \mathrm{Mg}$ & 4,0694 & 1,5026 & 1,2258 & 30,1223 & 156 & 3,7346 & 1,8938 & 1,3762 & 36,8484 & 75 & 0,7934 \\
\hline $\mathbf{K} / \mathbf{S}$ & 4,9025 & 1,0326 & 1,0162 & 20,7279 & 122 & 4,4268 & 0,6504 & 0,8065 & 18,2173 & 49 & $1,5878 *$ \\
\hline $\mathrm{Ca} / \mathrm{N}$ & 1,4882 & 0,0763 & 0,2762 & 18,5591 & 156 & 1,5464 & 0,0789 & 0,2810 & 18,1691 & 75 & 0,9664 \\
\hline $\mathrm{Ca} / \mathrm{P}$ & 31,0993 & 58,7910 & 7,6675 & 24,6550 & 156 & 32,7571 & 116,5514 & 10,7959 & 32,9575 & 75 & 0,5044 \\
\hline $\mathrm{Ca} / \mathrm{K}$ & 3,2157 & 0,9781 & 0,9890 & 30,7555 & 156 & 3,4439 & 0,5436 & 0,7373 & 21,4082 & 75 & $1,7994^{* *}$ \\
\hline $\mathrm{Ca} / \mathrm{Mg}$ & 12,3114 & 9,5071 & 3,0834 & 25,0447 & 156 & 12,2050 & 12,3827 & 3,5189 & 28,8316 & 75 & 0,7678 \\
\hline $\mathrm{Ca} / \mathrm{S}$ & 14,3702 & 13,4505 & 3,6675 & 25,5216 & 122 & 15,1123 & 14,4286 & 3,7985 & 25,1351 & 49 & 0,9322 \\
\hline $\mathrm{Mg} / \mathrm{N}$ & 0,1257 & 0,0009 & 0,0292 & 23,2002 & 156 & 0,1347 & 0,0018 & 0,0422 & 31,3658 & 75 & 0,4775 \\
\hline $\mathrm{Mg} / \mathrm{P}$ & 2,6673 & 0,7594 & 0,8714 & 32,6704 & 156 & 2,9288 & 1,9186 & 1,3851 & 47,2937 & 75 & 0,3958 \\
\hline $\mathrm{Mg} / \mathrm{K}$ & 0,2706 & 0,0083 & 0,0910 & 33,6195 & 156 & 0,3037 & 0,0119 & 0,1092 & 35,9475 & 75 & 0,6946 \\
\hline $\mathrm{Mg} / \mathrm{Ca}$ & 0,0860 & 0,0004 & 0,0201 & 23,3848 & 156 & 0,0872 & 0,0004 & 0,0199 & 22,8672 & 75 & 1,0000 \\
\hline $\mathrm{Mg} / \mathrm{S}$ & 1,2832 & 0,1618 & 0,4022 & 31,3443 & 122 & 1,4349 & 0,2718 & 0,5214 & 36,3369 & 49 & 0,5951 \\
\hline $\mathbf{S} / \mathbf{N}$ & 0,1037 & 0,0002 & 0,0135 & 13,0524 & 122 & 0,1059 & 0,0002 & 0,0123 & 11,5790 & 49 & 1,2000 \\
\hline $\mathbf{S} / \mathbf{P}$ & 2,3215 & 0,0974 & 0,3121 & 13,4434 & 122 & 2,5468 & 0,7897 & 0,8886 & 34,8918 & 49 & 0,1233 \\
\hline $\mathrm{S} / \mathrm{K}$ & 0,2134 & 0,0025 & 0,0496 & 23,2527 & 122 & 0,2330 & 0,0017 & 0,0407 & 17,4509 & 49 & $1,4909 *$ \\
\hline $\mathrm{S} / \mathrm{Ca}$ & 0,0738 & 0,0003 & 0,0173 & 23,4379 & 122 & 0,0700 & 0,0003 & 0,0159 & 22,6409 & 49 & 1,2000 \\
\hline $\mathrm{S} / \mathrm{Mg}$ & 0,8582 & 0,0725 & 0,2693 & 31,3842 & 122 & 0,7841 & 0,0703 & 0,2651 & 33,8150 & 49 & 1,0319 \\
\hline $\mathrm{N}$ & 25,9330 & 0,0455 & 2,1330 & 8,2260 & 156 & 25,6840 & 0,0429 & 2,0720 & 8,0661 & 75 & 1,0603 \\
\hline $\mathbf{P}$ & 1,2800 & 0,0009 & 0,2920 & 22,7865 & 156 & 1,2930 & 0,0012 & 0,3480 & 26,8846 & 75 & 0,7025 \\
\hline K & 12,4830 & 0,0431 & 2,0760 & 16,6312 & 156 & 11,6760 & 0,0248 & 1,5750 & 13,4903 & 75 & $1,7372 * *$ \\
\hline $\mathrm{Ca}$ & 38,4150 & 0,4595 & 6,7790 & 17,6465 & 156 & 39,5440 & 0,4878 & 6,9840 & 17,6611 & 75 & 0,9422 \\
\hline $\mathrm{Mg}$ & 3,2650 & 0,0070 & 0,8390 & 25,6826 & 156 & 3,4800 & 0,0138 & 1,1740 & 33,7468 & 75 & 0,5098 \\
\hline S & 2,6750 & 0,0013 & 0,3600 & 13,4470 & 122 & 2,7160 & 0,0010 & 0,3090 & 11,3804 & 49 & 1,3438 \\
\hline
\end{tabular}

$x$ : média; $\AA^{2}:$ variância đ: desvio padrão; $c$. $v$.: coeficiente de variação; $n .:$ número de observações;

$2^{2} d e^{2}$. razão entre variâncias das populações de alta e baixa produtividade

Os valores de $\mathrm{F}$ nos níveis de 5 e 1\% de significância são 1,47 e 1,73, respectivamente (Steel \& Torrie, 1960). 
Apêndice 8. Parâmetros estatísticos da população de referência para citros. População de produtividade média na faixa compreendidade entre a média menos o $2 / 3$ do desvio padrão e a média mais $2 / 3$ do desvio padrão (produtividade média de 33,84 t/ha) e população de baixa produtividade (produtividade média de 22,23 tha).

\begin{tabular}{|c|c|c|c|c|c|c|c|c|c|c|c|}
\hline \multirow{3}{*}{ Relação } & \multicolumn{10}{|c|}{ População } & \multirow{3}{*}{$d^{2}{ }_{\text {attea }} / d^{2}$ baiste } \\
\hline & \multicolumn{5}{|c|}{ Alta produtividade } & \multicolumn{5}{|c|}{ Baixa produtividade } & \\
\hline & $x$ & $e^{2}$ & $d$ & c.v. & $n$ & $x$ & $d^{2}$ & $c$ & c.v. & $n$. & \\
\hline $\mathrm{N} / \mathrm{P}$ & 21,2523 & 13,5431 & 3,6801 & 17,3162 & 93 & 21,1844 & 39,3296 & 6,2713 & 29,6036 & 126 & 0,3443 \\
\hline $\mathrm{N} / \mathrm{K}$ & 2,0983 & 0,1473 & 0,3838 & 18,2920 & 93 & 2,1947 & 0,2060 & 0,4539 & 20,6828 & 126 & 0,7150 \\
\hline $\mathrm{N} / \mathrm{Ca}$ & 0,6995 & 0,0162 & 0,1271 & 18,1661 & 93 & 0,6803 & 0,0146 & 0,1209 & 17,7751 & 126 & 1,1047 \\
\hline $\mathrm{N} / \mathrm{Mg}$ & 8,3914 & 3,9038 & 1,9758 & 23,5456 & 93 & 8,1000 & 4,3627 & 2,0887 & 25,7866 & 126 & 0,8948 \\
\hline $\mathrm{N} / \mathrm{S}$ & 9,7008 & 1,6845 & 1,2979 & 13,3792 & 76 & 9,7858 & 2,0433 & 1,4294 & 14,6072 & 88 & 0,8244 \\
\hline $\mathrm{P} / \mathrm{N}$ & 0,0488 & 0,0001 & 0,0106 & 21,7256 & 93 & 0,0504 & 0,0002 & 0,0125 & 24,8264 & 126 & 0,6875 \\
\hline $\mathrm{P} / \mathrm{K}$ & 0,1029 & 0,0011 & 0,0336 & 32,6722 & 93 & 0,1103 & 0,0014 & 0,0369 & 33,4280 & 126 & 0,8309 \\
\hline $\mathrm{P} / \mathrm{Ca}$ & 0,0338 & 0,0001 & 0,0080 & 23,6849 & 93 & 0,0341 & 0,0001 & 0,0100 & 29,3896 & 126 & 0,6000 \\
\hline $\mathrm{P} / \mathrm{Mg}$ & 0,4118 & 0,0187 & 0,1368 & 33,2209 & 93 & 0,4165 & 0,0294 & 0,1716 & 41,1999 & 126 & 0,6355 \\
\hline $\mathrm{P} / \mathrm{S}$ & 0,4325 & 0,0034 & 0,0586 & 13,5546 & 76 & 0,4307 & 0,0062 & 0,0784 & 18,2110 & 88 & 0,5593 \\
\hline $\mathrm{K} / \mathrm{N}$ & 0,4921 & 0,0079 & 0,0887 & 18,0165 & 93 & 0,4733 & 0,0087 & 0,0933 & 19,7076 & 126 & 0,9034 \\
\hline $\mathbf{K} / \mathbf{P}$ & 10,4499 & 5,8142 & 2,4113 & 23,0745 & 93 & 9,9509 & 9,5709 & 3,0937 & 31,0894 & 126 & 0,6075 \\
\hline $\mathrm{K} / \mathrm{Ca}$ & 0,3478 & 0,0099 & 0,0996 & 28,6425 & 93 & 0,3201 & 0,0060 & 0,0775 & 24,2153 & 126 & $1,6506 * *$ \\
\hline $\mathrm{K} / \mathrm{Mg}$ & 4,1487 & 1,5966 & 1,2636 & 30,4572 & 93 & 3,8690 & 1,9615 & 1,4005 & 36,1986 & 126 & 0,8140 \\
\hline $\mathrm{K} / \mathrm{S}$ & 4,9010 & 0,8486 & 0,9212 & 18,7965 & 76 & 4,7298 & 1,1083 & 1,0528 & 22,2582 & 88 & 0,7657 \\
\hline $\mathrm{Ca} / \mathrm{N}$ & 1,4762 & 0,0736 & 0,2713 & 18,3804 & 93 & 1,5177 & 0,0773 & 0,2780 & 18,3189 & 126 & 0,9524 \\
\hline $\mathrm{Ca} / \mathrm{P}$ & 31,1637 & 52,7886 & 7,2656 & 23,3143 & 93 & 31,9084 & 94,8026 & 9,7367 & 30,5144 & 126 & 0,5568 \\
\hline $\mathrm{Ca} / \mathrm{K}$ & 3,1377 & 0,9947 & 0,9973 & 31,7857 & 93 & 3,3081 & 0,6394 & 0,7996 & 24,1719 & 126 & $1,5556 *$ \\
\hline $\mathrm{Ca} / \mathrm{Mg}$ & 12,1961 & 8,8301 & 2,9715 & 24,3647 & 93 & 12,0952 & 12,0056 & 3,4649 & 28,6469 & 126 & 0,7355 \\
\hline $\mathrm{Ca} / \mathrm{S}$ & 14,1432 & 13,3660 & 3,6560 & 25,8496 & 76 & 14,9368 & 13,0599 & 3,6138 & 24,1942 & 88 & 1,0234 \\
\hline $\mathrm{Mg} / \mathrm{N}$ & 0,1259 & 0,0009 & 0,0301 & 23,9327 & 93 & 0,1325 & 0,0014 & 0,0376 & 28,3657 & 126 & 0,6454 \\
\hline $\mathbf{M g} / \mathbf{P}$ & 2,6945 & 0,7554 & 0,8691 & 32,2552 & 93 & 2,8539 & 1,5126 & 1,2299 & 43,0937 & 126 & 0,4994 \\
\hline $\mathrm{Mg} / \mathrm{K}$ & 0,2663 & 0,0082 & 0,0904 & 33,9560 & 93 & 0,2925 & 0,0112 & 0,1056 & 36,1156 & 126 & 0,7330 \\
\hline $\mathrm{Mg} / \mathrm{Ca}$ & 0,0866 & 0,0004 & 0,0201 & 23,1673 & 93 & 0,0881 & 0,0004 & 0,0204 & 23,1176 & 126 & 0,9756 \\
\hline $\mathrm{Mg} / \mathrm{S}$ & 1,2666 & 0,1726 & 0,4155 & 32,8012 & 76 & 1,4110 & 0,2374 & 0,4872 & 34,5299 & 88 & 0,7272 \\
\hline $\mathbf{S} / \mathbf{N}$ & 0,1048 & 0,0002 & 0,0134 & 12,8131 & 76 & 0,1042 & 0,0002 & 0,0144 & 13,7916 & 88 & 0,8571 \\
\hline $\mathrm{S} / \mathrm{P}$ & 2,3540 & 0,0994 & 0,3153 & 13,3948 & 76 & 2,4341 & 0,5012 & 0,7080 & 29,0848 & 88 & 0,1984 \\
\hline $\mathbf{S} / \mathbf{K}$ & 0,2109 & 0,0014 & 0,0377 & 17,8538 & 76 & 0,2228 & 0,0031 & 0,0559 & 25,0799 & 88 & 0,4551 \\
\hline $\mathrm{S} / \mathrm{Ca}$ & 0,0750 & 0,0003 & 0,0176 & 23,4384 & 76 & 0,0708 & 0,0003 & 0,0166 & 23,3883 & 88 & 1,1481 \\
\hline $\mathrm{S} / \mathrm{Mg}$ & 0,8768 & 0,0829 & 0,2879 & 32,8354 & 76 & 0,7953 & 0,0751 & 0,2741 & 34,4642 & 88 & 1,1034 \\
\hline $\mathrm{N}$ & 26,1100 & 0,0451 & 2,1230 & 8,1319 & 93 & 25,6290 & 0,0477 & 2,1840 & 8,5225 & 126 & 0,9449 \\
\hline $\mathbf{P}$ & 1,2710 & 0,0008 & 0,2820 & 22,2112 & 93 & 1,2850 & 0,0011 & 0,3260 & 25,3300 & 126 & 0,7547 \\
\hline $\mathbf{K}$ & 12,7620 & 0,0387 & 1,9660 & 15,4069 & 93 & 12,0210 & 0,0355 & 1,8840 & 15,6763 & 126 & 1,0887 \\
\hline $\mathrm{Ca}$ & 38,3830 & 0,4655 & 6,8230 & 17,7761 & 93 & 38,6910 & 0,4544 & 6,7410 & 17,4223 & 126 & 1,0245 \\
\hline Mg & 3,2950 & 0,0076 & 0,8690 & 26,3863 & 93 & 3,4110 & 0,0111 & 1,0530 & 30,8656 & 126 & 0,6817 \\
\hline $\mathbf{S}$ & 2,7160 & 0,0013 & 0,3540 & 13,0365 & 76 & 2,6600 & 0,0013 & 0,3560 & 13,3973 & 88 & 0,9843 \\
\hline
\end{tabular}

$x$ : média; $e^{2}$ : variância. «: desvio padrão; c.v.: coeficiente de variação; $n .:$ número de observações;

$4^{2} /{ }^{2} / c^{2}$ razão entre variâncias das populações de alta e baixa produtividade

Os valores de $\mathrm{F}$ nos niveis de 5 e 1\% de significância são 1,47 e 1,73, respectivamente (Steel \& Torrie, 1960). 
Apêndice 9. Índices DRIS, calculados pelo procedimento proposto por Beaufils (1973), diagnose para a correção de $\mathrm{N}, \mathrm{P}$ e K, resposta líquida da produtividade, diagnose para o nutriente deficiente e a resposta da produtividade, para os dados, dos tratamentos com as doses 0 e 1 de $\mathrm{N}$ em todas as combinações de doses de $\mathrm{P}$ e $\mathrm{K}$, do ensaio de adubação $\mathrm{N}, \mathrm{P}$ e $\mathrm{K}$ conduzido em Araraquara/SP.

\begin{tabular}{|c|c|c|c|c|c|c|c|c|c|c|c|c|c|c|}
\hline \multirow[b]{2}{*}{ Trat. } & \multicolumn{6}{|c|}{ Índices DRIS } & \multirow[b]{2}{*}{$\mathrm{BN}$} & \multirow[b]{2}{*}{ Prod. } & \multicolumn{3}{|c|}{ Diagnose } & \multicolumn{3}{|c|}{ Resposta } \\
\hline & $\overline{\mathrm{N}}$ & $P$ & $\overline{\mathrm{K}}$ & $\mathrm{Ca}$ & $\mathrm{Mg}$ & $S$ & & & $\mathrm{~N}$ & $P$ & $\mathrm{~K}$ & $\overline{d\left(Y_{N}\right)}$ & $\mathrm{d}\left(\mathrm{Y}_{\mathrm{P}}\right)$ & $\mathrm{d}\left(\mathrm{Y}_{\mathrm{K}}\right)$ \\
\hline & & & & & & & & $\mathrm{tha}^{-1}$ & & & & & $-\mathrm{tha}^{-1}$ & \\
\hline 000 & -17 & 68 & 75 & -76 & -77 & 28 & 57 & 31,33 & $\mathrm{~V}^{+}$ & $\mathrm{V}^{-}$ & $\mathrm{F}^{-}$ & 3,64 & 0,00 & $-2,18$ \\
\hline 001 & -16 & 67 & 105 & $-63-$ & -119 & 26 & 66 & 33,51 & $\mathrm{~V}^{+}$ & $\mathrm{F}^{-}$ & $\mathrm{F}^{-}$ & 3,36 & $-0,44$ & $-1,02$ \\
\hline 002 & -11 & 119 & 138 & $-66-$ & -199 & 19 & 92 & 34,53 & $\mathrm{~V}^{+}$ & $\mathrm{F}^{-}$ & $\mathrm{V}^{-}$ & 2,92 & $-0,88$ & 0,14 \\
\hline 003 & 13 & 136 & 182 & $-69-$ & -293 & 31 & 121 & 34,39 & $\mathrm{~F}^{-}$ & $\mathrm{F}^{-}$ & - & $-2,48$ & $-1,16$ & 0,00 \\
\hline 010 & -15 & 107 & 69 & -86 & -86 & 12 & 63 & 31,33 & $\mathrm{~V}^{+}$ & $\mathrm{V}^{-}$ & $\mathrm{F}^{-}$ & 3,35 & 0,00 & $-2,62$ \\
\hline 011 & -5 & 118 & 108 & $-67-$ & -146 & -9 & 76 & 33,95 & $\mathrm{~V}^{+}$ & $\mathrm{F}^{-}$ & $\mathrm{F}^{-}$ & 3,06 & $-0,44$ & $-1,46$ \\
\hline 012 & 0 & 124 & 145 & $-66-$ & -197 & -6 & 90 & 35,41 & $\mathrm{~F}^{-}$ & $F^{-}$ & $\mathrm{F}^{-}$ & $-2,62$ & $-0,73$ & $-0,14$ \\
\hline 013 & 22 & 142 & 190 & $-70-$ & -290 & 6 & 120 & 35,55 & $F^{-}$ & $\mathrm{F}^{-}$ & - & $-2,19$ & $-1,17$ & 0,00 \\
\hline 020 & -4 & 113 & 73 & -87 & -84 & -11 & 62 & 31,33 & $\mathrm{~V}^{+}$ & $\mathrm{V}^{-}$ & $\mathrm{F}^{-}$ & 3,06 & 0,15 & $-3,06$ \\
\hline 021 & -10 & 115 & 109 & $-76-$ & -125 & -12 & 75 & 34,39 & $\mathrm{~V}^{+}$ & $F^{-}$ & $\mathrm{F}^{-}$ & 2,62 & $-0,14$ & $-1,75$ \\
\hline 022 & -2 & 128 & 150 & $-74-$ & -168 & -33 & 93 & 36,14 & $\mathrm{~V}^{+}$ & $F^{-}$ & $F^{-}$ & 2,33 & $-0,58$ & $-0,58$ \\
\hline 023 & 12 & 145 & 199 & $-81-$ & -251 & -23 & 119 & 36,72 & $\mathrm{~F}^{-}$ & $F^{-}$ & - & $-1,89$ & $-1,02$ & 0,00 \\
\hline 030 & -30 & 145 & 62 & 112 & -60 & -5 & 69 & 31,18 & $\mathrm{~V}^{+}$ & - & $\mathrm{F}^{-}$ & 2,63 & 0,00 & $-3,35$ \\
\hline 031 & -25 & 110 & 108 & -90 & -88 & -15 & 73 & 34,53 & $\mathrm{~V}^{+}$ & - & $\mathrm{F}^{-}$ & 2,34 & 0,00 & $-2,19$ \\
\hline 032 & -20 & 127 & 157 & $-87=$ & -144 & -34 & 95 & 36 & $\mathrm{~V}^{+}$ & - & $\mathrm{F}^{-}$ & 2,04 & 0 , & $-1,02$ \\
\hline 033 & -14 & 146 & 206 & $-98-$ & -187 & -53 & 117 & 37,74 & $\mathrm{~V}^{+}$ & - & - & 1,60 & 0,00 & 0,00 \\
\hline 100 & 32 & 28 & 74 & -30 & -92 & -12 & 45 & 34,97 & $\mathrm{~F}^{-}$ & $\mathrm{V}^{-}$ & $\mathrm{F}^{-}$ & $-2,33$ & 0,29 & $-1,90$ \\
\hline 101 & 22 & 69 & 94 & $-33-$ & -123 & -30 & 62 & 36,87 & $F^{-}$ & $\mathrm{F}^{-}$ & $\mathrm{F}^{-}$ & $-1,89$ & $-0,14$ & $-0,58$ \\
\hline 102 & 29 & 72 & 133 & $-34-$ & -171 & -30 & 78 & 37,45 & $\mathrm{~F}^{-}$ & $\mathrm{F}^{-}$ & $\mathrm{V}^{-}$ & $-1,46$ & $-0,58$ & 0,58 \\
\hline 103 & 42 & 80 & 173 & $-45-$ & -225 & -25 & 98 & 36,87 & $\mathrm{~F}^{-}$ & $F^{-}$ & - & $-1,16$ & $-0,87$ & 0,00 \\
\hline 110 & 34 & 70 & 61 & -40 & -99 & -26 & 55 & 34,68 & $\mathrm{~F}^{-}$ & $\mathrm{V}^{-}$ & $\mathrm{F}^{-}$ & $-2,04$ & 0,29 & $-2,33$ \\
\hline 111 & 35 & 72 & 98 & $-33-$ & -144 & -28 & 68 & 37,01 & $\mathrm{~F}^{-}$ & $\mathrm{V}^{-}$ & $\mathrm{F}^{-}$ & $-1,46$ & 0,00 & $-1,02$ \\
\hline 112 & 35 & 72 & 133 & $-40-$ & -171 & -30 & 80 & 38,03 & $\mathrm{~F}^{-}$ & $F^{-}$ & $\mathrm{V}^{-}$ & $-1,17$ & $-0,44$ & 0,29 \\
\hline 113 & 52 & 88 & 179 & $-48-$ & -221 & -50 & 106 & 37,74 & $\mathrm{~F}^{-}$ & $\mathrm{F}^{-}$ & - & $-0,87$ & $-0,87$ & 0,00 \\
\hline 120 & 40 & 71 & 62 & -47 & -99 & -27 & 58 & 34,39 & $\mathrm{~F}^{-}$ & $\mathrm{V}^{-}$ & $\mathrm{F}^{-}$ & $-1,60$ & 0,58 & $-2,62$ \\
\hline 121 & 37 & 76 & 103 & $-40-$ & -121 & -55 & 72 & 37,01 & $\mathrm{~F}^{-}$ & $\mathrm{V}^{-}$ & $\mathrm{F}^{-}$ & $-1,17$ & 0,14 & $-1,46$ \\
\hline 122 & 38 & 82 & 140 & $-43-$ & -165 & -52 & 87 & 38,47 & $\mathrm{~F}^{*}$ & $F^{-}$ & $\mathrm{F}^{-}$ & $-0,87$ & $-0,29$ & $-0,14$ \\
\hline 123 & 43 & 91 & 187 & $-57-$ & -217 & -47 & 107 & & $F^{-}$ & $F^{-}$ & - & $-0,59$ & $-0,73$ & 0,00 \\
\hline 130 & 30 & 70 & 64 & -57 & -80 & -27 & 55 & 33,81 & $F^{-}$ & - & $\mathrm{F}^{-}$ & $-1,31$ & 0,00 & $-3,06$ \\
\hline 131 & 25 & 76 & 103 & -52 & -99 & -54 & 68 & 36,87 & $\mathrm{~F}^{-}$ & - & $\mathrm{F}^{-}$ & $-1,02$ & 0,00 & $-1,89$ \\
\hline 132 & 22 & 82 & 144 & $-58-$ & -139 & -51 & 83 & 38,76 & $F^{-}$ & - & $\mathrm{F}^{-}$ & $-0,58$ & 0,00 & $-0,58$ \\
\hline 133 & 24 & 95 & 191 & $-76-$ & -159 & -76 & 104 & 39,34 & $\mathrm{~F}^{-}$ & - & - & $-0,29$ & 0,00 & 0,00 \\
\hline
\end{tabular}


Apêndice 10. Índices DRIS, calculados pelo procedimento proposto por Beaufils (1973), diagnose para a correção de N, P e K, resposta líquida da produtividade, diagnose para o nutriente deficiente e a resposta da produtividade, para os dados, dos tratamentos com as doses 2 e 3 de $\mathrm{N}$ em todas as combinações de doses de $\mathrm{P}$ e $\mathrm{K}$, do ensaio de adubação $\mathrm{N}, \mathrm{P}$ e $\mathrm{K}$ conduzido em Araraquara/SP.

\begin{tabular}{|c|c|c|c|c|c|c|c|c|c|c|c|c|c|c|}
\hline \multirow[b]{2}{*}{ Trat. } & \multicolumn{6}{|c|}{ Indices DRIS } & \multirow[b]{2}{*}{$\mathrm{BN}$} & \multirow[b]{2}{*}{ Prod. } & \multicolumn{3}{|c|}{ Diagnose } & \multicolumn{3}{|c|}{ Resposta } \\
\hline & $\overline{\mathrm{N}}$ & $\mathrm{P}$ & $\mathrm{K}$ & $\mathrm{Ca}$ & $\mathrm{Mg}$ & $\bar{S}$ & & & $\mathrm{~N}$ & $\mathrm{P}$ & $\mathrm{K}$ & $d\left(Y_{N}\right)$ & $\mathrm{d}\left(\mathrm{Y}_{\mathrm{P}}\right)$ & $\mathrm{d}\left(\mathrm{Y}_{\mathrm{K}}\right)$ \\
\hline & & & & & & & & $\mathrm{tha}^{-1}$ & & & & & $-t h a^{-1}$ & \\
\hline 200 & 48 & 28 & 55 & -16 & -75 & -40 & 44 & 37,30 & $\mathrm{~F}^{-}$ & $\mathrm{V}^{-}$ & $\mathrm{F}^{-}$ & $-0,88$ & 0,58 & $-1,46$ \\
\hline 201 & 37 & 69 & 83 & -23 & -105 & -60 & 63 & 38,76 & $\mathrm{~F}^{-}$ & $\mathrm{V}^{-}$ & $\mathrm{F}^{-}$ & $-0,44$ & 0,29 & $-0,15$ \\
\hline 202 & 33 & 61 & 112 & -35 & -131 & -40 & 69 & 38,91 & $\mathrm{~F}^{-}$ & $F^{-}$ & $\mathrm{V}^{-}$ & $-0,14$ & $-0,29$ & 0,88 \\
\hline 203 & 30 & 108 & 138 & -64 & -160 & -53 & 92 & 38,03 & $\mathrm{~V}^{-}$ & $F^{-}$ & - & 0,29 & $-0,58$ & 0,00 \\
\hline 210 & 56 & 74 & 46 & -25 & -99 & -53 & 59 & 36,72 & $\mathrm{~F}^{-}$ & $\mathrm{V}^{-}$ & $\mathrm{F}^{-}$ & $-0,44$ & 0,73 & $-1,75$ \\
\hline 211 & 51 & 72 & 83 & -25 & -123 & -58 & 69 & 38,47 & $\mathrm{~F}^{-}$ & $\mathrm{V}^{*}$ & $\mathrm{~F}^{-}$ & $-0,29$ & 0,29 & $-0,73$ \\
\hline 212 & 49 & 73 & 118 & -34 & -147 & -59 & 80 & 39,20 & $\mathrm{~V}^{-}$ & $\mathrm{F}^{-}$ & $\mathrm{V}^{-}$ & 0,29 & $-0,14$ & 0,59 \\
\hline 213 & 53 & 77 & 157 & -56 & -171 & -59 & 96 & 38,61 & $\mathrm{~V}^{*}$ & $F^{-}$ & - & 0,58 & $-0,59$ & 0,00 \\
\hline 220 & 59 & 76 & 44 & -31 & -97 & -51 & 60 & 35,99 & $\mathrm{~F}^{-}$ & $\mathrm{V}^{-}$ & $\mathrm{F}^{-}$ & $-0,15$ & 0,87 & $-2,19$ \\
\hline 221 & 51 & 75 & 81 & -32 & -121 & -55 & 69 & 38,18 & $\mathrm{~V}^{-}$ & $\mathrm{V}^{-}$ & $\mathrm{F}^{-}$ & 0,15 & 0,29 & $-1,16$ \\
\hline 222 & 46 & 76 & 121 & -43 & -144 & -56 & 81 & 39,34 & $\mathrm{~V}^{-}$ & $\mathrm{V}^{-}$ & $\mathrm{V}^{-}$ & 0,58 & 0,00 & 0,14 \\
\hline 223 & 53 & 89 & 167 & -63 & -164 & -81 & 103 & 39,20 & $\mathrm{~V}^{-}$ & $F^{-}$ & - & 0,88 & $-0,43$ & 0,00 \\
\hline 230 & 55 & 80 & 47 & -40 & -94 & -47 & 61 & 35,12 & $\mathrm{~V}^{-}$ & - & $\mathrm{F}^{-}$ & 0,00 & 0,00 & $-2,77$ \\
\hline 231 & 40 & 76 & 81 & -46 & -98 & -53 & 66 & 37,89 & $\mathrm{~V}^{-}$ & - & $\mathrm{F}^{-}$ & 44 & 0,00 & $-1,45$ \\
\hline 232 & 37 & 86 & 128 & -55 & -116 & -79 & 84 & 39,34 & $\mathrm{~V}^{-}$ & - & $\mathrm{F}^{-}$ & 0,87 & 0,00 & $-0,29$ \\
\hline 233 & 33 & 91 & 173 & -82 & -136 & -78 & 99 & 39,63 & $\mathrm{~V}^{-}$ & - & - & 1,16 & 0,00 & 0,00 \\
\hline 300 & 35 & 64 & 26 & -28 & -66 & -30 & 42 & 38,18 & - & $\mathrm{V}^{-}$ & $\mathrm{F}^{-}$ & 0,00 & 1,02 & $-1,02$ \\
\hline 301 & 18 & 100 & 52 & -41 & -78 & -51 & 57 & 39,20 & - & $\mathrm{V}^{-}$ & $\mathrm{V}^{-}$ & 0,00 & 0,44 & 0,15 \\
\hline 302 & 16 & 98 & 81 & -58 & -81 & -57 & 65 & 39,05 & - & $\mathrm{V}^{-}$ & $\mathrm{V}^{-}$ & 0,00 & 0,14 & 1,31 \\
\hline 303 & 3 & 132 & 106 & -99 & -91 & -50 & 80 & 37,74 & - & $F^{-}$ & - & 0,00 & $-0,29$ & 0,00 \\
\hline 310 & 57 & 75 & 25 & -28 & -79 & -51 & 53 & 37,16 & - & $\mathrm{V}^{-}$ & $\mathrm{F}^{-}$ & 0,00 & 1,02 & $-1,60$ \\
\hline 311 & 33 & 105 & 48 & -46 & -92 & -48 & 62 & 38,76 & - & $\mathrm{V}^{-}$ & $F^{-}$ & 0,00 & 0,73 & $-0,15$ \\
\hline 312 & 26 & 103 & 82 & -64 & -95 & -52 & 70 & 38,91 & - & $\mathrm{V}^{-}$ & $\mathrm{V}^{-}$ & 0,00 & 0,15 & 0,88 \\
\hline 313 & 22 & 104 & 119 & -95 & -96 & -55 & 82 & 38,03 & - & $F^{-}$ & - & 0,00 & $-0,29$ & 0,00 \\
\hline 320 & 50 & 117 & 15 & -44 & -102 & -36 & 61 & 36,14 & - & $\mathrm{V}^{-}$ & $F^{-}$ & 0,00 & 1,02 & $-1,89$ \\
\hline 321 & 42 & 120 & 55 & $-49-$ & -103 & -65 & 72 & 38,03 & - & $\mathrm{V}^{-}$ & $\mathrm{F}^{-}$ & 0,00 & 0,58 & $-0,73$ \\
\hline 322 & 29 & 115 & 87 & -71 & -89 & -72 & 77 & 38,76 & - & $\mathrm{V}^{-}$ & $\mathrm{V}^{-}$ & 0,00 & 0,29 & 0,44 \\
\hline 323 & 28 & 121 & 130 & $-101-$ & -107 & -71 & 93 & 38,32 & - & $F^{*}$ & - & 0,00 & $-0,15$ & 0,00 \\
\hline 330 & 41 & 119 & 12 & -58 & -81 & -33 & 57 & 35,12 & - & - & $\mathrm{F}^{-}$ & 0,00 & 0,00 & $-2,33$ \\
\hline 331 & 24 & 115 & 51 & -67 & -85 & -38 & 63 & 37,45 & - & - & $\mathrm{F}^{-}$ & 0,00 & 0,00 & $-1,02$ \\
\hline 332 & 19 & 122 & 93 & -85 & -84 & -65 & 78 & 38,47 & - & - & $\mathrm{V}^{-}$ & 0,00 & 0,00 & 0,00 \\
\hline 333 & 9 & 126 & 134 & -121 & -83 & -65 & 90 & 38,47 & - & - & - & 0,00 & 0,00 & 0,00 \\
\hline
\end{tabular}


Apêndice 11. Índices DRIS, calculados pelo procedimento proposto por Beaufils (1973), diagnose para a correção de $\mathrm{N}, \mathrm{P}$ e K, resposta líquida da produtividade, diagnose para o nutriente deficiente e a resposta da produtividade, para os dados, dos tratamentos com as doses 0 e 1 de $\mathrm{N}$ em todas as combinações de doses de $\mathrm{P}$ e $\mathrm{K}$, do ensaio de adubação $\mathrm{N}, \mathrm{P}$ e $\mathrm{K}$ conduzido em Botucatu/SP.

\begin{tabular}{|c|c|c|c|c|c|c|c|c|c|c|c|c|c|}
\hline \multirow[b]{2}{*}{ Trat. } & \multicolumn{5}{|c|}{ Índices DRIS } & \multirow[b]{2}{*}{$\mathrm{BN}$} & \multirow[b]{2}{*}{ Prod. } & \multicolumn{3}{|c|}{ Diagnose } & \multicolumn{3}{|c|}{ Resposta } \\
\hline & $\mathrm{N}$ & $\mathrm{K}$ & $\mathrm{Ca}$ & $\mathrm{Mg}$ & $\mathbf{S}$ & & & $\mathrm{N}$ & $\mathrm{P}$ & $\mathrm{K}$ & $\mathrm{d}\left(\mathrm{Y}_{\mathrm{N}}\right)$ & $d\left(Y_{P}\right)$ & $\mathrm{d}\left(\mathrm{Y}_{\mathrm{K}}\right)$ \\
\hline & & & & & & & $\mathrm{tha}^{-1}$ & & & & & $\mathrm{tha}^{-1}$ & \\
\hline 000 & -118 & $203-728$ & 154 & 477 & 11 & 282 & 36,04 & $\mathrm{~V}^{+}$ & $\mathrm{F}^{-}$ & $\mathrm{V}^{+}$ & 4,08 & $-0,76$ & 4,93 \\
\hline 001 & -161 & $118-196$ & 45 & 276 & -82 & 146 & 40,97 & $\mathrm{~V}^{+}$ & $\mathrm{F}^{-}$ & $\mathrm{V}^{+}$ & 4,42 & $-0,76$ & 2,29 \\
\hline 002 & -186 & $99 \quad 38$ & -56 & 164 & -59 & 100 & 43,26 & $\mathrm{~V}^{+}$ & $\mathrm{F}^{-}$ & $\mathrm{V}^{+}$ & 4,77 & $-0,69$ & 0,42 \\
\hline 003 & -209 & 77217 & -189 & 90 & 13 & 133 & 42,84 & $\mathrm{~V}^{+}$ & $\mathrm{F}^{-}$ & - & 5,10 & $-0,68$ & 0,00 \\
\hline 010 & -202 & $242-822$ & 204 & 518 & 61 & 342 & 36,80 & $\mathrm{~V}^{+}$ & $\mathrm{F}^{-}$ & $\mathrm{V}^{+}$ & 4,51 & $-1,71$ & 4,93 \\
\hline 011 & -251 & $136-253$ & 79 & 302 & -13 & 172 & 41,73 & $\mathrm{~V}^{+}$ & $\mathrm{F}^{-}$ & $\mathrm{V}^{+}$ & 4,85 & $-1,71$ & 2,22 \\
\hline 012 & -261 & 66 & -8 & 182 & 15 & 90 & 43,95 & $\mathrm{~V}^{+}$ & $\mathrm{F}^{-}$ & $\mathrm{V}^{-}$ & 5,27 & $-1,70$ & 0,43 \\
\hline 013 & -284 & $96173-$ & -128 & 105 & 38 & 137 & 43,52 & $\mathrm{~V}^{+}$ & $\mathrm{F}^{-}$ & - & 5,52 & $-1,61$ & 0,00 \\
\hline 020 & -245 & $232-925$ & 244 & 560 & 133 & 390 & 38,51 & $\mathrm{~V}^{+}$ & $\mathrm{F}^{-}$ & $\mathrm{V}^{+}$ & 5,01 & $-2,72$ & 4,93 \\
\hline 021 & -291 & $126-302$ & 116 & 337 & 14 & 198 & 43,44 & $\mathrm{~V}^{+}$ & $\mathrm{F}^{-}$ & $\mathrm{V}^{+}$ & 5,35 & $-2,72$ & 2,21 \\
\hline 022 & -304 & $100-36$ & 16 & 205 & 20 & 114 & 45,65 & $\mathrm{~V}^{+}$ & $\mathrm{F}^{-}$ & $\mathrm{F}^{+}$ & 5,69 & $-2,72$ & $-0,52$ \\
\hline 023 & -309 & $81 \quad 138$ & -84 & 119 & 55 & 131 & 45,13 & $\mathrm{~V}^{+}$ & $\mathrm{F}^{-}$ & - & 6,04 & $-2,72$ & 0,00 \\
\hline 030 & -225 & $267-1154$ & 306 & 635 & 171 & 460 & 41,23 & $\mathrm{~V}^{+}$ & - & $\mathrm{V}^{+}$ & 5,43 & 0,00 & 4,93 \\
\hline 031 & -297 & $125-385$ & 140 & 368 & 50 & 228 & 46,16 & $\mathrm{~V}^{+}$ & - & $\mathrm{V}^{+}$ & 5,78 & 0,00 & 2,21 \\
\hline 032 & -305 & $\begin{array}{ll}97 & -75\end{array}$ & 37 & 229 & 18 & 127 & 48 & $\mathrm{~V}^{+}$ & - & $\mathrm{F}^{+}$ & 6,12 & 0,00 & $-0,52$ \\
\hline 033 & -301 & $73 \quad 105$ & -61 & 136 & 47 & 121 & 47,85 & $\mathrm{~V}^{+}$ & - & - & 6,46 & 0,00 & 0,00 \\
\hline 100 & 14 & $267-1161$ & 242 & 614 & 22 & 387 & 40,12 & $F^{-}$ & $F^{-}$ & $\mathrm{V}^{+}$ & $-1,28$ & $-1,19$ & 5,27 \\
\hline 101 & -84 & $123-395$ & 81 & 356 & -81 & 187 & 45,39 & $\mathrm{~V}^{+}$ & $\mathrm{F}^{-}$ & $\mathrm{V}^{+}$ & 1,70 & $-1,19$ & 2,64 \\
\hline 102 & -114 & $\begin{array}{ll}86 & -87\end{array}$ & -32 & 220 & -74 & 102 & 48,03 & $\mathrm{~V}^{+}$ & $\mathrm{F}^{-}$ & $\mathrm{F}^{+}$ & 2,03 & $-1,19$ & $-0,09$ \\
\hline 103 & -135 & $10475-$ & -156 & 133 & -20 & 104 & 47,94 & $\mathrm{~V}^{+}$ & $\mathrm{F}^{-}$ & - & 2,38 & $-1,10$ & 0,00 \\
\hline 110 & -57 & $308-1239$ & 288 & 621 & 79 & 432 & 41,31 & $\mathrm{~V}^{+}$ & $\mathrm{F}^{-}$ & $\mathrm{V}^{+}$ & 1,78 & $-2,21$ & 5,27 \\
\hline 111 & -153 & $154-440$ & 119 & 358 & -38 & 210 & 46,58 & $\mathrm{~V}^{+}$ & $\mathrm{F}^{-}$ & $\mathrm{V}^{+}$ & 2,13 & $-2,21$ & 2,64 \\
\hline 112 & -176 & $111-122$ & 5 & 222 & -40 & 113 & 49,22 & $\mathrm{~V}^{+}$ & $\mathrm{F}^{-}$ & $\mathrm{F}^{+}$ & 2,37 & $-2,12$ & $-0,18$ \\
\hline 113 & -178 & $82 \quad 49$ & -99 & 153 & -8 & 95 & 49,04 & $\mathrm{~V}^{+}$ & $F^{-}$ & - & 2,81 & $-2,13$ & 0,00 \\
\hline 120 & -76 & $314-1376$ & 341 & 666 & 131 & 484 & 43,52 & $\mathrm{~V}^{+}$ & $\mathrm{F}^{-}$ & $\mathrm{V}^{+}$ & 2,21 & $-3,14$ & 5,27 \\
\hline 121 & -188 & $202-521$ & 147 & 378 & -19 & 243 & 48,79 & $\mathrm{~V}^{+}$ & $F^{-}$ & $\mathrm{V}^{+}$ & 2,55 & $-3,15$ & 2,55 \\
\hline 122 & -192 & $106-151$ & 38 & 243 & -45 & 129 & 51,34 & $\mathrm{~V}^{+}$ & $\mathrm{F}^{-}$ & $\mathrm{F}^{+}$ & 2,89 & $-3,15$ & $-0,17$ \\
\hline 123 & -209 & 11320 & -72 & 149 & -2 & 94 & 51,17 & $\mathrm{~V}^{+}$ & $\mathrm{F}^{-}$ & - & 3,32 & $-3,14$ & 0,00 \\
\hline 130 & -32 & $446-1726$ & 416 & 769 & 128 & 586 & 46,66 & $\mathrm{~V}^{+}$ & - & $\mathrm{V}^{+}$ & 2,64 & 0,00 & 5,28 \\
\hline 131 & -164 & $223-609$ & 183 & 410 & -43 & 272 & 51,94 & $\mathrm{~V}^{+}$ & - & $\mathrm{V}^{+}$ & 2,97 & 0,00 & 2,55 \\
\hline 132 & -196 & $157-209$ & 53 & 253 & -57 & 154 & 54,49 & $\mathrm{~V}^{+}$ & - & $\mathrm{F}^{+}$ & 3,31 & 0,00 & $-0,18$ \\
\hline 133 & -191 & $\begin{array}{ll}117 & -7 \\
\end{array}$ & -51 & 161 & -29 & 93 & 54,31 & $\mathrm{~V}^{+}$ & - & - & 3,66 & 0,00 & 0,00 \\
\hline
\end{tabular}


Apêndice 12. Índices DRIS, calculados pelo procedimento proposto por Beaufils (1973), diagnose para a correção de N, P e K, resposta líquida da produtividade, diagnose para o nutriente deficiente e a resposta da produtividade, para os dados, dos tratamentos com as doses 2 e 3 de $\mathrm{N}$ em todas as combinações de doses de P e K, do ensaio de adubação N, P e K conduzido em Botucatu/SP.

\begin{tabular}{|c|c|c|c|c|c|c|c|c|c|c|c|c|}
\hline \multirow[b]{2}{*}{ Trat. } & \multicolumn{4}{|c|}{ Índices DRIS } & \multirow[b]{2}{*}{$\mathrm{BN}$} & \multirow[b]{2}{*}{ Prod. } & \multicolumn{3}{|c|}{ Diagnose } & \multicolumn{3}{|c|}{ Resposta } \\
\hline & $\bar{N}$ & $\mathrm{~K}$ & $\mathrm{Ca}$ & $\mathrm{Mg}$ & & & $\mathrm{N}$ & $P$ & $\bar{K}$ & $d\left(Y_{N}\right)$ & $\mathrm{d}\left(\mathrm{Y}_{\mathrm{P}}\right)$ & $\mathrm{d}\left(\mathrm{Y}_{\mathrm{K}}\right)$ \\
\hline & & & & & & $\mathrm{tha}^{-1}$ & & & & & $t h a^{-1}$ & \\
\hline 200 & 116 & $324-1513$ & 318 & $\begin{array}{ll}735 & 19\end{array}$ & 504 & 41,40 & $\mathrm{~V}^{-}$ & $\mathrm{F}^{-}$ & $\mathrm{V}^{+}$ & 1,45 & $-1,69$ & 5,69 \\
\hline 201 & -16 & $146-576$ & 118 & $440-111$ & 235 & 47,09 & $\mathrm{~F}^{+}$ & $\mathrm{F}^{-}$ & $\mathrm{V}^{+}$ & $-1,19$ & $-1,62$ & 2,97 \\
\hline 202 & -74 & $143-207$ & -19 & $286-129$ & 143 & 50,06 & $\mathrm{~F}^{+}$ & $\mathrm{F}^{-}$ & $\mathrm{V}^{+}$ & $-0,84$ & $-1,53$ & 0,26 \\
\hline 203 & -77 & $105-16$ & -130 & $206 \quad-88$ & 104 & 50,32 & $\mathrm{~F}^{+}$ & $\mathrm{F}^{-}$ & - & $-0,51$ & $-1,53$ & 0,00 \\
\hline 210 & 41 & $366-1522$ & 353 & $734 \quad 29$ & 508 & 43,09 & $\mathrm{~V}^{-}$ & $\mathrm{F}^{-}$ & $\mathrm{V}^{+}$ & 1,10 & $-2,64$ & 5,62 \\
\hline 211 & -82 & $244-636$ & 150 & $440-116$ & 278 & 48,71 & $\mathrm{~F}^{+}$ & $\mathrm{F}^{-}$ & $\mathrm{V}^{+}$ & $-0,68$ & $-2,63$ & 2,88 \\
\hline 212 & -121 & $179-240$ & 21 & $280-118$ & 160 & 51,59 & $\mathrm{~F}^{+}$ & $\mathrm{F}^{-}$ & $\mathrm{V}^{+}$ & $-0,33$ & $-2,64$ & 0,26 \\
\hline 213 & -127 & $125 \quad-44$ & -90 & $\begin{array}{ll}193 & -57\end{array}$ & 106 & 51,85 & $\mathrm{~F}^{+}$ & $\mathrm{F}^{-}$ & - & 0,00 & $-2,64$ & 0,00 \\
\hline 220 & 36 & $458-1706$ & 409 & $773 \quad 30$ & 569 & 45,73 & $\mathrm{~V}^{-}$ & $\mathrm{F}^{-}$ & $\mathrm{V}^{+}$ & 0,60 & $-3,57$ & 5,61 \\
\hline 221 & -90 & $251-685$ & 192 & $445-113$ & 296 & 51, & $\mathrm{~F}^{+}$ & $\mathrm{F}^{-}$ & $\mathrm{V}^{+}$ & $-0,25$ & $-3,57$ & 2,89 \\
\hline 222 & -129 & $178-263$ & 56 & $277-120$ & 171 & 54,23 & $\mathrm{~V}^{+}$ & $\mathrm{F}^{-}$ & $\mathrm{V}^{+}$ & 0,08 & $-3,57$ & 0,26 \\
\hline 223 & -135 & $174-65$ & -60 & $186-100$ & 120 & 54,49 & $\mathrm{~V}^{+}$ & $\mathrm{F}^{-}$ & - & 0,42 & $-3,48$ & 0,00 \\
\hline 230 & 107 & $538-2038$ & 497 & $861 \quad 35$ & 679 & 49,30 & $\mathrm{~V}^{-}$ & - & $\mathrm{V}^{+}$ & 0,08 & 0,00 & 5,61 \\
\hline 231 & -70 & $338-794$ & 223 & $463-160$ & 341 & 54,91 & $\mathrm{~V}^{+}$ & - & $\mathrm{V}^{+}$ & 0,25 & 0,00 & 2,89 \\
\hline 232 & -109 & $258-312$ & 79 & $289-205$ & 209 & 57,80 & $\mathrm{~V}^{+}$ & - & $\mathrm{V}^{+}$ & 0,60 & 0,00 & 0,17 \\
\hline 233 & -117 & $184 \quad-88$ & -42 & $190-128$ & 125 & 57 & $\mathrm{~V}^{+}$ & - & - & 4 & 0 & 0,00 \\
\hline 300 & 160 & $363-1571$ & 351 & $843-146$ & 572 & 39,95 & - & $F^{-}$ & $\mathrm{V}^{+}$ & 0,00 & $-2,04$ & 5,95 \\
\hline 301 & 27 & $250-674$ & 146 & $528-277$ & 317 & 45,90 & - & $\mathrm{F}^{-}$ & $\mathrm{V}^{+}$ & 0,00 & $-2,13$ & 3,32 \\
\hline 302 & -20 & $181-285$ & 9 & $367-251$ & 186 & 49,22 & - & $F^{-}$ & $\mathrm{V}^{+}$ & 0,00 & $-2,04$ & 0,59 \\
\hline 303 & -51 & $166-87-$ & -126 & $271-174$ & 146 & 49,81 & - & $\mathrm{F}^{-}$ & - & 0,00 & $-2,04$ & 0,00 \\
\hline 310 & 76 & $395-1497$ & 370 & $787-131$ & 543 & 41,99 & - & $\mathrm{F}^{-}$ & $\mathrm{V}^{+}$ & 0,00 & $-3,14$ & 6,04 \\
\hline 311 & -28 & $288-688$ & 180 & $500-252$ & 323 & 48 , & - & $\mathrm{F}^{-}$ & $\mathrm{V}^{+}$ & 0,00 & $-3,06$ & 3,23 \\
\hline 312 & -69 & $216-300$ & 44 & $343-233$ & 201 & 51,26 & - & $\mathrm{F}^{-}$ & $\mathrm{V}^{+}$ & 0,00 & $-3,05$ & 0,59 \\
\hline 313 & -90 & $195-105$ & -86 & $249-163$ & 148 & 51,85 & - & $F^{-}$ & - & 0,00 & $-3,06$ & 0,00 \\
\hline 320 & 72 & $492-1583$ & 419 & $803-203$ & 595 & 45,13 & - & $\mathrm{F}^{-}$ & $\mathrm{V}^{+}$ & 0,00 & $-4,09$ & 5,96 \\
\hline 321 & -34 & $374-748$ & 221 & $511-325$ & 369 & 51,09 & - & $\mathrm{F}^{-}$ & $\mathrm{V}^{+}$ & 0,00 & $-4,07$ & 3,22 \\
\hline 322 & -72 & $284-331$ & 75 & $343-300$ & 234 & 54,31 & - & $\mathrm{F}^{-}$ & $\mathrm{V}^{+}$ & 0,00 & $-4,09$ & 0,60 \\
\hline 323 & -93 & $253-127$ & -56 & $234-212$ & 163 & 54,91 & - & $\mathrm{F}^{*}$ & - & 0,00 & $-4,00$ & 0,00 \\
\hline 330 & 138 & $651-1801$ & 487 & $857-332$ & 711 & 49,22 & - & - & $\mathrm{V}^{+}$ & 0,00 & 0,00 & 5,94 \\
\hline 331 & 10 & $495-824$ & 261 & $529-470$ & 432 & 55,16 & - & - & $\mathrm{V}^{+}$ & 0,00 & 0,00 & 3,24 \\
\hline 332 & -36 & $387-371$ & 105 & $337-421$ & 276 & 58,40 & - & - & $\mathrm{V}^{+}$ & 0,00 & 0,00 & 0,51 \\
\hline 333 & -56 & $285-146$ & -30 & $236-288$ & 174 & 58,91 & - & - & - & 0,00 & 0,00 & 0,00 \\
\hline
\end{tabular}


Apêndice 13. Índices DRIS, calculados pelo procedimento proposto por Beaufils (1973), diagnose para a correção de $\mathrm{N}, \mathrm{P}$ e K, resposta líquida da produtividade, diagnose para o nutriente deficiente e a resposta da produtividade, para os dados, dos tratamentos com as doses 0 e 1 de $\mathrm{N}$ em todas as combinações de doses de $\mathrm{P}$ e $\mathrm{K}$, do ensaio de adubação $\mathrm{N}, \mathrm{P}$ e $\mathrm{K}$ conduzido em Matão/SP.

\begin{tabular}{|c|c|c|c|c|c|c|c|c|c|c|c|c|c|c|}
\hline \multirow[b]{2}{*}{ Trat. } & \multicolumn{6}{|c|}{ Índices DRIS } & \multirow[b]{2}{*}{$\mathrm{BN}$} & \multirow[b]{2}{*}{ Prod. } & \multicolumn{3}{|c|}{ Diagnose } & \multicolumn{3}{|c|}{ Resposta } \\
\hline & $\overline{\mathrm{N}}$ & $\mathrm{P}$ & $\mathrm{K}$ & $\mathrm{Ca}$ & $\mathrm{Mg}$ & $S$ & & & $\mathrm{~N}$ & $P$ & $\mathrm{~K}$ & $\mathrm{~d}\left(\mathrm{Y}_{\mathrm{N}}\right)$ & $\mathrm{d}\left(\mathrm{Y}_{\mathrm{P}}\right)$ & $\mathrm{d}\left(\mathrm{Y}_{\mathrm{K}}\right)$ \\
\hline & & & & & & & & $\mathrm{tha}^{-1}$ & & & & & $t h a^{-1}$ & \\
\hline 000 & -91 & -20 & 53 & 4 & 112 & -58 & 56 & 34,77 & $\mathrm{~V}^{+}$ & $\mathrm{V}^{+}$ & $\mathrm{V}^{-}$ & 2,21 & 4,75 & 1,54 \\
\hline 001 & -107 & 4 & 121 & -34 & 83 & -67 & 69 & 33,23 & $\mathrm{~V}^{+}$ & $\mathrm{F}^{-}$ & $\mathrm{F}^{-}$ & 2,04 & $-4,34$ & $-0,35$ \\
\hline 002 & -101 & 7 & 198 & -58 & 51 & -97 & 85 & 33,58 & $\mathrm{~V}^{+}$ & $\mathrm{F}^{-}$ & $\mathrm{F}^{-}$ & 1,95 & $-3,90$ & $-2,21$ \\
\hline 003 & -89 & 27 & 279 & -83 & 13 & -147 & 106 & 35,79 & $\mathrm{~V}^{+}$ & $\mathrm{F}^{-}$ & - & 1,86 & $-3,48$ & 0,00 \\
\hline 010 & -112 & 14 & 73 & -3 & 110 & -83 & 66 & 39,52 & $\mathrm{~V}^{+}$ & $F^{-}$ & $\mathrm{V}^{-}$ & 2,30 & $-2,47$ & 1,95 \\
\hline 011 & -100 & 4 & 136 & -25 & 81 & -97 & 74 & 37,57 & $\mathrm{~V}^{+}$ & $\mathrm{F}^{-}$ & $\mathrm{V}^{-}$ & 2,13 & $-2,04$ & 0,09 \\
\hline 012 & -104 & 44 & 183 & -58 & 53 & -117 & 93 & 37,48 & $\mathrm{~V}^{+}$ & $\mathrm{F}^{-}$ & $\mathrm{F}^{-}$ & 2,04 & $-1,54$ & $-1,79$ \\
\hline 013 & -86 & 68 & 247 & -82 & 18 & -165 & 111 & 39,27 & $\mathrm{~V}^{+}$ & $F^{-}$ & - & 1,96 & $-1,11$ & 0,00 \\
\hline 020 & -113 & 11 & 74 & 8 & 107 & -87 & 67 & 41,99 & $\mathrm{~V}^{+}$ & $\mathrm{F}^{-}$ & $\mathrm{V}^{-}$ & 2,38 & $-0,09$ & 2,38 \\
\hline 021 & -103 & -5 & 115 & -16 & 86 & -77 & 67 & 39,61 & $\mathrm{~V}^{+}$ & $\mathrm{F}^{+}$ & $\mathrm{V}^{-}$ & 2,21 & $-0,34$ & 0,59 \\
\hline 022 & -98 & 35 & 144 & -47 & 59 & -93 & 79 & 39,02 & $\mathrm{~V}^{+}$ & $\mathrm{V}^{-}$ & $\mathrm{F}^{-}$ & 2,12 & 0,77 & $-1,36$ \\
\hline 023 & -75 & 59 & 190 & -66 & 26 & -134 & 92 & 40,38 & $\mathrm{~V}^{+}$ & $\mathrm{V}^{-}$ & - & 2,03 & 1,19 & 0,00 \\
\hline 030 & -111 & -40 & 52 & 31 & 119 & -51 & 67 & 42,08 & $\mathrm{~V}^{+}$ & - & $\mathrm{V}^{-}$ & 2,54 & 0,00 & 2,81 \\
\hline 031 & -101 & -10 & 70 & 0 & 92 & -52 & 54 & 39,27 & $\mathrm{~V}^{+}$ & - & $\mathrm{V}^{-}$ & 2,29 & 0,00 & 1,02 \\
\hline 032 & -81 & -10 & 92 & -20 & 72 & -53 & 55 & 38,25 & $\mathrm{~V}^{+}$ & - & $\mathrm{F}^{-}$ & 2,29 &, 00 & $-0,94$ \\
\hline 033 & -57 & 5 & 109 & -40 & 49 & -66 & 54 & 39,19 & $\mathrm{~V}^{+}$ & - & - & 2,12 & 0,00 & 0,00 \\
\hline 100 & -28 & 33 & -28 & -8 & 123 & -92 & 52 & 36,98 & $\mathrm{~V}^{+}$ & $\mathrm{F}^{-}$ & $\mathrm{F}^{+}$ & 1,01 & $-4,84$ & $-1,71$ \\
\hline 101 & -40 & 11 & 55 & -34 & 96 & -88 & 54 & 35,27 & $\mathrm{~V}^{+}$ & $\mathrm{F}^{-}$ & $\mathrm{F}^{-}$ & 0,94 & $-4,43$ & $-0,26$ \\
\hline 102 & -45 & 6 & 131 & -57 & 61 & -97 & 66 & 35,53 & $\mathrm{~V}^{+}$ & $\mathrm{F}^{-}$ & $\mathrm{F}^{-}$ & 0,85 & $-3,99$ & $-2,12$ \\
\hline 103 & -41 & 24 & 212 & -80 & 35 & -149 & 90 & 37,65 & $\mathrm{~V}^{+}$ & $F^{*}$ & - & 0,69 & $-3,58$ & 0,00 \\
\hline 110 & -34 & 31 & 19 & -5 & 118 & -130 & 56 & 41 , & $\mathrm{V}^{+}$ & $\mathrm{F}$ & $\mathrm{V}^{-}$ & 1,19 & $-2,55$ & 2,12 \\
\hline 111 & -46 & 5 & 80 & -33 & 91 & -96 & 59 & 39 , & $\mathrm{V}^{+}$ & $\mathrm{F}^{-}$ & $\mathrm{V}^{-}$ & 1,10 & $-2,12$ & 0,18 \\
\hline 112 & -47 & 49 & 135 & -59 & 65 & -143 & 83 & 39,52 & $\mathrm{~V}^{+}$ & $F^{-}$ & $\mathrm{F}^{-}$ & 0,94 & $-1,62$ & $-1,71$ \\
\hline 113 & -33 & 21 & 204 & -73 & 33 & -153 & 86 & 41,23 & $\mathrm{~V}^{+}$ & $\mathrm{F}^{-}$ & - & 0,76 & $-1,18$ & 0,00 \\
\hline 120 & -50 & 19 & 31 & -1 & 110 & -109 & 53 & 44,37 & $\mathrm{~V}^{+}$ & $\mathrm{F}^{-}$ & $\mathrm{V}^{-}$ & 1,28 & $-0,25$ & 2,55 \\
\hline 121 & -47 & 2 & 77 & -22 & 88 & -99 & 56 & 41,82 & $\mathrm{~V}^{+}$ & $\mathrm{V}^{-}$ & $\mathrm{V}^{-}$ & 1,19 & 0,26 & 0,68 \\
\hline 122 & -48 & 41 & 112 & -48 & 60 & -117 & 71 & 41,14 & $\mathrm{~V}^{+}$ & $\mathrm{V}^{*}$ & $\mathrm{~F}^{-}$ & 1,10 & 0,60 & $-1,27$ \\
\hline 123 & -37 & 54 & 156 & -69 & 35 & -139 & 82 & 42,41 & $\mathrm{~V}^{+}$ & $\mathrm{V}^{-}$ & - & 0,94 & 1,10 & 0,00 \\
\hline 130 & -50 & -31 & 21 & 20 & 112 & -71 & 51 & 44,62 & $\mathrm{~V}^{+}$ & - & $\mathrm{V}^{-}$ & 1,37 & 0,00 & 3,06 \\
\hline 131 & -56 & -11 & 41 & -11 & 91 & -53 & 44 & 41,56 & $\mathrm{~V}^{+}$ & - & $\mathrm{V}^{-}$ & 1,28 & 0,00 & 1,02 \\
\hline 132 & -43 & -12 & 69 & -28 & 69 & -56 & 46 & 40,54 & $\mathrm{~V}^{+}$ & - & $F^{-}$ & 1,11 & 0,00 & $-0,77$ \\
\hline 133 & -27 & 2 & 93 & -45 & 46 & -70 & 47 & 41,31 & $\mathrm{~V}^{+}$ & - & - & 1,02 & 0,00 & 0,00 \\
\hline
\end{tabular}


Apêndice 14. Indices DRIS, calculados pelo procedimento proposto por Beaufils (1973), diagnose para a correção de N, P e K, resposta líquida da produtividade, diagnose para o nutriente deficiente e a resposta da produtividade, para os dados, dos tratamentos com as doses 2 e 3 de $\mathrm{N}$ em todas as combinações de doses de $\mathrm{P}$ e $\mathrm{K}$, do ensaio de adubação $\mathrm{N}, \mathrm{P}$ e $\mathrm{K}$ conduzido em Matão/SP.

\begin{tabular}{|c|c|c|c|c|c|c|c|c|c|c|c|c|c|c|}
\hline \multirow[b]{2}{*}{ Trat. } & \multicolumn{6}{|c|}{ Índices DRIS } & \multirow[b]{2}{*}{$\mathrm{BN}$} & \multirow[b]{2}{*}{ Prod. } & \multicolumn{3}{|c|}{ Diagnose } & \multicolumn{3}{|c|}{ Resposta } \\
\hline & $\overline{\mathrm{N}}$ & $P$ & $\mathrm{~K}$ & $\mathrm{Ca}$ & $\mathrm{Mg}$ & $\bar{S}$ & & & $\mathrm{~N}$ & $\mathbf{P}$ & $\mathrm{K}$ & $\bar{d}\left(Y_{N}\right)$ & $\mathrm{d}\left(\mathrm{Y}_{\mathrm{P}}\right)$ & $\bar{d}\left(Y_{K}\right)$ \\
\hline & & & & & & & & $\mathrm{tha}^{-1}$ & & & & & $\mathrm{ha}^{-1}$ & \\
\hline 200 & 22 & 35 & -74 & -16 & 125 & -91 & 61 & 37,99 & $\mathrm{~V}^{-}$ & $\mathrm{F}^{*}$ & $\mathrm{~F}^{+}$ & 0,00 & $-5,02$ & $-1,78$ \\
\hline 201 & 3 & 10 & 22 & -40 & 95 & -89 & 43 & 36,21 & $\mathrm{~V}^{-}$ & $\mathrm{F}^{-}$ & $\mathrm{F}^{-}$ & 0,09 & $-4,59$ & $-0,17$ \\
\hline 202 & -2 & 9 & 108 & -59 & & -127 & 63 & 36,38 & $\mathrm{~F}^{+}$ & $\mathrm{F}^{-}$ & $\mathrm{F}^{-}$ & $-0,26$ & $-4,08$ & $-1,96$ \\
\hline 203 & 2 & -28 & 193 & -74 & 48 & -142 & 81 & 38,34 & $\mathrm{~V}^{-}$ & $\mathrm{V}^{+}$ & - & 0,35 & 3,65 & 0,00 \\
\hline 210 & 12 & 32 & -5 & -17 & 108 & -130 & 51 & 43,01 & $\mathrm{~F}^{-}$ & $\mathrm{F}^{-}$ & $\mathrm{F}^{+}$ & $-0,08$ & $-2,64$ & $-2,21$ \\
\hline 211 & 1 & 9 & 62 & -39 & 92 & -125 & 55 & 40,80 & $\mathrm{~V}^{-}$ & $\mathrm{F}^{-}$ & $\mathrm{V}^{-}$ & 0,00 & $-2,21$ & 0,34 \\
\hline 212 & -3 & 4 & 125 & -58 & 67 & -135 & 65 & 40,46 & $\mathrm{~F}^{+}$ & $\mathrm{F}$ & $\mathrm{F}^{-}$ & $-0,17$ & $-1,78$ & $-1,53$ \\
\hline 213 & -4 & 14 & 191 & -79 & & -162 & 82 & 41,99 & $\mathrm{~F}^{+}$ & $\mathrm{F}$ & - & $-0,26$ & $-1,36$ & 0,00 \\
\hline 220 & -5 & 20 & 19 & -13 & & -109 & 42 & 45,65 & $\mathrm{~V}^{+}$ & $F$ & $\mathrm{~V}^{-}$ & 0,16 & $-0,34$ & 2,64 \\
\hline 221 & -11 & 0 & 70 & -32 & & -102 & 48 & 43,01 & $\mathrm{~V}^{+}$ & $\mathrm{V}^{-}$ & $\mathrm{V}^{-}$ & 0,08 & 0,17 & 0,77 \\
\hline 222 & -10 & -6 & 117 & -51 & 61 & -112 & 60 & 42,24 & $\mathrm{~F}^{+}$ & $\mathrm{F}^{+}$ & $\mathrm{F}^{-}$ & $-0,08$ & $-0,59$ & $-1,11$ \\
\hline 223 & -3 & 6 & 164 & -68 & 35 & -134 & 68 & 43,35 & $\mathrm{~F}^{+}$ & $\mathrm{V}^{-}$ & - & $-0,17$ & 1,02 & 0,00 \\
\hline 230 & -10 & -31 & 20 & 4 & 89 & -72 & 38 & 45,99 & $\mathrm{~V}^{+}$ & - & $\mathrm{V}^{-}$ & 0,25 & 0,00 & 3,15 \\
\hline 231 & -24 & -12 & 46 & -23 & 68 & -56 & 38 & 42,84 & $\mathrm{~V}^{+}$ & - & $\mathrm{V}^{-}$ & 0,17 & 0,00 & 1,19 \\
\hline 232 & -18 & -15 & 76 & -39 & 56 & -61 & 44 & 41,65 & $\mathrm{~F}^{+}$ & - & $F^{-}$ & 0,00 & 0,00 & $-0,68$ \\
\hline 233 & -4 & -3 & 106 & -52 & 31 & -77 & 46 & 42,33 & $\mathrm{~F}^{+}$ & - & - & $-0,09$ & 0,00 & 0,00 \\
\hline 300 & 62 & 32 & -78 & -31 & 111 & -96 & 68 & 37,99 & - & $F^{-}$ & $\mathrm{F}^{+}$ & 0,00 & $-5,10$ & $-1,87$ \\
\hline 301 & 33 & 5 & 21 & -52 & 91 & -97 & 50 & 36,12 & - & $\mathrm{F}^{-}$ & $V^{-}$ & 0,00 & $-4,68$ & 0,00 \\
\hline 302 & 29 & -49 & 111 & -62 & 69 & -98 & 70 & 36,12 & - & $\mathrm{V}^{+}$ & $\mathrm{F}^{-}$ & 0,00 & 4,17 & $-1,87$ \\
\hline 303 & 18 & -44 & 196 & -82 & & -127 & 84 & 37,99 & - & $\mathrm{V}^{+}$ & - & 0,00 & 3,74 & 0,00 \\
\hline 310 & 38 & 21 & -2 & -37 & 90 & -110 & 50 & 43,09 & - & $\mathrm{F}^{-}$ & $\mathrm{F}^{+}$ & 0,00 & $-2,72$ & $-2,29$ \\
\hline 311 & 21 & -3 & 71 & -56 & 74 & -107 & 55 & 40,80 & - & $\mathrm{V}^{+}$ & $\mathrm{V}^{-}$ & 0,00 & 2,29 & 0,51 \\
\hline 312 & 12 & -10 & 139 & -71 & & -118 & 66 & 40,29 & - & $\mathrm{V}^{+}$ & $F^{-}$ & 0,00 & 1,87 & $-1,44$ \\
\hline 313 & 20 & -48 & 217 & -81 & & -132 & 87 & 41,73 & - & $\mathrm{V}^{+}$ & - & 0,00 & 1,45 & 0,00 \\
\hline 320 & 26 & 17 & 39 & -31 & & -115 & 49 & 45,81 & - & $F^{-}$ & $\mathrm{V}^{-}$ & 0,00 & $-0,43$ & 2,72 \\
\hline 321 & 10 & -11 & 90 & -50 & 47 & -86 & 49 & 43,09 & - & $\mathrm{F}^{+}$ & $\mathrm{V}^{-}$ & 0,00 & $-0,08$ & 0,93 \\
\hline 322 & 4 & -17 & 140 & -65 & 32 & -95 & 59 & 42,16 & - & $\mathrm{F}^{+}$ & $\mathrm{F}^{-}$ & 0,00 & $-0,51$ & $-1,02$ \\
\hline 323 & 14 & -55 & 201 & -73 & 19 & -106 & 78 & 43,18 & - & $\mathrm{F}^{+}$ & - & 0,00 & $-0,94$ & 0,00 \\
\hline 330 & 13 & -40 & 51 & -18 & 50 & -55 & 38 & 46,24 & - & - & $\mathrm{V}^{-}$ & 0,00 & 0,00 & 3,23 \\
\hline 331 & 1 & -67 & 88 & -37 & 46 & -32 & 45 & 43,01 & - & - & $\mathrm{V}^{-}$ & 0,00 & 0,00 & 1,36 \\
\hline 332 & 2 & -71 & 122 & -48 & 33 & -37 & 52 & 41,65 & - & - & $\mathrm{F}^{-}$ & 0,00 & 0,00 & $-0,59$ \\
\hline 333 & 5 & -66 & 154 & -62 & 2 & -34 & 54 & 42,24 & - & - & - & 0,00 & 0,00 & 0,00 \\
\hline
\end{tabular}


Apêndice 15. Índices DRIS, calculados pelo procedimento proposto por Beaufils (1973), diagnose para a correção de $\mathrm{N}, \mathrm{P}$ e K, resposta líquida da produtividade, diagnose para o nutriente deficiente e a resposta da produtividade, para os dados, dos tratamentos com as doses 0 e $1 \mathrm{de} \mathrm{N}$ em todas as combinações de doses de $\mathrm{P}$ e $\mathrm{K}$, do ensaio de adubação $\mathrm{N}, \mathrm{P}$ e $\mathrm{K}$ conduzido em Monte Azul/SP.

\begin{tabular}{|c|c|c|c|c|c|c|c|c|c|c|c|c|c|c|}
\hline \multirow[b]{2}{*}{ Trat. } & \multicolumn{6}{|c|}{ Índices DRIS } & \multirow[b]{2}{*}{$\mathrm{BN}$} & \multirow[b]{2}{*}{ Prod. } & \multicolumn{3}{|c|}{ Diagnose } & \multicolumn{3}{|c|}{ Resposta } \\
\hline & $\overline{\mathrm{N}}$ & $\mathrm{P}$ & $\mathrm{K}$ & $\mathrm{Ca}$ & $\mathrm{Mg}$ & $\bar{S}$ & & & $\mathrm{~N}$ & $\mathrm{P}$ & $\mathrm{K}$ & $d\left(Y_{N}\right)$ & $d\left(Y_{P}\right)$ & $\mathrm{d}\left(\mathrm{Y}_{\mathrm{K}}\right)$ \\
\hline & & & & & & & & $\mathrm{tha}^{-1}$ & & & & & tha & \\
\hline 000 & -64 & 177 & 70 & & -139 & -85 & 96 & 20,00 & $\mathrm{~V}^{+}$ & $\mathrm{V}^{-}$ & $\mathrm{V}^{-}$ & 6,53 & 0,42 & 1,25 \\
\hline 001 & -61 & 160 & 3 & 77 & -134 & -45 & 80 & 18,75 & $\mathrm{~V}^{+}$ & $\mathrm{V}^{-}$ & $\mathrm{V}^{-}$ & 6,27 & 0,17 & 0,17 \\
\hline 002 & -65 & 123 & -24 & 94 & -102 & -25 & 72 & 18,58 & $\mathrm{~V}^{+}$ & $\mathrm{F}^{-}$ & $\mathrm{V}^{+}$ & 6,11 & $-0,17$ & 0,92 \\
\hline 003 & -85 & 112 & -5 & 85 & -73 & -34 & 66 & 19,50 & $\mathrm{~V}^{+}$ & $\mathrm{F}^{-}$ & - & 5,86 & $-0,42$ & 0,00 \\
\hline 010 & -87 & 167 & 43 & 40 & -102 & -60 & 83 & 19,58 & $\mathrm{~V}^{+}$ & $\mathrm{F}^{-}$ & $\mathrm{V}^{-}$ & 6,20 & $-0,26$ & 1,00 \\
\hline 011 & -84 & 201 & -11 & 76 & -121 & -60 & 92 & 18,58 & $\mathrm{~V}^{+}$ & $\mathrm{F}^{-}$ & $\mathrm{V}^{+}$ & 5,94 & $-0,59$ & 0,17 \\
\hline 012 & -87 & 163 & -19 & 96 & -112 & -41 & 86 & 18,75 & $\mathrm{~V}^{+}$ & $\mathrm{F}^{-}$ & $\mathrm{V}^{+}$ & 5,69 & $-0,83$ & 1,17 \\
\hline 013 & -98 & 105 & 21 & 92 & -79 & -42 & 73 & 19,92 & $\mathrm{~V}^{+}$ & $\mathrm{F}^{-}$ & - & 5,52 & $-1,17$ & 0,00 \\
\hline 020 & -129 & 194 & 32 & 18 & -63 & -52 & 81 & 19,84 & $\mathrm{~V}^{+}$ & $\mathrm{F}^{-}$ & $\mathrm{V}^{-}$ & 5,85 & $-1,00$ & 0,67 \\
\hline 021 & -116 & 185 & 2 & 61 & -90 & -41 & 83 & 19,17 & $\mathrm{~V}^{+}$ & $F^{-}$ & $\mathrm{F}^{-}$ & 5,52 & $-1,25$ & $-0,41$ \\
\hline 022 & -121 & 144 & 9 & 79 & -84 & -27 & 77 & 19,58 & $\mathrm{~V}^{+}$ & $\mathrm{F}$ & $\mathrm{F}^{-}$ & 5,28 & $-1,51$ & $-1,51$ \\
\hline 023 & -140 & 130 & 58 & 73 & -78 & -42 & 87 & 21,09 & $\mathrm{~V}^{+}$ & $\mathrm{F}^{-}$ & - & 5,02 & $-1,76$ & 0,00 \\
\hline 030 & -172 & 182 & 56 & -6 & -26 & -33 & 79 & 20,84 & $\mathrm{~V}^{+}$ & - & $\mathrm{V}^{-}$ & 5,44 & 0,00 & 0,42 \\
\hline 031 & -173 & 206 & 38 & 25 & -56 & -40 & 90 & 20,42 & $\mathrm{~V}^{+}$ & - & $\mathrm{F}^{-}$ & 5,19 & 0,00 & $-0,67$ \\
\hline 032 & -170 & 174 & 66 & 48 & -65 & -52 & 96 & 21,09 & $\mathrm{~V}^{+}$ & - & $\mathrm{F}^{*}$ & 4,94 & 1,00 & $-1,76$ \\
\hline 033 & -190 & 158 & 120 & 44 & -62 & -70 & 107 & 22,85 & $\mathrm{~V}^{+}$ & - & - & 4,68 & 0,00 & 0,00 \\
\hline 100 & -14 & 109 & 71 & 62 & -120 & -109 & 81 & 26,53 & $\mathrm{~V}^{+}$ & $\mathrm{V}^{-}$ & $\mathrm{V}^{-}$ & 3,93 & 0,75 & 1,51 \\
\hline 101 & -7 & 138 & 15 & 87 & -121 & -113 & 80 & 25,02 & $\mathrm{~V}^{+}$ & $\mathrm{V}^{-}$ & $\mathrm{V}^{-}$ & 3,69 & 0,50 & 0,33 \\
\hline 102 & -19 & 141 & -6 & 88 & -97 & -108 & 77 & 24,69 & $\mathrm{~V}^{+}$ & $\mathrm{V}^{-}$ & $\mathrm{V}^{+}$ & 3,43 & 0,25 & 0,67 \\
\hline 103 & -25 & 84 & 31 & 78 & -63 & -105 & 64 & 25,36 & $\mathrm{~V}^{+}$ & $\mathrm{F}^{-}$ & - & 3,18 & $-0,08$ & 0,00 \\
\hline 110 & -22 & 109 & 28 & 71 & -80 & -105 & 69 & 25,78 & $\mathrm{~V}^{+}$ & $\mathrm{V}^{-}$ & $\mathrm{V}^{-}$ & 3,51 & 0,09 & 1,26 \\
\hline 111 & -23 & 130 & -18 & 96 & -103 & -83 & 76 & 24,52 & $\mathrm{~V}^{+}$ & $\mathrm{F}^{-}$ & $\mathrm{F}^{+}$ & 3,27 & $-0,17$ & $-0,08$ \\
\hline 112 & -24 & 141 & -16 & 105 & -98 & -108 & 82 & 24,44 & $\mathrm{~V}^{+}$ & $\mathrm{F}^{-}$ & $\mathrm{V}^{+}$ & 3,01 & $-0,42$ & 1,00 \\
\hline 113 & -48 & 114 & 29 & 80 & -77 & -99 & 75 & 25,44 & $\mathrm{~V}^{+}$ & $F^{-}$ & - & 2,76 & $-0,67$ & 0,00 \\
\hline 120 & -48 & 100 & 10 & 65 & -50 & -77 & 58 & 25,69 & $\mathrm{~V}^{+}$ & $\mathrm{F}^{-}$ & $\mathrm{V}^{-}$ & 3,10 & $-0,59$ & 1,00 \\
\hline 121 & -42 & 125 & -18 & 91 & -69 & -87 & 72 & 24,69 & $\mathrm{~V}^{+}$ & $\mathrm{F}^{-}$ & $\mathrm{V}^{+}$ & 2,93 & $-0,92$ & 0,17 \\
\hline 122 & -47 & 126 & -2 & 96 & -86 & -87 & 74 & 24,86 & $\mathrm{~V}^{+}$ & $\mathrm{F}^{-}$ & $\mathrm{V}^{+}$ & 2,67 & $-1,17$ & 1,25 \\
\hline 123 & -62 & 109 & 59 & 82 & -82 & -106 & 83 & 26,11 & $\mathrm{~V}^{+}$ & $\mathrm{F}^{-}$ & - & 2,43 & $-1,42$ & 0,00 \\
\hline 130 & -85 & 137 & 16 & 42 & -16 & -95 & 65 & 26,28 & $\mathrm{~V}^{+}$ & - & $\mathrm{V}^{-}$ & 2,76 & 0,00 & 0,67 \\
\hline 131 & -85 & 152 & -2 & 64 & -49 & -81 & 72 & 25,61 & $\mathrm{~V}^{+}$ & - & $\mathrm{V}^{+}$ & 2,51 & 0,00 & 0,42 \\
\hline 132 & -88 & 158 & 36 & 71 & -65 & -112 & 88 & 26,03 & $\mathrm{~V}^{+}$ & - & $\mathrm{F}^{*}$ & 2,34 & 0,00 & $-1,50$ \\
\hline 133 & -100 & 143 & 110 & 60 & -79 & -133 & 104 & 27,53 & $\mathrm{~V}^{+}$ & - & - & 2,10 & 0,00 & 0,00 \\
\hline
\end{tabular}


Apêndice 16. Índices DRIS, calculados pelo procedimento proposto por Beaufils (1973), diagnose para a correção de $\mathrm{N}, \mathrm{P}$ e K, resposta líquida da produtividade, diagnose para o nutriente deficiente e a resposta da produtividade, para os dados, dos tratamentos com as doses 2 e 3 de $\mathrm{N}$ em todas as combinações de doses de $\mathrm{P}$ e $\mathrm{K}$, do ensaio de adubação $\mathrm{N}, \mathrm{P}$ e $\mathrm{K}$ conduzido em Monte Azul/SP.

\begin{tabular}{|c|c|c|c|c|c|c|c|c|c|c|c|c|c|c|}
\hline \multirow[b]{2}{*}{ Trat. } & \multicolumn{6}{|c|}{ Índices DRIS } & \multirow[b]{2}{*}{$\mathrm{BN}$} & \multirow[b]{2}{*}{ Prod. } & \multicolumn{3}{|c|}{ Diagnose } & \multicolumn{3}{|c|}{ Resposta } \\
\hline & $\overline{\mathrm{N}}$ & $\mathrm{P}$ & $\mathrm{K}$ & $\mathrm{Ca}$ & $\mathrm{Mg}$ & $\mathbf{S}$ & & & $\mathbf{N}$ & $P$ & $\mathrm{~K}$ & $d\left(Y_{N}\right)$ & $d\left(Y_{P}\right)$ & $d\left(Y_{K}\right)$ \\
\hline & & & & & & & & $\mathrm{tha}^{-1}$ & & & & & $\mathrm{ha}^{-1}$ & \\
\hline 200 & -1 & 72 & 68 & 77 & -93 & -123 & 72 & 30,46 & $\mathrm{~V}^{+}$ & $\mathrm{V}^{-}$ & $\mathrm{V}^{-}$ & 1,26 & 1,17 & 1,75 \\
\hline 201 & 1 & 89 & 18 & 90 & -97 & -101 & 66 & 28,71 & $\mathrm{~F}^{-}$ & $\mathrm{V}^{-}$ & $\mathrm{V}^{-}$ & $-1,00$ & 0,92 & 0,59 \\
\hline 202 & -5 & 90 & 9 & 82 & -76 & -99 & 60 & 28,12 & $\mathrm{~V}^{+}$ & $\mathrm{V}^{*}$ & $\mathrm{~F}^{-}$ & 0,75 & 0,67 & $-0,42$ \\
\hline 203 & -17 & 76 & 53 & 57 & -53 & -115 & 62 & 28,54 & $\mathrm{~V}^{+}$ & $\mathrm{V}^{-}$ & - & 0,58 & 0,34 & 0,00 \\
\hline 210 & 8 & 33 & 15 & 106 & -63 & -98 & 54 & 29,29 & $\mathrm{~F}^{-}$ & $\mathrm{V}^{-}$ & $\mathrm{V}^{-}$ & $-0,84$ & 0,50 & 1,50 \\
\hline 211 & 1 & 93 & -32 & 111 & -76 & -96 & 68 & 27,79 & $\mathrm{~F}^{-}$ & $\mathrm{V}^{-}$ & $\mathrm{F}^{+}$ & $-0,67$ & 0,17 & $-0,34$ \\
\hline 212 & -4 & 91 & -18 & 104 & -76 & -97 & 65 & 27,45 & $\mathrm{~V}^{+}$ & $\mathrm{F}^{-}$ & $\mathrm{V}^{+}$ & 0,42 & $-0,08$ & 0,75 \\
\hline 213 & -18 & 73 & 43 & 77 & -56 & 119 & 64 & 28,20 & $\mathrm{~V}^{+}$ & $F^{-}$ & - & 0,17 & $-0,34$ & 0,00 \\
\hline 220 & -20 & 71 & -29 & 98 & -39 & -82 & 57 & 28,79 & $\mathrm{~V}^{+}$ & $F^{-}$ & $\mathrm{F}^{+}$ & 0,50 & $-0,25$ & $-1,17$ \\
\hline 221 & -12 & 93 & -47 & 118 & -58 & -94 & 70 & 27,62 & $\mathrm{~V}^{+}$ & $\mathrm{F}^{-}$ & $\mathrm{F}^{+}$ & 0,25 & $-0,50$ & $-0,09$ \\
\hline 222 & -17 & 89 & -20 & 109 & -61 & -99 & 66 & 27,53 & $\mathrm{~F}^{+}$ & $F^{-}$ & $\mathrm{V}^{+}$ & 0,00 & $-0,84$ & 1,01 \\
\hline 223 & -26 & 69 & 57 & 83 & -60 & -124 & 70 & 28,54 & $\mathrm{~F}^{+}$ & $F^{-}$ & - & $-0,17$ & $-1,09$ & 0,00 \\
\hline 230 & -43 & 69 & -35 & 89 & 2 & -82 & 53 & 29,04 & $\mathrm{~V}^{+}$ & - & $\mathrm{F}^{+}$ & 0,08 & 0,00 & $-0,92$ \\
\hline 231 & -39 & 80 & -40 & 102 & -32 & -71 & 61 & 28,12 & $\mathrm{~F}^{+}$ & - & $\mathrm{V}^{+}$ & $-0,08$ & 0,00 & 0,25 \\
\hline 232 & -37 & 83 & 8 & 100 & $-49-$ & 105 & 64 & 28,37 & $\mathrm{~F}^{+}$ & - & $\mathrm{F}^{-}$ & $-0,42$ & 0,00 & $-1,26$ \\
\hline 233 & -63 & 99 & 86 & 67 & $-73-$ & -118 & 84 & 29,63 & $\mathrm{~F}^{+}$ & - & - & $-0,59$ & 0,00 & 0,00 \\
\hline 300 & -23 & -39 & 58 & 90 & -40 & -45 & 49 & 31,72 & - & $\mathrm{F}^{+}$ & $\mathrm{V}^{-}$ & 0,00 & $-1,59$ & 2,01 \\
\hline 301 & -22 & 29 & 17 & 89 & -46 & -66 & 45 & 29,71 & - & $\mathrm{V}^{-}$ & $\mathrm{V}^{-}$ & 0,00 & 1,25 & 0,84 \\
\hline 302 & -31 & 74 & 20 & 68 & -51 & -80 & 54 & 28,8 & - & $\mathrm{V}^{-}$ & $\mathrm{F}^{-}$ & 0,00 & 1,00 & $-0,25$ \\
\hline 303 & -48 & 48 & 68 & 30 & -23 & -76 & 49 & 29,1 & - & $\mathrm{V}^{-}$ & - & 0,00 & 0,75 & 0,00 \\
\hline 310 & -15 & -29 & -16 & 123 & -32 & -32 & 41 & 30,13 & - & $\mathrm{F}^{+}$ & $\mathrm{F}^{+}$ & 0,00 & $-0,84$ & $-1,67$ \\
\hline 311 & -13 & 36 & -47 & 122 & -42 & -57 & 53 & 28,46 & - & $\mathrm{V}^{-}$ & $\mathrm{F}^{+}$ & 0,00 & 0,59 & $-0,59$ \\
\hline 312 & -14 & 33 & -20 & 105 & -43 & -61 & 46 & 27,87 & - & $\mathrm{V}^{-}$ & $\mathrm{V}^{+}$ & 0,00 & 0,34 & 0,50 \\
\hline 313 & -40 & 50 & 42 & 58 & -36 & -73 & 50 & 28,37 & - & $\mathrm{V}^{-}$ & - & 0,00 & 0,00 & 0,00 \\
\hline 320 & -21 & -23 & -71 & 139 & -1 & -23 & 46 & 29,29 & - & $\mathrm{F}^{+}$ & $\mathrm{F}^{+}$ & 0,00 & $-0,17$ & $-1,42$ \\
\hline 321 & -14 & 43 & -85 & 142 & -38 & -48 & 62 & 27,87 & - & $\mathrm{F}^{-}$ & $\mathrm{F}^{+}$ & 0,00 & $-0,17$ & $-0,34$ \\
\hline 322 & -18 & 35 & -38 & 122 & -43 & -58 & 52 & 27,53 & - & $F^{*}$ & $\mathrm{~V}^{+}$ & 0,00 & $-0,42$ & 0,84 \\
\hline 323 & -42 & 51 & 40 & 74 & -51 & -72 & 55 & 28,37 & - & $\mathrm{F}^{*}$ & - & 0,00 & $-0,67$ & 0,00 \\
\hline 330 & -31 & $-19-$ & 103 & 141 & 29 & -17 & 57 & 29,12 & - & - & $\mathrm{F}^{+}$ & 0,00 & 0,00 & $-1,08$ \\
\hline 331 & -36 & 34 & -98 & 135 & -12 & -24 & 57 & 28,04 & - & - & $\mathrm{F}^{+}$ & 0,00 & 0,00 & $-0,09$ \\
\hline 332 & -32 & 31 & -26 & 119 & -30 & -62 & 50 & 27,95 & - & - & $\mathrm{V}^{+}$ & 0,00 & 0,00 & 1,09 \\
\hline 333 & -57 & 45 & 62 & 71 & -41 & -80 & 59 & 29,04 & - & - & - & 0,00 & 0,00 & 0,00 \\
\hline
\end{tabular}


Apêndice 17. Índices DRIS, calculados pelo procedimento proposto por Beaufils (1973), diagnose para a correção de N, P e K, resposta líquida da produtividade, diagnose para o nutriente deficiente e a resposta da produtividade, para os dados, dos tratamentos com as doses 0 e $1 \mathrm{de} \mathrm{N}$ em todas as combinações de doses de $\mathrm{P}$ e $\mathrm{K}$, do ensaio de adubação $\mathrm{N}, \mathrm{P}$ e K conduzido em Olímpia/SP.

\begin{tabular}{|c|c|c|c|c|c|c|c|c|c|c|c|c|c|}
\hline \multirow[b]{2}{*}{ Trat. } & \multicolumn{5}{|c|}{ Índices DRIS } & \multirow[b]{2}{*}{$\mathrm{BN}$} & \multirow[b]{2}{*}{ Prod. } & \multicolumn{3}{|c|}{ Diagnose } & \multicolumn{3}{|c|}{ Resposta } \\
\hline & $\mathrm{N}$ & $P$ & $\mathrm{~K} \mathrm{Ca}$ & $\mathrm{Mg}$ & $\bar{S}$ & & & $\mathrm{~N}$ & $P$ & $\mathrm{~K}$ & $\mathrm{~d}\left(\mathrm{Y}_{\mathrm{N}}\right)$ & $\mathrm{d}\left(\mathrm{Y}_{\mathrm{P}}\right)$ & $d\left(Y_{K}\right)$ \\
\hline & & & & & & & $\mathrm{tha}^{-1}$ & & & & & $t$ ha' & \\
\hline 000 & 74 & 80 & $-22-174$ & 88 & -46 & 81 & 26,86 & $\mathrm{~V}^{-}$ & $\mathrm{V}^{-}$ & $\mathrm{F}^{+}$ & 0,25 & 0,77 & $-0,77$ \\
\hline 001 & 41 & 56 & $-57-136$ & 106 & -12 & 68 & 26,09 & $\mathrm{~V}^{-}$ & $\mathrm{V}^{-}$ & $\mathrm{F}^{+}$ & 0,08 & 0,51 & $-0,59$ \\
\hline 002 & 9 & 43 & $-72-130$ & 122 & 28 & 67 & 25,50 & $\mathrm{~V}^{-}$ & $\mathrm{V}^{-}$ & $\mathrm{F}^{+}$ & 0,00 & 0,25 & $-0,51$ \\
\hline 003 & -11 & 49 & $-67-146$ & 140 & 35 & 75 & 24,99 & $\mathrm{~V}^{+}$ & $\mathrm{V}^{-}$ & - & 0,17 & 0,00 & 0,00 \\
\hline 010 & 40 & 78 & $-39-162$ & 100 & -17 & 73 & 26,09 & $\mathrm{~V}^{-}$ & $\mathrm{F}^{-}$ & $\mathrm{F}^{+}$ & 0,84 & $-0,43$ & $-0,51$ \\
\hline 011 & 5 & 46 & $-69-139$ & 126 & 31 & 69 & 25,58 & $\mathrm{~V}^{-}$ & $\mathrm{F}^{-}$ & $\mathrm{F}^{+}$ & 0,67 & $-0,77$ & $-0,33$ \\
\hline 012 & -14 & 37 & $-72-139$ & 142 & 46 & 75 & 25,25 & $\mathrm{~F}^{+}$ & $\mathrm{F}^{-}$ & $\mathrm{F}^{+}$ & $-0,52$ & $-1,02$ & $-0,26$ \\
\hline 013 & -26 & 41 & $-50-162$ & 148 & 50 & 80 & 24,99 & $\mathrm{~F}^{+}$ & $\mathrm{F}^{-}$ & - & $-0,26$ & $-1,36$ & 0,00 \\
\hline 020 & 27 & 46 & $-42-154$ & 114 & 7 & 65 & & $\mathrm{~V}^{-}$ & $\mathrm{F}$ & $\mathrm{F}^{+}$ & 1,36 & $-1,79$ & $-0,17$ \\
\hline 021 & 0 & 61 & $-66-140$ & 125 & 21 & 69 & 26,35 & $\mathrm{~V}^{-}$ & $\mathrm{F}^{*}$ & $\mathrm{~F}^{+}$ & 1,19 & $-2,04$ & $-0,08$ \\
\hline 022 & -20 & 44 & $-62-152$ & 137 & 53 & 78 & 26,27 & $\mathrm{~F}^{+}$ & $\mathrm{F}^{-}$ & $\mathrm{V}^{+}$ & $-1,02$ & $-2,37$ & 0,08 \\
\hline 023 & -16 & 52 & $-24-180$ & 133 & 35 & 73 & 26,35 & $\mathrm{~F}^{+}$ & $F^{-}$ & - & $-0,85$ & $-2,63$ & 0,00 \\
\hline 030 & 27 & 72 & $-49-156$ & 97 & 8 & 68 & 28,31 & $\mathrm{~V}^{-}$ & - & $\mathrm{V}^{+}$ & 1,88 & 0,00 & 0,08 \\
\hline 031 & 15 & 41 & $-55-143$ & 112 & 30 & 66 & 28,39 & $\mathrm{~V}^{*}$ & - & $\mathrm{V}^{+}$ & 1,70 & 0,00 & 0,25 \\
\hline 032 & 9 & 27 & $-34-156$ & 114 & 39 & 63 & 28,64 & $\mathrm{~V}^{*}$ & - & $\mathrm{V}^{+}$ & 1,53 & 0,00 & 0,34 \\
\hline 033 & 18 & 34 & $9-199$ & 120 & 18 & 66 & 28,98 & $\mathrm{~V}^{-}$ & - & - & 1,36 & 0,00 & 0,00 \\
\hline 100 & 55 & 55 & $-16-155$ & 104 & -43 & 71 & 26,61 & $\mathrm{~F}^{-}$ & $\mathrm{V}^{-}$ & $\mathrm{F}^{+}$ & $-0,76$ & 1,36 & $-0,60$ \\
\hline 101 & 33 & 36 & $-51-125$ & 113 & -7 & 61 & 26,01 & $F^{*}$ & $\mathrm{~V}^{*}$ & $\mathrm{~F}^{+}$ & $-1,02$ & 1,10 & $-0,51$ \\
\hline 102 & 18 & 31 & $-63-122$ & 122 & 14 & 62 & 25,50 & $F^{-}$ & $\mathrm{V}^{-}$ & $\mathrm{F}^{+}$ & $-1,19$ & 0,77 & $-0,34$ \\
\hline 103 & 11 & 41 & $-55-141$ & 120 & 24 & 65 & 25,16 & $F^{-}$ & $\mathrm{V}^{-}$ & - & $-1,36$ & 0,43 & 0,00 \\
\hline 110 & 19 & 46 & $-47-146$ & 123 & 4 & 64 & 25,25 & $\mathrm{~F}^{-}$ & $\mathrm{V}^{-}$ & $\mathrm{F}^{+}$ & $-0,25$ & 0,09 & $-0,34$ \\
\hline 111 & 8 & 32 & $-68-122$ & 134 & 15 & 63 & 24,91 & $\mathrm{~F}^{-}$ & $\mathrm{F}^{-}$ & $\mathrm{F}^{+}$ & $-0,51$ & $-0,25$ & $-0,18$ \\
\hline 112 & 0 & 26 & $-67-124$ & 131 & 34 & 64 & 24,73 & $\mathrm{~F}^{-}$ & $F^{-}$ & $\mathrm{F}^{+}$ & $-0,69$ & $-0,52$ & 0,00 \\
\hline 113 & 5 & 38 & $-45-147$ & 129 & 20 & 64 & 24,73 & $\mathrm{~F}^{*}$ & $\mathrm{~F}^{-}$ & - & $-0,77$ & $-0,77$ & 0,00 \\
\hline 120 & 16 & 21 & $-59-126$ & 141 & 8 & 62 & 25,16 & $\mathrm{~V}^{-}$ & $\mathrm{F}^{-}$ & $\mathrm{F}^{+}$ & 0,25 & $-1,27$ & 0,00 \\
\hline 121 & 1 & 41 & $-79-120$ & 131 & 26 & 66 & 25,16 & $\mathrm{~V}^{*}$ & $F^{-}$ & $\mathrm{V}^{+}$ & 0,08 & $-1,53$ & 0,09 \\
\hline 122 & 4 & 35 & $-65-131$ & 138 & 19 & 65 & 25,25 & $\mathrm{~F}^{*}$ & $F^{*}$ & $\mathrm{~V}^{+}$ & $-0,17$ & $-1,86$ & 0,25 \\
\hline 123 & 18 & 47 & $-33-162$ & 125 & 4 & 65 & 25,50 & $\mathrm{~F}^{-}$ & $F^{-}$ & - & $-0,34$ & $-2,12$ & 0,00 \\
\hline 130 & 22 & 44 & $-73-124$ & 124 & 7 & 66 & 26,43 & $\mathrm{~V}^{-}$ & - & $\mathrm{V}^{+}$ & 0,76 & 0,00 & 0,26 \\
\hline 131 & 23 & 24 & $-76-116$ & 131 & 12 & 64 & 26,69 & $\mathrm{~V}^{-}$ & - & $\mathrm{V}^{+}$ & 0,60 & 0,00 & 0,42 \\
\hline 132 & 34 & 18 & $-52-132$ & 127 & 4 & 61 & 27,11 & $\mathrm{~V}^{-}$ & - & $\mathrm{V}^{+}$ & 0,42 & 0,00 & 0,51 \\
\hline 133 & 55 & 28 & $-5-173$ & 111 & -16 & 65 & 27,62 & $\mathrm{~V}^{-}$ & - & - & 0,17 & 0,00 & 0,00 \\
\hline
\end{tabular}


Apêndice 18. Índices DRIS, calculados pelo procedimento proposto por Beaufils (1973), diagnose para a correção de N, P e K, resposta líquida da produtividade, diagnose para o nutriente deficiente e a resposta da produtividade, para os dados, dos tratamentos com as doses 2 e 3 de $\mathrm{N}$ em todas as combinações de doses de $\mathrm{P}$ e $\mathrm{K}$, do ensaio de adubação $\mathrm{N}, \mathrm{P}$ e $\mathrm{K}$ conduzido em Olimpia/SP.

\begin{tabular}{|c|c|c|c|c|c|c|c|c|c|c|c|c|c|}
\hline \multirow[b]{2}{*}{ Trat. } & \multicolumn{5}{|c|}{ Índices DRIS } & \multirow[b]{2}{*}{$\mathrm{BN}$} & \multirow[b]{2}{*}{ Prod. } & \multicolumn{3}{|c|}{ Diagnose } & \multicolumn{3}{|c|}{ Resposta } \\
\hline & $\mathrm{N}$ & $\mathrm{P}$ & $\mathrm{K} \quad \mathrm{Ca}$ & $\mathrm{Mg}$ & $\bar{S}$ & & & $\mathrm{~N}$ & $\mathrm{P}$ & $\mathbf{K}$ & $d\left(Y_{N}\right)$ & $\mathrm{d}\left(\mathrm{Y}_{\mathrm{P}}\right)$ & $\mathrm{d}\left(\mathrm{Y}_{\mathrm{K}}\right)$ \\
\hline & & & & & & & $\mathrm{tha}^{-1}$ & & & & & tha & \\
\hline 200 & 40 & 46 & $-31-153$ & 121 & -24 & 69 & 27,37 & $\mathrm{~F}^{-}$ & $\mathrm{V}^{-}$ & $\mathrm{F}^{+}$ & $-1,96$ & 1,87 & $-0,34$ \\
\hline 201 & 31 & 32 & $-69-130$ & 123 & 14 & 67 & 27,03 & $\mathrm{~F}^{-}$ & $\mathrm{V}^{-}$ & $\mathrm{F}^{+}$ & $-2,13$ & 1,61 & $-0,34$ \\
\hline 202 & 32 & 37 & $-78-126$ & 116 & 20 & 68 & 26,69 & $\mathrm{~F}^{-}$ & $\mathrm{V}^{-}$ & $\mathrm{F}^{+}$ & $-2,29$ & 1,27 & $-0,17$ \\
\hline 203 & 40 & 54 & $-64-149$ & 107 & 12 & 71 & 26,52 & $\mathrm{~F}^{-}$ & $\mathrm{V}^{*}$ & - & $-2,55$ & 1,02 & 0,00 \\
\hline 210 & 16 & 44 & $-68-137$ & 144 & 2 & 69 & 25,50 & $\mathrm{~F}^{-}$ & $\mathrm{V}^{-}$ & $\mathrm{F}^{+}$ & $-1,44$ & 0,59 & $-0,08$ \\
\hline 211 & 14 & 33 & $-92-118$ & 146 & 17 & 70 & 25,42 & $\mathrm{~F}^{-}$ & $\mathrm{V}^{-}$ & $\mathrm{F}^{+}$ & $-1,61$ & 0,34 & 0,00 \\
\hline 212 & 24 & 35 & $-91-123$ & 137 & 18 & 71 & 25,42 & $\mathrm{~F}^{-}$ & $\mathrm{V}^{-}$ & $\mathrm{V}^{+}$ & $-1,78$ & 0,00 & 0,08 \\
\hline 213 & 48 & 9 & $-56-142$ & 121 & 19 & 66 & 25,50 & $F^{-}$ & $\mathrm{F}^{-}$ & - & $-1,95$ & $-0,34$ & 0,00 \\
\hline 220 & 13 & 13 & $-97-115$ & 159 & 27 & 71 & 24,91 & $\mathrm{~F}^{-}$ & $\mathrm{F}^{-}$ & $\mathrm{V}^{+}$ & $-0,93$ & $-0,76$ & 0,17 \\
\hline 221 & 23 & $0-$ & $-103-106$ & 148 & 38 & 70 & 25,08 & $\mathrm{~F}^{-}$ & $\mathrm{F}^{-}$ & $\mathrm{V}^{+}$ & $-1,10$ & $-1,01$ & 0,34 \\
\hline 222 & 36 & 0 & $-93-116$ & 136 & 37 & 70 & 25,42 & $\mathrm{~F}^{-}$ & $F^{-}$ & $\mathrm{V}^{+}$ & $-1,19$ & $-1,27$ & 0,42 \\
\hline 223 & 62 & 16 & $-50-154$ & 126 & 1 & 68 & 25,84 & $\mathrm{~F}^{-}$ & $F^{-}$ & - & $-1,45$ & $-1,61$ & 0,00 \\
\hline 230 & 26 & $41-$ & $-123-107$ & 157 & 5 & 77 & 25,67 & $\mathrm{~F}^{-}$ & - & $\mathrm{V}^{+}$ & $-0,34$ & 0,00 & 0,42 \\
\hline 231 & 41 & $25-$ & $-120-103$ & 143 & 14 & 74 & 26,09 & $\mathrm{~F}^{-}$ & - & $\mathrm{V}^{+}$ & $-0,60$ & 0,00 & 0,60 \\
\hline 232 & 68 & 27 & $-92-122$ & 132 & -13 & 76 & 26,69 & $\mathrm{~F}^{*}$ & - & $\mathrm{V}^{+}$ & $-0,76$ & 0,00 & 0,76 \\
\hline 233 & 105 & 45 & $-38-165$ & 110 & -56 & 87 & 27,45 & $\mathrm{~F}^{-}$ & - & - & $-0,86$ & 0,00 & 0,00 \\
\hline 300 & 44 & 14 & $-62-158$ & 138 & 25 & 74 & 29,33 & - & $\mathrm{V}^{-}$ & $\mathrm{F}^{+}$ & 0,00 & 2,39 & $-0,17$ \\
\hline 301 & 49 & 8 & $-98-136$ & 133 & 45 & 78 & 29,16 & - & $\mathrm{V}^{-}$ & $\mathrm{F}^{+}$ & 0,00 & 2,13 & $-0,18$ \\
\hline 302 & 68 & $21-$ & $-107-135$ & 119 & 34 & 81 & 28,98 & - & $\mathrm{V}^{-}$ & $\mathrm{V}^{+}$ & 0,00 & 1,78 & 0,09 \\
\hline 303 & 94 & 48 & $-90-162$ & 103 & 8 & 84 & 29,07 & - & $\mathrm{V}^{-}$ & - & 0,00 & 1,62 & 0,00 \\
\hline 310 & 20 & $11-$ & $-117-137$ & 172 & 51 & 85 & 26,94 & - & $\mathrm{V}^{-}$ & $\mathrm{V}^{+}$ & 0,00 & 1,10 & 0,09 \\
\hline 311 & 40 & & $-137-118$ & 157 & 49 & 85 & 27,03 & - & $\mathrm{V}^{*}$ & $\mathrm{~V}^{+}$ & 0,00 & 0,85 & 0,17 \\
\hline 312 & 62 & $19-$ & $-128-126$ & 141 & 33 & 85 & 27,20 & - & $\mathrm{V}^{-}$ & $\mathrm{V}^{+}$ & 0,00 & 0,59 & 0,25 \\
\hline 313 & 97 & $44-$ & $-100-159$ & 113 & 4 & 86 & 27,45 & - & $\mathrm{V}^{-}$ & - & 0,00 & 0,16 & 0,00 \\
\hline 320 & 23 & $27-$ & $-165-116$ & 186 & 46 & 94 & 25,84 & - & $\mathrm{F}^{-}$ & $\mathrm{V}^{+}$ & 0,00 & $-0,17$ & 0,34 \\
\hline 321 & 47 & $22-$ & $-170-107$ & 167 & 39 & 92 & 26,18 & - & $F^{-}$ & $\mathrm{V}^{+}$ & 0,00 & $-0,51$ & 0,43 \\
\hline 322 & 77 & $29-$ & $-150-123$ & 149 & 19 & 91 & 26,61 & - & $\mathrm{F}^{-}$ & $\mathrm{V}^{+}$ & 0,00 & $-0,84$ & 0,68 \\
\hline 323 & 127 & 6 & $-98-155$ & 124 & -4 & 86 & 27,29 & - & $\mathrm{F}^{-}$ & - & 0,00 & $-1,02$ & 0,00 \\
\hline 330 & 54 & $14-$ & $-214 \quad-90$ & 194 & 42 & 101 & 26,01 & - & - & $\mathrm{V}^{+}$ & 0,00 & 0,00 & 0,68 \\
\hline 331 & 85 & $5-2$ & $-201-90$ & 172 & 29 & 97 & 26,69 & - & - & $\mathrm{V}^{+}$ & 0,00 & 0,00 & 0,76 \\
\hline 332 & 124 & $9-$ & $-159-113$ & 137 & 2 & 91 & 27,45 & - & - & $\mathrm{V}^{+}$ & 0,00 & 0,00 & 0,86 \\
\hline 333 & 181 & 37 & $-92-163$ & 105 & -69 & 108 & 28,31 & - & - & - & 0,00 & 0,00 & 0,00 \\
\hline
\end{tabular}


Apêndice 19. Índices DRIS, calculados pelo procedimento proposto por Beaufils (1973), diagnose para a correção de N, P e K, resposta líquida da produtividade, diagnose para o nutriente deficiente e a resposta da produtividade, para os dados, dos tratamentos com as doses 0 e $1 \mathrm{de} \mathrm{N}$ em todas as combinações de doses de $\mathrm{P}$ e $\mathrm{K}$, do ensaio de adubação $\mathrm{N}, \mathrm{P}$ e $\mathrm{K}$ conduzido em Pirassununga/SP.

\begin{tabular}{|c|c|c|c|c|c|c|c|c|c|c|c|c|c|c|}
\hline \multirow[b]{2}{*}{ Trat. } & \multicolumn{6}{|c|}{ Índices DRIS } & \multirow[b]{2}{*}{$\mathrm{BN}$} & \multirow[b]{2}{*}{ Prod. } & \multicolumn{3}{|c|}{ Diagnose } & \multicolumn{3}{|c|}{ Resposta } \\
\hline & $\overline{\mathrm{N}}$ & $\bar{P}$ & $\mathrm{~K}$ & $\mathrm{Ca}$ & $\mathrm{Mg}$ & $\bar{S}$ & & & $\mathrm{~N}$ & $\mathrm{P}$ & $\mathrm{K}$ & $\mathrm{d}\left(\mathrm{Y}_{\mathrm{N}}\right)$ & $\mathrm{d}\left(\mathrm{Y}_{\mathrm{P}}\right)$ & $\mathrm{d}\left(\mathrm{Y}_{\mathrm{K}}\right)$ \\
\hline & & & & & & & & $\mathrm{tha}^{-1}$ & & & & & na & \\
\hline 000 & -94 & 22 & -111 & 123 & 131 & -71 & 92 & 35,50 & $\mathrm{~V}^{+}$ & $\mathrm{V}^{-}$ & $\mathrm{V}^{+}$ & 1,76 & 2,18 & 0,95 \\
\hline 001 & -88 & 22 & -20 & 104 & 89. & -107 & 72 & 36,45 & $\mathrm{~V}^{+}$ & $\mathrm{V}^{-}$ & $\mathrm{F}^{+}$ & 1,49 & 1,23 & $-0,55$ \\
\hline 002 & -94 & 18 & 35 & 93 & 61. & -114 & 69 & 35,90 & $\mathrm{~V}^{+}$ & $\mathrm{V}^{-}$ & $\mathrm{V}^{-}$ & 1,36 & 0,27 & 1,90 \\
\hline 003 & -108 & 63 & 67 & 88 & 20 & -130 & 79 & 34,00 & $\mathrm{~V}^{+}$ & $\mathrm{F}^{-}$ & - & 1,09 & $-0,82$ & 0,00 \\
\hline 010 & -73 & $32-$ & -108 & 132 & 112 & -95 & 92 & 33,32 & $\mathrm{~V}^{+}$ & $\mathrm{V}^{-}$ & $\mathrm{V}^{+}$ & 2,58 & 3,13 & 1,90 \\
\hline 011 & -88 & 24 & -18 & 108 & 79 . & -105 & 70 & 35,22 & $\mathrm{~V}^{+}$ & $\mathrm{V}^{*}$ & $\mathrm{~V}^{+}$ & 2,45 & 2,17 & 0,41 \\
\hline 012 & -101 & 20 & 42 & 98 & 51. & -111 & 71 & 35,63 & $\mathrm{~V}^{+}$ & $\mathrm{V}^{-}$ & $\mathrm{V}^{-}$ & 2,31 & 0,95 & 0,81 \\
\hline 013 & -126 & 66 & 71 & 95 & 22 & -127 & 85 & 34,82 & $\mathrm{~V}^{+}$ & $\mathrm{V}^{-}$ & - & 2,04 & 0,00 & 0,00 \\
\hline 020 & -51 & $79-$ & -117 & 108 & 92 . & -111 & 93 & 30,19 & $\mathrm{~V}^{+}$ & $\mathrm{V}^{-}$ & $\mathrm{V}^{+}$ & 3,54 & 3,94 & 2,86 \\
\hline 021 & -67 & 21 & -16 & 92 & 77. & -107 & 63 & 33 & $\mathrm{~V}^{+}$ & $\mathrm{V}^{-}$ & $\mathrm{V}^{+}$ &, 40 & 2,86 & 1,63 \\
\hline 022 & -87 & 20 & 41 & 86 & 51 & -111 & 66 & 34,68 & $\mathrm{~V}^{+}$ & $\mathrm{V}^{-}$ & $\mathrm{F}^{-}$ & 3,13 & 1,90 & $-0,14$ \\
\hline 023 & -124 & 65 & 73 & 81 & 34. & -128 & 84 & 34,82 & $\mathrm{~V}^{+}$ & $\mathrm{V}^{-}$ & - & 2,99 & 0,96 & 0,00 \\
\hline 030 & -14 & 115 & -126 & 66 & 94. & -135 & 92 & 26,25 & $\mathrm{~V}^{+}$ & - & $\mathrm{V}^{+}$ & 4,35 & 0,00 & 3,94 \\
\hline 031 & -38 & 61 & -24 & 51 & 79. & -130 & 64 & 30,19 & $\mathrm{~V}^{+}$ & - & $\mathrm{V}^{+}$ & 4,22 & 0,00 & 2,59 \\
\hline 032 & -68 & 58 & 35 & 45 & 65. & -135 & 68 & 32,78 & $\mathrm{~V}^{+}$ & - & $F^{-}$ & 4,08 & 0,00 & $-1,08$ \\
\hline 033 & -116 & 100 & 66 & 42 & 60. & -153 & 90 & & $\mathrm{~V}^{+}$ & - & - & 5 & 0,00 & 0,00 \\
\hline 100 & -71 & $35-$ & -224 & 173 & 181 & -94 & 130 & & $\mathrm{~V}^{+}$ & $\mathrm{V}^{*}$ & $\mathrm{~V}^{+}$ & 0,28 & 1,36 & 0,68 \\
\hline 101 & -60 & $-28-$ & -111 & 142 & 149 & -92 & 97 & 37,94 & $\mathrm{~V}^{+}$ & $\mathrm{F}^{+}$ & $\mathrm{F}^{+}$ & 0,14 & $-0,27$ & $-0,68$ \\
\hline 102 & -62 & 19 & -58 & 112 & 99. & -111 & 77 & 37,26 & $\mathrm{~F}^{+}$ & $\mathrm{F}^{-}$ & $\mathrm{F}^{+}$ & 0,00 & $-0,68$ & $-2,17$ \\
\hline 103 & -55 & 72 & -31 & 100 & 72 & -157 & 81 & 35,09 & $\mathrm{~F}^{+}$ & $F^{*}$ & - & $-0,14$ & $-1,77$ & 0,00 \\
\hline 110 & -53 & $-15-$ & -209 & 188 & 164 & -75 & 117 & 35,90 & $\mathrm{~V}^{+}$ & $\mathrm{F}^{+}$ & $\mathrm{V}^{+}$ & 1,23 & $-2,17$ & 1,77 \\
\hline 111 & -59 & $-25-$ & 101 & 149 & 125 & -89 & 91 & 37,67 & $\mathrm{~V}^{+}$ & $\mathrm{F}^{+}$ & $\mathrm{V}^{+}$ & 1,09 & $-1,22$ & 0,27 \\
\hline 112 & -64 & -30 & -45 & 127 & 107 & -95 & 78 & 37,94 & $\mathrm{~V}^{+}$ & $\mathrm{F}^{+}$ & $\mathrm{F}^{+}$ & 0,96 & $-0,13$ & $-1,08$ \\
\hline 113 & -69 & 28 & -16 & 116 & 79. & -138 & 74 & 36,86 & $\mathrm{~V}^{+}$ & $F^{-}$ & - & 0,81 & $-0,95$ & 0,00 \\
\hline 120 & -27 & $46-$ & -214 & 170 & 147 & -122 & 121 & 33,73 & $\mathrm{~V}^{+}$ & $\mathrm{V}^{-}$ & $\mathrm{V}^{+}$ & 2,17 & 3,13 & 2,72 \\
\hline 121 & -44 & -27 & -97 & 135 & 123 & -91 & 86 & 36,45 & $\mathrm{~V}^{+}$ & $\mathrm{F}^{+}$ & $\mathrm{V}^{+}$ & 2,04 & $-2,04$ & 1,36 \\
\hline 122 & -58 & -30 & -40 & 116 & 106 & -95 & 74 & 37,81 & $\mathrm{~V}^{+}$ & $\mathrm{F}^{+}$ & $\mathrm{F}^{+}$ & 1,77 & $-0,95$ & 0,00 \\
\hline 123 & -72 & 26 & -9 & 106 & 90 & -141 & 74 & 37,81 & $\mathrm{~V}^{+}$ & $\mathrm{V}^{-}$ & - & 1,63 & 0,00 & 0,00 \\
\hline 130 & 9 & & -224 & 127 & 147 & -147 & 124 & 30,60 & $\mathrm{~F}^{-}$ & - & $\mathrm{V}^{+}$ & $-3,13$ & 0,00 & 3,81 \\
\hline 131 & -21 & $18-$ & -104 & 95 & 124 & -113 & 79 & 34,41 & $\mathrm{~V}^{+}$ & - & $\mathrm{V}^{+}$ & 2,85 & 0,00 & 2,45 \\
\hline 132 & -44 & 12 & -44 & 76 & 119 & -119 & 69 & 36,86 & $\mathrm{~V}^{+}$ & - & $\mathrm{V}^{+}$ & 2,72 & 0,00 & 0,95 \\
\hline 133 & -75 & 56 & -19 & 61 & 112 & -136 & 77 & 37,81 & $\mathrm{~V}^{+}$ & - & - & 2,58 & 0,00 & 0,00 \\
\hline
\end{tabular}


Apêndice 20. Índices DRIS, calculados pelo procedimento proposto por Beaufils (1973), diagnose para a correção de $\mathrm{N}, \mathrm{P}$ e K, resposta líquida da produtividade, diagnose para o nutriente deficiente e a resposta da produtividade, para os dados, dos tratamentos com as doses 2 e 3 de $\mathrm{N}$ em todas as combinações de doses de $\mathrm{P}$ e $\mathrm{K}$, do ensaio de adubação $\mathrm{N}, \mathrm{P}$ e $\mathrm{K}$ conduzido em Pirassununga/SP.

\begin{tabular}{|c|c|c|c|c|c|c|c|c|c|c|c|c|c|}
\hline \multirow[b]{2}{*}{ Trat. } & \multicolumn{5}{|c|}{ Indices DRIS } & \multirow[b]{2}{*}{$\mathrm{BN}$} & \multirow[b]{2}{*}{ Prod. } & \multicolumn{3}{|c|}{ Diagnose } & \multicolumn{3}{|c|}{ Resposta } \\
\hline & $\mathrm{N}$ & $\mathrm{K}$ & $\mathrm{Ca}$ & $\mathrm{Mg}$ & $\mathrm{S}$ & & & $\mathrm{N}$ & $\mathrm{P}$ & $\mathrm{K}$ & $\mathrm{d}\left(\mathrm{Y}_{\mathrm{N}}\right)$ & $d\left(Y_{P}\right)$ & $d\left(Y_{K}\right)$ \\
\hline & & & & & & & $\mathrm{tha}^{-1}$ & & & & & t ha & \\
\hline 200 & -55 & $40-290$ & 194 & 200 & -88 & 145 & 37,54 & $\mathrm{~F}^{+}$ & $\mathrm{V}^{-}$ & $\mathrm{V}^{+}$ & $-0,96$ & 0,41 & 0,54 \\
\hline 201 & -34 & $-24-171$ & 151 & 166 & -88 & 106 & 38,08 & $\mathrm{~F}^{+}$ & $\mathrm{V}^{+}$ & $\mathrm{F}^{+}$ & $-1,22$ & 0,68 & $-0,82$ \\
\hline 202 & -32 & $22-120$ & 110 & $128-$ & -108 & 87 & 37,26 & $\mathrm{~F}^{+}$ & $\mathrm{F}^{-}$ & $\mathrm{F}^{+}$ & $-1,36$ & $-1,64$ & $-2,31$ \\
\hline 203 & -16 & $78-102$ & 91 & 101 - & -152 & 90 & 34,95 & $\mathrm{~F}^{+}$ & $\mathrm{F}^{-}$ & - & $-1,49$ & $-2,72$ & 0,00 \\
\hline 210 & -44 & $-10-274$ & 213 & 184 & -69 & 132 & 37,13 & $\mathrm{~F}^{+}$ & $\mathrm{F}^{+}$ & $\mathrm{V}^{+}$ & 0,00 & $-1,23$ & 1,63 \\
\hline 211 & -46 & $-23-161$ & 160 & 155 & -85 & 105 & 38,76 & $\mathrm{~F}^{+}$ & $\mathrm{F}^{+}$ & $\mathrm{V}^{+}$ & $-0,27$ & $-0,27$ & 0,14 \\
\hline 212 & -32 & $\begin{array}{ll}-18 & -97\end{array}$ & 136 & $128-$ & -118 & 88 & 38,90 & $\mathrm{~F}^{+}$ & $\mathrm{V}^{+}$ & $\mathrm{F}^{+}$ & $-0,41$ & 0,68 & $-1,23$ \\
\hline 213 & -32 & $32-81$ & 108 & $108-$ & -135 & 83 & 37,67 & $\mathrm{~F}^{+}$ & $\mathrm{F}^{-}$ & - & $-0,68$ & $-1,77$ & 0,00 \\
\hline 220 & -23 & $50-269$ & 194 & $165-$ & -118 & 137 & 35,90 & $\mathrm{~V}^{+}$ & $\mathrm{V}^{-}$ & $\mathrm{V}^{+}$ & 0,82 & 2,17 & 2,59 \\
\hline 221 & -34 & $-24-155$ & 148 & 153 & -88 & 100 & 38,49 & $\mathrm{~V}^{+}$ & $\mathrm{F}^{+}$ & $\mathrm{V}^{+}$ & 0,68 & $-1,23$ & 1,09 \\
\hline 222 & -36 & $\begin{array}{ll}-28 & -98\end{array}$ & 120 & 135 & -93 & 85 & 39,58 & $\mathrm{~V}^{+}$ & $\mathrm{F}^{+}$ & $\mathrm{F}^{+}$ & 0,54 & 0,00 & $-0,14$ \\
\hline 223 & -43 & $29-74$ & 97 & $130-$ & -139 & 85 & 39,44 & $\mathrm{~V}^{+}$ & $\mathrm{F}^{-}$ & - & 0,27 & $-0,95$ & 0,00 \\
\hline 230 & 6 & $92-270$ & 152 & $163-$ & -144 & 138 & 33,73 & $F^{-}$ & - & $\mathrm{V}^{+}$ & $-1,77$ & 0,00 & 3,53 \\
\hline 231 & -15 & $21-157$ & 109 & $152-$ & -111 & 94 & 37,26 & $\mathrm{~V}^{+}$ & - & $\mathrm{V}^{+}$ & 1,64 & 0,00 & 2,32 \\
\hline 232 & -28 & $16-102$ & 81 & 147 - & -116 & 82 & 39,58 & $\mathrm{~V}^{+}$ & - & $\mathrm{V}^{+}$ & 1,36 & 0,00 & 0,81 \\
\hline 233 & -43 & $14-75$ & 64 & $158-$ & -117 & 79 & 40,39 & $\mathrm{~V}^{+}$ & - & - & 1,23 & 0,00 & 0,00 \\
\hline 300 & -55 & $40-279$ & 182 & 199 & -88 & 141 & 36 , & - & $\mathrm{F}^{-}$ & $\mathrm{V}^{+}$ & 0,00 & $-0,55$ & 0,28 \\
\hline 301 & -30 & $30-186$ & 125 & $161-$ & -100 & 105 & 36,86 & - & $\mathrm{F}^{-}$ & $\mathrm{F}^{+}$ & 0,00 & $-1,63$ & $-0,96$ \\
\hline 302 & -4 & $26-136$ & 86 & $131-$ & -104 & 81 & 35,90 & - & $\mathrm{F}^{-}$ & $\mathrm{F}^{+}$ & 0,00 & $-2,59$ & $-2,44$ \\
\hline 303 & 21 & $83-131$ & 58 & $118-$ & -149 & 93 & 33,46 & - & $\mathrm{F}^{-}$ & - & 0,00 & $-3,53$ & 0,00 \\
\hline 310 & -53 & $43-263$ & 194 & 162 & -84 & 133 & 37,13 & - & $\mathrm{V}^{-}$ & $\mathrm{V}^{+}$ & 0,00 & 0,41 & 1,36 \\
\hline 311 & -29 & $-18-164$ & 147 & 145 & -81 & 97 & 38,49 & - & $\mathrm{V}^{+}$ & $\mathrm{F}^{+}$ & 0,00 & 0,68 & 0,00 \\
\hline 312 & -13 & $-21-117$ & 108 & 128 & -85 & 79 & 38,49 & - & $\mathrm{V}^{+}$ & $\mathrm{F}^{+}$ & 0,00 & 1,63 & $-1,50$ \\
\hline 313 & 0 & $37-108$ & 77 & $125-$ & -131 & 80 & 36,99 & - & $\mathrm{F}^{-}$ & - & 0,00 & $-2,72$ & 0,00 \\
\hline 320 & -36 & $42-253$ & 184 & 147 & -85 & 125 & 36,72 & - & $\mathrm{V}^{-}$ & $\mathrm{V}^{+}$ & 0,00 & 1,22 & 2,45 \\
\hline 321 & -25 & $-20-151$ & 138 & 143 & -84 & 94 & 39,17 & - & $\mathrm{F}^{+}$ & $\mathrm{V}^{+}$ & 0,00 & $-0,27$ & 0,95 \\
\hline 322 & -20 & $-24-107$ & 100 & 138 & -88 & 80 & 40,12 & - & $\mathrm{V}^{+}$ & $\mathrm{F}^{+}$ & 0,00 & 0,82 & $-0,41$ \\
\hline 323 & -21 & $26-97$ & 65 & $131-$ & -104 & 74 & 39,71 & - & $\mathrm{F}^{-}$ & - & 0,00 & $-1,91$ & 0,00 \\
\hline 330 & -6 & $93-247$ & 150 & $151-$ & -142 & 132 & 35,50 & - & - & $\mathrm{V}^{+}$ & 0,00 & 0,00 & 3,40 \\
\hline 331 & -13 & $23-155$ & 99 & $154-$ & -108 & 92 & 38,90 & - & - & $\mathrm{V}^{+}$ & 0,00 & 0,00 & 2,04 \\
\hline 332 & -17 & $19-104$ & 63 & $151-$ & -112 & 78 & 40,94 & - & - & $\mathrm{V}^{+}$ & 0,00 & 0,00 & 0,68 \\
\hline 333 & -17 & $\begin{array}{ll}19 & -93\end{array}$ & 39 & $164-$ & -112 & 74 & 41,62 & - & - & - & 0,00 & 0,00 & 0,00 \\
\hline
\end{tabular}


Apêndice 21. Índices DRIS, calculados pelo procedimento proposto por Elwali \& Gascho (1984), diagnose para a correção de $\mathrm{N}, \mathrm{P}$ e $\mathrm{K}$, resposta líquida da produtividade, diagnose para o nutriente deficiente e a resposta da produtividade, para os dados, dos tratamentos com as doses 0 e 1 de $\mathrm{N}$ em todas as combinações de doses de $\mathrm{P}$ e $\mathrm{K}$, do ensaio de adubação $\mathrm{N}, \mathrm{P}$ e $\mathrm{K}$ conduzido em Araraquara/SP.

\begin{tabular}{|c|c|c|c|c|c|c|c|c|c|c|c|c|c|c|}
\hline \multirow[b]{2}{*}{ Trat. } & \multicolumn{6}{|c|}{ Índices DRIS } & \multirow[b]{2}{*}{$\mathrm{BN}$} & \multirow[b]{2}{*}{ Prod. } & \multicolumn{3}{|c|}{ Diagnose } & \multicolumn{3}{|c|}{ Resposta } \\
\hline & $\mathrm{N}$ & $\mathrm{P}$ & $\mathrm{K}$ & $\mathrm{Ca}$ & $\mathrm{Mg}$ & $\overline{\mathrm{S}}$ & & & $\mathrm{N}$ & $P$ & $\mathrm{~K}$ & $d\left(Y_{N}\right)$ & $\mathrm{d}\left(\mathrm{Y}_{\mathrm{P}}\right)$ & $\mathrm{d}\left(\mathrm{Y}_{\mathrm{K}}\right)$ \\
\hline & & & & & & & & $\mathrm{tha}^{-1}$ & & & & & $\mathrm{tha}^{-1}$ & \\
\hline 000 & 0 & 0 & 20 & -20 & 0 & 0 & 7 & 31,33 & $\mathrm{~F}^{-}$ & $\mathrm{V}^{-}$ & $\mathrm{F}^{-}$ & $-3,64$ & 0,00 & $-2,18$ \\
\hline 001 & -27 & 0 & 87 & -23 & -59 & 21 & 36 & 33,51 & $\mathrm{~V}^{+}$ & $F^{-}$ & $\mathrm{F}^{-}$ & 3,36 & $-0,44$ & $-1,02$ \\
\hline 002 & 10 & 104 & 117 & $-58-$ & -179 & 7 & 79 & 34,53 & $\mathrm{~F}^{-}$ & $F^{-}$ & $\mathrm{V}^{-}$ & $-2,92$ & $-0,88$ & 0,14 \\
\hline 003 & 27 & 125 & 153 & $-34-$ & -293 & 22 & 109 & 34,39 & $\mathrm{~F}^{*}$ & $\mathrm{~F}^{-}$ & - & $-2,48$ & $-1,16$ & 0,00 \\
\hline 010 & 0 & 58 & 21 & -53 & 0 & -26 & 26 & 31,33 & $\mathrm{~F}^{-}$ & $\mathrm{V}^{-}$ & $\mathrm{F}^{-}$ & $-3,35$ & 0,00 & $-2,62$ \\
\hline 011 & 6 & 101 & 92 & $-54-$ & -135 & -11 & 67 & 33,95 & $\mathrm{~F}^{-}$ & $\mathrm{F}^{-}$ & $\mathrm{F}^{-}$ & $-3,06$ & $-0,44$ & $-1,46$ \\
\hline 012 & 11 & 111 & 119 & $-60-$ & -178 & -3 & 80 & 35,41 & $\mathrm{~F}^{-}$ & $F^{-}$ & $\mathrm{F}^{-}$ & $-2,62$ & $-0,73$ & $-0,14$ \\
\hline 013 & 27 & 132 & 182 & $-37-$ & -290 & -14 & 114 & 35,55 & $\mathrm{~F}^{-}$ & $\mathrm{F}^{-}$ & - & $-2,19$ & $-1,17$ & 0,00 \\
\hline 020 & 0 & 65 & 21 & -54 & 0 & -32 & 29 & 31,33 & $\mathrm{~F}^{-}$ & $\mathrm{V}^{-}$ & $\mathrm{F}^{-}$ & $-3,06$ & 0,15 & $-3,06$ \\
\hline 021 & 1 & 98 & 91 & $-56-$ & -101 & -32 & 63 & 34,39 & $\mathrm{~F}^{-}$ & $\mathrm{F}^{-}$ & $\mathrm{F}^{*}$ & $-2,62$ & $-0,14$ & $-1,75$ \\
\hline 022 & 3 & 114 & 144 & $-63-$ & -155 & -44 & 87 & 36,14 & $\mathrm{~F}^{-}$ & $\mathrm{F}^{-}$ & $\mathrm{F}^{-}$ & $-2,33$ & $-0,58$ & $-0,58$ \\
\hline 023 & 14 & 134 & 189 & $-50-$ & -251 & -37 & 113 & 36,72 & $\mathrm{~F}^{-}$ & $\mathrm{F}^{-}$ & - & $-1,89$ & $-1,02$ & 0,00 \\
\hline 030 & -28 & 110 & 23 & -66 & 0 & -38 & 44 & 31,18 & $\mathrm{~V}^{+}$ & - & $\mathrm{F}^{*}$ & 2,63 & 0,00 & $-3,35$ \\
\hline 031 & -29 & 65 & 88 & -60 & -32 & -32 & 51 & 34,53 & $\mathrm{~V}^{+}$ & - & $\mathrm{F}^{-}$ & 2,34 & 0,00 & $-2,19$ \\
\hline 032 & -9 & 112 & 150 & $-69-$ & -118 & -67 & 88 & 36,72 & $\mathrm{~V}^{+}$ & - & $\mathrm{F}^{-}$ & 2,04 & 0,00 & $-1,02$ \\
\hline 033 & -8 & 133 & 195 & $-82-$ & -174 & -64 & 109 & 37,74 & $\mathrm{~V}^{+}$ & - & - & 1,60 & 0,00 & 0,00 \\
\hline 100 & 0 & 0 & 0 & 0 & 0 & 0 & 0 & 34,97 & $\mathrm{~F}^{-}$ & $\mathrm{V}^{-}$ & $\mathrm{F}^{-}$ & $-2,33$ & 0,29 & $-1,90$ \\
\hline 101 & 31 & 26 & 37 & 0 & -68 & -26 & 31 & 36,87 & $\mathrm{~F}^{-}$ & $F^{-}$ & $\mathrm{F}^{-}$ & $-1,89$ & $-0,14$ & $-0,58$ \\
\hline 102 & 17 & 61 & 98 & $-2-$ & -171 & -4 & 59 & 37,45 & $\mathrm{~F}^{-}$ & $F^{-}$ & $\mathrm{V}^{-}$ & $-1,46$ & $-0,58$ & 0,58 \\
\hline 103 & 24 & 71 & 158 & $-2-$ & -225 & -26 & 84 & 36,87 & $F^{-}$ & $F^{-}$ & - & $-1,16$ & $-0,87$ & 0,00 \\
\hline 110 & 28 & 26 & 0 & 0 & -28 & -26 & 18 & 34,68 & $\mathrm{~F}^{-}$ & $\mathrm{V}^{-}$ & $\mathrm{F}^{-}$ & $-2,04$ & 0,29 & $-2,33$ \\
\hline 111 & 37 & 57 & 41 & & -110 & -26 & 45 & 37 , & $\mathrm{F}^{-}$ & $\mathrm{V}^{-}$ & $\mathrm{F}^{-}$ & $-1,46$ & 0,00 & $-1,02$ \\
\hline 112 & 43 & 61 & 74 & $-23-$ & -151 & -4 & 59 & 38,03 & $\mathrm{~F}^{-}$ & $\mathrm{F}^{-}$ & $\mathrm{V}^{-}$ & $-1,17$ & $-0,44$ & 0,29 \\
\hline 113 & 26 & 78 & 164 & $-5-2$ & -221 & -42 & 89 & 37,74 & $\mathrm{~F}^{-}$ & $\mathrm{F}^{*}$ & - & $-0,87$ & $-0,87$ & 0,00 \\
\hline 120 & 29 & 26 & 0 & 0 & -29 & -26 & 18 & 34,39 & $\mathrm{~F}^{-}$ & $\mathrm{V}^{-}$ & $\mathrm{F}^{-}$ & $-1,60$ & 0,58 & $-2,62$ \\
\hline 121 & 32 & 33 & 37 & 0 & -70 & -33 & 34 & 37,01 & $\mathrm{~F}^{-}$ & $\mathrm{V}^{-}$ & $\mathrm{F}^{-}$ & $-1,17$ & 0,14 & $-1,46$ \\
\hline 122 & 17 & 68 & 130 & $-24-$ & -129 & -62 & 72 & 38,47 & $\mathbf{F}^{-}$ & $\mathrm{F}^{-}$ & $\mathrm{F}^{-}$ & $-0,87$ & $-0,29$ & $-0,14$ \\
\hline 123 & 20 & 78 & 171 & $-9-2$ & -217 & -42 & 90 & 38,61 & $\mathrm{~F}^{-}$ & $\mathrm{F}^{-}$ & - & $-0,59$ & $-0,73$ & 0,00 \\
\hline 130 & 0 & 26 & 0 & 0 & 0 & -26 & 9 & 33,81 & $\mathrm{~F}^{-}$ & - & $\mathrm{F}^{-}$ & $-1,31$ & 0,00 & $-3,06$ \\
\hline 131 & 0 & 33 & 54 & -20 & -34 & -33 & 29 & 36,87 & $\mathrm{~F}^{-}$ & - & $\mathrm{F}^{-}$ & $-1,02$ & 0,00 & $-1,89$ \\
\hline 132 & 6 & 64 & 133 & $-27-$ & -113 & -63 & 68 & 38,76 & $\mathrm{~F}^{-}$ & - & $\mathrm{F}^{-}$ & $-0,58$ & 0,00 & $-0,58$ \\
\hline 133 & 21 & 102 & 175 & -84 & -132 & -83 & 100 & 39,34 & $\mathrm{~F}^{-}$ & - & - & $-0,29$ & 0,00 & 0,00 \\
\hline
\end{tabular}


Apêndice 22. Índices DRIS, calculados pelo procedimento proposto por Elwali \& Gascho (1984), diagnose para a correção de $\mathrm{N}, \mathrm{P}$ e K, resposta líquida da produtividade, diagnose para 0 nutriente deficiente e a resposta da produtividade, para os dados, dos tratamentos com as doses 2 e 3 de $\mathrm{N}$ em todas as combinações de doses de $\mathrm{P}$ e $\mathrm{K}$, do ensaio de adubação $\mathrm{N}, \mathrm{P}$ e $\mathrm{K}$ conduzido em Araraquara/SP.

\begin{tabular}{|c|c|c|c|c|c|c|c|c|c|c|c|c|c|c|}
\hline \multirow[b]{2}{*}{ Trat. } & \multicolumn{6}{|c|}{ Indices DRIS } & \multirow[b]{2}{*}{$\mathrm{BN}$} & \multirow[b]{2}{*}{ Prod. } & \multicolumn{3}{|c|}{ Diagnose } & \multicolumn{3}{|c|}{ Resposta } \\
\hline & $\bar{N}$ & $\mathrm{P}$ & $\mathrm{K}$ & $\mathrm{Ca}$ & $\mathrm{Mg}$ & $\bar{S}$ & & & $\mathrm{~N}$ & $\mathrm{P}$ & $\mathrm{K}$ & $\mathrm{d}\left(\mathrm{Y}_{\mathrm{N}}\right)$ & $\mathrm{d}\left(\mathrm{Y}_{\mathrm{P}}\right)$ & $\overline{d\left(Y_{K}\right)}$ \\
\hline & & & & & & & & tha $^{-1}$ & & & & & $\mathrm{tha}^{-1}$ & \\
\hline 200 & 0 & 0 & 0 & 0 & 0 & 0 & 0 & 37,30 & $\mathrm{~F}^{-}$ & $\mathrm{V}^{-}$ & $F^{-}$ & $-0,88$ & 0,58 & $-1,46$ \\
\hline 201 & 29 & 33 & 32 & 0 & -61 & -33 & 31 & 38,76 & $\mathrm{~F}^{-}$ & $\mathrm{V}^{-}$ & $\mathrm{F}^{-}$ & $-0,44$ & 0,29 & $-0,15$ \\
\hline 202 & 34 & 26 & 41 & 0 & -75 & -26 & 34 & 38,91 & $\mathrm{~F}^{-}$ & $\mathrm{F}^{-}$ & $\mathrm{V}^{-}$ & $-0,14$ & $-0,29$ & 0,88 \\
\hline 203 & 14 & 104 & 131 & $-54-$ & -128 & -67 & 83 & 38,03 & $\mathrm{~V}^{*}$ & $\mathrm{~F}^{-}$ & - & 0,29 & $-0,58$ & 0,00 \\
\hline 210 & 31 & 33 & 0 & 0 & -31 & -33 & 21 & 36,72 & $\mathrm{~F}^{-}$ & $\mathrm{V}^{-}$ & $\mathrm{F}^{-}$ & $-0,44$ & 0,73 & $-1,75$ \\
\hline 211 & 35 & 33 & 35 & 0 & -70 & -33 & 34 & 38,47 & $\mathrm{~F}^{-}$ & $\mathrm{V}^{-}$ & $\mathrm{F}^{-}$ & $-0,29$ & 0,29 & $-0,73$ \\
\hline 212 & 40 & 64 & 71 & & -115 & -59 & 58 & 39,20 & $\mathrm{~V}^{*}$ & $\mathrm{~F}^{-}$ & $\mathrm{V}^{-}$ & 0,29 & $-0,14$ & 0,59 \\
\hline 213 & 41 & 68 & 143 & $-49-$ & -136 & -66 & 84 & 38,61 & $\mathrm{~V}^{-}$ & $\mathrm{F}^{-}$ & - & 0,58 & $-0,59$ & 0,00 \\
\hline 220 & 31 & 33 & 0 & 0 & -31 & -33 & 21 & 35,99 & $\mathrm{~F}^{-}$ & $\mathrm{V}^{-}$ & $\mathrm{F}^{-}$ & $-0,15$ & 0,87 & $-2,19$ \\
\hline 221 & 34 & 33 & 34 & 0 & -69 & -33 & 34 & 38,18 & $\mathrm{~V}^{*}$ & $\mathrm{~V}^{-}$ & $\mathrm{F}^{*}$ & 0,15 & 0,29 & $-1,16$ \\
\hline 222 & 39 & 64 & 93 & $-22-$ & -114 & -59 & 65 & 39,34 & $\mathrm{~V}^{-}$ & $\mathrm{V}^{-}$ & $\mathrm{V}^{-}$ & 0,58 & 0,00 & 0,14 \\
\hline 223 & 38 & 76 & 153 & $-53-$ & -134 & -79 & 89 & 39,20 & $\mathrm{~V}^{-}$ & $F^{-}$ & - & 0,88 & $-0,43$ & 0,00 \\
\hline 230 & 29 & 33 & 0 & 0 & -29 & -33 & 21 & 35,12 & $\mathrm{~V}^{-}$ & - & $\mathrm{F}^{-}$ & 0,00 & 0,00 & $-2,77$ \\
\hline 231 & 28 & 33 & 30 & 0 & -58 & -33 & 30 & 37,89 & $\mathrm{~V}^{-}$ & - & $\mathrm{F}^{-}$ & 0,44 & 0,00 & $-1,45$ \\
\hline 232 & 31 & 40 & 96 & -24 & -71 & -72 & 56 & 39,34 & $\mathrm{~V}^{*}$ & - & $\mathrm{F}^{-}$ & 0,87 & 0,00 & $-0,29$ \\
\hline 233 & 25 & 98 & 158 & $-85-$ & -117 & -80 & 94 & 39,63 & $\mathrm{~V}^{*}$ & - & - & 1,16 & 0,00 & 0,00 \\
\hline 300 & 0 & 26 & 0 & 0 & 0 & -26 & 9 & 38,18 & - & $\mathrm{V}^{-}$ & $F^{-}$ & 0,00 & 1,02 & $-1,02$ \\
\hline 301 & 0 & 39 & 0 & 0 & 0 & -39 & 13 & 39,20 & - & $\mathrm{V}^{*}$ & $\mathrm{~V}^{*}$ & 0,00 & 0,44 & 0,15 \\
\hline 302 & 0 & 39 & 0 & 0 & 0 & -39 & 13 & 39,05 & - & $\mathrm{V}^{-}$ & $\mathrm{V}^{-}$ & 0,00 & 0,14 & 1,31 \\
\hline 303 & 22 & 83 & 60 & -87 & -33 & -45 & 55 & 37,74 & - & $\mathrm{F}^{-}$ & - & 0,00 & $-0,29$ & 0,00 \\
\hline 310 & 0 & 33 & 0 & 0 & 0 & -33 & 11 & 37,16 & - & $\mathrm{V}^{-}$ & $F^{-}$ & 0,00 & 1,02 & $-1,60$ \\
\hline 311 & 0 & 39 & 0 & 0 & 0 & -39 & 13 & 38,76 & - & $\mathrm{V}^{-}$ & $\mathrm{F}^{-}$ & 0,00 & 0,73 & $-0,15$ \\
\hline 312 & 0 & 65 & 30 & -26 & -30 & -39 & 32 & 38,91 & - & $\mathrm{V}^{-}$ & $\mathrm{V}^{-}$ & 0,00 & 0,15 & 0,88 \\
\hline 313 & 24 & 71 & 91 & -86 & -35 & -65 & 62 & 38,03 & - & $F^{-}$ & - & 0,00 & $-0,29$ & 0,00 \\
\hline 320 & 31 & 69 & 0 & 0 & -61 & -39 & 33 & 36,14 & - & $\mathrm{V}^{-}$ & $F^{-}$ & 0,00 & 1,02 & $-1,89$ \\
\hline 321 & 29 & 77 & 0 & 0 & -60 & -47 & 36 & 38,03 & - & $\mathrm{V}^{-}$ & $F^{*}$ & 0,00 & 0,58 & $-0,73$ \\
\hline 322 & 0 & 76 & 21 & -50 & 0 & -47 & 32 & 38,76 & - & $\mathrm{V}^{-}$ & $\mathrm{V}^{-}$ & 0,00 & 0,29 & 0,44 \\
\hline 323 & 29 & 113 & 125 & -93 & -97 & -77 & 89 & 38,32 & - & $\mathrm{F}^{-}$ & - & 0,00 & $-0,15$ & 0,00 \\
\hline 330 & 0 & 67 & 0 & -28 & 0 & -39 & 22 & 35,12 & - & - & $\mathrm{F}^{-}$ & 0,00 & 0,00 & $-2,33$ \\
\hline 331 & 0 & 68 & 0 & -29 & 0 & -39 & 23 & 37,45 & - & - & $\mathrm{F}^{-}$ & 0,00 & 0,00 & $-1,02$ \\
\hline 332 & 21 & 80 & 24 & -78 & 0 & -47 & 42 & 38,47 & - & - & $\mathrm{V}^{-}$ & 0,00 & 0,00 & 0,00 \\
\hline 333 & -1 & 87 & 130 & 104 & -35 & -77 & 72 & 38,47 & - & - & - & 0,00 & 0,00 & 0,00 \\
\hline
\end{tabular}


Apêndice 23. Índices DRIS, calculados pelo procedimento proposto por Elwali \& Gascho (1984), diagnose para a correção de $\mathrm{N}, \mathrm{P}$ e $\mathrm{K}$, resposta líquida da produtividade, diagnose para o nutriente deficiente e a resposta da produtividade, para os dados, dos tratamentos com as doses 0 e 1 de $\mathrm{N}$ em todas as combinações de doses de $\mathrm{P}$ e $\mathrm{K}$, do ensaio de adubação $\mathrm{N}, \mathrm{P}$ e $\mathrm{K}$ conduzido em Botucatu/SP.

\begin{tabular}{|c|c|c|c|c|c|c|c|c|c|c|c|c|c|}
\hline \multirow[b]{2}{*}{ Trat. } & \multicolumn{5}{|c|}{ Índices DRIS } & \multirow[b]{2}{*}{$\mathrm{BN}$} & \multirow[b]{2}{*}{ Prod. } & \multicolumn{3}{|c|}{ Diagnose } & \multicolumn{3}{|c|}{ Resposta } \\
\hline & $\overline{\mathrm{N}}$ & $\mathrm{K}$ & $\mathrm{Ca}$ & $\mathrm{Mg}$ & $\bar{S}$ & & & $\mathrm{~N}$ & $\mathrm{P}$ & $\mathrm{K}$ & $\mathrm{d}\left(\mathrm{Y}_{\mathrm{N}}\right)$ & $\mathrm{d}\left(\mathrm{Y}_{\mathrm{P}}\right)$ & $\mathrm{d}\left(\mathrm{Y}_{\mathrm{K}}\right)$ \\
\hline & & & & & & & tha" & & & & & $\mathrm{tha}-1$ & \\
\hline 000 & -97 & $204-728$ & 154 & 477 & -10 & 278 & 36,04 & $\mathrm{~V}^{+}$ & $F^{-}$ & $\mathrm{V}^{+}$ & 4,08 & $-0,76$ & 4,93 \\
\hline 001 & -157 & $113-183$ & 28 & 276 & -76 & 139 & 40,97 & $\mathrm{~V}^{+}$ & $F^{-}$ & $\mathrm{V}^{+}$ & 4,42 & $-0,76$ & 2,29 \\
\hline 002 & -168 & $86 \quad 39$ & -47 & 136 & -46 & 87 & 43,26 & $\mathrm{~V}^{+}$ & $\mathrm{F}^{-}$ & $\mathrm{V}^{-}$ & 4,77 & $-0,69$ & 0,42 \\
\hline 003 & -215 & 62202 - & -182 & 91 & 42 & 132 & 42,84 & $\mathrm{~V}^{+}$ & $F^{-}$ & - & 5,10 & $-0,68$ & 0,00 \\
\hline 010 & -202 & $244-822$ & 202 & 518 & 61 & 342 & 36,80 & $\mathrm{~V}^{+}$ & $F^{-}$ & $\mathrm{V}^{+}$ & 4,51 & $-1,71$ & 4,93 \\
\hline 011 & -261 & $133-243$ & 64 & 302 & 5 & 168 & 41,73 & $\mathrm{~V}^{+}$ & $\mathrm{F}^{-}$ & $\mathrm{V}^{+}$ & 4,85 & $-1,71$ & 2,22 \\
\hline 012 & -261 & $49 \quad 19$ & -2 & 138 & 57 & 88 & 43,95 & $\mathrm{~V}^{+}$ & $F^{-}$ & $\mathrm{V}^{-}$ & 5,27 & $-1,70$ & 0,43 \\
\hline 013 & -270 & 93131 - & -141 & 98 & 89 & 137 & 43,52 & $\mathrm{~V}^{+}$ & $\mathrm{F}^{-}$ & - & 5,52 & $-1,61$ & 0,00 \\
\hline 020 & -245 & $239-925$ & 213 & 560 & 158 & 390 & 38,51 & $\mathrm{~V}^{+}$ & $F^{-}$ & $\mathrm{V}^{+}$ & 5,01 & $-2,72$ & 4,93 \\
\hline 021 & -304 & $129-290$ & 93 & 337 & 34 & 198 & 43,44 & $\mathrm{~V}^{+}$ & $\mathrm{F}^{-}$ & $\mathrm{V}^{+}$ & 5,35 & $-2,72$ & 2,21 \\
\hline 022 & -304 & 89 & 11 & 159 & 40 & 101 & 45,65 & $\mathrm{~V}^{+}$ & $F^{-}$ & $\mathrm{V}^{-}$ & 5,69 & $-2,72$ & 0,52 \\
\hline 023 & -309 & 60113 & -43 & 102 & 77 & 117 & 45,13 & $\mathrm{~V}^{+}$ & $F^{-}$ & - & 6,04 & $-2,72$ & 0,00 \\
\hline 030 & -225 & $277-1154$ & 296 & 635 & 171 & 460 & 41,23 & $\mathrm{~V}^{+}$ & - & $\mathrm{V}^{+}$ & 5,43 & 0,00 & 4,93 \\
\hline 031 & -297 & $131-385$ & 114 & 368 & 70 & 228 & 46,16 & $\mathrm{~V}^{+}$ & - & $\mathrm{V}^{+}$ & 5,78 & 0,00 & 2,21 \\
\hline 032 & -305 & $\begin{array}{ll}68 & -14\end{array}$ & 16 & 229 & 6 & 106 & 48 & $\mathrm{~V}^{+}$ & - & $\mathrm{F}^{+}$ & 6,12 & 0,00 & $-0,52$ \\
\hline 033 & -301 & $57 \quad 75$ & -11 & 107 & 73 & 104 & 47,85 & $\mathrm{~V}^{+}$ & - & - & 6,46 & 0,00 & 0,00 \\
\hline 100 & 16 & $272-1161$ & 238 & 614 & 20 & 387 & 40,12 & $\mathrm{~F}^{-}$ & $F^{-}$ & $\mathrm{V}^{+}$ & $-1,28$ & $-1,19$ & 5,27 \\
\hline 101 & -82 & $124-395$ & 81 & 356 & -83 & 187 & 45,39 & $\mathrm{~V}^{+}$ & $F^{-}$ & $\mathrm{V}^{+}$ & 1,70 & $-1,19$ & 2,64 \\
\hline 102 & -89 & $\begin{array}{ll}49 & -43\end{array}$ & -53 & 220 & -84 & 90 & 48,03 & $\mathrm{~V}^{+}$ & $F^{-}$ & $\mathrm{F}^{+}$ & 2,03 & $-1,19$ & $-0,09$ \\
\hline 103 & -144 & 110 & -129 & 100 & -6 & 93 & 47,94 & $\mathrm{~V}^{+}$ & $F^{-}$ & - & 2,38 & $-1,10$ & 0,00 \\
\hline 110 & -37 & $313-1239$ & 283 & 621 & 59 & 425 & 41,31 & $\mathrm{~V}^{+}$ & $F^{-}$ & $\mathrm{V}^{+}$ & 1,78 & $-2,21$ & 5,27 \\
\hline 111 & -133 & $155-440$ & 118 & 358 & -58 & 210 & 46,58 & $\mathrm{~V}^{+}$ & $F^{-}$ & $\mathrm{V}^{+}$ & 2,13 & $-2,21$ & 2,64 \\
\hline 112 & -174 & $119-104$ & -15 & 204 & -30 & 108 & 49,22 & $\mathrm{~V}^{+}$ & $F^{-}$ & $\mathrm{F}^{+}$ & 2,37 & $-2,12$ & $-0,18$ \\
\hline 113 & -170 & $91 \quad 41$ & -79 & 106 & 11 & 83 & 49,04 & $\mathrm{~V}^{+}$ & $F^{*}$ & - & 2,81 & $-2,13$ & 0,00 \\
\hline 120 & -76 & $323-1376$ & 332 & 666 & 131 & 484 & 43,52 & $\mathrm{~V}^{+}$ & $F^{-}$ & $\mathrm{V}^{+}$ & 2,21 & $-3,14$ & 5,27 \\
\hline 121 & -188 & $201-521$ & 148 & 378 & -19 & 243 & 48,79 & $\mathrm{~V}^{+}$ & $F^{-}$ & $\mathrm{V}^{+}$ & 2,55 & $-3,15$ & 2,55 \\
\hline 122 & -193 & $101-150$ & 28 & 243 & -29 & 124 & 51,34 & $\mathrm{~V}^{+}$ & $F^{-}$ & $\mathrm{F}^{+}$ & 2,89 & $-3,15$ & $-0,17$ \\
\hline 123 & -192 & $110 \quad 38$ & -73 & 104 & 13 & 88 & 51,17 & $\mathrm{~V}^{+}$ & $F^{-}$ & - & 3,32 & $-3,14$ & 0,00 \\
\hline 130 & -10 & $451-1726$ & 411 & 769 & 106 & 579 & 46,66 & $\mathrm{~V}^{+}$ & - & $\mathrm{V}^{+}$ & 2,64 & 0,00 & 5,28 \\
\hline 131 & -144 & $225-609$ & 182 & 410 & -63 & 272 & 51,94 & $\mathrm{~V}^{+}$ & - & $\mathrm{V}^{+}$ & 2,97 & 0,00 & 2,55 \\
\hline 132 & -206 & $166-199$ & 43 & 235 & -39 & 148 & 54,49 & $\mathrm{~V}^{+}$ & - & $\mathrm{F}^{+}$ & 3,31 & 0,00 & $-0,18$ \\
\hline 133 & -172 & 89 & -45 & 124 & -4 & 74 & 54,31 & $\mathrm{~V}^{+}$ & - & - & 3,66 & 0,00 & 0,00 \\
\hline
\end{tabular}


Apêndice 24. Índices DRIS, calculados pelo procedimento proposto por Elwali \& Gascho (1984), diagnose para a correção de $\mathrm{N}, \mathrm{P}$ e $\mathrm{K}$, resposta líquida da produtividade, diagnose para o nutriente deficiente e a resposta da produtividade, para os dados, dos tratamentos com as doses 2 e 3 de $\mathrm{N}$ em todas as combinações de doses de $\mathrm{P}$ e $\mathrm{K}$, do ensaio de adubação $\mathrm{N}, \mathrm{P}$ e $\mathrm{K}$ conduzido em Botucatu/SP.

\begin{tabular}{|c|c|c|c|c|c|c|c|c|c|c|c|c|c|}
\hline \multirow[b]{2}{*}{ Trat. } & \multicolumn{5}{|c|}{ Indices DRIS } & \multirow[b]{2}{*}{$\mathrm{BN}$} & \multirow[b]{2}{*}{ Prod. } & \multicolumn{3}{|c|}{ Diagnose } & \multicolumn{3}{|c|}{ Resposta } \\
\hline & $\overline{\mathrm{N}}$ & $\mathrm{K}$ & $\mathrm{Ca}$ & $\mathrm{Mg}$ & $S$ & & & $\mathrm{~N}$ & $P$ & $\mathrm{~K}$ & $\mathrm{~d}\left(\mathrm{Y}_{\mathrm{N}}\right)$ & $\mathrm{d}\left(\mathrm{Y}_{\mathrm{P}}\right)$ & $d\left(Y_{K}\right)$ \\
\hline & & & & & & & $\mathrm{tha}^{-1}$ & & & & & $\mathrm{tha}^{-1}$ & \\
\hline 200 & 104 & $332-1513$ & 311 & 735 & 31 & 504 & 41,40 & $\mathrm{~V}^{-}$ & $\mathrm{F}^{-}$ & $\mathrm{V}^{+}$ & 1,45 & $-1,69$ & 5,69 \\
\hline 201 & -11 & $129-576$ & 115 & 440 & -97 & 228 & 47,09 & $\mathrm{~F}^{+}$ & $F^{-}$ & $\mathrm{V}^{+}$ & $-1,19$ & $-1,62$ & 2,97 \\
\hline 202 & -70 & $127-207$ & -31 & $286-$ & -105 & 138 & 50,06 & $\mathrm{~F}^{+}$ & $F^{-}$ & $\mathrm{V}^{+}$ & $-0,84$ & $-1,53$ & 0,26 \\
\hline 203 & -76 & $108-28$ & -104 & 189 & -89 & 99 & 50,32 & $\mathrm{~F}^{+}$ & $F^{-}$ & - & $-0,51$ & $-1,53$ & 0,00 \\
\hline 210 & 40 & $373-1522$ & 346 & 734 & 29 & 507 & 43,09 & $\mathrm{~V}^{-}$ & $F^{-}$ & $\mathrm{V}^{+}$ & 1,10 & $-2,64$ & 5,62 \\
\hline 211 & -86 & $241-636$ & 153 & $440-$ & -113 & 278 & 48,71 & $\mathrm{~F}^{+}$ & $F^{-}$ & $\mathrm{V}^{+}$ & $-0,68$ & $-2,63$ & 2,88 \\
\hline 212 & -118 & $183-240$ & 14 & $260-$ & -100 & 153 & 51,59 & $\mathrm{~F}^{+}$ & $F^{-}$ & $\mathrm{V}^{+}$ & $-0,33$ & $-2,64$ & 0,26 \\
\hline 213 & -95 & $115-31$ & -89 & 180 & -81 & 99 & 51,85 & $\mathrm{~F}^{+}$ & $F^{-}$ & - & 0,00 & $-2,64$ & 0,00 \\
\hline 220 & 36 & $462-1706$ & 405 & 773 & 30 & 569 & 45,73 & $\mathrm{~V}^{-}$ & $F^{-}$ & $\mathrm{V}^{+}$ & 0,60 & $-3,57$ & 5,61 \\
\hline 221 & -92 & $252-685$ & 191 & $445-$ & -111 & 296 & 51,34 & $\mathrm{~F}^{+}$ & $\mathrm{F}^{-}$ & $\mathrm{V}^{+}$ & $-0,25$ & $-3,57$ & 2,89 \\
\hline 222 & -125 & $188-263$ & 65 & $258-$ & -124 & 171 & 54,23 & $\mathrm{~V}^{+}$ & $F^{-}$ & $\mathrm{V}^{+}$ & 0,08 & $-3,57$ & 0,26 \\
\hline 223 & -105 & $182-66$ & -81 & $178-$ & -108 & 120 & 54,49 & $\mathrm{~V}^{+}$ & $\mathrm{F}^{-}$ & - & 0,42 & $-3,48$ & 0,00 \\
\hline 230 & 100 & $544-2038$ & 492 & 861 & 42 & 680 & 49,30 & $\mathrm{~V}^{-}$ & - & $\mathrm{V}^{+}$ & 0,08 & 0,00 & 5,61 \\
\hline 231 & -81 & $334-794$ & 226 & $463-$ & -149 & 341 & 54,91 & $\mathrm{~V}^{+}$ & - & $\mathrm{V}^{+}$ & 0,25 & 0,00 & 2,89 \\
\hline 232 & -121 & $258-312$ & 93 & $274-$ & -192 & 208 & 57,80 & $\mathrm{~V}^{+}$ & - & $\mathrm{V}^{+}$ & 0,60 & 0,00 & 0,17 \\
\hline 233 & -101 & $193-76$ & -76 & $182-$ & -121 & 125 & 57 & $\mathrm{~V}^{+}$ & - & - & 0,94 & 0,00 & 0,00 \\
\hline 300 & 184 & $347-1571$ & 343 & $843-$ & -146 & 572 & 39,95 & - & $F^{-}$ & $\mathrm{V}^{+}$ & 0,00 & $-2,04$ & 5,95 \\
\hline 301 & 27 & $248-674$ & 148 & 528 & -277 & 317 & 45,90 & - & $F^{-}$ & $\mathrm{V}^{+}$ & 0,00 & $-2,13$ & 3,32 \\
\hline 302 & -10 & $167-268$ & 13 & 367 & -269 & 182 & 49,22 & - & $F^{-}$ & $\mathrm{V}^{+}$ & 0,00 & $-2,04$ & 0,59 \\
\hline 303 & -89 & $185-83$ & -120 & $252-$ & -146 & 146 & 49,81 & - & $\mathrm{F}^{-}$ & - & 0,00 & $-2,04$ & 0,00 \\
\hline 310 & 76 & $402-1497$ & 363 & $787-$ & -131 & 543 & 41,99 & - & $F^{-}$ & $\mathrm{V}^{+}$ & 0,00 & $-3,14$ & 6,04 \\
\hline 311 & -28 & $286-688$ & 183 & $500-$ & -252 & 323 & 48,03 & - & $F^{-}$ & $\mathrm{V}^{+}$ & 0,00 & $-3,06$ & 3,23 \\
\hline 312 & -47 & $202-300$ & 35 & $343-$ & -233 & 193 & 51,26 & - & $F^{-}$ & $\mathrm{V}^{+}$ & 0,00 & $-3,05$ & 0,59 \\
\hline 313 & -104 & $209-91$ & -104 & $235-$ & -146 & 148 & 51,85 & - & $F^{-}$ & - & 0,00 & $-3,06$ & 0,00 \\
\hline 320 & 72 & $496-1583$ & 415 & $803-$ & -203 & 595 & 45,13 & - & $F^{-}$ & $\mathrm{V}^{+}$ & 0,00 & $-4,09$ & 5,96 \\
\hline 321 & -34 & $369-748$ & 226 & $511-$ & -325 & 369 & 51,09 & - & $F^{-}$ & $\mathrm{V}^{+}$ & 0,00 & $-4,07$ & 3,22 \\
\hline 322 & -72 & $287-312$ & 91 & 325 & -319 & 234 & 54,31 & - & $F^{-}$ & $\mathrm{V}^{+}$ & 0,00 & $-4,09$ & 0,60 \\
\hline 323 & -111 & $261-104$ & -95 & $226-$ & -176 & 162 & 54,91 & - & $F^{-}$ & - & 0,00 & $-4,00$ & 0,00 \\
\hline 330 & 138 & $650-1801$ & 488 & $857-$ & -332 & 711 & 49,22 & - & - & $\mathrm{V}^{+}$ & 0,00 & 0,00 & 5,94 \\
\hline 331 & 10 & $485-824$ & 271 & 529 & -470 & 432 & 55,16 & - & - & $\mathrm{V}^{+}$ & 0,00 & 0,00 & 3,24 \\
\hline 332 & -36 & $376-359$ & 126 & 326 & -433 & 276 & 58,40 & - & - & $\mathrm{V}^{+}$ & 0,00 & 0,00 & 0,51 \\
\hline 333 & -67 & $291-115$ & -56 & $229-$ & -282 & 173 & 58,91 & - & - & - & 0,00 & 0,00 & 0,00 \\
\hline
\end{tabular}


Apêndice 25. Índices DRIS, calculados pelo procedimento proposto por Elwali \& Gascho (1984), diagnose para a correção de $\mathrm{N}, \mathrm{P}$ e K, resposta líquida da produtividade, diagnose para o nutriente deficiente e a resposta da produtividade, para os dados, dos tratamentos com as doses 0 e 1 de $\mathrm{N}$ em todas as combinações de doses de $\mathrm{P}$ e $\mathrm{K}$, do ensaio de adubação $\mathrm{N}, \mathrm{P}$ e $\mathrm{K}$ conduzido em Matão/SP.

\begin{tabular}{|c|c|c|c|c|c|c|c|c|c|c|c|c|c|c|}
\hline \multirow[b]{2}{*}{ Trat. } & \multicolumn{6}{|c|}{ Índices DRIS } & \multirow[b]{2}{*}{ BN } & \multirow[b]{2}{*}{ Prod. } & \multicolumn{3}{|c|}{ Diagnose } & \multicolumn{3}{|c|}{ Resposta } \\
\hline & $\bar{N}$ & $\mathbf{P}$ & $\mathrm{K}$ & $\mathrm{Ca}$ & $\mathrm{Mg}$ & $\mathrm{S}$ & & & $\mathrm{N}$ & $P$ & $\mathrm{~K}$ & $d\left(Y_{N}\right)$ & $d\left(Y_{P}\right)$ & $\mathrm{d}\left(\mathrm{Y}_{\mathrm{K}}\right)$ \\
\hline & & & & & & & & $\mathrm{tha}^{-1}$ & & & & & $-\mathrm{tha}^{-1}$ & \\
\hline 000 & -62 & 0 & 28 & 0 & 34 & 0 & 21 & 34,77 & $\mathrm{~V}^{+}$ & $\mathrm{F}^{-}$ & $\mathrm{V}^{-}$ & 2,21 & $-4,75$ & 1,54 \\
\hline 001 & -75 & -20 & 99 & 0 & 29 & -32 & 43 & 33,23 & $\mathrm{~V}^{+}$ & $\mathrm{V}^{+}$ & $\mathrm{F}^{-}$ & 2,04 & 4,34 & $-0,35$ \\
\hline 002 & -63 & -6 & 181 & -33 & 0 & -78 & 60 & 33,58 & $\mathrm{~V}^{+}$ & $\mathrm{V}^{+}$ & $\mathrm{F}^{-}$ & 1,95 & 3,90 & $-2,21$ \\
\hline 003 & -78 & 0 & 279 & -49 & -32 & -119 & 93 & 35,79 & $\mathrm{~V}^{+}$ & $\mathrm{F}^{-}$ & - & 1,86 & $-3,48$ & 0,00 \\
\hline 010 & -72 & 26 & 36 & -27 & 63 & -26 & 42 & 39,52 & $\mathrm{~V}^{+}$ & $F^{-}$ & $\mathrm{V}^{-}$ & 2,30 & $-2,47$ & 1,95 \\
\hline 011 & -76 & 3 & 111 & 0 & 27 & -66 & 47 & 37,57 & $\mathrm{~V}^{+}$ & $\mathrm{F}^{-}$ & $\mathrm{V}^{-}$ & 2,13 & $-2,04$ & 0,09 \\
\hline 012 & -81 & 15 & 168 & -31 & 21 & -92 & 68 & 37,48 & $\mathrm{~V}^{+}$ & $F^{-}$ & $F^{*}$ & 2,04 & $-1,54$ & $-1,79$ \\
\hline 013 & -71 & 25 & 219 & -44 & & -130 & 82 & 39,27 & $\mathrm{~V}^{+}$ & $F^{-}$ & - & 1,96 & $-1,11$ & 0,00 \\
\hline 020 & -72 & 26 & 61 & 0 & 36 & -51 & 41 & 41,99 & $\mathrm{~V}^{+}$ & $F^{-}$ & $\mathrm{V}^{-}$ & 2,38 & $-0,09$ & 2,38 \\
\hline 021 & -74 & -21 & 98 & 0 & 29 & -32 & 42 & 39,61 & $\mathrm{~V}^{+}$ & $\mathrm{F}^{+}$ & $\mathrm{V}^{-}$ & 2,21 & $-0,34$ & 0,59 \\
\hline 022 & -72 & 32 & 115 & -24 & 22 & -73 & 56 & 39,02 & $\mathrm{~V}^{+}$ & $\mathrm{V}^{-}$ & $\mathrm{F}^{-}$ & 2,12 & 0,77 & $-1,36$ \\
\hline 023 & -56 & 24 & 170 & -34 & & -104 & 65 & 40,38 & $\mathrm{~V}^{+}$ & $\mathrm{V}^{-}$ & - & 2,03 & 1,19 & 0,00 \\
\hline 030 & -94 & -23 & 30 & 25 & 62 & 0 & 39 & 42,08 & $\mathrm{~V}^{+}$ & - & $\mathrm{V}^{-}$ & 2,54 & 0,00 & 2,81 \\
\hline 031 & -64 & 0 & 33 & 0 & 31 & 0 & 21 & 39,27 & $\mathrm{~V}^{+}$ & - & $\mathrm{V}^{-}$ & 2,29 & 0,00 & 1,02 \\
\hline 032 & -58 & 0 & 36 & 0 & 23 & 0 & 20 & 38,25 & $\mathrm{~V}^{+}$ & - & $\mathrm{F}^{-}$ & 2,29 & 0,00 & $-0,94$ \\
\hline 033 & -35 & 0 & 64 & 0 & 0 & -29 & 21 & 39,19 & $\mathrm{~V}^{+}$ & - & - & 2,12 & 0,00 & 0,00 \\
\hline 100 & -28 & 33 & 0 & -30 & 88 & -63 & 40 & 36,98 & $\mathrm{~V}^{+}$ & $F^{-}$ & $\mathrm{V}^{-}$ & 1,01 & $-4,84$ & 1,71 \\
\hline 101 & -23 & 26 & 0 & -30 & 53 & -26 & 26 & 35,27 & $\mathrm{~V}^{+}$ & $F^{*}$ & $\mathrm{~F}^{-}$ & 0,94 & $-4,43$ & $-0,26$ \\
\hline 102 & -38 & 4 & 123 & -24 & 0 & -65 & 42 & 35,53 & $\mathrm{~V}^{+}$ & $F^{-}$ & $\mathrm{F}^{-}$ & 0,85 & $-3,99$ & $-2,12$ \\
\hline 103 & -54 & 8 & 191 & -39 & & -106 & 66 & 37,65 & $\mathrm{~V}^{+}$ & $\mathrm{F}^{-}$ & - & 0,69 & $-3,58$ & 0,00 \\
\hline 110 & -28 & 40 & 0 & -29 & 91 & -74 & 44 & 41,82 & $\mathrm{~V}^{+}$ & $F^{-}$ & $\mathrm{V}^{-}$ & 1,19 & $-2,55$ & 2,12 \\
\hline 111 & -49 & 26 & 55 & -28 & 51 & -54 & 44 & 39,70 & $\mathrm{~V}^{+}$ & $F^{-}$ & $\mathrm{V}^{-}$ & 1,10 & $-2,12$ & 0,18 \\
\hline 112 & -39 & 47 & 111 & -51 & 27 & -94 & 62 & 39,52 & $\mathrm{~V}^{+}$ & $F^{-}$ & $F^{-}$ & 0,94 & $-1,62$ & $-1,71$ \\
\hline 113 & -51 & 9 & 184 & -37 & & -105 & 64 & 41,23 & $\mathrm{~V}^{+}$ & $\mathrm{F}^{*}$ & - & 0,76 & $-1,18$ & 0,00 \\
\hline 120 & -28 & 33 & 0 & 0 & 59 & -63 & 31 & 44,37 & $\mathrm{~V}^{+}$ & $F^{-}$ & $\mathrm{V}^{-}$ & 1,28 & $-0,25$ & 2,55 \\
\hline 121 & -48 & 26 & 54 & 0 & 22 & -54 & 34 & 41,82 & $\mathrm{~V}^{+}$ & $\mathrm{V}^{-}$ & $\mathrm{V}^{-}$ & 1,19 & 0,26 & 0,68 \\
\hline 122 & -34 & 39 & 73 & 0 & 0 & -77 & 37 & 41,14 & $\mathrm{~V}^{+}$ & $\mathrm{V}^{-}$ & $\mathrm{F}^{-}$ & 1,10 & 0,60 & $-1,27$ \\
\hline 123 & -42 & 47 & 122 & -29 & 0 & -98 & 56 & 42,41 & $\mathrm{~V}^{+}$ & $\mathrm{V}^{-}$ & - & 0,94 & 1,10 & 0,00 \\
\hline 130 & -28 & -22 & 0 & 0 & 50 & 0 & 17 & 44,62 & $\mathrm{~V}^{+}$ & - & $\mathrm{V}^{-}$ & 1,37 & 0,00 & 3,06 \\
\hline 131 & -24 & 0 & 0 & 0 & 24 & 0 & 8 & 41,56 & $\mathrm{~V}^{+}$ & - & $\mathrm{V}^{-}$ & 1,28 & 0,00 & 1,02 \\
\hline 132 & 0 & 0 & 0 & 0 & 0 & 0 & 0 & 40,54 & $\mathrm{~F}^{-}$ & - & $\mathrm{F}^{-}$ & $-1,11$ & 0,00 & $-0,77$ \\
\hline 133 & -26 & 0 & 53 & 0 & 0 & -27 & 18 & 41,31 & $\mathrm{~V}^{+}$ & - & - & 1,02 & 0,00 & 0,00 \\
\hline
\end{tabular}


Apêndice 26. Índices DRIS, calculados pelo procedimento proposto por Elwali \& Gascho (1984), diagnose para a correção de $\mathrm{N}, \mathrm{P}$ e $\mathrm{K}$, resposta líquida da produtividade, diagnose para $\mathrm{o}$ nutriente deficiente e a resposta da produtividade, para os dados, dos tratamentos com as doses 2 e 3 de $\mathrm{N}$ em todas as combinações de doses de $\mathrm{P}$ e $\mathrm{K}$, do ensaio de adubação $\mathrm{N}, \mathrm{P}$ e $\mathrm{K}$ conduzido em Matão/SP.

\begin{tabular}{|c|c|c|c|c|c|c|c|c|c|c|c|c|c|c|}
\hline \multirow[b]{2}{*}{ Trat. } & \multicolumn{6}{|c|}{ Índices DRIS } & \multirow[b]{2}{*}{$\mathrm{BN}$} & \multirow[b]{2}{*}{ Prod. } & \multicolumn{3}{|c|}{ Diagnose } & \multicolumn{3}{|c|}{ Resposta } \\
\hline & $\mathrm{N}$ & $P$ & $\mathrm{~K}$ & $\mathrm{Ca}$ & $\mathrm{Mg}$ & $\mathrm{S}$ & & & $\mathrm{N}$ & $\mathrm{P}$ & $\mathrm{K}$ & $\mathrm{d}\left(\mathrm{Y}_{\mathrm{N}}\right)$ & $\mathrm{d}\left(\mathrm{Y}_{\mathrm{P}}\right)$ & $\mathrm{d}\left(\mathrm{Y}_{\mathrm{K}}\right)$ \\
\hline & & & & & & & & $\mathrm{tha}^{-1}$ & & & & & $-t h a^{-1}$ & \\
\hline 200 & -21 & 33 & -27 & -32 & 110 & -63 & 48 & 37,99 & $\mathrm{~F}^{+}$ & $\mathrm{F}^{-}$ & $\mathrm{F}^{+}$ & 0,00 & $-5,02$ & $-1,78$ \\
\hline 201 & 0 & 26 & 0 & -30 & 30 & -26 & 19 & 36,21 & $\mathrm{~V}^{-}$ & $F^{-}$ & $\mathrm{F}^{-}$ & 0,09 & $-4,59$ & $-0,17$ \\
\hline 202 & -3 & 33 & 85 & -49 & 28 & -94 & 49 & 36,38 & $\mathrm{~F}^{+}$ & $F^{-}$ & $\mathrm{F}^{-}$ & $-0,26$ & $-4,08$ & $-1,96$ \\
\hline 203 & -16 & -12 & 176 & -36 & & -113 & 59 & 38,34 & $\mathrm{~F}^{+}$ & $\mathrm{V}^{+}$ & - & $-0,35$ & 3,65 & 0,00 \\
\hline 210 & 26 & 40 & 0 & -29 & 61 & -98 & 42 & 43,01 & $\mathrm{~F}^{-}$ & $F^{-}$ & $\mathrm{V}^{-}$ & $-0,08$ & $-2,64$ & 2,21 \\
\hline 211 & 23 & 33 & 29 & -30 & 30 & -84 & 38 & 40,80 & $\mathrm{~V}^{-}$ & $F^{-}$ & $\mathrm{V}^{-}$ & 0,00 & $-2,21$ & 0,34 \\
\hline 212 & -6 & 11 & 119 & -50 & 27 & -101 & 52 & 40,46 & $\mathrm{~F}^{+}$ & $F^{-}$ & $\mathrm{F}^{-}$ & $-0,17$ & $-1,78$ & $-1,53$ \\
\hline 213 & -15 & 10 & 173 & -36 & & -132 & 61 & 41,99 & $\mathrm{~F}^{+}$ & $\mathrm{F}^{-}$ & - & $-0,26$ & $-1,36$ & 0,00 \\
\hline 220 & 0 & 33 & 0 & 0 & 0 & -33 & 11 & 45,65 & $\mathrm{~F}^{-}$ & $F^{*}$ & $\mathrm{~V}^{-}$ & $-0,16$ & $-0,34$ & 2,64 \\
\hline 221 & 0 & 26 & 28 & 0 & 0 & -53 & 18 & 43,01 & $\mathrm{~F}^{-}$ & $\mathrm{V}^{-}$ & $\mathrm{V}^{-}$ & $-0,08$ & 0,17 & 0,77 \\
\hline 222 & -28 & 4 & 110 & -21 & 0 & -65 & 38 & 42,24 & $\mathrm{~F}^{+}$ & $\mathrm{V}^{-}$ & $F^{-}$ & $-0,08$ & 0,59 & $-1,11$ \\
\hline 223 & -14 & 5 & 148 & -31 & & -108 & 51 & 43,35 & $\mathrm{~F}^{+}$ & $\mathrm{V}^{-}$ & - & $-0,17$ & 1,02 & 0,00 \\
\hline 230 & 0 & 0 & 0 & 0 & 0 & 0 & 0 & 45,99 & $\mathrm{~F}^{-}$ & - & $\mathrm{V}^{-}$ & $-0,25$ & 0,00 & 3,15 \\
\hline 231 & 0 & 0 & 0 & 0 & 0 & 0 & 0 & 42,84 & $\mathrm{~F}^{-}$ & - & $\mathrm{V}^{-}$ & $-0,17$ & 0,00 & 1,19 \\
\hline 232 & 0 & 0 & 0 & 0 & 0 & 0 & 0 & 41,65 & $\mathrm{~V}^{-}$ & - & $\mathrm{F}^{-}$ & 0,00 & 0,00 & $-0,68$ \\
\hline 233 & -25 & 0 & 76 & -20 & 0 & -31 & 25 & 42,33 & $\mathrm{~F}^{+}$ & - & - & $-0,09$ & 0,00 & 0,00 \\
\hline 300 & 54 & 33 & -49 & -32 & 56 & -62 & 48 & 37,99 & - & $\mathrm{F}^{-}$ & $\mathrm{F}^{+}$ & 0,00 & $-5,10$ & $-1,87$ \\
\hline 301 & 24 & 26 & 0 & -32 & 32 & -49 & 27 & 36,12 & - & $\mathrm{F}^{-}$ & $\mathrm{V}^{-}$ & 0,00 & $-4,68$ & 0,00 \\
\hline 302 & 23 & -28 & 86 & -50 & 28 & -59 & 46 & 36,12 & - & $\mathrm{V}^{+}$ & $\mathrm{F}^{-}$ & 0,00 & 4,17 & $-1,87$ \\
\hline 303 & -13 & -41 & 177 & -38 & 0 & -85 & 59 & 37,99 & - & $\mathrm{V}^{+}$ & - & 0,00 & 3,74 & 0,00 \\
\hline 310 & 27 & 33 & 0 & -29 & 29 & -60 & 30 & 43,09 & - & $\mathrm{F}^{-}$ & $\mathrm{V}^{-}$ & 0,00 & $-2,72$ & 2,29 \\
\hline 311 & 23 & 26 & 28 & -29 & 29 & -77 & 35 & 40,80 & - & $\mathrm{F}^{-}$ & $\mathrm{V}^{-}$ & 0,00 & $-2,29$ & 0,51 \\
\hline 312 & -6 & -1 & 128 & -28 & 0 & -94 & 43 & 40,29 & - & $\mathrm{V}^{+}$ & $F^{-}$ & 0,00 & 1,87 & $-1,44$ \\
\hline 313 & -16 & -45 & 193 & -40 & 0 & -92 & 64 & 41,73 & - & $\mathrm{V}^{+}$ & - & 0,00 & 1,45 & 0,00 \\
\hline 320 & 26 & 33 & 0 & 0 & 0 & -58 & 20 & 45,81 & - & $F^{-}$ & $\mathrm{V}^{-}$ & 0,00 & $-0,43$ & 2,72 \\
\hline 321 & 0 & 0 & 29 & 0 & 0 & -29 & 10 & 43,09 & - & $\mathrm{V}^{-}$ & $\mathrm{V}^{-}$ & 0,00 & 0,08 & 0,93 \\
\hline 322 & -31 & -27 & 127 & -27 & 0 & -41 & 42 & 42,16 & - & $\mathrm{F}^{+}$ & $F^{-}$ & 0,00 & $-0,51$ & $-1,02$ \\
\hline 323 & -20 & -44 & 178 & -37 & 0 & -77 & 59 & 43,18 & - & $\mathrm{F}^{+}$ & - & 0,00 & $-0,94$ & 0,00 \\
\hline 330 & 0 & 0 & 0 & 0 & 0 & 0 & 0 & 46,24 & - & - & $\mathrm{V}^{-}$ & 0,00 & 0,00 & 3,23 \\
\hline 331 & 0 & -27 & 27 & 0 & 0 & 0 & 9 & 43,01 & - & - & $\mathrm{V}^{-}$ & 0,00 & 0,00 & 1,36 \\
\hline 332 & -27 & -33 & 110 & -22 & 0 & -28 & 37 & 41,65 & - & - & $F^{-}$ & 0,00 & 0,00 & $-0,59$ \\
\hline 333 & -33 & -38 & 133 & -29 & 0 & -33 & 44 & 42,24 & - & - & - & 0,00 & 0,00 & 0,00 \\
\hline
\end{tabular}


Apêndice 27. Índices DRIS, calculados pelo procedimento proposto por Elwali \& Gascho (1984), diagnose para a correção de $\mathrm{N}, \mathrm{P}$ e $\mathrm{K}$, resposta líquida da produtividade, diagnose para o nutriente deficiente e a resposta da produtividade, para os dados, dos tratamentos com as doses 0 e 1 de $\mathrm{N}$ em todas as combinações de doses de $\mathrm{P}$ e $\mathrm{K}$, do ensaio de adubação $\mathrm{N}, \mathrm{P}$ e $\mathrm{K}$ conduzido em Monte Azul/SP.

\begin{tabular}{|c|c|c|c|c|c|c|c|c|c|c|c|c|c|c|}
\hline \multirow[b]{2}{*}{ Trat. } & \multicolumn{6}{|c|}{ Índices DRIS } & \multirow[b]{2}{*}{$\mathrm{BN}$} & \multirow[b]{2}{*}{ Prod. } & \multicolumn{3}{|c|}{ Diagnose } & \multicolumn{3}{|c|}{ Resposta } \\
\hline & $\overline{\mathrm{N}}$ & $P$ & $\mathrm{~K}$ & $\mathrm{Ca}$ & $\mathrm{Mg}$ & $S$ & & & $\mathrm{~N}$ & $P$ & $\mathrm{~K}$ & $\mathrm{~d}\left(\mathrm{Y}_{\mathrm{N}}\right)$ & $\mathrm{d}\left(\mathrm{Y}_{\mathrm{P}}\right)$ & $\mathrm{d}\left(\mathrm{Y}_{\mathrm{K}}\right)$ \\
\hline & & & & & & & & tha $a^{-1}$ & & & & & t ha & \\
\hline 000 & -65 & 148 & 60 & 28 & -105 & -66 & 79 & 20,00 & $\mathrm{~V}^{+}$ & $\mathrm{V}^{-}$ & $\mathrm{V}^{-}$ & 6,53 & 0,42 & 1,25 \\
\hline 001 & -61 & 130 & 0 & 59 & -75 & -52 & 63 & 18,75 & $\mathrm{~V}^{+}$ & $\mathrm{V}^{-}$ & $\mathrm{V}^{-}$ & 6,27 & 0,17 & 0,17 \\
\hline 002 & -60 & 100 & 0 & 60 & -61 & -39 & 53 & 18,58 & $\mathrm{~V}^{+}$ & $\mathrm{F}^{-}$ & $\mathrm{F}^{-}$ & 6,11 & $-0,17$ & $-0,92$ \\
\hline 003 & -63 & 70 & 0 & 55 & -23 & -39 & 42 & 19,50 & $\mathrm{~V}^{+}$ & $\mathrm{F}^{-}$ & - & 5,86 & $-0,42$ & 0,00 \\
\hline 010 & -42 & 136 & 0 & 21 & -57 & -58 & 52 & 19,58 & $\mathrm{~V}^{+}$ & $F^{*}$ & $\mathrm{~V}^{-}$ & 6,20 & $-0,26$ & 1,00 \\
\hline 011 & -77 & 186 & -29 & 59 & .74 & -66 & 82 & 18,58 & $\mathrm{~V}^{+}$ & $F^{-}$ & $\mathrm{V}^{+}$ & 5,94 & $-0,59$ & 0,17 \\
\hline 012 & -75 & 131 & 0 & 66 & -69 & -52 & 66 & 18,75 & $\mathrm{~V}^{+}$ & $\mathrm{F}^{-}$ & $F^{-}$ & 5,69 & $-0,83$ & $-1,17$ \\
\hline 013 & -68 & 71 & 0 & 61 & -25 & -39 & 44 & 19,92 & $\mathrm{~V}^{+}$ & $F^{-}$ & - & 5,52 & $-1,17$ & 0,00 \\
\hline 020 & -85 & 150 & 28 & 0 & -32 & -63 & 60 & 19,84 & $\mathrm{~V}^{+}$ & $\mathrm{F}^{-}$ & $\mathrm{V}^{-}$ & 5,85 & $-1,00$ & 0,67 \\
\hline 021 & -83 & 145 & 0 & 53 & -58 & -58 & 66 & 19,17 & $\mathrm{~V}^{+}$ & $F^{-}$ & $\mathrm{F}^{-}$ & 5,52 & $-1,25$ & $-0,41$ \\
\hline 022 & -104 & 89 & 0 & 60 & -24 & -21 & 50 & 19,58 & $\mathrm{~V}^{+}$ & $F^{-}$ & $\mathrm{F}^{-}$ & 5,28 & $-1,51$ & $-1,51$ \\
\hline 023 & -142 & 89 & 36 & 59 & -21 & -21 & 61 & 21,09 & $\mathrm{~V}^{+}$ & $F^{-}$ & - & 5,02 & $-1,76$ & 0,00 \\
\hline 030 & -137 & 144 & 41 & -27 & 0 & -22 & 62 & 20,84 & $\mathrm{~V}^{+}$ & - & $\mathrm{V}^{-}$ & 5,44 & 0,00 & 0,42 \\
\hline 031 & -168 & 162 & 37 & 31 & -32 & -30 & 77 & 20,42 & $\mathrm{~V}^{+}$ & - & $F^{-}$ & 5,19 & 0,00 & $-0,67$ \\
\hline 032 & -167 & 117 & 43 & 36 & 0 & -29 & 65 & 21,09 & $\mathrm{~V}^{+}$ & - & $\mathrm{F}^{-}$ & 4,94 & 0,00 & $-1,76$ \\
\hline 033 & -184 & 116 & 88 & 38 & 0 & -58 & 81 & 22,85 & $\mathrm{~V}^{+}$ & - & - & 4,68 & 0,00 & 0,00 \\
\hline 100 & 0 & 85 & 56 & 28 & -89 & -79 & 56 & 26,53 & $\mathrm{~F}^{-}$ & $\mathrm{V}^{-}$ & $\mathrm{V}^{-}$ & $-3,93$ & 0,75 & 1,51 \\
\hline 101 & 0 & 98 & 0 & 61 & -67 & -92 & 53. & 25,02 & $\mathrm{~F}^{-}$ & $\mathbf{V}^{-}$ & $\mathrm{V}^{-}$ & $-3,69$ & 0,50 & 0,33 \\
\hline 102 & 0 & 94 & 0 & 57 & -59 & -92 & 50 & 24,69 & $\mathrm{~F}^{-}$ & $\mathrm{V}^{-}$ & $F^{-}$ & $-3,43$ & 0,25 & $-0,67$ \\
\hline 103 & 0 & 48 & 0 & 28 & 0 & -76 & 25 & 25,36 & $\mathrm{~F}^{-}$ & $F^{-}$ & - & $-3,18$ & $-0,08$ & 0,00 \\
\hline 110 & 0 & 55 & 0 & 22 & -22 & -55 & 26 & 25,78 & $\mathrm{~F}^{-}$ & $\mathrm{V}^{-}$ & $\mathrm{V}^{-}$ & $-3,51$ & 0,09 & 1,26 \\
\hline 111 & 0 & 85 & 0 & 57 & -61 & -81 & 47 & 24,52 & $\mathrm{~F}^{-}$ & $\mathrm{F}^{-}$ & $\mathrm{V}^{-}$ & $-3,27$ & $-0,17$ & 0,08 \\
\hline 112 & -50 & 119 & 0 & 88 & -62 & -95 & 69 & 24,44 & $\mathrm{~V}^{+}$ & $\mathrm{F}^{-}$ & $F^{-}$ & 3,01 & $-0,42$ & $-1,00$ \\
\hline 113 & -24 & 55 & 0 & 74 & -23 & -81 & 43 & 25,44 & $\mathrm{~V}^{+}$ & $F^{-}$ & - & 2,76 & $-0,67$ & 0,00 \\
\hline 120 & 0 & 47 & 0 & 0 & 0 & -47 & 16 & 25,69 & $\mathrm{~F}^{-}$ & $\mathrm{F}^{-}$ & $\mathrm{V}^{-}$ & $-3,10$ & $-0,59$ & 1,00 \\
\hline 121 & -51 & 80 & 0 & 76 & -23 & -82 & 52 & 24,69 & $\mathrm{~V}^{+}$ & $\mathrm{F}^{-}$ & $\mathrm{F}^{-}$ & 2,93 & $-0,92$ & $-0,17$ \\
\hline 122 & -54 & 81 & 0 & 82 & -27 & -82 & 54 & 24,86 & $\mathrm{~V}^{+}$ & $\mathrm{F}^{-}$ & $\mathrm{F}^{-}$ & 2,67 & $-1,17$ & $-1,25$ \\
\hline 123 & -52 & 80 & 0 & 78 & -24 & -82 & 53 & 26,11 & $\mathrm{~V}^{+}$ & $F^{-}$ & - & 2,43 & $-1,42$ & 0,00 \\
\hline 130 & -36 & 96 & 0 & 0 & 0 & -60 & 32 & 26,28 & $\mathrm{~V}^{+}$ & - & $\mathrm{V}^{-}$ & 2,76 & 0,00 & 0,67 \\
\hline 131 & -66 & 99 & 0 & 27 & 0 & -60 & 42 & 25,61 & $\mathrm{~V}^{+}$ & - & $\mathrm{F}^{-}$ & 2,51 & 0,00 & $-0,42$ \\
\hline 132 & -69 & 109 & 0 & 29 & 0 & -69 & 46 & 26,03 & $\mathrm{~V}^{+}$ & - & $F^{-}$ & 2,34 & 0,00 & $-1,50$ \\
\hline 133 & -107 & 107 & 78 & 54 & & 132 & 80 & 27,53 & $\mathrm{~V}^{+}$ & - & - & 2,10 & 0,00 & 0,00 \\
\hline
\end{tabular}


Apêndice 28. Índices DRIS, calculados pelo procedimento proposto por Elwali \& Gascho (1984), diagnose para a correção de $\mathrm{N}, \mathrm{P}$ e $\mathrm{K}$, resposta líquida da produtividade, diagnose para o nutriente deficiente e a resposta da produtividade, para os dados, dos tratamentos com as doses 2 e 3 de $\mathrm{N}$ em todas as combinações de doses de $\mathrm{P}$ e $\mathrm{K}$, do ensaio de adubação $\mathrm{N}, \mathrm{P}$ e $\mathrm{K}$ conduzido em Monte Azul /SP.

\begin{tabular}{|c|c|c|c|c|c|c|c|c|c|c|c|c|c|c|}
\hline \multirow[b]{2}{*}{ Trat. } & \multicolumn{6}{|c|}{ Índices DRIS } & \multirow[b]{2}{*}{$\mathrm{BN}$} & \multirow[b]{2}{*}{ Prod. } & \multicolumn{3}{|c|}{ Diagnose } & \multicolumn{3}{|c|}{ Resposta } \\
\hline & $\overline{\mathrm{N}}$ & $P$ & $\mathrm{~K}$ & $\overline{\mathrm{Ca}}$ & $\mathrm{Mg}$ & $\bar{S}$ & & & $\mathrm{~N}$ & $\mathbf{P}$ & $\bar{K}$ & $d\left(Y_{N}\right)$ & $d\left(Y_{P}\right)$ & $\mathrm{d}\left(\mathrm{Y}_{\mathrm{K}}\right)$ \\
\hline & & & & & & & & $\mathrm{tha}^{-1}$ & & & & & $-\mathrm{tha}^{-1}$ & \\
\hline 200 & 0 & 48 & 28 & 55 & -25 & -106 & 44 & 30,46 & $\mathrm{~F}^{-}$ & $\mathrm{V}^{*}$ & $\mathrm{~V}^{-}$ & $-1,26$ & 1,17 & 1,75 \\
\hline 201 & 0 & 48 & 0 & 57 & -28 & -77 & 35 & 28,71 & $\mathrm{~F}^{-}$ & $\mathrm{V}^{-}$ & $\mathrm{V}^{-}$ & $-1,00$ & 0,92 & 0,59 \\
\hline 202 & 0 & 48 & 0 & 51 & -23 & -76 & 33 & 28,12 & $\mathrm{~F}^{-}$ & $\mathrm{V}^{-}$ & $F^{-}$ & $-0,75$ & 0,67 & $-0,42$ \\
\hline 203 & 0 & 48 & 0 & 0 & 0 & -48 & 16 & 28,54 & $\mathrm{~F}^{-}$ & $\mathrm{V}^{-}$ & - & $-0,58$ & 0,34 & 0,00 \\
\hline 210 & 0 & 33 & 0 & 57 & -24 & -66 & 30 & 29,29 & $\mathrm{~F}^{-}$ & $\mathrm{V}^{-}$ & $\mathrm{V}^{-}$ & $-0,84$ & 0,50 & 1,50 \\
\hline 211 & 0 & 48 & 0 & 60 & -28 & -81 & 36 & 27,79 & $F^{-}$ & $\mathrm{V}^{-}$ & $\mathrm{V}^{-}$ & $-0,67$ & 0,17 & 0,34 \\
\hline 212 & 0 & 48 & 0 & 58 & -27 & -80 & 36 & 27,45 & $F^{-}$ & $\mathrm{F}^{-}$ & $\mathrm{F}^{-}$ & $-0,42$ & $-0,08$ & $-0,75$ \\
\hline 213 & 0 & 48 & 0 & 30 & 0 & -78 & 26 & 28,20 & $\mathrm{~F}^{-}$ & $\mathrm{F}^{-}$ & - & $-0,17$ & $-0,34$ & 0,00 \\
\hline 220 & 0 & 40 & 0 & 29 & 0 & -69 & 23 & 28,79 & $\mathrm{~F}^{-}$ & $F^{-}$ & $\mathrm{V}^{-}$ & $-0,50$ & $-0,25$ & 1,17 \\
\hline 221 & -27 & 48 & 0 & 85 & -25 & -82 & 45 & 27,62 & $\mathrm{~V}^{+}$ & $F^{-}$ & $\mathrm{V}^{-}$ & 0,25 & $-0,50$ & 0,09 \\
\hline 222 & -26 & 48 & 0 & 83 & -24 & -81 & 44 & 27,53 & $\mathrm{~F}^{+}$ & $\mathbf{F}^{-}$ & $F^{-}$ & 0,00 & $-0,84$ & $-1,01$ \\
\hline 223 & 0 & 48 & 26 & 32 & & -106 & 35 & 28,54 & $\mathrm{~V}^{-}$ & $\mathrm{F}^{-}$ & - & 0,17 & $-1,09$ & 0,00 \\
\hline 230 & -27 & 40 & 0 & 55 & 0 & -68 & 32 & 29,04 & $\mathrm{~V}^{+}$ & - & $\mathrm{V}^{-}$ & 0,08 & 0,00 & 0,92 \\
\hline 231 & -29 & 40 & 0 & 57 & 0 & -68 & 32 & 28,12 & $\mathrm{~F}^{+}$ & - & $F^{*}$ & $-0,08$ & 0,00 & $-0,25$ \\
\hline 232 & -27 & 48 & 0 & 60 & 0 & -81 & 36 & 28 , & $\mathrm{F}^{+}$ & - & $F^{-}$ & $-0,42$ &, 00 & $-1,26$ \\
\hline 233 & -30 & 55 & 60 & 27 & & -112 & 47 & 29,63 & $\mathrm{~F}^{+}$ & - & - & $-0,59$ & 0,00 & 0,00 \\
\hline 300 & 0 & -22 & 0 & 22 & 0 & 0 & 7 & 31,72 & - & $\mathrm{F}^{+}$ & $\mathrm{V}^{-}$ & 0,00 & $-1,59$ & 2,01 \\
\hline 301 & 0 & 26 & 0 & 0 & 0 & -26 & 9 & 29,71 & - & $\mathrm{V}^{-}$ & $\mathrm{V}^{-}$ & 0,00 & 1,25 & 0,84 \\
\hline 302 & 0 & 40 & 0 & 0 & 0 & -40 & 13 & 28,87 & - & $\mathrm{V}^{-}$ & $\mathrm{F}^{-}$ & 0,00 & 1,00 & $-0,25$ \\
\hline 303 & 0 & 33 & 0 & 0 & 0 & -33 & 11 & 29,12 & - & $\mathrm{V}^{-}$ & - & 0,00 & 0,75 & 0,00 \\
\hline 310 & -29 & -26 & 0 & 102 & -20 & -27 & 34 & 30,13 & - & $\mathrm{F}^{+}$ & $\mathrm{V}^{-}$ & 0,00 & $-0,84$ & 1,67 \\
\hline 311 & -28 & 26 & 0 & 80 & -22 & -56 & 35 & 28,46 & - & $\mathrm{V}^{-}$ & $\mathrm{V}^{-}$ & 0,00 & 0,59 & 0,59 \\
\hline 312 & -25 & 26 & 0 & 52 & 0 & -53 & 26 & 27,87 & - & $\mathrm{V}^{-}$ & $\mathrm{F}^{-}$ & 0,00 & 0,34 & $-0,50$ \\
\hline 313 & 0 & 33 & 0 & 0 & 0 & -33 & 11 & 28,37 & - & $\mathrm{V}^{-}$ & - & 0,00 & 0,00 & 0,00 \\
\hline 320 & -33 & -28 & -34 & 124 & 0 & -29 & 41 & 29,29 & - & $\mathrm{F}^{+}$ & $\mathrm{F}^{+}$ & 0,00 & $-0,17$ & $-1,42$ \\
\hline 321 & -32 & 26 & -37 & 125 & -24 & -58 & 50 & 27,87 & - & $F^{-}$ & $\mathrm{F}^{+}$ & 0,00 & $-0,17$ & $-0,34$ \\
\hline 322 & -29 & 26 & 0 & 81 & -22 & -56 & 36 & 27,53 & - & $\mathrm{F}^{-}$ & $F^{-}$ & 0,00 & $-0,42$ & $-0,84$ \\
\hline 323 & 0 & 33 & 0 & 0 & 0 & -33 & 11 & 28,37 & - & $F^{-}$ & - & 0,00 & $-0,67$ & 0,00 \\
\hline 330 & -36 & -27 & -61 & 132 & 0 & -8 & 44 & 29,12 & - & - & $\mathrm{F}^{+}$ & 0,00 & 0,00 & $-1,08$ \\
\hline 331 & -35 & 0 & -38 & 101 & 0 & -28 & 34 & 28,04 & - & - & $\mathrm{F}^{+}$ & 0,00 & 0,00 & $-0,09$ \\
\hline 332 & -31 & 26 & 0 & 62 & 0 & -56 & 29 & 27,95 & - & - & $\mathrm{F}^{-}$ & 0,00 & 0,00 & $-1,09$ \\
\hline 333 & -24 & 33 & 0 & 24 & 0 & -33 & 19 & 29,04 & - & - & - & 0,00 & 0,00 & 0,00 \\
\hline
\end{tabular}


Apêndice 29. Índices DRIS, calculados pelo procedimento proposto por Elwali \& Gascho (1984), diagnose para a correção de $\mathrm{N}, \mathrm{P}$ e K, resposta líquida da produtividade, diagnose para o nutriente deficiente e a resposta da produtividade, para os dados, dos tratamentos com as doses 0 e 1 de $\mathrm{N}$ em todas as combinações de doses de $\mathrm{P}$ e $\mathrm{K}$, do ensaio de adubação $\mathrm{N}, \mathrm{P}$ e $\mathrm{K}$ conduzido em Olímpia/SP.

\begin{tabular}{|c|c|c|c|c|c|c|c|c|c|c|c|c|c|c|}
\hline \multirow[b]{2}{*}{ Trat. } & \multicolumn{6}{|c|}{ Índices DRIS } & \multirow[b]{2}{*}{$\mathrm{BN}$} & \multirow[b]{2}{*}{ Prod. } & \multicolumn{3}{|c|}{ Diagnose } & \multicolumn{3}{|c|}{ Resposta } \\
\hline & $\overline{\mathrm{N}}$ & $\mathbf{P}$ & $\mathrm{K}$ & $\mathrm{Ca}$ & $\mathrm{Mg}$ & $\bar{S}$ & & & $\mathrm{~N}$ & $\mathrm{P}$ & $\mathrm{K}$ & $\mathrm{d}\left(\mathrm{Y}_{\mathrm{N}}\right)$ & $\mathrm{d}\left(\mathrm{Y}_{\mathrm{P}}\right)$ & $d\left(Y_{K}\right)$ \\
\hline & & & & & & & & $\mathrm{tha}^{-1}$ & & & & & $-t_{h a}^{-1}$ & \\
\hline 000 & 48 & 72 & & -140 & 52 & -32 & 57 & 26,86 & $\mathrm{~V}^{-}$ & $\mathrm{V}^{-}$ & $\mathrm{V}^{-}$ & 0,25 & 0,77 & 0,77 \\
\hline 001 & 33 & 29 & & -112 & 50 & 0 & 37 & 26,09 & $\mathrm{~V}^{-}$ & $\mathrm{V}^{-}$ & $\mathrm{V}^{-}$ & 0,08 & 0,51 & 0,59 \\
\hline 002 & 27 & 26 & -46 & -126 & 76 & 43 & 57 & 25,50 & $\mathrm{~V}^{-}$ & $\mathrm{V}^{-}$ & $\mathrm{F}^{+}$ & 0,00 & 0,25 & $-0,51$ \\
\hline 003 & 0 & 30 & -48 & -140 & 111 & 47 & 63 & 24,99 & $\mathrm{~F}^{-}$ & $\mathrm{V}^{-}$ & - & $-0,17$ & 0,00 & 0,00 \\
\hline 010 & 39 & 63 & & -149 & 53 & -5 & 52 & 26,09 & $\mathrm{~V}^{-}$ & $\mathrm{F}^{-}$ & $\mathrm{V}^{-}$ & 0,84 & $-0,43$ & 0,51 \\
\hline 011 & 7 & 28 & -46 & -134 & 99 & 45 & 60 & 25,58 & $\mathrm{~V}^{-}$ & $\mathrm{F}^{-}$ & $\mathrm{F}^{+}$ & 0,67 & $-0,77$ & $-0,33$ \\
\hline 012 & -3 & 27 & -52 & -134 & 112 & 50 & 63 & 25,25 & $\mathrm{~F}^{+}$ & $\mathrm{F}^{-}$ & $\mathrm{F}^{+}$ & $-0,52$ & -1.02 & -0.26 \\
\hline 013 & -2 & 31 & -45 & -152 & 117 & 50 & 66 & 24,99 & $\mathrm{~F}^{+}$ & $\mathrm{F}^{-}$ & - & $-0,26$ & $-1,36$ & 0,00 \\
\hline 020 & 35 & 31 & & -143 & 55 & 22 & 48 & 26,52 & $\mathrm{~V}^{-}$ & $\mathrm{F}^{-}$ & $\mathrm{V}^{-}$ & 1,36 & $-1,79$ & 0,17 \\
\hline 021 & 5 & 31 & -43 & -134 & 99 & 42 & 59 & 26,35 & $\mathrm{~V}^{-}$ & $\mathrm{F}^{-}$ & $\mathrm{F}^{+}$ & 1,19 & $-2,04$ & $-0,08$ \\
\hline 022 & 0 & 30 & -48 & -144 & 110 & 52 & 64 & 26,27 & $\mathrm{~V}^{-}$ & $\mathrm{F}^{-}$ & $\mathrm{V}^{+}$ & 1,02 & $-2,37$ & 0,08 \\
\hline 023 & 7 & 36 & & -163 & 89 & 30 & 54 & 26,35 & $\mathrm{~V}^{*}$ & $\mathrm{~F}^{-}$ & - & 0,85 & $-2,63$ & 0,00 \\
\hline 030 & 35 & 36 & & -145 & 51 & 23 & 48 & 28,31 & $\mathrm{~V}^{-}$ & - & $\mathrm{F}^{-}$ & 1,88 & 0,00 & $-0,08$ \\
\hline 031 & 30 & 28 & & -135 & 52 & 24 & 45 & 28,39 & $\mathrm{~V}^{-}$ & - & $\mathrm{F}^{-}$ & 1,70 & 0,00 & $-0,25$ \\
\hline 032 & 33 & 28 & & -144 & 55 & 27 & 48 & 28,64 & $\mathrm{~V}^{-}$ & - & $\mathrm{F}^{-}$ & 1,53 & 0,00 & $-0,34$ \\
\hline 033 & 43 & 36 & 25 & -199 & 65 & 31 & 67 & 28,98 & $\mathrm{~V}^{-}$ & - & - & 1,36 & 0,00 & 0,00 \\
\hline 100 & 40 & 57 & & -124 & 52 & -26 & 50 & 26,61 & $F^{-}$ & $\mathrm{V}^{-}$ & $\mathrm{V}^{-}$ & $-0,76$ & 1,36 & 0,60 \\
\hline 101 & 30 & 0 & 0 & -79 & 50 & 0 & 27 & 26,01 & $F^{-}$ & $\mathrm{V}^{*}$ & $\mathrm{~V}^{*}$ & $-1,02$ & 1,10 & 0,51 \\
\hline 102 & 26 & 0 & -23 & -77 & 74 & 0 & 33 & 25,50 & $\mathrm{~F}^{-}$ & $\mathrm{V}^{-}$ & $\mathrm{F}^{+}$ & $-1,19$ & 0,77 & $-0,34$ \\
\hline 103 & 29 & 28 & -21 & -133 & 75 & 22 & 51 & 25,16 & $\mathrm{~F}^{-}$ & $\mathrm{V}^{-}$ & - & $-1,36$ & 0,43 & 0,00 \\
\hline 110 & 32 & 29 & -20 & -136 & 76 & 20 & 52 & 25,25 & $\mathrm{~F}^{-}$ & $\mathrm{V}^{*}$ & $\mathrm{~F}^{+}$ & $-0,25$ & 0,09 & $-0,34$ \\
\hline 111 & 2 & 0 & -25 & -78 & 101 & 0 & 34 & 24,91 & $\mathrm{~F}^{*}$ & $\mathrm{~F}^{-}$ & $\mathrm{F}^{+}$ & $-0,51$ & $-0,25$ & $-0,18$ \\
\hline 112 & 1 & 0 & -47 & -98 & 101 & 43 & 48 & 24,73 & $\mathrm{~F}^{-}$ & $F^{-}$ & $\mathrm{F}^{+}$ & $-0,69$ & $-0,52$ & 0,00 \\
\hline 113 & 7 & 28 & -21 & -137 & 100 & 23 & 53 & 24,73 & $F^{-}$ & $\mathrm{F}^{-}$ & - & $-0,77$ & $-0,77$ & 0,00 \\
\hline 120 & 4 & 0 & -25 & -83 & 104 & 0 & 36 & 25,16 & $\mathrm{~V}^{-}$ & $F^{-}$ & $\mathrm{F}^{+}$ & 0,25 & $-1,27$ & 0,00 \\
\hline 121 & 0 & 0 & -49 & -75 & 101 & 23 & 41 & 25,16 & $\mathrm{~V}^{-}$ & $F$ & $\mathrm{~V}^{+}$ & 0,08 & $-1,53$ & 0,09 \\
\hline 122 & 2 & 0 & -25 & -82 & 105 & 0 & 36 & 25,25 & $\mathrm{~F}^{-}$ & $\mathrm{F}^{*}$ & $\mathrm{~V}^{+}$ & $-0,17$ & $-1,86$ & 0,25 \\
\hline 123 & 35 & 32 & & -149 & 59 & 23 & 50 & 25,50 & $\mathrm{~F}^{-}$ & $\mathrm{F}^{-}$ & - & $-0,34$ & $-2,12$ & 0,00 \\
\hline 130 & 27 & 0 & -24 & -79 & 76 & 0 & 34 & 26,43 & $\mathrm{~V}^{-}$ & - & $\mathrm{V}^{+}$ & 0,76 & 0,00 & 0,26 \\
\hline 131 & 6 & 0 & -46 & -78 & 98 & 20 & 41 & 26,69 & $\mathrm{~V}^{-}$ & - & $\mathrm{V}^{+}$ & 0,60 & 0,00 & 0,42 \\
\hline 132 & 31 & 0 & -22 & -85 & 75 & 0 & 36 & 27,11 & $\mathrm{~V}^{-}$ & - & $\mathrm{V}^{+}$ & 0,42 & 0,00 & 0,51 \\
\hline 133 & 45 & 30 & & -154 & 58 & 22 & 52 & 27,62 & $\mathrm{~V}^{-}$ & - & - & 0,17 & 0,00 & 0,00 \\
\hline
\end{tabular}


Apêndice 30. Índices DRIS, calculados pelo procedimento proposto por Elwali \& Gascho (1984), diagnose para a correção de $\mathrm{N}, \mathrm{P}$ e $\mathrm{K}$, resposta líquida da produtividade, diagnose para o nutriente deficiente e a resposta da produtividade, para os dados, dos tratamentos com as doses 2 e 3 de $\mathrm{N}$ em todas as combinações de doses de $\mathrm{P}$ e K, do ensaio de adubação N, P e $\mathrm{K}$ conduzido em Olímpia/SP.

\begin{tabular}{|c|c|c|c|c|c|c|c|c|c|c|c|c|c|c|}
\hline \multirow[b]{2}{*}{ Trat. } & \multicolumn{6}{|c|}{ Índices DRIS } & \multirow[b]{2}{*}{$\mathrm{BN}$} & \multirow[b]{2}{*}{ Prod. } & \multicolumn{3}{|c|}{ Diagnose } & \multicolumn{3}{|c|}{ Resposta } \\
\hline & $\overline{\mathrm{N}}$ & $\mathbf{P}$ & $\mathrm{K}$ & $\mathrm{Ca}$ & $\mathrm{Mg}$ & $\bar{S}$ & & & $N$ & $\mathrm{P}$ & $\mathrm{K}$ & $\mathrm{d}\left(\mathrm{Y}_{\mathrm{N}}\right)$ & $\mathrm{d}\left(\mathrm{Y}_{\mathrm{P}}\right)$ & $\mathrm{d}\left(\mathrm{Y}_{\mathrm{K}}\right)$ \\
\hline & & & & & & & & $t_{h a^{-1}}$ & & & & & $-t h a^{-1}$ & \\
\hline 200 & 37 & 30 & & 123 & 56 & 0 & 41 & 27,37 & $\mathrm{~F}^{-}$ & $\mathrm{V}^{-}$ & $\mathrm{V}^{-}$ & $-1,96$ & 1,87 & 0,34 \\
\hline 201 & 30 & 0 & -23 & -83 & 76 & 0 & 35 & 27,03 & $\mathrm{~F}^{-}$ & $\mathrm{V}^{-}$ & $\mathrm{F}^{+}$ & $-2,13$ & 1,61 & $-0,34$ \\
\hline 202 & 30 & 0 & -46 & -80 & 73 & 22 & 42 & 26,69 & $\mathrm{~F}^{-}$ & $\mathrm{V}^{-}$ & $\mathrm{F}^{+}$ & $-2,29$ & 1,27 & $-0,17$ \\
\hline 203 & 36 & 31 & & 142 & 52 & 22 & 47 & 26,52 & $\mathrm{~F}^{-}$ & $\mathrm{V}^{*}$ & - & $-2,55$ & 1,02 & 0,00 \\
\hline 210 & 5 & 27 & $-26-$ & .114 & 108 & 0 & 47 & 25,50 & $\mathrm{~F}^{-}$ & $\mathrm{V}^{-}$ & $\mathrm{F}^{+}$ & $-1,44$ & 0,59 & $-0,08$ \\
\hline 211 & 0 & 0 & -55 & -80 & 110 & 24 & 45 & 25,42 & $\mathrm{~F}^{-}$ & $\mathrm{V}^{-}$ & $\mathrm{F}^{+}$ & $-1,61$ & 0,34 & 0,00 \\
\hline 212 & 27 & 0 & -73 & -81 & 104 & 24 & 52 & 25,42 & $\mathrm{~F}^{-}$ & $\mathrm{V}^{-}$ & $\mathrm{V}^{+}$ & $-1,78$ & 0,00 & 0,08 \\
\hline 213 & 36 & 0 & $-21-$ & -112 & 75 & 21 & 44 & 25,50 & $\mathrm{~F}^{-}$ & $F^{-}$ & - & $-1,95$ & $-0,34$ & 0,00 \\
\hline 220 & -3 & -20 & -60 & -81 & 138 & 27 & 55 & 24,91 & $\mathrm{~V}^{+}$ & $\mathrm{V}^{+}$ & $\mathrm{V}^{+}$ & ,93 & 0,76 & 0,17 \\
\hline 221 & 23 & -20 & -85 & -77 & 130 & 30 & 61 & 25,08 & $\mathrm{~F}^{-}$ & $\mathrm{V}^{+}$ & $\mathrm{V}^{+}$ & $-1,10$ & 1,01 & 0,34 \\
\hline 222 & 51 & 0 & -80 & -81 & 82 & 28 & 54 & 25,42 & $\mathrm{~F}^{-}$ & $F^{-}$ & $\mathrm{V}^{+}$ & $-1,19$ & $-1,27$ & 0,42 \\
\hline 223 & 41 & 0 & $-21-$ & -119 & 79 & 21 & 47 & 25,84 & $\mathrm{~F}^{-}$ & $\mathrm{F}^{-}$ & - & $-1,45$ & $-1,61$ & 0,00 \\
\hline 230 & 26 & 0 & -94 & -78 & 117 & 29 & 57 & 25,67 & $F^{-}$ & - & $\mathrm{V}^{+}$ & $-0,34$ & 0,00 & 0,42 \\
\hline 231 & 35 & 0 & -94 & -77 & 106 & 30 & 57 & 26,09 & $F^{-}$ & - & $\mathrm{V}^{+}$ & $-0,60$ & 0,00 & 0,60 \\
\hline 232 & 63 & 0 & -56 & -87 & 81 & 0 & 48 & 26,69 & $F^{-}$ & - & $\mathrm{V}^{+}$ & $-0,76$ & 0,00 & 0,76 \\
\hline 233 & 100 & 57 & $-21-$ & -138 & 55 & -53 & 71 & 27,45 & $\mathrm{~F}^{-}$ & - & - & $-0,86$ & 0,00 & 0,00 \\
\hline 300 & 39 & 0 & $-24-$ & -124 & 86 & 24 & 50 & 29,33 & - & $\mathrm{V}^{-}$ & $\mathrm{F}^{+}$ & 0,00 & 2,39 & $-0,17$ \\
\hline 301 & 60 & 0 & $-84-$ & -114 & 84 & 54 & 66 & 29,16 & - & $\mathrm{V}^{-}$ & $\mathrm{F}^{+}$ & 0,00 & 2,13 & $-0,18$ \\
\hline 302 & 69 & 0 & -89 & -112 & 80 & 52 & 67 & 28,98 & - & $\mathrm{V}^{-}$ & $\mathrm{V}^{+}$ & 0,00 & 1,78 & 0,09 \\
\hline 303 & 80 & 32 & -76 & 157 & 75 & 46 & 78 & 29,07 & - & $\mathrm{V}^{*}$ & - & 0,00 & 1,62 & 0,00 \\
\hline 310 & 26 & -22 & $-98-$ & -117 & 153 & 58 & 79 & 26,94 & - & $\mathrm{F}^{+}$ & $\mathrm{V}^{+}$ & 0,00 & $-1,10$ & 0,09 \\
\hline 311 & 38 & $-20-$ & $-110-$ & -106 & 139 & 59 & 79 & 27,03 & - & $\mathrm{F}^{+}$ & $\mathrm{V}^{+}$ & 0,00 & $-0,85$ & 0,17 \\
\hline 312 & 69 & & -103 & -89 & 90 & 34 & 64 & 27,20 & - & $\mathrm{V}^{-}$ & $\mathrm{V}^{+}$ & 0,00 & 0,59 & 0,25 \\
\hline 313 & 82 & 30 & $-83-$ & -155 & 80 & 45 & 79 & 27,45 & - & $\mathrm{V}^{-}$ & - & 0,00 & 0,16 & 0,00 \\
\hline 320 & 29 & & -155 & -87 & 163 & 44 & 81 & 25,84 & - & $\mathrm{F}^{-}$ & $\mathrm{V}^{+}$ & 0,00 & $-0,17$ & 0,34 \\
\hline 321 & 44 & & -159 & -83 & 147 & 44 & 81 & 26,18 & - & $\mathrm{F}^{-}$ & $\mathrm{V}^{+}$ & 0,00 & $-0,51$ & 0,43 \\
\hline 322 & 78 & & -117 & -91 & 95 & 36 & 70 & 26,61 & - & $\mathrm{F}^{-}$ & $\mathrm{V}^{+}$ & 0,00 & $-0,84$ & 0,68 \\
\hline 323 & 133 & -21 & $-87-$ & -109 & 83 & 1 & 72 & 27,29 & - & $\mathrm{V}^{+}$ & - & 0,00 & 1,02 & 0,00 \\
\hline 330 & 47 & & -196 & -82 & 170 & 53 & 93 & 26,01 & - & - & $\mathrm{V}^{+}$ & 0,00 & 0,00 & 0,68 \\
\hline 331 & 84 & & -184 & -83 & 129 & 48 & 89 & 26,69 & - & - & $\mathrm{V}^{+}$ & 0,00 & 0,00 & 0,76 \\
\hline 332 & 94 & 0 & -126 & -92 & 89 & 35 & 73 & 27,45 & - & - & $\mathrm{V}^{+}$ & 0,00 & 0,00 & 0,86 \\
\hline 333 & 179 & 30 & $-69-$ & 145 & 75 & -70 & 95 & 28,31 & - & - & - & 0,00 & 0,00 & 0,00 \\
\hline
\end{tabular}


Apêndice 31. Índices DRIS, calculados pelo procedimento proposto por Elwali \& Gascho (1984), diagnose para a correção de $\mathrm{N}, \mathrm{P}$ e $\mathrm{K}$, resposta líquida da produtividade, diagnose para o nutriente deficiente e a resposta da produtividade, para os dados, dos tratamentos com as doses 0 e 1 de $\mathrm{N}$ em todas as combinações de doses de $\mathrm{P}$ e $\mathrm{K}$, do ensaio de adubação $\mathrm{N}, \mathrm{P}$ e $\mathrm{K}$ conduzido em Pirassununga/SP.

\begin{tabular}{|c|c|c|c|c|c|c|c|c|c|c|c|c|c|c|}
\hline \multirow[b]{2}{*}{ Trat. } & \multicolumn{6}{|c|}{ Índices DRIS } & \multirow[b]{2}{*}{$\mathrm{BN}$} & \multirow[b]{2}{*}{ Prod. } & \multicolumn{3}{|c|}{ Diagnose } & \multicolumn{3}{|c|}{ Resposta } \\
\hline & $\overline{\mathrm{N}}$ & $P$ & $\mathrm{~K}$ & $\mathrm{Ca}$ & $\mathrm{Mg}$ & $\bar{S}$ & & & $\mathrm{~N}$ & $\mathrm{P}$ & $\overline{\mathrm{K}}$ & $\overline{d\left(Y_{N}\right)}$ & $d\left(Y_{P}\right)$ & $\overline{d\left(Y_{K}\right)}$ \\
\hline & & & & & & & & $\mathrm{tha}^{-1}$ & & & & & tha- & \\
\hline 000 & -83 & 26 & -72 & 116 & 72 & -60 & 72 & 35,50 & $\mathrm{~V}^{+}$ & $\mathrm{V}^{-}$ & $\mathrm{V}^{+}$ & 1,76 & 2,18 & 0,95 \\
\hline 001 & -68 & 33 & 0 & 75 & 29 & -69 & 46 & & $\mathrm{~V}^{+}$ & $v^{-}$ & $\mathrm{V}^{-}$ & 1,49 & 1,23 & 0,55 \\
\hline 002 & -60 & 33 & 0 & 72 & 23 & -68 & 43 & 35,90 & $\mathrm{~V}^{+}$ & $\mathrm{V}^{-}$ & $\mathrm{V}^{-}$ & 1,36 & 0,27 & 1,90 \\
\hline 003 & -71 & 48 & 63 & 72 & & -112 & 61 & 34,00 & $\mathrm{~V}^{+}$ & $\mathrm{F}^{-}$ & - & 1,09 & $-0,82$ & 0,00 \\
\hline 010 & -73 & 33 & -69 & 120 & 61 & -72 & 71 & 33,32 & $\mathrm{~V}^{+}$ & $\mathrm{V}^{-}$ & $\mathrm{V}^{+}$ & 2,58 & 3,13 & 1,90 \\
\hline 011 & -66 & 33 & 0 & 76 & 27 & -70 & 45 & 35,22 & $\mathrm{~V}^{+}$ & $\mathrm{V}^{-}$ & $\mathrm{F}^{-}$ & 2,45 & 2,17 & $-0,41$ \\
\hline 012 & -87 & 33 & 26 & 74 & 22 & -68 & 52 & 35,63 & $\mathrm{~V}^{+}$ & $\mathrm{V}^{-}$ & $\mathrm{V}^{-}$ & 2,31 & 0,95 & 0,81 \\
\hline 013 & -106 & 75 & 66 & 78 & & -113 & 73 & 34,82 & $\mathrm{~V}^{+}$ & $\mathrm{V}^{-}$ & - & 2,04 & 0,00 & 0,00 \\
\hline 020 & -57 & 77 & -93 & 106 & 52 & -85 & 78 & 30,19 & $\mathrm{~V}^{+}$ & $\mathrm{V}^{-}$ & $\mathrm{V}^{+}$ & 3,54 & 3,94 & 2,86 \\
\hline 021 & -56 & 33 & 0 & 66 & 24 & -67 & 41 & 33,05 & $\mathrm{~V}^{+}$ & $\mathrm{V}^{-}$ & $\mathrm{F}^{-}$ & 3,40 & 2,86 & $-1,63$ \\
\hline 022 & -54 & 33 & 0 & 67 & 20 & -66 & 40 & 34,68 & $\mathrm{~V}^{+}$ & $\mathrm{V}^{-}$ & $\mathrm{F}^{-}$ & 3,13 & 1,90 & $-0,14$ \\
\hline 023 & -123 & 74 & 68 & 72 & $20-$ & -112 & 78 & 34,82 & $\mathrm{~V}^{+}$ & $\mathrm{V}^{-}$ & - & 2,99 & 0,96 & 0,00 \\
\hline 030 & 22 & 99 - & -118 & 63 & 29 & -95 & 71 & 26,25 & $\mathrm{~F}^{-}$ & - & $\mathrm{V}^{+}$ & $-4,35$ & 0,00 & 3,94 \\
\hline 031 & -20 & 48 & 0 & 29 & 20 & -77 & 32 & 30,19 & $\mathrm{~V}^{+}$ & - & $\mathrm{F}^{-}$ & 4,22 & 0,00 & $-2,59$ \\
\hline 032 & -21 & 48 & 0 & 28 & 21 & -76 & 32 & & $\mathrm{~V}^{+}$ & - & $\mathrm{F}^{-}$ & 4,08 & 0,00 & $-1,08$ \\
\hline 033 & -119 & 95 & 67 & 57 & $25-$ & -124 & 81 & & $\mathrm{~V}^{+}$ & - & - & 5 & 0 & 0,00 \\
\hline 100 & -63 & $24-$ & -224 & 186 & 168 & -90 & 126 & 37,26 & $\mathrm{~V}^{+}$ & $\mathrm{V}^{-}$ & $\mathrm{V}^{+}$ & 0,28 & 1,36 & 0,68 \\
\hline 101 & -79 & -57 & -78 & 154 & 137 & -77 & 97 & 37,94 & $\mathrm{~V}^{+}$ & $\mathrm{F}^{+}$ & $\mathrm{F}^{+}$ & 0,14 & $-0,27$ & $-0,68$ \\
\hline 102 & -63 & 33 & -21 & 74 & 49 & -72 & 52 & 37,26 & $\mathrm{~F}^{+}$ & $\mathrm{F}^{-}$ & $\mathrm{F}^{+}$ & 0,00 & $-0,68$ & $-2,17$ \\
\hline 103 & -53 & 57 & 0 & 74 & 21 & -99 & 51 & 35,09 & $\mathrm{~F}^{+}$ & $\mathrm{F}^{-}$ & - & $-0,14$ & $-1,77$ & 0,00 \\
\hline 110 & -56 & $-33-$ & 209 & 196 & 156 & -53 & 117 & 35,90 & $\mathrm{~V}^{+}$ & $\mathrm{F}^{+}$ & $\mathrm{V}^{+}$ & 1,23 & $-2,17$ & 1,77 \\
\hline 111 & -75 & -53 & -72 & 155 & 118 & -74 & 91 & 37 , & $\mathrm{V}^{+}$ & $\mathrm{F}^{+}$ & $\mathrm{V}^{+}$ & 1,09 & $-1,22$ & 0,27 \\
\hline 112 & -69 & -49 & -20 & 106 & 71 & -39 & 59 & 37,94 & $\mathrm{~V}^{+}$ & $\mathrm{F}^{+}$ & $\mathrm{F}^{+}$ & 0,96 & $-0,13$ & $-1,08$ \\
\hline 113 & -62 & 41 & 0 & 81 & 24 & -84 & 49 & 36,86 & $\mathrm{~V}^{+}$ & $\mathrm{F}^{-}$ & - & 0,81 & $-0,95$ & 0,00 \\
\hline 120 & -36 & $57-$ & -214 & 178 & 121 - & -106 & 119 & 33,73 & $\mathrm{~V}^{+}$ & $\mathrm{V}^{-}$ & $\mathrm{V}^{+}$ & 2,17 & 3,13 & 2,72 \\
\hline 121 & -67 & -51 & -68 & 143 & 115 & -72 & 86 & 36,45 & $\mathrm{~V}^{+}$ & $\mathrm{F}^{+}$ & $\mathrm{V}^{+}$ & 2,04 & $-2,04$ & 1,36 \\
\hline 122 & -64 & -47 & 0 & 99 & 50 & -37 & 50 & 37,81 & $\mathrm{~V}^{+}$ & $\mathrm{F}^{+}$ & $\mathrm{V}^{-}$ & 1,77 & $-0,95$ & 0,00 \\
\hline 123 & -63 & 41 & 0 & 78 & & -114 & 59 & 37,81 & $\mathrm{~V}^{+}$ & $\mathrm{V}^{-}$ & - & 1,63 & 0,00 & 0,00 \\
\hline 130 & 18 & 104. & 224 & 129 & $120-1$ & -147 & 124 & 30,60 & $\mathrm{~F}^{*}$ & - & $\mathrm{V}^{+}$ & $-3,13$ & 0,00 & 3,81 \\
\hline 131 & -52 & 33 & -65 & 93 & $93-1$ & -103 & 73 & 34,41 & $\mathrm{~V}^{+}$ & - & $\mathrm{V}^{+}$ & 2,85 & 0,00 & 2,45 \\
\hline 132 & -54 & 33 & -22 & 58 & $85-1$ & -100 & 59 & 36,86 & $\mathrm{~V}^{+}$ & - & $\mathrm{V}^{+}$ & 2,72 & 0,00 & 0,95 \\
\hline 133 & -59 & 48 & 0 & 59 & $66-1$ & -114 & 58 & 37,81 & $\mathrm{~V}^{+}$ & - & - & 2,58 & 0,00 & 0,00 \\
\hline
\end{tabular}


Apêndice 32. Índices DRIS, calculados pelo procedimento proposto por Elwali \& Gascho (1984), diagnose para a correção de $\mathrm{N}, \mathrm{P}$ e $\mathrm{K}$, resposta líquida da produtividade, diagnose para o nutriente deficiente e a resposta da produtividade, para os dados, dos tratamentos com as doses 2 e 3 de $\mathrm{N}$ em todas as combinações de doses de $\mathrm{P}$ e $\mathrm{K}$, do ensaio de adubação $\mathrm{N}, \mathrm{P}$ e $\mathrm{K}$ conduzido em Pirassununga /SP.

\begin{tabular}{|c|c|c|c|c|c|c|c|c|c|c|c|c|c|c|}
\hline \multirow[b]{2}{*}{ Trat. } & \multicolumn{6}{|c|}{ Índices DRIS } & \multirow[b]{2}{*}{$\mathrm{BN}$} & \multirow[b]{2}{*}{ Prod. } & \multicolumn{3}{|c|}{ Diagnose } & \multicolumn{3}{|c|}{ Resposta } \\
\hline & $\overline{\mathrm{N}}$ & $P$ & $\bar{K}$ & $\overline{\mathrm{Ca}}$ & $\mathrm{Mg}$ & $\bar{S}$ & & & $\mathrm{~N}$ & $\mathrm{P}$ & $\mathrm{K}$ & $d\left(Y_{N}\right)$ & $d\left(Y_{P}\right)$ & $\mathrm{d}\left(\mathrm{Y}_{\mathrm{K}}\right)$ \\
\hline & & & & & & & & $\mathrm{tha}^{-1}$ & & & & & tha ${ }^{-1}$ & \\
\hline 200 & -51 & 31 & -290 & 206 & 187 & -83 & 141 & 37,54 & $\mathrm{~F}^{+}$ & $\mathrm{V}^{-}$ & $\mathrm{V}^{+}$ & $-0,96$ & 0,41 & 0,54 \\
\hline 201 & -48 & -60 & -150 & 164 & 153 & -60 & 106 & 38,08 & $\mathrm{~F}^{+}$ & $\mathrm{V}^{+}$ & $\mathrm{F}^{+}$ & $-1,22$ & 0,68 & $-0,82$ \\
\hline 202 & -59 & 33 & -72 & 105 & 98 & -105 & 79 & 37,26 & $\mathrm{~F}^{+}$ & $\mathrm{F}^{-}$ & $\mathrm{F}^{+}$ & $-1,36$ & $-1,64$ & $-2,31$ \\
\hline 203 & 1 & 57 & -59 & 71 & 83 & -154 & 71 & 34,95 & $\mathrm{~V}^{-}$ & $\mathrm{F}^{-}$ & - & 1,49 & $-2,72$ & 0,00 \\
\hline 210 & -48 & -28 & -274 & 221 & 176 & -46 & 132 & 37,13 & $\mathrm{~F}^{+}$ & $\mathrm{F}^{+}$ & $\mathrm{V}^{+}$ & 0,00 & $-1,23$ & 1,63 \\
\hline 211 & -55 & -59 & -122 & 170 & 145 & -79 & 105 & 38,76 & $\mathrm{~F}^{+}$ & $\mathrm{F}^{+}$ & $\mathrm{V}^{+}$ & $-0,27$ & $-0,27$ & 0,14 \\
\hline 212 & -64 & -25 & -69 & 145 & 119 & -106 & 88 & 38,90 & $\mathrm{~F}^{+}$ & $\mathrm{V}^{+}$ & $\mathrm{F}^{+}$ & $-0,41$ & 0,68 & $-1,23$ \\
\hline 213 & -55 & 41 & -57 & 101 & & -116 & 76 & 37,67 & $\mathrm{~F}^{+}$ & $\mathrm{F}^{-}$ & - & $-0,68$ & $-1,77$ & 0,00 \\
\hline 220 & -32 & 63 & -269 & 202 & 138 & -102 & 134 & 35 & $\mathrm{~V}^{+}$ & $\mathrm{V}^{-}$ & $\mathrm{V}^{+}$ & 0,82 & 2,17 & 2,59 \\
\hline 221 & -47 & -57 & -119 & 159 & 141 & -78 & 100 & 38,49 & $\mathrm{~V}^{+}$ & $\mathrm{F}^{+}$ & $\mathrm{V}^{+}$ & 0,68 & $-1,23$ & 1,09 \\
\hline 222 & -63 & -51 & -68 & 132 & 123 & -71 & 85 & 39,58 & $\mathrm{~V}^{+}$ & $\mathrm{F}^{+}$ & $\mathrm{F}^{+}$ & 0,54 & 0,00 & $-0,14$ \\
\hline 223 & -61 & 41 & -56 & 97 & 98. & -119 & 79 & 39,44 & $\mathrm{~V}^{+}$ & $\mathrm{F}^{-}$ & - & 0,27 & $-0,95$ & 0,00 \\
\hline 230 & 16 & 112 & -270 & 151 & 136 & -144 & 138 & 33,73 & $F^{-}$ & - & $\mathrm{V}^{+}$ & $-1,77$ & 0,00 & 3,53 \\
\hline 231 & -31 & 12 & -116 & 108 & 136 & -109 & 85 & 37,26 & $\mathrm{~V}^{+}$ & - & $\mathrm{V}^{+}$ & 1,64 & 0,00 & 2,32 \\
\hline 232 & -33 & 12 & -65 & 64 & 128 & -105 & 68 & 39,58 & $\mathrm{~V}^{+}$ & - & $\mathrm{V}^{+}$ & 1,36 & 0,00 & 0,81 \\
\hline 233 & -37 & 10 & -33 & 31 & 134 & -105 & 58 & 40,39 & $\mathrm{~V}^{+}$ & - & - & 1,23 & 0,00 & 0,00 \\
\hline 300 & -50 & 31 & -279 & 196 & 185 & -83 & 137 & 36,58 & - & $\mathrm{F}^{-}$ & $\mathrm{V}^{+}$ & 0,00 & $-0,55$ & 0,28 \\
\hline 301 & -37 & 43 & -186 & 123 & 146 & -88 & 104 & 36,86 & - & $\mathrm{F}^{-}$ & $\mathrm{F}^{+}$ & 0,00 & $-1,63$ & $-0,96$ \\
\hline 302 & -1 & 33 & -100 & 69 & 98 & -100 & 67 & 35,90 & - & $\mathrm{F}^{-}$ & $\mathrm{F}^{+}$ & 0,00 & $-2,59$ & $-2,44$ \\
\hline 303 & 38 & 88 & -124 & 64 & 90. & -156 & 93 & 33,46 & - & $\mathrm{F}^{-}$ & - & 0,00 & $-3,53$ & 0,00 \\
\hline 310 & -47 & 53 & -263 & 201 & 136 & -80 & 130 & 37,13 & - & $\mathrm{V}^{-}$ & $\mathrm{V}^{+}$ & 0,00 & 0,41 & 1,36 \\
\hline 311 & -42 & -54 & -143 & 157 & 135 & -54 & 98 & 38,49 & - & $\mathrm{V}^{+}$ & $\mathrm{F}^{+}$ & 0,00 & 0,68 & 0,00 \\
\hline 312 & -32 & -46 & -92 & 121 & 115 & -66 & 79 & 38,49 & - & $\mathrm{V}^{+}$ & $\mathrm{F}^{+}$ & 0,00 & 1,63 & $-1,50$ \\
\hline 313 & 19 & 41 & -83 & 66 & 93 & -135 & 73 & 36,99 & - & $\mathrm{F}^{-}$ & - & 0,00 & $-2,72$ & 0,00 \\
\hline 320 & -36 & 53 & -253 & 190 & 124 & -78 & 122 & 36,72 & - & $\mathrm{V}^{-}$ & $\mathrm{V}^{+}$ & 0,00 & 1,22 & 2,45 \\
\hline 321 & -40 & -53 & -115 & 149 & 132 & -73 & 94 & 39,17 & - & $\mathrm{F}^{+}$ & $\mathrm{V}^{+}$ & 0,00 & $-0,27$ & 0,95 \\
\hline 322 & -55 & -47 & -69 & 116 & 123 & -67 & 80 & 40,12 & - & $\mathrm{V}^{+}$ & $\mathrm{F}^{+}$ & 0,00 & 0,82 & $-0,41$ \\
\hline 323 & -29 & 33 & -32 & 29 & 94 & -96 & 52 & 39,71 & - & $\mathrm{F}^{-}$ & - & 0,00 & $-1,91$ & 0,00 \\
\hline 330 & 6 & 109 & -247 & 148 & 127 & -142 & 130 & 35,50 & - & - & $\mathrm{V}^{+}$ & 0,00 & 0,00 & 3,40 \\
\hline 331 & -4 & 12 & -114 & 77 & 136 & -107 & 75 & 38,90 & - & - & $\mathrm{V}^{+}$ & 0,00 & 0,00 & 2,04 \\
\hline 332 & -32 & 12 & -64 & 58 & 128 & -101 & 66 & 40,94 & - & - & $\mathrm{V}^{+}$ & 0,00 & 0,00 & 0,68 \\
\hline 333 & -35 & 10 & -36 & -29 & 164 & -74 & 58 & 41,62 & - & - & - & 0,00 & 0,00 & 0,00 \\
\hline
\end{tabular}


Apêndice 33. Índices DRIS, calculados pelo procedimento proposto por Jones (1981), diagnose para a correção de N, P e K, resposta líquida da produtividade, diagnose para o nutriente deficiente e a resposta da produtividade, para os dados, dos tratamentos com as doses 0 e 1 de $\mathrm{N}$ em todas as combinações de doses de $\mathrm{P}$ e $\mathrm{K}$, do ensaio de adubação $\mathrm{N}$, $\mathrm{P}$ e $\mathrm{K}$ conduzido em Araraquara/SP.

\begin{tabular}{|c|c|c|c|c|c|c|c|c|c|c|c|c|c|c|}
\hline \multirow[b]{2}{*}{ Trat. } & \multicolumn{6}{|c|}{ Índices DRIS } & \multirow[b]{2}{*}{$\mathrm{BN}$} & \multirow[b]{2}{*}{ Prod. } & \multicolumn{3}{|c|}{ Diagnose } & \multicolumn{3}{|c|}{ Resposta } \\
\hline & $\mathrm{N}$ & $\mathbf{P}$ & $\mathrm{K}$ & $\mathrm{Ca}$ & $\mathrm{Mg}$ & $S$ & & & $\mathrm{~N}$ & $\mathbf{P}$ & $\mathrm{K}$ & $\mathrm{d}\left(\mathrm{Y}_{\mathrm{N}}\right)$ & $d\left(Y_{P}\right)$ & $d\left(Y_{K}\right)$ \\
\hline & & & & & & & & $\mathrm{tha}^{-1}$ & & & & & $t \mathrm{ha}^{-1}$ & \\
\hline 000 & -14 & 55 & 63 & -71 & -61 & 28 & 49 & 31,33 & $\mathrm{~V}^{+}$ & $\mathrm{V}^{-}$ & $\mathrm{F}^{-}$ & 3,64 & 0,00 & $-2,18$ \\
\hline 001 & -14 & 52 & 84 & -59 & -90 & 27 & 54 & 33,51 & $\mathrm{~V}^{+}$ & $\mathrm{F}^{-}$ & $\mathrm{F}^{-}$ & 3,36 & $-0,44$ & $-1,02$ \\
\hline 002 & -13 & 83 & 100 & $-58-$ & -136 & 23 & 69 & 34,53 & $\mathrm{~V}^{+}$ & $\mathrm{F}^{-}$ & $\mathrm{V}^{*}$ & 2,92 & $-0,88$ & 0,14 \\
\hline 003 & -1 & 85 & 122 & $-59-$ & -183 & 37 & 81 & 34,39 & $\mathrm{~V}^{+}$ & $\mathrm{F}^{-}$ & - & 2,48 & $-1,16$ & 0,00 \\
\hline 010 & -12 & 83 & 58 & -77 & -66 & 15 & 52 & 31,33 & $\mathrm{~V}^{+}$ & $\mathrm{V}^{-}$ & $\mathrm{F}^{-}$ & 3,35 & 0,00 & $-2,62$ \\
\hline 011 & -6 & 87 & 84 & $-59-$ & -104 & -1 & 57 & 33,95 & $\mathrm{~V}^{+}$ & $\mathrm{F}^{-}$ & $\mathrm{F}^{-}$ & 3,06 & $-0,44$ & $-1,46$ \\
\hline 012 & -4 & 87 & 106 & $-58-$ & -133 & 3 & 65 & 35,41 & $\mathrm{~V}^{+}$ & $\mathrm{F}^{-}$ & $\mathrm{F}^{-}$ & 2,62 & $-0,73$ & $-0,14$ \\
\hline 013 & 6 & 89 & 128 & $-60-$ & -179 & 16 & 80 & 35,55 & $\mathrm{~F}^{-}$ & $F^{-}$ & - & $-2,19$ & $-1,17$ & 0,00 \\
\hline 020 & -2 & 87 & 61 & -78 & -64 & -5 & 50 & 31,33 & $\mathrm{~V}^{+}$ & $\mathrm{V}^{-}$ & $\mathrm{F}^{-}$ & 3,06 & 0,15 & $-3,06$ \\
\hline 021 & -8 & 86 & 86 & -68 & -91 & -4 & 57 & 34,39 & $\mathrm{~V}^{+}$ & $\mathrm{F}^{-}$ & $\mathrm{F}^{-}$ & 2,62 & $-0,14$ & $-1,75$ \\
\hline 022 & -3 & 90 & 112 & $-65-$ & -114 & -20 & 67 & 36,14 & $\mathrm{~V}^{+}$ & $F^{-}$ & $F^{-}$ & 2,33 & $-0,58$ & $-0,58$ \\
\hline 023 & 4 & 93 & 137 & $-71-$ & -156 & -7 & 78 & 36,72 & $\mathrm{~F}^{-}$ & $F^{-}$ & - & $-1,89$ & $-1,02$ & 0,00 \\
\hline 030 & -22 & 108 & 54 & -96 & -46 & 2 & 55 & 31,18 & $\mathrm{~V}^{+}$ & - & $F^{-}$ & 2,63 & 0,00 & $-3,35$ \\
\hline 031 & -18 & 84 & 88 & -81 & -65 & -8 & 57 & 34,53 & $\mathrm{~V}^{+}$ & - & $\mathrm{F}^{-}$ & 2,34 & 0,00 & $-2,19$ \\
\hline 032 & -14 & 90 & 119 & -77 & -98 & -20 & 70 & 36,72 & $\mathrm{~V}^{+}$ & - & $\mathrm{F}^{-}$ & 2,04 & 0,00 & $-1,02$ \\
\hline 033 & -8 & 97 & 147 & $-86-$ & -119 & -31 & 81 & 37,74 & $\mathrm{~V}^{+}$ & - & - & 1,60 & 0,00 & 0,00 \\
\hline 100 & 27 & 22 & 62 & -29 & -73 & -10 & 37 & 34,97 & $\mathrm{~F}^{-}$ & $\mathrm{V}^{-}$ & $\mathrm{F}^{-}$ & $-2,33$ & 0,29 & $-1,90$ \\
\hline 101 & 17 & 54 & 75 & -30 & -92 & -24 & 49 & 36,87 & $\mathrm{~F}^{-}$ & $F^{-}$ & $\mathrm{F}^{-}$ & $-1,89$ & $-0,14$ & $-0,58$ \\
\hline 102 & 20 & 52 & 100 & $-31-$ & -120 & -21 & 57 & 37,45 & $\mathrm{~F}^{-}$ & $F^{-}$ & $\mathrm{V}^{-}$ & $-1,46$ & $-0,58$ & 0,58 \\
\hline 103 & 27 & 53 & 124 & $-41-$ & -148 & -15 & 68 & 36,87 & $\mathrm{~F}^{-}$ & $F^{-}$ & - & $-1,16$ & $-0,87$ & 0,00 \\
\hline 110 & 28 & 57 & 51 & -37 & -77 & -22 & 45 & 34,68 & $\mathrm{~F}^{-}$ & $\mathrm{V}^{-}$ & $F^{-}$ & $-2,04$ & 0,29 & $-2,33$ \\
\hline 111 & 26 & 55 & 76 & $-30-$ & -105 & -22 & 52 & 37, & $\mathrm{~F}^{-}$ & $\mathrm{V}^{-}$ & $\mathrm{F}^{-}$ & $-1,46$ & 0,00 & $-1,02$ \\
\hline 112 & 25 & 52 & 100 & $-37-$ & -119 & -22 & 59 & 38,03 & $\mathrm{~F}^{-}$ & $F^{-}$ & $\mathrm{V}^{-}$ & $-1,17$ & $-0,44$ & 0,29 \\
\hline 113 & 36 & 59 & 128 & $-44-$ & -143 & -35 & 74 & 37,74 & $\mathrm{~F}^{-}$ & $F$ & - & $-0,87$ & $-0,87$ & 0,00 \\
\hline 120 & 33 & 57 & 51 & -44 & -76 & -22 & 47 & 34,39 & $\mathrm{~F}^{-}$ & $v^{-}$ & $\mathrm{F}^{-}$ & $-1,60$ & 0,58 & $-2,62$ \\
\hline 121 & 30 & 58 & 82 & -37 & -88 & -45 & 57 & 37,01 & $\mathrm{~F}^{-}$ & $\mathrm{V}^{-}$ & $F^{-}$ & $-1,17$ & 0,14 & $-1,46$ \\
\hline 122 & 29 & 59 & 106 & $-39-$ & -114 & -40 & 65 & 38,47 & $\mathrm{~F}^{-}$ & $\mathrm{F}^{-}$ & $F^{-}$ & $-0,87$ & $-0,29$ & $-0,14$ \\
\hline 123 & 30 & 61 & 133 & $-52-$ & -140 & -32 & 75 & 38,61 & $\mathrm{~F}^{-}$ & $\mathrm{F}^{*}$ & - & $-0,59$ & $-0,73$ & 0,00 \\
\hline 130 & 26 & 57 & 55 & -53 & -63 & -22 & 46 & 33,81 & $\mathrm{~F}^{-}$ & - & $\mathrm{F}^{-}$ & $-1,31$ & 0,00 & $-3,06$ \\
\hline 131 & 22 & 59 & 84 & -49 & -73 & -43 & 55 & 36,87 & $\mathrm{~F}^{-}$ & - & $\mathrm{F}^{-}$ & $-1,02$ & 0,00 & $-1,89$ \\
\hline 132 & 19 & 60 & 111 & -54 & -98 & -39 & 64 & 38,76 & $\mathrm{~F}^{-}$ & - & $\mathrm{F}^{-}$ & $-0,58$ & 0,00 & $-0,58$ \\
\hline 133 & 22 & 66 & 141 & $-70-$ & -105 & -55 & 77 & 39,34 & $\mathrm{~F}^{-}$ & - & - & $-0,29$ & 0,00 & 0,00 \\
\hline
\end{tabular}


Apêndice 34. Índices DRIS, calculados pelo procedimento proposto por Jones (1981), diagnose para a correção de N, P e K, resposta líquida da produtividade, diagnose para o nutriente deficiente e a resposta da produtividade, para os dados, dos tratamentos com as doses 2 e 3 de $\mathrm{N}$ em todas as combinações de doses de $\mathrm{P}$ e $\mathrm{K}$, do ensaio de adubação $\mathrm{N}, \mathrm{P}$ e $\mathrm{K}$ conduzido em Araraquara/SP.

\begin{tabular}{|c|c|c|c|c|c|c|c|c|c|c|c|c|c|c|}
\hline \multirow[b]{2}{*}{ Trat. } & \multicolumn{6}{|c|}{ Índices DRIS } & \multirow[b]{2}{*}{$\mathrm{BN}$} & \multirow[b]{2}{*}{ Prod. } & \multicolumn{3}{|c|}{ Diagnose } & \multicolumn{3}{|c|}{ Resposta } \\
\hline & $\overline{\mathrm{N}}$ & $\mathrm{P}$ & $\mathrm{K}$ & $\mathrm{Ca}$ & $\mathrm{Mg}$ & $\bar{S}$ & & & $\mathrm{~N}$ & $P$ & $\mathrm{~K}$ & $d\left(Y_{N}\right)$ & $\mathrm{d}\left(\mathrm{Y}_{\mathrm{P}}\right)$ & $\bar{d}\left(Y_{K}\right)$ \\
\hline & & & & & & & & $\mathrm{tha}^{-1}$ & & & & & $-t h a^{-1}$ & \\
\hline 200 & 43 & 23 & 47 & -16 & -61 & -37 & 38 & 37,30 & $F^{-}$ & $\mathrm{V}^{-}$ & $\mathrm{F}^{-}$ & $-0,88$ & 0,58 & $-1,46$ \\
\hline 201 & 31 & 54 & 68 & -22 & -80 & -51 & 51 & 38,76 & $F^{-}$ & $\mathrm{V}^{-}$ & $\mathrm{F}^{-}$ & $-0,44$ & 0,29 & $-0,15$ \\
\hline 202 & 26 & 46 & 88 & -33 & -96 & -32 & 54 & 38,91 & $\mathrm{~F}^{-}$ & $\mathrm{F}^{-}$ & $\mathrm{V}^{-}$ & $-0,14$ & $-0,29$ & 0,88 \\
\hline 203 & 23 & 78 & 104 & -57 & -110 & -39 & 69 & 38,03 & $\mathrm{~V}^{-}$ & $\mathrm{F}^{-}$ & - & 0,29 & $-0,58$ & 0,00 \\
\hline 210 & 48 & 59 & 38 & -23 & -77 & -46 & 49 & 36,72 & $\mathrm{~F}^{-}$ & $\mathrm{V}^{-}$ & $\mathrm{F}^{-}$ & $-0,44$ & 0,73 & $-1,75$ \\
\hline 211 & 41 & 56 & 66 & -23 & -92 & -49 & 55 & 38,47 & $\mathrm{~F}^{-}$ & $\mathrm{V}^{-}$ & $\mathrm{F}^{-}$ & $-0,29$ & 0,29 & $-0,73$ \\
\hline 212 & 38 & 54 & 91 & -31 & -105 & -48 & 61 & 39,20 & $\mathrm{~V}^{*}$ & $\mathrm{~F}^{-}$ & $\mathrm{V}^{-}$ & 0,29 & $-0,14$ & 0,59 \\
\hline 213 & 42 & 54 & 117 & -52 & -116 & -45 & 71 & 38,61 & $\mathrm{~V}^{-}$ & $F^{*}$ & - & 0,58 & $-0,59$ & 0,00 \\
\hline 220 & 51 & 61 & 36 & -29 & -75 & -44 & 49 & 35,99 & $\mathrm{~F}^{-}$ & $\mathrm{V}^{*}$ & $\mathrm{~F}^{-}$ & $-0,15$ & 0,87 & $-2,19$ \\
\hline 221 & 42 & 58 & 65 & -30 & -90 & -46 & 55 & 38,18 & $\mathrm{~V}^{-}$ & $\mathrm{V}^{-}$ & $\mathrm{F}^{-}$ & 0,15 & 0,29 & $-1,16$ \\
\hline 222 & 37 & 56 & 94 & -40 & -102 & -45 & 62 & 39,34 & $\mathrm{~V}^{-}$ & $\mathrm{V}^{-}$ & $\mathrm{V}^{-}$ & 0,58 & 0,00 & 0,14 \\
\hline 223 & 43 & 62 & 124 & -59 & -109 & -62 & 77 & 39,20 & $\mathrm{~V}^{*}$ & $\mathrm{~F}^{-}$ & - & 0,88 & $-0,43$ & 0,00 \\
\hline 230 & 47 & 64 & 39 & -37 & -73 & -40 & 50 & 35,12 & $\mathrm{~V}^{-}$ & - & $\mathrm{F}^{-}$ & 0,00 & 0,00 & $-2,77$ \\
\hline 231 & 34 & 60 & 67 & -42 & -74 & -44 & 54 & 37,89 & $\mathrm{~V}^{-}$ & - & $\mathrm{F}^{-}$ & 0,44 & 0,00 & $-1,45$ \\
\hline 232 & 32 & 64 & 100 & -50 & -83 & -63 & 65 & 39,34 & $\mathrm{~V}^{*}$ & - & $F^{-}$ & 0,87 & 0,00 & $-0,29$ \\
\hline 233 & 30 & 65 & 131 & -75 & -92 & -59 & 75 & 39,63 & $\mathrm{~V}^{*}$ & - & - & 1,16 & 0,00 & 0,00 \\
\hline 300 & 31 & 54 & 22 & -26 & -54 & -26 & 36 & 38,18 & - & $\mathrm{V}^{*}$ & $\mathrm{~F}^{-}$ & 0,00 & 1,02 & $-1,02$ \\
\hline 301 & 16 & 80 & 44 & -37 & -61 & -42 & 47 & 39,20 & - & $\mathrm{V}^{-}$ & $\mathrm{V}^{*}$ & 0,00 & 0,44 & 0,15 \\
\hline 302 & 15 & 76 & 68 & -53 & -61 & -45 & 53 & 39,05 & - & $\mathrm{V}^{-}$ & $\mathrm{V}^{-}$ & 0,00 & 0,14 & 1,31 \\
\hline 303 & 5 & 97 & 87 & -87 & -66 & -36 & 63 & 37,74 & - & $\mathrm{F}^{-}$ & - & 0,00 & $-0,29$ & 0,00 \\
\hline 310 & 51 & 61 & 21 & -26 & -62 & -44 & 44 & 37,16 & - & $\mathrm{V}^{-}$ & $\mathrm{F}^{-}$ & 0,00 & 1,02 & $-1,60$ \\
\hline 311 & 28 & 82 & 40 & -41 & -71 & -38 & 50 & 38,76 & - & $\mathrm{V}^{-}$ & $\mathrm{F}^{-}$ & 0,00 & 0,73 & $-0,15$ \\
\hline 312 & 23 & 79 & 68 & -58 & -71 & -41 & 57 & 38,91 & - & $\mathrm{V}^{-}$ & $\mathrm{V}^{-}$ & 0,00 & 0,15 & 0,88 \\
\hline 313 & 22 & 78 & 97 & -86 & -69 & -41 & 66 & 38,03 & - & $\mathrm{F}^{-}$ & - & 0,00 & $-0,29$ & 0,00 \\
\hline 320 & 42 & 90 & 11 & -38 & -78 & -27 & 48 & 36,14 & - & $\mathrm{V}^{-}$ & $\mathrm{F}^{-}$ & 0,00 & 1,02 & $-1,89$ \\
\hline 321 & 36 & 91 & 45 & -43 & -77 & -52 & 57 & 38,03 & - & $\mathrm{V}^{*}$ & $\mathrm{~F}^{-}$ & 0,00 & 0,58 & $-0,73$ \\
\hline 322 & 26 & 87 & 72 & -63 & -65 & -57 & 62 & 38,76 & - & $\mathrm{V}^{*}$ & $\mathrm{~V}^{-}$ & 0,00 & 0,29 & 0,44 \\
\hline 323 & 26 & 88 & 103 & -90 & -74 & -53 & 72 & 38,32 & - & $\mathbf{F}^{-}$ & - & 0,00 & $-0,15$ & 0,00 \\
\hline 330 & 36 & 93 & 10 & -51 & -63 & -25 & 46 & 35,12 & - & - & $\mathrm{F}^{-}$ & 0,00 & 0,00 & $-2,33$ \\
\hline 331 & 21 & 89 & 44 & -60 & -65 & -29 & 51 & 37,45 & - & - & $\mathrm{F}^{-}$ & 0,00 & 0,00 & $-1,02$ \\
\hline 332 & 19 & 92 & 77 & -76 & -61 & -50 & 63 & 38,47 & - & - & $\mathrm{V}^{-}$ & 0,00 & 0,00 & 0,00 \\
\hline 333 & 13 & 92 & $109-$ & -108 & -58 & -47 & 71 & 38,47 & - & - & - & 0,00 & 0,00 & 0,00 \\
\hline
\end{tabular}


Apêndice 35. Índices DRIS, calculados pelo procedimento proposto por Jones (1981), diagnose para a correção de $\mathrm{N}, \mathrm{P}$ e K, resposta líquida da produtividade, diagnose para o nutriente deficiente e a resposta da produtividade, para os dados, dos tratamentos com as doses 0 e 1 de $\mathrm{N}$ em todas as combinações de doses de $P$ e $K$, do ensaio de adubação $\mathrm{N}, \mathrm{P}$ e K conduzido em Botucatu/SP.

\begin{tabular}{|c|c|c|c|c|c|c|c|c|c|c|c|c|c|}
\hline \multirow[b]{2}{*}{ Trat. } & \multicolumn{5}{|c|}{ Índices DRIS } & \multirow[b]{2}{*}{$\mathrm{BN}$} & \multirow[b]{2}{*}{ Prod. } & \multicolumn{3}{|c|}{ Diagnose } & \multicolumn{3}{|c|}{ Resposta } \\
\hline & $\overline{\mathrm{N}}$ & $\mathrm{P} \quad \mathrm{K}$ & $\mathrm{Ca}$ & $\mathrm{Mg}$ & $\overline{\mathrm{S}}$ & & & $\mathrm{N}$ & $\mathrm{P}$ & $\mathrm{K}$ & $\overline{d\left(Y_{N}\right)}$ & $d\left(Y_{P}\right)$ & $\mathrm{d}\left(\mathrm{Y}_{\mathrm{K}}\right)$ \\
\hline & & & & & & & tha- & & & & & 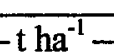 & \\
\hline 000 & -88 & $83-543$ & 68 & 417 & 62 & 210 & 36,04 & $\mathrm{~V}^{+}$ & $\mathrm{F}^{-}$ & $\mathrm{V}^{+}$ & 4,08 & $-0,76$ & 4,93 \\
\hline 001 & -139 & $77-165$ & 35 & 234 & -41 & 115 & 40,97 & $\mathrm{~V}^{+}$ & $\mathrm{F}^{-}$ & $\mathrm{V}^{+}$ & 4,42 & $-0,76$ & 2,29 \\
\hline 002 & -156 & $\begin{array}{ll}73 & 27\end{array}$ & -37 & 136 & -42 & 79 & 43,26 & $\mathrm{~V}^{+}$ & $\mathrm{F}^{-}$ & $\mathrm{V}^{-}$ & 4,77 & $-0,69$ & 0,42 \\
\hline 003 & -146 & 50167. & 154 & 70 & 13 & 100 & 42,84 & $\mathrm{~V}^{+}$ & $F^{-}$ & - & 5,10 & $-0,68$ & 0,00 \\
\hline 010 & -143 & $93-596$ & 82 & 461 & 103 & 246 & 36,80 & $\mathrm{~V}^{+}$ & $\mathrm{F}^{-}$ & $\mathrm{V}^{+}$ & 4,51 & $-1,71$ & 4,93 \\
\hline 011 & -200 & $82-206$ & 51 & 265 & 9 & 136 & 41,73 & $\mathrm{~V}^{+}$ & $\mathrm{F}^{-}$ & $\mathrm{V}^{+}$ & 4,85 & $-1,71$ & 2,22 \\
\hline 012 & -208 & $47 \quad-5$ & -3 & 160 & 9 & 72 & 43,95 & $\mathrm{~V}^{+}$ & $\mathrm{F}^{-}$ & $\mathrm{F}^{+}$ & 5,27 & $-1,70$ & $-0,43$ \\
\hline 013 & -199 & $64 \quad 127$. & 103 & 87 & 23 & 101 & 43,52 & $\mathrm{~V}^{+}$ & $F^{*}$ & - & 5,52 & $-1,61$ & 0,00 \\
\hline 020 & -167 & $76-666$ & 92 & 507 & 158 & 278 & 38,51 & $\mathrm{~V}^{+}$ & $F^{*}$ & $\mathrm{~V}^{+}$ & 5,01 & $-2,72$ & 4,93 \\
\hline 021 & -225 & $69-242$ & 68 & 299 & 30 & 156 & 43,44 & $\mathrm{~V}^{+}$ & $\mathrm{F}^{-}$ & $\mathrm{V}^{+}$ & 5,35 & $-2,72$ & 2,21 \\
\hline 022 & -235 & $68-41$ & 13 & 182 & 12 & 92 & 45,65 & $\mathrm{~V}^{+}$ & $\mathrm{F}^{-}$ & $\mathrm{F}^{+}$ & 5,69 & $-2,72$ & $-0,52$ \\
\hline 023 & -218 & $54 \quad 99$ & -69 & 103 & 32 & 96 & 45,13 & $\mathrm{~V}^{+}$ & $\mathrm{F}^{-}$ & - & 6,04 & $-2,72$ & 0,00 \\
\hline 030 & -143 & $73-813$ & 103 & 579 & 200 & 319 & 41,23 & $\mathrm{~V}^{+}$ & - & $\mathrm{V}^{+}$ & 5,43 & 0,00 & 4,93 \\
\hline 031 & -225 & $61-303$ & 77 & 329 & 60 & 176 & 46 & $\mathrm{~V}^{+}$ & - & $\mathrm{V}^{+}$ & 5,78 & 0,00 & 2,21 \\
\hline 032 & -238 & $65-72$ & 28 & 203 & 14 & 103 & 48,37 & $\mathrm{~V}^{+}$ & - & $\mathrm{F}^{+}$ & 6,12 & 0,00 & $-0,52$ \\
\hline 033 & -220 & $49 \quad 74$ & -49 & 119 & 27 & 90 & 47,85 & $\mathrm{~V}^{+}$ & - & - & 6,46 & 0,00 & 0,00 \\
\hline 100 & 34 & $78-831$ & 81 & 541 & 98 & 277 & 40,12 & $\mathrm{~F}^{-}$ & $\mathrm{F}^{-}$ & $\mathrm{V}^{+}$ & $-1,28$ & $-1,19$ & 5,27 \\
\hline 101 & -71 & $65-318$ & 50 & 301 & -28 & 139 & 45,39 & $\mathrm{~V}^{+}$ & $\mathrm{F}^{-}$ & $\mathrm{V}^{+}$ & 1,70 & $-1,19$ & 2,64 \\
\hline 102 & -106 & $\begin{array}{ll}63 & -80\end{array}$ & -13 & 182 & -46 & 82 & 48,03 & $\mathrm{~V}^{+}$ & $\mathrm{F}^{-}$ & $\mathrm{F}^{+}$ & 2,03 & $-1,19$ & $-0,09$ \\
\hline 103 & -112 & $76 \quad 63-$ & 118 & 103 & -12 & 81 & 47,94 & $\mathrm{~V}^{+}$ & $F$ & - & 2,38 & $-1,10$ & 0,00 \\
\hline 110 & -18 & $91-865$ & 92 & 557 & 144 & 295 & 41,31 & $\mathrm{~V}^{+}$ & $\mathrm{F}^{-}$ & $\mathrm{V}^{+}$ & 1,78 & $-2,21$ & 5,27 \\
\hline 111 & -123 & $80-342$ & 66 & 312 & 7 & 155 & 46,58 & $\mathrm{~V}^{+}$ & $\mathrm{F}^{-}$ & $\mathrm{V}^{+}$ & 2,13 & $-2,21$ & 2,64 \\
\hline 112 & -152 & $78-108$ & 10 & 191 & -19 & 93 & 49,22 & $\mathrm{~V}^{+}$ & $F^{-}$ & $\mathrm{F}^{+}$ & 2,37 & $-2,12$ & $-0,18$ \\
\hline 113 & -148 & $\begin{array}{ll}61 & 38\end{array}$ & -72 & 126 & -5 & 75 & 49,04 & $\mathrm{~V}^{+}$ & $\mathrm{F}^{-}$ & - & 2,81 & $-2,13$ & 0,00 \\
\hline 120 & -27 & $81-951$ & 103 & 605 & 188 & 326 & 43,52 & $\mathrm{~V}^{+}$ & $\mathrm{F}^{-}$ & $\mathrm{V}^{+}$ & 2,21 & $-3,14$ & 5,27 \\
\hline 121 & -142 & $99-390$ & 73 & 333 & 27 & 177 & 48,79 & $\mathrm{~V}^{+}$ & $\mathrm{F}^{-}$ & $\mathrm{V}^{+}$ & 2,55 & $-3,15$ & 2,55 \\
\hline 122 & -163 & $72-130$ & 31 & 210 & -21 & 105 & 51,34 & $\mathrm{~V}^{+}$ & $\mathrm{F}^{-}$ & $\mathrm{F}^{+}$ & 2,89 & $-3,15$ & $-0,17$ \\
\hline 123 & -169 & $83 \quad 12$ & -51 & 126 & -1 & 74 & 51,17 & $\mathrm{~V}^{+}$ & $\mathrm{F}^{-}$ & - & 3,32 & $-3,14$ & 0,00 \\
\hline 130 & 23 & $109-1149$ & 106 & 699 & 211 & 383 & 46,66 & $\mathrm{~F}^{-}$ & - & $\mathrm{V}^{+}$ & $-2,64$ & 0,00 & 5,28 \\
\hline 131 & -121 & $102-445$ & 85 & 361 & 19 & 189 & 51,94 & $\mathrm{~V}^{+}$ & - & $\mathrm{V}^{+}$ & 2,97 & 0,00 & 2,55 \\
\hline 132 & -161 & $100-171$ & 37 & 219 & -24 & 119 & 54,49 & $\mathrm{~V}^{+}$ & - & $\mathrm{F}^{+}$ & 3,31 & 0,00 & $-0,18$ \\
\hline 133 & -160 & $86-10$ & -33 & 137 & -19 & 74 & 54,31 & $\mathrm{~V}^{+}$ & - & - & 3,66 & 0,00 & 0,00 \\
\hline
\end{tabular}


Apêndice 36. Índices DRIS, calculados pelo procedimento proposto por Jones (1981), diagnose para a correção de N, P e K, resposta líquida da produtividade, diagnose para o nutriente deficiente e a resposta da produtividade, para os dados, dos tratamentos com as doses 2 e 3 de $\mathrm{N}$ em todas as combinações de doses de $\mathrm{P}$ e $\mathrm{K}$, do ensaio de adubação $\mathrm{N}, \mathrm{P}$ e K conduzido em Botucatu/SP.

\begin{tabular}{|c|c|c|c|c|c|c|c|c|c|c|c|c|c|}
\hline \multirow[b]{2}{*}{ Trat. } & \multicolumn{5}{|c|}{ Índices DRIS } & \multirow[b]{2}{*}{$\mathrm{BN}$} & \multirow[b]{2}{*}{ Prod. } & \multicolumn{3}{|c|}{ Diagnose } & \multicolumn{3}{|c|}{ Resposta } \\
\hline & $\overline{\mathrm{N}}$ & $\mathrm{K}$ & $\mathrm{Ca}$ & $\mathrm{Mg}$ & $\bar{S}$ & & & $\mathrm{~N}$ & $P$ & $\mathbf{K}$ & $d\left(Y_{N}\right)$ & $d\left(Y_{P}\right)$ & $\mathrm{d}\left(\mathrm{Y}_{\mathrm{K}}\right)$ \\
\hline & & & & & & & $t h a^{-1}$ & & & & & - tha ${ }^{-1}$ & \\
\hline 200 & 132 & $75-1060$ & 89 & 648 & 116 & 353 & 41,40 & $\mathrm{~V}^{-}$ & $F^{-}$ & $\mathrm{V}^{+}$ & 1,45 & $-1,69$ & 5,69 \\
\hline 201 & -7 & $62-448$ & 61 & 368 & -35 & 164 & 47,09 & $\mathrm{~F}^{+}$ & $\mathrm{F}^{-}$ & $\mathrm{V}^{+}$ & $-1,19$ & $-1,62$ & 2,97 \\
\hline 202 & -67 & $92-177$ & -3 & 229 & -75 & 107 & 50,06 & $\mathrm{~F}^{+}$ & $F^{-}$ & $\mathrm{V}^{+}$ & $-0,84$ & $-1,53$ & 0,26 \\
\hline 203 & -71 & $76-15$ & -88 & 156 & -59 & 78 & 50,32 & $\mathrm{~F}^{+}$ & $\mathrm{F}^{-}$ & - & $-0,51$ & $-1,53$ & 0,00 \\
\hline 210 & 71 & $92-1043$ & 99 & 652 & 128 & 348 & 43,09 & $\mathrm{~V}^{-}$ & $\mathrm{F}^{-}$ & $\mathrm{V}^{+}$ & 1,10 & $-2,64$ & 5,62 \\
\hline 211 & -57 & $110-468$ & 69 & 373 & -27 & 184 & 48,71 & $\mathrm{~F}^{+}$ & $\mathrm{F}^{-}$ & $\mathrm{V}^{+}$ & $-0,68$ & $-2,63$ & 2,88 \\
\hline 212 & -104 & $111-196$ & 20 & 232 & -62 & 121 & 51,59 & $\mathrm{~F}^{+}$ & $\mathrm{F}^{-}$ & $\mathrm{V}^{+}$ & $-0,33$ & $-2,64$ & 0,26 \\
\hline 213 & -113 & $91-40$ & -57 & 155 & -35 & 82 & 51,85 & $\mathrm{~F}^{+}$ & - & - & 00 & $-2,64$ & 0,00 \\
\hline 220 & 77 & $115-1133$ & 104 & 691 & 145 & 378 & 45,73 & $\mathrm{~V}^{-}$ & $\mathbf{F}$ & $\mathrm{V}^{+}$ & 0,60 & $-3,57$ & 5,61 \\
\hline 221 & -60 & $108-495$ & 84 & 382 & -20 & 192 & 51,34 & $\mathrm{~F}^{+}$ & $\mathrm{F}^{-}$ & $\mathrm{V}^{+}$ & $-0,25$ & $-3,57$ & 2,89 \\
\hline 222 & -108 & $108-211$ & 38 & 233 & -61 & 127 & 54,23 & $\mathrm{~V}^{+}$ & $\mathrm{F}^{-}$ & $\mathrm{V}^{+}$ & 0,08 & $-3,57$ & 0,26 \\
\hline 223 & -117 & $119-56$ & -34 & 151 & -63 & 90 & 54,49 & $\mathrm{~V}^{+}$ & $\mathrm{F}^{-}$ & - & 0,42 & $-3,48$ & 0,00 \\
\hline 230 & 149 & $120-1324$ & 113 & 774 & 169 & 442 & 49,30 & $\mathrm{~V}^{-}$ & - & $\mathrm{V}^{+}$ & 0,08 & 0,00 & 5,61 \\
\hline 231 & -34 & $138-550$ & 87 & 396 & -38 & 207 & 54,91 & $\mathrm{~V}^{+}$ & - & $\mathrm{V}^{+}$ & 0,25 & 0,00 & 2,89 \\
\hline 232 & -83 & $142-237$ & 46 & 237 & -106 & 142 & 57,80 & $\mathrm{~V}^{+}$ & - & $\mathrm{V}^{+}$ & 0,60 & 0,00 & 0,17 \\
\hline 233 & -101 & $124-74$ & -21 & 154 & -81 & 93 & 57,97 & $\mathrm{~V}^{+}$ & - & - & 0,94 & 0,00 & 0,00 \\
\hline 300 & 175 & 80-1091 & 96 & 717 & 21 & 363 & 39,95 & - & $\mathbf{F}^{-}$ & $\mathrm{V}^{+}$ & 0,00 & $-2,04$ & 5,95 \\
\hline 301 & 38 & $101-502$ & 65 & 420 - & -121 & 208 & 45,90 & - & $\mathrm{F}^{-}$ & $\mathrm{V}^{+}$ & 0,00 & $-2,13$ & 3,32 \\
\hline 302 & -14 & $100-236$ & 13 & $280-$ & -143 & 131 & 49,22 & - & $\mathrm{F}^{-}$ & $\mathrm{V}^{+}$ & 0,00 & $-2,04$ & 0,59 \\
\hline 303 & -44 & $108-78$ & -73 & $198-$ & -111 & 102 & 49,81 & - & $\mathrm{F}^{-}$ & - & 0,00 & $-2,04$ & 0,00 \\
\hline 310 & 104 & $101-1016$ & 105 & 677 & 30 & 339 & 41,99 & - & $\mathrm{F}^{-}$ & $\mathrm{V}^{+}$ & 0,00 & $-3,14$ & 6,04 \\
\hline 311 & -7 & $119-496$ & 77 & 407 & -100 & 201 & 48,03 & - & $\mathrm{F}^{-}$ & $\mathrm{V}^{+}$ & 0,00 & $-3,06$ & 3,23 \\
\hline 312 & -55 & $119-238$ & 31 & 270 . & 126 & 140 & 51,26 & - & $F^{-}$ & $\mathrm{V}^{+}$ & 0,00 & $-3,05$ & 0,59 \\
\hline 313 & -78 & $125-90$ & -46 & $190-$ & -101 & 105 & 51,85 & - & $F^{*}$ & - & 0,00 & $-3,06$ & 0,00 \\
\hline 320 & 109 & $129-1038$ & 111 & 687 & 2 & 346 & 45,13 & - & $F^{-}$ & $\mathrm{V}^{+}$ & 0,00 & $-4,09$ & 5,96 \\
\hline 321 & -2 & $147-516$ & 86 & $413-$ & -127 & 215 & 51,09 & - & $F^{-}$ & $\mathrm{V}^{+}$ & 0,00 & $-4,07$ & 3,22 \\
\hline 322 & -51 & $146-251$ & 43 & $268-$ & -155 & 152 & 54,31 & - & $F^{-}$ & $\mathrm{V}^{+}$ & 0,00 & $-4,09$ & 0,60 \\
\hline 323 & -75 & $152-103$ & -26 & $180-$ & -127 & 111 & 54,91 & - & $F^{-}$ & - & 0,00 & $-4,00$ & 0,00 \\
\hline 330 & 180 & $161-1133$ & 113 & 724 & -45 & 393 & 49,22 & - & - & $\mathrm{V}^{+}$ & 0,00 & 0,00 & 5,94 \\
\hline 331 & 46 & $177-543$ & 90 & 414 . & -185 & 243 & 55,16 & - & - & $\mathrm{V}^{+}$ & 0,00 & 0,00 & 3,24 \\
\hline 332 & -10 & $178-265$ & 51 & $254-$ & -208 & 161 & 58,40 & - & - & $\mathrm{V}^{+}$ & 0,00 & 0,00 & 0,51 \\
\hline 333 & -40 & $161-116$ & -12 & $177-$ & -169 & 113 & 58,91 & - & - & - & 0,00 & 0,00 & 0,00 \\
\hline
\end{tabular}


Apêndice 37. Índices DRIS, calculados pelo procedimento proposto por Jones (1981), diagnose para a correção de $\mathrm{N}, \mathrm{P}$ e K , resposta líquida da produtividade, diagnose para o nutriente deficiente e a resposta da produtividade, para os dados, dos tratamentos com as doses 0 e $1 \mathrm{de} \mathrm{N}$ em todas as combinações de doses de $\mathrm{P}$ e $\mathrm{K}$, do ensaio de adubação $\mathrm{N}, \mathrm{P}$ e $\mathrm{K}$ conduzido em Matão/SP.

\begin{tabular}{|c|c|c|c|c|c|c|c|c|c|c|c|c|c|c|}
\hline \multirow[b]{2}{*}{ Trat. } & \multicolumn{6}{|c|}{ Índices DRIS } & \multirow[b]{2}{*}{$\mathrm{BN}$} & \multirow[b]{2}{*}{ Prod. } & \multicolumn{3}{|c|}{ Diagnose } & \multicolumn{3}{|c|}{ Resposta } \\
\hline & $\overline{\mathrm{N}}$ & $\overline{\mathrm{p}}$ & $\mathrm{K}$ & $\mathrm{Ca}$ & $\overline{\mathrm{Mg}}$ & $\bar{S}$ & & & $\mathrm{~N}$ & $\mathrm{P}$ & $\mathrm{K}$ & $\mathrm{d}\left(\mathrm{Y}_{\mathrm{N}}\right)$ & $\mathrm{d}\left(\mathrm{Y}_{\mathrm{P}}\right)$ & $\mathrm{d}\left(\mathrm{Y}_{\mathrm{K}}\right)$ \\
\hline & & & & & & & & $\mathrm{tha}^{-1}$ & & & & & na & \\
\hline 000 & -83 & -21 & 45 & 7 & 98 & -46 & 50 & 34,77 & $\mathrm{~V}^{+}$ & $\mathrm{V}^{+}$ & $\mathrm{V}^{*}$ & 2,21 & 4,75 & 1,54 \\
\hline 001 & -91 & 0 & 99 & -28 & 72 & -52 & 57 & 33,23 & $\mathrm{~V}^{+}$ & $\mathrm{F}^{-}$ & $\mathrm{F}^{-}$ & 2,04 & -4.34 & $-0,35$ \\
\hline 002 & -76 & 1 & 154 & -52 & 45 & -72 & 67 & 33,58 & $\mathrm{~V}^{+}$ & $\mathrm{F}^{-}$ & $\mathrm{F}^{-}$ & 1,95 & $-3,90$ & $-2,21$ \\
\hline 003 & -55 & 15 & 201 & -77 & 17. & -101 & 78 & 35,79 & $\mathrm{~V}^{+}$ & $\mathrm{F}^{-}$ & - & 1,86 & $-3,48$ & 0,00 \\
\hline 010 & -98 & 9 & 59 & -1 & 95 & -64 & 54 & 39,52 & $\mathrm{~V}^{+}$ & $\mathrm{F}^{-}$ & $\mathrm{V}^{-}$ & 2,30 & $-2,47$ & 1,95 \\
\hline 011 & -83 & 0 & 109 & -21 & 69 & -75 & 60 & 37,57 & $\mathrm{~V}^{+}$ & $F^{-}$ & $\mathrm{V}^{-}$ & 2,13 & $-2,04$ & 0,09 \\
\hline 012 & -80 & 31 & 141 & -52 & 45 & -86 & 73 & 37,48 & $\mathrm{~V}^{+}$ & $F^{-}$ & $\mathrm{F}^{-}$ & 2,04 & $-1,54$ & $-1,79$ \\
\hline 013 & -56 & 46 & 179 & -74 & 19. & -114 & 81 & 39,27 & $\mathrm{~V}^{+}$ & $F^{-}$ & - & 1,96 & $-1,11$ & 0,00 \\
\hline 020 & -99 & 6 & 60 & 8 & 93 & -67 & 56 & 41,99 & $\mathrm{~V}^{+}$ & $F^{-}$ & $\mathrm{V}^{-}$ & 2,38 & $-0,09$ & 2,38 \\
\hline 021 & -88 & -8 & 95 & -13 & 74 & -60 & 56 & 39,61 & $\mathrm{~V}^{+}$ & $\mathrm{F}^{+}$ & $\mathrm{V}^{-}$ & 2,21 & $-0,34$ & 0,59 \\
\hline 022 & -80 & 26 & 115 & -41 & 51 & -71 & 64 & 39,02 & $\mathrm{~V}^{+}$ & $\mathrm{V}^{-}$ & $\mathrm{F}^{-}$ & 2,12 & 0,77 & $-1,36$ \\
\hline 023 & -54 & 43 & 145 & -59 & 24 & -99 & 71 & 40,38 & $\mathrm{~V}^{+}$ & $\mathrm{V}^{*}$ & - & 2,03 & 1,19 & 0,00 \\
\hline 030 & -99 & -41 & 44 & 29 & 106 & -39 & 60 & 42,08 & $\mathrm{~V}^{+}$ & - & $\mathrm{V}^{-}$ & 2,54 & 0,00 & 2,81 \\
\hline 031 & -89 & -12 & 59 & 2 & 82 & -42 & 48 & 39,27 & $\mathrm{~V}^{+}$ & - & $\mathrm{V}^{-}$ & 2,29 & 0,00 & 1,02 \\
\hline 032 & -71 & -11 & 78 & -16 & 63 & -43 & 47 & 38,25 & $\mathrm{~V}^{+}$ & - & $\mathrm{F}^{-}$ & 2,29 & 0,00 & $-0,94$ \\
\hline 033 & -48 & 2 & 93 & -36 & 43 & -54 & 46 & 39,19 & $\mathrm{~V}^{+}$ & - & - & 2,12 & 0.00 & 0,00 \\
\hline 100 & -28 & 26 & -27 & -3 & 104 & -73 & 44 & 36,98 & $\mathrm{~V}^{+}$ & $\mathrm{F}^{-}$ & $\mathrm{F}^{+}$ & 1,01 & $-4,84$ & $-1,71$ \\
\hline 101 & -37 & 7 & 48 & -27 & 81 & .72 & 45 & 35,27 & $\mathrm{~V}^{+}$ & $\mathrm{F}^{-}$ & $F^{-}$ & 0,94 & $-4,43$ & $-0,26$ \\
\hline 102 & -35 & 2 & 110 & -50 & 50 & -77 & 54 & 35,53 & $\mathrm{~V}^{+}$ & $\mathrm{F}^{-}$ & $\mathrm{F}^{-}$ & 0,85 & $-3,99$ & $-2,12$ \\
\hline 103 & -23 & 13 & 163 & -73 & $30-$ & -110 & 69 & 37,65 & $\mathrm{~V}^{+}$ & $\mathrm{F}^{-}$ & - & 0,69 & $-3,58$ & 0,00 \\
\hline 110 & -33 & 22 & 15 & -1 & 98 - & -102 & 45 & 41,82 & $\mathrm{~V}^{+}$ & $\mathrm{F}^{-}$ & $\mathrm{V}^{-}$ & 1,19 & $-2,55$ & 2,12 \\
\hline 111 & -41 & 1 & 68 & -26 & 76 & -78 & 48 & 39,70 & $\mathrm{~V}^{+}$ & $\mathrm{F}^{-}$ & $\mathrm{V}^{-}$ & 1,10 & $-2,12$ & 0,18 \\
\hline 112 & -36 & 35 & 109 & -51 & 52. & -109 & 65 & 39,52 & $\mathrm{~V}^{+}$ & $F^{-}$ & $\mathrm{F}^{-}$ & 0,94 & $-1,62$ & $-1,71$ \\
\hline 113 & -17 & 11 & 158 & -67 & $28-$ & -113 & 66 & 41,23 & $\mathrm{~V}^{+}$ & $\mathrm{F}^{-}$ & - & 0,76 & $-1,18$ & 0,00 \\
\hline 120 & -47 & 13 & 25 & 2 & 93 & -87 & 45 & 44,37 & $\mathrm{~V}^{+}$ & $\mathrm{F}^{-}$ & $\mathrm{V}^{-}$ & 1,28 & $-0,25$ & 2,55 \\
\hline 121 & -42 & -2 & 66 & -17 & 74 & -80 & 47 & 41,82 & $\mathrm{~V}^{+}$ & $\mathrm{F}^{+}$ & $\mathrm{V}^{*}$ & 1,19 & $-0,26$ & 0,68 \\
\hline 122 & -39 & 30 & 93 & -42 & 50 & -92 & 58 & 41,14 & $\mathrm{~V}^{+}$ & $\mathrm{V}^{-}$ & $\mathrm{F}^{-}$ & 1,10 & 0,60 & $-1,27$ \\
\hline 123 & -25 & 40 & 124 & -62 & 29. & -106 & 64 & 42,41 & $\mathrm{~V}^{+}$ & $\mathrm{V}^{-}$ & - & 0,94 & 1,10 & 0,00 \\
\hline 130 & -47 & -32 & 18 & 21 & 98 & -57 & 46 & 44,62 & $\mathrm{~V}^{+}$ & - & $\mathrm{V}^{-}$ & 1,37 & 0,00 & 3,06 \\
\hline 131 & -52 & -12 & 36 & -7 & 80 & -45 & 39 & 41,56 & $\mathrm{~V}^{+}$ & - & $\mathrm{V}^{-}$ & 1,28 & 0,00 & 1,02 \\
\hline 132 & -39 & -13 & 62 & -23 & 60 & -47 & 41 & 40,54 & $\mathrm{~V}^{+}$ & - & $F^{-}$ & 1,11 & 0,00 & $-0,77$ \\
\hline 133 & -22 & 0 & 82 & -40 & 40 & -59 & 41 & 41,31 & $\mathrm{~V}^{+}$ & _- & - & 1,02 & 0,00 & 0,00 \\
\hline
\end{tabular}


Apêndice 38. Índices DRIS, calculados pelo procedimento proposto por Jones (1981), diagnose para a correção de $\mathrm{N}, \mathrm{P}$ e K, resposta líquida da produtividade, diagnose para o nutriente deficiente e a resposta da produtividade, para os dados, dos tratamentos com as doses 2 e 3 de $\mathrm{N}$ em todas as combinações de doses de $\mathrm{P}$ e $\mathrm{K}$, do ensaio de adubação $\mathrm{N}, \mathrm{P}$ e $\mathrm{K}$ conduzido em Matão/SP.

\begin{tabular}{|c|c|c|c|c|c|c|c|c|c|c|c|c|c|c|}
\hline \multirow[b]{2}{*}{ Trat. } & \multicolumn{6}{|c|}{ Índices DRIS } & \multirow[b]{2}{*}{$\mathrm{BN}$} & \multirow[b]{2}{*}{ Prod. } & \multicolumn{3}{|c|}{ Diagnose } & \multicolumn{3}{|c|}{ Resposta } \\
\hline & $\overline{\mathrm{N}}$ & $P$ & $\mathrm{~K}$ & $\mathrm{Ca}$ & $\mathrm{Mg}$ & $\bar{S}$ & & & $\mathrm{~N}$ & $P$ & $\mathrm{~K}$ & $\mathrm{~d}\left(\mathrm{Y}_{\mathrm{N}}\right)$ & $d\left(Y_{P}\right)$ & $\mathrm{d}\left(\mathrm{Y}_{\mathrm{K}}\right)$ \\
\hline & & & & & & & & $\mathrm{tha}^{-1}$ & & & & & $\overline{\text { ha }}$ & \\
\hline 200 & 22 & 27 & -71 & -10 & 106 & -73 & 52 & 37,99 & $\mathrm{~V}^{-}$ & $F^{-}$ & $\mathrm{F}^{+}$ & 0,00 & $-5,02$ & $-1,78$ \\
\hline 201 & 3 & 6 & 20 & -32 & 79 & -75 & 36 & 36,21 & $\mathrm{~V}^{-}$ & $F^{-}$ & $\mathrm{F}^{-}$ & 0,09 & $-4,59$ & $-0,17$ \\
\hline 202 & 3 & 3 & 92 & -52 & 57 & -103 & 52 & 36,38 & $\mathrm{~V}^{-}$ & $F^{-}$ & $\mathrm{F}^{-}$ & 0,26 & $-4,08$ & $-1,96$ \\
\hline 203 & 14 & -32 & 155 & -68 & 40 & -109 & 70 & 38,34 & $\mathrm{~V}^{-}$ & $\mathrm{V}^{+}$ & - & 0,35 & 3,65 & 0,00 \\
\hline 210 & 12 & 23 & -7 & -12 & 89 & -105 & 41 & 43,01 & $\mathrm{~F}^{-}$ & $F^{-}$ & $\mathrm{F}^{+}$ & $-0,08$ & $-2,64$ & $-2,21$ \\
\hline 211 & 3 & 3 & 54 & -32 & 74 & -102 & 45 & 40,80 & $\mathrm{~V}^{-}$ & $F^{-}$ & $\mathrm{V}^{-}$ & 0,00 & $-2,21$ & 0,34 \\
\hline 212 & 3 & -3 & 105 & -51 & 54 & -107 & 54 & 40,46 & $\mathrm{~V}^{-}$ & $\mathrm{V}^{+}$ & $\mathrm{F}^{-}$ & 0,17 & 1,78 & $-1,53$ \\
\hline 213 & 8 & 4 & 151 & -73 & 32 & -122 & 65 & 41,99 & $\mathrm{~V}^{-}$ & $F^{-}$ & - & 0,26 & $-1,36$ & 0,00 \\
\hline 220 & -5 & 14 & 16 & -10 & 74 & -90 & 35 & 45,65 & $\mathrm{~V}^{+}$ & $F^{-}$ & $\mathrm{V}^{-}$ & 0,16 & $-0,34$ & 2,64 \\
\hline 221 & -8 & -4 & 62 & -27 & 63 & -85 & 42 & 43,01 & $\mathrm{~V}^{+}$ & $\mathrm{F}^{+}$ & $\mathrm{V}^{-}$ & 0,08 & $-0,17$ & 0,77 \\
\hline 222 & -4 & -10 & 100 & -45 & 50 & -91 & 50 & 42,24 & $\mathrm{~F}^{+}$ & $\mathrm{F}^{+}$ & $\mathrm{F}^{-}$ & $-0,08$ & $-0,59$ & $-1,11$ \\
\hline 223 & 6 & -1 & 133 & -63 & 29 & -105 & 56 & 43,35 & $\mathrm{~V}^{-}$ & $\mathrm{F}^{+}$ & - & 0,17 & $-1,02$ & 0,00 \\
\hline 230 & -9 & -32 & 18 & 6 & 78 & -61 & 34 & 45,99 & $\mathrm{~V}^{+}$ & - & $\mathrm{V}^{-}$ & 0,25 & 0,00 & 3,15 \\
\hline 231 & -22 & -13 & 42 & -19 & 59 & -48 & 34 & 42,84 & $\mathrm{~V}^{+}$ & - & $\mathrm{V}^{-}$ & 0,17 & 0,00 & 1,19 \\
\hline 232 & -14 & -16 & 69 & -34 & 48 & -52 & 39 & 41,65 & $\mathrm{~F}^{+}$ & - & $\mathrm{F}^{-}$ & 0,00 & 0,00 & $-0,68$ \\
\hline 233 & 1 & -6 & 93 & -48 & 26 & -66 & 40 & 42,33 & $\mathrm{~V}^{*}$ & - & - & 0,09 & 0,00 & 0,00 \\
\hline 300 & 62 & 24 & -75 & -24 & 93 & -80 & 60 & 37,99 & - & $F^{-}$ & $\mathrm{F}^{+}$ & 0,00 & $-5,10$ & $-1,87$ \\
\hline 301 & 33 & 1 & 18 & -43 & 74 & -83 & 42 & 36,12 & - & $\mathrm{F}^{*}$ & $\mathrm{~V}^{-}$ & 0,00 & $-4,68$ & 0,00 \\
\hline 302 & 32 & -50 & 98 & -55 & 56 & -81 & 62 & 36,12 & - & $\mathrm{V}^{+}$ & $\mathrm{F}^{-}$ & 0,00 & 4,17 & $-1,87$ \\
\hline 303 & 29 & -46 & 160 & -76 & 33 & -99 & 74 & 37,99 & - & $\mathrm{V}^{+}$ & - & 0,00 & 3,74 & 0,00 \\
\hline 310 & 38 & 15 & -3 & -31 & 74 & -93 & 42 & 43,09 & - & $F^{-}$ & $\mathrm{F}^{+}$ & 0,00 & $-2,72$ & $-2,29$ \\
\hline 311 & 22 & -7 & 63 & -48 & 60 & -90 & 48 & 40,80 & - & $\mathrm{V}^{+}$ & $\mathrm{V}^{-}$ & 0,00 & 2,29 & 0,51 \\
\hline 312 & 18 & -14 & 118 & -65 & 39 & -96 & 58 & 40,29 & - & $\mathrm{V}^{+}$ & $\mathrm{F}^{-}$ & 0,00 & 1,87 & $-1,44$ \\
\hline 313 & 32 & -50 & 173 & -76 & 22 & -101 & 76 & 41,73 & - & $\mathrm{V}^{+}$ & - & 0,00 & 1,45 & 0,00 \\
\hline 320 & 26 & 11 & 34 & -27 & 52 & -97 & 41 & 45,81 & - & $F^{-}$ & $\mathrm{V}^{-}$ & 0,00 & $-0,43$ & 2,72 \\
\hline 321 & 12 & -13 & 80 & -45 & 39 & -74 & 44 & 43,09 & - & $\mathrm{F}^{+}$ & $\mathrm{V}^{-}$ & 0,00 & $-0,08$ & 0,93 \\
\hline 322 & 11 & -20 & 119 & -60 & 28 & -78 & 53 & 42,16 & - & $\mathrm{F}^{+}$ & $\mathrm{F}^{-}$ & 0,00 & $-0,51$ & $-1,02$ \\
\hline 323 & 25 & -56 & 164 & -69 & 19 & -83 & 69 & 43,18 & - & $\mathrm{F}^{+}$ & - & 0,00 & $-0,94$ & 0,00 \\
\hline 330 & 14 & -40 & 47 & -15 & 44 & -50 & 35 & 46,24 & - & - & $\mathrm{V}^{-}$ & 0,00 & 0,00 & 3,23 \\
\hline 331 & 5 & -67 & 81 & -33 & 41 & -27 & 42 & 43,01 & - & - & $\mathrm{V}^{-}$ & 0,00 & 0,00 & 1,36 \\
\hline 332 & 7 & -71 & 109 & -44 & 30 & -30 & 49 & 41,65 & - & - & $\mathrm{F}^{*}$ & 0,00 & 0,00 & $-0,59$ \\
\hline 333 & 13 & -66 & 133 & -59 & 5 & -26 & 50 & 42,24 & - & - & - & 0,00 & 0,00 & 0,00 \\
\hline
\end{tabular}


Apêndice 39. Índices DRIS, calculados pelo procedimento proposto por Jones (1981), diagnose para a correção de N, P e K, resposta líquida da produtividade, diagnose para o nutriente deficiente e a resposta da produtividade, para os dados, dos tratamentos com as doses 0 e $1 \mathrm{de} \mathrm{N}$ em todas as combinações de doses de $\mathrm{P}$ e $\mathrm{K}$, do ensaio de adubação $\mathrm{N}, \mathrm{P}$ e $\mathrm{K}$ conduzido em Monte Azul/SP.

\begin{tabular}{|c|c|c|c|c|c|c|c|c|c|c|c|c|c|c|}
\hline \multirow[b]{2}{*}{ Trat. } & \multicolumn{6}{|c|}{ Índices DRIS } & \multirow[b]{2}{*}{$\mathrm{BN}$} & \multirow[b]{2}{*}{ Prod. } & \multicolumn{3}{|c|}{ Diagnose } & \multicolumn{3}{|c|}{ Resposta } \\
\hline & $\overline{\mathrm{N}}$ & $\mathrm{P}$ & $\mathrm{K}$ & $\mathrm{Ca}$ & $\mathrm{Mg}$ & $\overline{\mathrm{S}}$ & & & $\mathrm{N}$ & $P$ & $\mathrm{~K}$ & $d\left(Y_{N}\right)$ & $\mathrm{d}\left(\mathrm{Y}_{\mathrm{P}}\right)$ & $\mathrm{d}\left(\mathrm{Y}_{\mathrm{K}}\right)$ \\
\hline & & & & & & & & $\mathrm{tha}^{-1}$ & & & & & - tha ${ }^{-1}$ & \\
\hline 000 & -51 & 124 & 51 & 38 & -104 & -60 & 71 & 20,00 & $\mathrm{~V}^{+}$ & $\mathrm{V}^{-}$ & $\mathrm{V}^{-}$ & 6,53 & 0,42 & 1,25 \\
\hline 001 & -50 & 116 & 1 & 69 & -107 & -29 & 62 & 18,75 & $\mathrm{~V}^{+}$ & $\mathrm{V}^{-}$ & $\mathrm{V}^{-}$ & 6,27 & 0,17 & 0,17 \\
\hline 002 & -53 & 94 & -20 & 81 & -87 & -15 & 58 & 18,58 & $\mathrm{~V}^{+}$ & $F^{-}$ & $\mathrm{V}^{+}$ & 6,11 & $-0,17$ & 0,92 \\
\hline 003 & -69 & 87 & -4 & 73 & -63 & -24 & 53 & 19,50 & $\mathrm{~V}^{+}$ & $\mathrm{F}^{-}$ & - & 5,86 & $-0,42$ & 0,00 \\
\hline 010 & -69 & 121 & 33 & 37 & -80 & -42 & 64 & 19,58 & $\mathrm{~V}^{+}$ & $\mathrm{F}^{*}$ & $\mathrm{~V}^{-}$ & 6,20 & $-0,26$ & 1,00 \\
\hline 011 & -64 & 138 & -8 & 67 & -96 & -37 & 68 & 18,58 & $\mathrm{~V}^{+}$ & $\mathrm{F}^{-}$ & $\mathrm{V}^{+}$ & 5,94 & $-0,59$ & 0,17 \\
\hline 012 & -67 & 118 & -15 & 81 & -92 & -25 & 66 & 18,75 & $\mathrm{~V}^{+}$ & $\mathrm{F}^{-}$ & $\mathrm{V}^{+}$ & 5,69 & $-0,83$ & 1,17 \\
\hline 013 & -78 & 82 & 17 & 78 & -68 & -31 & 59 & & $\mathrm{~V}^{+}$ & $\mathrm{F}^{-}$ & - & 52 & $-1,17$ & 0,00 \\
\hline 020 & -97 & 137 & 26 & 18 & -48 & -35 & 60 & 19,84 & $\mathrm{~V}^{+}$ & $\mathrm{F}^{-}$ & $\mathrm{V}^{-}$ & 85 & $-1,00$ & 0,67 \\
\hline 021 & -88 & 131 & 2 & 54 & -73 & -26 & 62 & 19,17 & $\mathrm{~V}^{+}$ & $\mathrm{F}^{-}$ & $F^{-}$ & 5,52 & $-1,25$ & $-0,41$ \\
\hline 022 & -93 & 107 & 7 & 67 & -70 & -18 & 60 & 19,58 & $\mathrm{~V}^{+}$ & $F^{-}$ & $F^{-}$ & 5,28 & $-1,51$ & $-1,51$ \\
\hline 023 & -106 & 97 & 43 & 61 & -64 & -31 & 67 & 21,09 & $\mathrm{~V}^{+}$ & $\mathrm{F}^{-}$ & - & 5,02 & $-1,76$ & 0,00 \\
\hline 030 & -128 & 129 & 43 & -4 & -17 & -24 & 58 & 20,84 & $\mathrm{~V}^{+}$ & - & $\mathrm{V}^{-}$ & 5,44 & 0,00 & 0,42 \\
\hline 031 & -126 & 142 & 30 & 22 & -42 & -26 & 65 & 20,42 & $\mathrm{~V}^{+}$ & - & $\mathrm{F}^{-}$ & 5,19 & 0,00 & $-0,67$ \\
\hline 032 & -124 & 123 & 49 & 39 & -50 & -37 & 70 & & $\mathrm{~V}^{+}$ & - & $\mathrm{F}^{-}$ & 94 & & $-1,76$ \\
\hline 033 & -135 & 112 & 85 & 34 & -46 & -50 & 77 & 22,85 & $\mathrm{~V}^{+}$ & - & - & 4,68 & 0,00 & 0,00 \\
\hline 100 & -12 & 82 & 53 & 55 & -94 & -84 & 63 & 26,53 & $\mathrm{~V}^{+}$ & $\mathrm{V}^{-}$ & $\mathrm{V}^{-}$ & 3,93 & 0,75 & 1,51 \\
\hline 101 & -6 & 101 & 11 & 76 & -97 & -85 & 63 & 25,02 & $\mathrm{~V}^{+}$ & $\mathrm{V}^{-}$ & $\mathrm{V}^{\cdot}$ & 3,69 & 0,50 & 0,33 \\
\hline 102 & -15 & 104 & -4 & 75 & -80 & -81 & 60 & 24,69 & $\mathrm{~V}^{+}$ & $\mathrm{V}^{-}$ & $\mathrm{V}^{+}$ & 3,43 & 0,25 & 0,67 \\
\hline 103 & -20 & 66 & 26 & 67 & -55 & -84 & 53 & 25,36 & $\mathrm{~V}^{+}$ & $F^{-}$ & - & 3,18 & $-0,08$ & 0,00 \\
\hline 110 & -18 & 84 & 23 & 61 & -67 & -82 & 56 & 25,78 & $\mathrm{~V}^{+}$ & $\mathrm{V}^{-}$ & $\mathrm{V}^{-}$ & 3,51 & 0,09 & 1,26 \\
\hline 111 & -18 & 97 & -14 & 82 & -86 & -62 & 60 & 24,52 & $\mathrm{~V}^{+}$ & $\mathrm{F}^{-}$ & $\mathrm{F}^{+}$ & 3,27 & $-0,17$ & $-0,08$ \\
\hline 112 & -17 & 103 & -11 & 87 & -82 & -80 & 63 & 24,44 & $\mathrm{~V}^{+}$ & $\mathrm{F}^{-}$ & $\mathrm{V}^{+}$ & 3,01 & $-0,42$ & 1,00 \\
\hline 113 & -39 & 87 & 23 & 68 & -65 & -76 & 60 & 25,44 & $\mathrm{~V}^{+}$ & $\mathrm{F}^{-}$ & - & 2,76 & $-0,67$ & 0,00 \\
\hline 120 & -40 & 80 & 9 & 56 & -44 & -61 & 48 & 25,69 & $\mathrm{~V}^{+}$ & $\mathrm{F}^{-}$ & $\mathrm{V}^{-}$ & 3,10 & $-0,59$ & 1,00 \\
\hline 121 & -33 & 95 & -13 & 76 & -60 & -65 & 57 & 24,69 & $\mathrm{~V}^{+}$ & $\mathrm{F}^{-}$ & $\mathrm{V}^{+}$ & 2,93 & $-0,92$ & 0,17 \\
\hline 122 & -38 & 95 & -1 & 81 & -73 & -65 & 59 & 24,86 & $\mathrm{~V}^{+}$ & $\mathrm{F}^{-}$ & $\mathrm{V}^{+}$ & 2,67 & $-1,17$ & 1,25 \\
\hline 123 & -49 & 83 & 45 & 69 & -68 & -80 & 66 & 26,11 & $\mathrm{~V}^{+}$ & $\mathrm{F}^{-}$ & - & 2,43 & $-1,42$ & 0,00 \\
\hline 130 & -70 & 104 & 14 & 35 & -12 & -72 & 51 & 26,28 & $\mathrm{~V}^{+}$ & - & $\mathrm{V}^{-}$ & 2,76 & 0,00 & 0,67 \\
\hline 131 & -68 & 113 & 0 & 54 & -41 & -59 & 56 & 25,61 & $\mathrm{~V}^{+}$ & - & $F^{-}$ & 2,51 & 0,00 & $-0,42$ \\
\hline 132 & -68 & 114 & 29 & 59 & -54 & -81 & 68 & 26,03 & $\mathrm{~V}^{+}$ & - & $\mathrm{F}^{-}$ & 2,34 & 0,00 & $-1,50$ \\
\hline 133 & -74 & 102 & 80 & 49 & -61 & -95 & 77 & 27,53 & $\mathrm{~V}^{+}$ & - & - & 2,10 & 0,00 & 0,00 \\
\hline
\end{tabular}


Apêndice 40. Índices DRIS, calculados pelo procedimento proposto por Jones (1981), diagnose para a correção de N, P e K, resposta líquida da produtividade, diagnose para o nutriente deficiente e a resposta da produtividade, para os dados, dos tratamentos com as doses 2 e 3 de $\mathrm{N}$ em todas as combinações de doses de $\mathrm{P}$ e $\mathrm{K}$, do ensaio de adubação $\mathrm{N}, \mathrm{P}$ e $\mathrm{K}$ conduzido em Monte Azul /SP.

\begin{tabular}{|c|c|c|c|c|c|c|c|c|c|c|c|c|c|c|}
\hline \multirow[b]{2}{*}{ Trat. } & \multicolumn{6}{|c|}{ Indices DRIS } & \multirow[b]{2}{*}{$\mathrm{BN}$} & \multirow[b]{2}{*}{ Prod. } & \multicolumn{3}{|c|}{ Diagnose } & \multicolumn{3}{|c|}{ Resposta } \\
\hline & $\mathrm{N}$ & $P$ & $\mathrm{~K}$ & $\mathrm{Ca}$ & $\mathrm{Mg}$ & $\bar{S}$ & & & $\mathrm{~N}$ & $P$ & $\mathrm{~K}$ & $\mathrm{~d}\left(\mathrm{Y}_{\mathrm{N}}\right)$ & $d\left(Y_{P}\right)$ & $\mathrm{d}\left(\mathrm{Y}_{\mathrm{K}}\right)$ \\
\hline & & & & & & & & tha $\mathrm{h}^{-1}$ & & & & & tha- & \\
\hline 200 & 0 & 54 & 53 & 67 & -76 & -98 & 58 & 30,46 & $F^{-}$ & $\mathrm{V}^{-}$ & $\mathrm{V}^{-}$ & $-1,26$ & 1,17 & 1,75 \\
\hline 201 & 0 & 69 & 14 & 78 & -81 & -81 & 54 & 28,71 & $\mathrm{~F}^{-}$ & $\mathrm{V}^{-}$ & $\mathrm{V}^{-}$ & $-1,00$ & 0,92 & 0,59 \\
\hline 202 & -4 & 70 & 8 & 71 & -65 & -79 & 50 & 28,12 & $\mathrm{~V}^{+}$ & $\mathrm{V}^{-}$ & $\mathrm{F}^{-}$ & 0,75 & 0,67 & $-0,42$ \\
\hline 203 & -13 & 59 & 43 & 49 & -45 & -93 & 50 & 28,54 & $\mathrm{~V}^{+}$ & $\mathrm{V}^{-}$ & - & 0,58 & 0,34 & 0,00 \\
\hline 210 & 9 & 25 & 13 & 91 & -57 & -81 & 46 & 29,29 & $\mathrm{~F}^{*}$ & $\mathrm{~V}^{-}$ & $\mathrm{V}^{-}$ & $-0,84$ & 0,50 & 1,50 \\
\hline 211 & 3 & 71 & -25 & 92 & -66 & .75 & 55 & 27,79 & $\mathrm{~F}^{-}$ & $\mathrm{V}^{-}$ & $\mathrm{F}^{+}$ & $-0,67$ & 0,17 & $-0,34$ \\
\hline 212 & -1 & 71 & -14 & 87 & -66 & -77 & 53 & 27,45 & $\mathrm{~V}^{+}$ & $\mathrm{F}^{-}$ & $\mathrm{V}^{+}$ & 0,42 & $-0,08$ & 0,75 \\
\hline 213 & -14 & 57 & 35 & 65 & -49 & -95 & 53 & 28 & $\mathrm{~V}^{+}$ & $\mathrm{F}^{-}$ & - & 17 & 34 & 0,00 \\
\hline 220 & -15 & 57 & -23 & 82 & -36 & -66 & 47 & 28,79 & $\mathrm{~V}^{+}$ & $\mathrm{F}^{-}$ & $\mathrm{F}^{+}$ & 0,50 & $-0,25$ & $-1,17$ \\
\hline 221 & -6 & 72 & -37 & 96 & -52 & -73 & 56 & 27,62 & $\mathrm{~V}^{+}$ & $\mathrm{F}^{-}$ & $\mathrm{F}^{+}$ & 0,25 & $-0,50$ & $-0,09$ \\
\hline 222 & -12 & 69 & -15 & 90 & -55 & -78 & 53 & 27,53 & $\mathrm{~F}^{+}$ & $\mathrm{F}^{-}$ & $\mathrm{V}^{+}$ & 0,00 & $-0,84$ & 1,01 \\
\hline 223 & -20 & 53 & 45 & 71 & -51 & -98 & 56 & 28,54 & $\mathrm{~F}^{+}$ & $\mathrm{F}^{-}$ & - & $-0,17$ & $-1,09$ & 0,00 \\
\hline 230 & -36 & 57 & -28 & 72 & 1 & -65 & 43 & 29,04 & $\mathrm{~V}^{+}$ & - & $\mathrm{F}^{+}$ & 0,08 & 0,00 & $-0,92$ \\
\hline 231 & -31 & 65 & -32 & 84 & -30 & -55 & 50 & 28,12 & $\mathrm{~F}^{+}$ & - & $\mathrm{V}^{+}$ & $-0,08$ & 0 & 0,25 \\
\hline 232 & -29 & 65 & 8 & 83 & -44 & -83 & 52 & 28,37 & $\mathrm{~F}^{+}$ & - & $\mathrm{F}^{-}$ & $-0,42$ & 0,00 & $-1,26$ \\
\hline 233 & -49 & 75 & 66 & 57 & -59 & -90 & 66 & 29,63 & $F^{+}$ & - & - & $-0,59$ & 0,00 & 0,00 \\
\hline 300 & -18 & -39 & 51 & 80 & -36 & -38 & 44 & 31,72 & - & $\mathrm{F}^{+}$ & $\mathrm{V}^{-}$ & 0,00 & $-1,59$ & 2,01 \\
\hline 301 & -19 & 24 & 16 & 77 & -43 & -55 & 39 & 29,71 & - & $\mathrm{V}^{-}$ & $\mathrm{V}^{-}$ & 0,00 & 1,25 & 0,84 \\
\hline 302 & -26 & 60 & 17 & 59 & -45 & -65 & 45 & 28,87 & - & $\mathrm{V}^{-}$ & $\mathrm{F}^{-}$ & 0,00 & 1,00 & $-0,25$ \\
\hline 303 & -41 & 40 & 57 & 26 & -19 & -63 & 41 & 29 , & - & $\mathrm{V}^{*}$ & - & 0,00 & 0,75 & 0,00 \\
\hline 310 & -10 & -29 & -11 & 106 & -31 & -25 & 35 & 30 & - & $\mathrm{F}^{+}$ & $\mathrm{F}^{+}$ & 00 & $-0,84$ & $-1,67$ \\
\hline 311 & -8 & 30 & -38 & 101 & -39 & -45 & 44 & 28,46 & - & $\mathrm{V}^{-}$ & $\mathrm{F}^{+}$ & 0,00 & 0,59 & $-0,59$ \\
\hline 312 & -10 & 27 & -16 & 89 & -41 & -50 & 39 & 27,87 & - & $\mathrm{V}^{-}$ & $\mathrm{V}^{+}$ & 0,00 & 0,34 & 0,50 \\
\hline 313 & -34 & 42 & 36 & 51 & -32 & -61 & 43 & 28,37 & - & $\mathrm{V}^{-}$ & - & 0,00 & 0,00 & 0,00 \\
\hline 320 & -13 & -24 & -59 & 113 & -1 & -15 & 38 & 29,29 & - & $\mathrm{F}^{+}$ & $\mathrm{F}^{+}$ & 0,00 & $-0,17$ & $-1,42$ \\
\hline 321 & -7 & 34 & -69 & 113 & -36 & -36 & 49 & 27,87 & - & $\mathrm{F}^{-}$ & $\mathrm{F}^{+}$ & 0,00 & $-0,17$ & $-0,34$ \\
\hline 322 & -13 & 28 & -30 & 101 & -40 & -46 & 43 & 27,53 & - & $\mathrm{F}^{-}$ & $\mathrm{V}^{+}$ & 0,00 & $-0,42$ & 0,84 \\
\hline 323 & -35 & 42 & 33 & 64 & -45 & -60 & 47 & 28,37 & - & $\mathrm{F}^{-}$ & - & 0,00 & $-0,67$ & 0,00 \\
\hline 330 & -22 & -21 & -87 & 111 & 28 & -8 & 46 & 29,12 & - & - & $\mathrm{F}^{+}$ & 0,00 & 0,00 & $-1,08$ \\
\hline 331 & -26 & 28 & -80 & 106 & -12 & -15 & 45 & 28,04 & - & - & $\mathrm{F}^{+}$ & 0,00 & 0,00 & $-0,09$ \\
\hline 332 & -25 & 26 & -20 & 98 & -29 & -50 & 41 & 27,95 & - & - & $\mathrm{V}^{+}$ & 0,00 & 0,00 & 1,09 \\
\hline 333 & -47 & 36 & 51 & 61 & -36 & -65 & 49 & 29,04 & - & - & - & 0,00 & 0,00 & 0,00 \\
\hline
\end{tabular}


Apêndice 41. Índices DRIS, calculados pelo procedimento proposto por Jones (1981), diagnose para a correção de $\mathrm{N}, \mathrm{P}$ e $\mathrm{K}$, resposta líquida da produtividade, diagnose para o nutriente deficiente e a resposta da produtividade, para os dados, dos tratamentos com as doses 0 e 1 de $\mathrm{N}$ em todas as combinações de doses de $\mathrm{P}$ e $\mathrm{K}$, do ensaio de adubação $\mathrm{N}, \mathrm{P}$ e $\mathrm{K}$ conduzido em Olímpia/SP.

\begin{tabular}{|c|c|c|c|c|c|c|c|c|c|c|c|c|c|}
\hline \multirow[b]{2}{*}{ Trat. } & \multicolumn{5}{|c|}{ Indices DRIS } & \multirow[b]{2}{*}{$\mathrm{BN}$} & \multirow[b]{2}{*}{ Prod. } & \multicolumn{3}{|c|}{ Diagnose } & \multicolumn{3}{|c|}{ Resposta } \\
\hline & $\mathrm{N}$ & $\mathbf{P}$ & $\mathrm{K} \mathrm{Ca}$ & $\mathrm{Mg}$ & $\mathrm{S}$ & & & $\mathrm{N}$ & $\mathrm{p}$ & $\mathrm{K}$ & $\mathrm{d}\left(\mathrm{Y}_{\mathrm{N}}\right)$ & $d\left(Y_{P}\right)$ & $\mathrm{d}\left(\mathrm{Y}_{\mathrm{K}}\right)$ \\
\hline & & & & & & & $\mathrm{tha}^{-1}$ & & & & & tha ${ }^{-1}$ & \\
\hline 000 & 74 & 60 & $-20-142$ & 64 & -37 & 66 & 26,86 & $\mathrm{~V}^{-}$ & $\mathrm{V}^{-}$ & $\mathrm{F}^{+}$ & 0,25 & 0,77 & $-0,77$ \\
\hline 001 & 41 & 44 & $-54-109$ & 84 & -6 & 56 & 26,09 & $\mathrm{~V}^{-}$ & $\mathrm{V}^{-}$ & $\mathrm{F}^{+}$ & 0,08 & 0,51 & $-0,59$ \\
\hline 002 & 10 & 34 & $-69-104$ & 98 & 31 & 58 & 25,50 & $\mathrm{~V}^{*}$ & $\mathrm{~V}^{-}$ & $\mathrm{F}^{+}$ & 0,00 & 0,25 & $-0,51$ \\
\hline 003 & -10 & 38 & $-64-113$ & 111 & 38 & 62 & 24,99 & $\mathrm{~V}^{+}$ & $\mathrm{V}^{-}$ & - & 0,17 & 0,00 & 0,00 \\
\hline 010 & 40 & 60 & $-37-129$ & 76 & -10 & 59 & 26,09 & $\mathrm{~V}^{-}$ & $\mathrm{F}^{-}$ & $\mathrm{F}^{+}$ & 0,84 & $-0,43$ & $-0,51$ \\
\hline 011 & 6 & 36 & $-66-110$ & 100 & 34 & 59 & 25,58 & $\mathrm{~V}^{-}$ & $\mathrm{F}^{-}$ & $\mathrm{F}^{+}$ & 0,67 & $-0,77$ & $-0,33$ \\
\hline 012 & -12 & 28 & $-70-108$ & 114 & 48 & 63 & 25,25 & $\mathrm{~F}^{+}$ & $\mathrm{F}^{-}$ & $\mathrm{F}^{+}$ & $-0,52$ & $-1,02$ & $-0,26$ \\
\hline 013 & -23 & 30 & $-49-125$ & 116 & 51 & 66 & 24,99 & $\mathrm{~F}^{+}$ & $F^{-}$ & - & $-0,26$ & $-1,36$ & 0,00 \\
\hline 020 & 28 & 36 & $-40-123$ & 89 & 11 & 55 & 26,52 & $\mathrm{~V}^{-}$ & $\mathrm{F}^{-}$ & $\mathrm{F}^{+}$ & 1,36 & $-1,79$ & $-0,17$ \\
\hline 021 & 1 & 48 & $-63-110$ & 99 & 25 & 58 & 26,35 & $\mathrm{~V}^{-}$ & $F^{-}$ & $\mathrm{F}^{+}$ & 1,19 & $-2,04$ & $-0,08$ \\
\hline 022 & -17 & 33 & $-60-119$ & 109 & 54 & 65 & 26,27 & $\mathrm{~F}^{+}$ & $\mathrm{F}^{-}$ & $\mathrm{V}^{+}$ & $-1,02$ & $-2,37$ & 0,08 \\
\hline 023 & -14 & 39 & $-23-140$ & 101 & 37 & 59 & 26,35 & $\mathrm{~F}^{+}$ & $\mathrm{F}^{-}$ & - & $-0,85$ & $-2,63$ & 0,00 \\
\hline 030 & 28 & 57 & $-46-125$ & 75 & 12 & 57 & 28,31 & $\mathrm{~V}^{*}$ & - & $\mathrm{V}^{+}$ & 1,88 & 0,00 & 0,08 \\
\hline 031 & 15 & 32 & $-53-115$ & 89 & 32 & 56 & 28,39 & $\mathrm{~V}^{-}$ & - & $\mathrm{V}^{+}$ & 1,70 & 0,00 & 0,25 \\
\hline 032 & 10 & 19 & $-33-127$ & 89 & 41 & 53 & 28,64 & $\mathrm{~V}^{*}$ & - & $\mathrm{V}^{+}$ & 1,53 & 0,00 & 0,34 \\
\hline 033 & 19 & 22 & $9-160$ & 88 & 21 & 53 & 28,98 & $\mathrm{~V}^{-}$ & - & - & 1,36 & 0,00 & 0,00 \\
\hline 100 & 55 & 42 & $-15-125$ & 78 & -35 & 58 & 26,61 & $\mathrm{~F}^{-}$ & $\mathrm{V}^{-}$ & $\mathrm{F}^{+}$ & $-0,76$ & 1,36 & $-0,60$ \\
\hline 101 & 33 & 29 & $-49-101$ & 90 & -2 & 51 & 26,01 & $F^{*}$ & $\mathrm{~V}^{*}$ & $\mathrm{~F}^{+}$ & $-1,02$ & 1,10 & $-0,51$ \\
\hline 102 & 18 & 24 & $\begin{array}{ll}-62 & -97\end{array}$ & 99 & 18 & 53 & 25,50 & $F^{-}$ & $\mathrm{V}^{-}$ & $\mathrm{F}^{+}$ & $-1,19$ & 0,77 & $-0,34$ \\
\hline 103 & 12 & 32 & $-53-112$ & 96 & 27 & 55 & 25,16 & $\mathrm{~F}^{-}$ & $\mathrm{V}^{-}$ & - & $-1,36$ & 0,43 & 0,00 \\
\hline 110 & 19 & 36 & $-45-115$ & 97 & 9 & 54 & 25,25 & $\mathrm{~F}^{-}$ & $\mathrm{V}^{-}$ & $\mathrm{F}^{+}$ & $-0,25$ & 0,09 & $-0,34$ \\
\hline 111 & 8 & 25 & $-66-96$ & 108 & 20 & 54 & 24,91 & $\mathrm{~F}^{-}$ & $\mathrm{F}^{-}$ & $\mathrm{F}^{+}$ & $-0,51$ & $-0,25$ & $-0,18$ \\
\hline 112 & 1 & 20 & $\begin{array}{ll}-66 & -98\end{array}$ & 106 & 36 & 55 & 24,73 & $\mathrm{~F}^{-}$ & $\mathrm{F}^{-}$ & $\mathrm{F}^{+}$ & $-0,69$ & $-0,52$ & 0,00 \\
\hline 113 & 5 & 29 & $-43-116$ & 102 & 24 & 53 & 24,73 & $\mathrm{~F}^{-}$ & $F^{-}$ & - & $-0,77$ & $-0,77$ & 0,00 \\
\hline 120 & 16 & 15 & $-58-98$ & 113 & 13 & 52 & 25,16 & $\mathrm{~V}^{-}$ & $\mathbf{F}^{-}$ & $\mathrm{F}^{+}$ & 0,25 & $-1,27$ & 0,00 \\
\hline 121 & 2 & 32 & $\begin{array}{ll}-76 & -94\end{array}$ & 107 & 29 & 57 & 25,16 & $\mathrm{~V}^{-}$ & $\mathrm{F}^{-}$ & $\mathrm{V}^{+}$ & 0,08 & $-1,53$ & 0,09 \\
\hline 122 & 4 & 27 & $-63-102$ & 110 & 23 & 55 & 25,25 & $F^{*}$ & $\mathrm{~F}^{-}$ & $\mathrm{V}^{+}$ & $-0,17$ & $-1,86$ & 0,25 \\
\hline 123 & 18 & 36 & $-32-128$ & 96 & 9 & 53 & 25,50 & $\mathrm{~F}^{-}$ & $F^{-}$ & - & $-0,34$ & $-2,12$ & 0,00 \\
\hline 130 & 22 & 35 & $\begin{array}{ll}-71 & -98\end{array}$ & 99 & 12 & 56 & 26,43 & $\mathrm{~V}^{-}$ & - & $\mathrm{V}^{+}$ & 0,76 & 0,00 & 0,26 \\
\hline 131 & 23 & 18 & $\begin{array}{ll}-74 & -91\end{array}$ & 107 & 17 & 55 & 26,69 & $\mathrm{~V}^{-}$ & - & $\mathrm{V}^{+}$ & 0,60 & 0,00 & 0,42 \\
\hline 132 & 34 & 13 & $-51-105$ & 101 & 9 & 52 & 27,11 & $\mathrm{~V}^{-}$ & - & $\mathrm{V}^{+}$ & 0,42 & 0,00 & 0,51 \\
\hline 133 & 55 & 19 & $-5-141$ & 83 & -11 & 52 & 27,62 & $\mathrm{~V}^{-}$ & - & - & 0,17 & 0,00 & 0,00 \\
\hline
\end{tabular}


Apêndice 42. Índices DRIS, calculados pelo procedimento proposto por Jones (1981), diagnose para a correção de $\mathrm{N}, \mathrm{P}$ e K, resposta líquida da produtividade, diagnose para o nutriente deficiente e a resposta da produtividade, para os dados, dos tratamentos com as doses 2 e 3 de $\mathrm{N}$ em todas as combinações de doses de $\mathrm{P}$ e $\mathrm{K}$, do ensaio de adubação $\mathrm{N}, \mathrm{P}$ e $\mathrm{K}$ conduzido em Olímpia/SP.

\begin{tabular}{|c|c|c|c|c|c|c|c|c|c|c|c|c|c|c|}
\hline \multirow[b]{2}{*}{ Trat. } & \multicolumn{6}{|c|}{ Índices DRIS } & \multirow[b]{2}{*}{ BN } & \multirow[b]{2}{*}{ Prod. } & \multicolumn{3}{|c|}{ Diagnose } & \multicolumn{3}{|c|}{ Resposta } \\
\hline & $\overline{\mathrm{N}}$ & $P$ & $\mathrm{~K}$ & $\mathrm{Ca}$ & $\mathrm{Mg}$ & $\bar{S}$ & & & $\mathrm{~N}$ & $P$ & $\mathrm{~K}$ & $\overline{d\left(Y_{N}\right)}$ & $\mathrm{d}\left(\mathrm{Y}_{\mathrm{P}}\right)$ & $\mathrm{d}\left(\mathrm{Y}_{\mathrm{K}}\right)$ \\
\hline & & & & & & & & $\mathrm{tha}^{-1}$ & & & & & tha & \\
\hline 200 & 40 & 35 & -30 & -122 & 93 & -17 & 56 & 27,37 & $\mathrm{~F}^{-}$ & $\mathrm{V}^{-}$ & $\mathrm{F}^{+}$ & $-1,96$ & 1,87 & $-0,34$ \\
\hline 201 & 31 & 24 & -67 & -104 & 98 & 18 & 57 & 27,03 & $\mathrm{~F}^{-}$ & $\mathrm{V}^{-}$ & $\mathrm{F}^{+}$ & $-2,13$ & 1,61 & $-0,34$ \\
\hline 202 & 33 & 28 & -76 & -101 & 93 & 23 & 59 & 26,69 & $\mathrm{~F}^{-}$ & $\mathrm{V}^{-}$ & $\mathrm{F}^{+}$ & $-2,29$ & 1,27 & $-0,17$ \\
\hline 203 & 40 & 42 & -61 & -120 & 84 & 16 & 61 & 26,52 & $\mathrm{~F}^{-}$ & $\mathrm{V}^{-}$ & - & $-2,55$ & 1,02 & 0,00 \\
\hline 210 & 16 & 34 & -66 & -106 & 114 & 9 & 58 & 25,50 & $\mathrm{~F}^{-}$ & $\mathrm{V}^{*}$ & $\mathrm{~F}^{+}$ & $-1,44$ & 0,59 & $-0,08$ \\
\hline 211 & 14 & 25 & -89 & -91 & 118 & 22 & 60 & 25,42 & $\mathrm{~F}^{-}$ & $\mathrm{V}^{-}$ & $\mathrm{F}^{+}$ & $-1,61$ & 0,34 & 0,00 \\
\hline 212 & 24 & 26 & -87 & -96 & 110 & 23 & 61 & 25,42 & $\mathrm{~F}^{-}$ & $\mathrm{V}^{-}$ & $\mathrm{V}^{+}$ & $-1,78$ & 0,00 & 0,08 \\
\hline 213 & 48 & 4 & -55 & -115 & 95 & 22 & 57 & 25,50 & $\mathrm{~F}^{-}$ & $\mathrm{F}^{-}$ & - & $-1,95$ & $-0,34$ & 0,00 \\
\hline 220 & 13 & 7 & -94 & -88 & 129 & 32 & 61 & 24,91 & $F^{-}$ & $\mathrm{F}^{-}$ & $\mathrm{V}^{+}$ & $-0,93$ & $-0,76$ & 0,17 \\
\hline 221 & 23 & -4 & -101 & -82 & 122 & 42 & 62 & 25,08 & $\mathrm{~F}^{-}$ & $\mathrm{V}^{+}$ & $\mathrm{V}^{+}$ & $-1,10$ & 1,01 & 0,34 \\
\hline 222 & 36 & -4 & -91 & -92 & 112 & 40 & 63 & 25,42 & $F^{-}$ & $\mathrm{V}^{+}$ & $\mathrm{V}^{+}$ & $-1,19$ & 1,27 & 0,42 \\
\hline 223 & 62 & 10 & -50 & -124 & 98 & 5 & 58 & 25,84 & $\mathrm{~F}^{-}$ & $\mathbf{F}^{-}$ & - & $-1,45$ & $-1,61$ & 0,00 \\
\hline 230 & 26 & 31. & -117 & -80 & 128 & 13 & 66 & 25,67 & $F^{*}$ & - & $\mathrm{V}^{+}$ & $-0,34$ & 0,00 & 0,42 \\
\hline 231 & 41 & 17. & -116 & -80 & 118 & 20 & 65 & 26,09 & $\mathrm{~F}^{-}$ & - & $\mathrm{V}^{+}$ & $-0,60$ & 0,00 & 0,60 \\
\hline 232 & 68 & 19 & -89 & -96 & 105 & -7 & 64 & 26,69 & $\mathrm{~F}^{-}$ & - & $\mathrm{V}^{+}$ & $-0,76$ & 0,00 & 0,76 \\
\hline 233 & 105 & 32 & -37 & -135 & 81 & -47 & 73 & 27,45 & $\mathrm{~F}^{-}$ & - & - & $-0,86$ & 0,00 & 0,00 \\
\hline 300 & 44 & 7 & -61 & -126 & 107 & 28 & 62 & 29,33 & - & $\mathrm{V}^{-}$ & $\mathrm{F}^{+}$ & 0,00 & 2,39 & $-0,17$ \\
\hline 301 & 49 & 1 & -95 & 109 & 107 & 47 & 68 & 29,16 & - & $\mathrm{V}^{-}$ & $\mathrm{F}^{+}$ & 0,00 & 2,13 & $-0,18$ \\
\hline 302 & 68 & 13 & -104 & -110 & 96 & 37 & 71 & 28,98 & - & $\mathrm{V}^{*}$ & $\mathrm{~V}^{+}$ & 0,00 & 1,78 & 0,09 \\
\hline 303 & 94 & 34 & -87 & -133 & 80 & 11 & 73 & 29,07 & - & $\mathrm{V}^{\circ}$ & - & 0,00 & 1,62 & 0,00 \\
\hline 310 & 20 & & -114 & -104 & 139 & 55 & 73 & 26,94 & - & $\mathrm{V}^{-}$ & $\mathrm{V}^{+}$ & 0,00 & 1,10 & 0,09 \\
\hline 311 & 40 & & -132 & -92 & 129 & 53 & 75 & 27,03 & - & $\mathrm{V}^{-}$ & $\mathrm{V}^{+}$ & 0,00 & 0,85 & 0,17 \\
\hline 312 & 62 & 10 & -123 & 100 & 114 & 37 & 74 & 27,20 & - & $\mathrm{V}^{-}$ & $\mathrm{V}^{+}$ & 0,00 & 0,59 & 0,25 \\
\hline 313 & 97 & 31 & -96 & -129 & 87 & 8 & 75 & 27,45 & - & $\mathrm{V}^{-}$ & - & 0,00 & 0,16 & 0,00 \\
\hline 320 & 23 & 16 & -156 & -86 & 152 & 51 & 81 & 25,84 & - & $F^{-}$ & $\mathrm{V}^{+}$ & 0,00 & $-0,17$ & 0,34 \\
\hline 321 & 47 & 12 & -161 & -81 & 138 & 45 & 81 & 26,18 & - & $F^{-}$ & $\mathrm{V}^{+}$ & 0,00 & $-0,51$ & 0,43 \\
\hline 322 & 77 & 18 & -143 & -96 & 120 & 24 & 80 & 26,61 & - & $\mathrm{F}^{-}$ & $\mathrm{V}^{+}$ & 0,00 & $-0,84$ & 0,68 \\
\hline 323 & 127 & -1 & -96 & -126 & 96 & 0 & 74 & 27,29 & - & $\mathrm{V}^{+}$ & - & 0,00 & 1,02 & 0,00 \\
\hline 330 & 54 & & -201 & -67 & 162 & 49 & 89 & 26,01 & - & - & $\mathrm{V}^{+}$ & 0,00 & 0,00 & 0,68 \\
\hline 331 & 85 & -5 & -189 & -70 & 143 & 36 & 88 & 26,69 & - & - & $\mathrm{V}^{+}$ & 0,00 & 0,00 & 0,76 \\
\hline 332 & 124 & 1 & -152 & -91 & 112 & 8 & 81 & 27,45 & - & - & $\mathrm{V}^{+}$ & 0,00 & 0,00 & 0,86 \\
\hline 333 & 181 & 23 & -89 & -135 & 79 & -59 & 94 & 28,31 & - & - & - & 0,00 & 0,00 & 0,00 \\
\hline
\end{tabular}


Apêndice 43. Índices DRIS, calculados pelo procedimento proposto por Jones (1981), diagnose para a correção de N, P e K, resposta líquida da produtividade, diagnose para o nutriente deficiente e a resposta da produtividade, para os dados, dos tratamentos com as doses 0 e 1 de $\mathrm{N}$ em todas as combinações de doses de $\mathrm{P}$ e $\mathrm{K}$, do ensaio de adubação $\mathrm{N}, \mathrm{P}$ e $\mathrm{K}$ conduzido em Pirassununga/SP.

\begin{tabular}{|c|c|c|c|c|c|c|c|c|c|c|c|c|c|c|}
\hline \multirow[b]{2}{*}{ Trat. } & \multicolumn{6}{|c|}{ Índices DRIS } & \multirow[b]{2}{*}{$\mathrm{BN}$} & \multirow[b]{2}{*}{ Prod. } & \multicolumn{3}{|c|}{ Diagnose } & \multicolumn{3}{|c|}{ Resposta } \\
\hline & $\mathrm{N}$ & $P$ & $\mathrm{~K}$ & $\mathrm{Ca}$ & $\mathrm{Mg}$ & $\mathbf{S}$ & & & $\mathrm{N}$ & $P$ & $\mathrm{~K}$ & $\mathrm{~d}\left(\mathrm{Y}_{\mathrm{N}}\right)$ & $\mathrm{d}\left(\mathrm{Y}_{\mathrm{P}}\right)$ & $\mathrm{d}\left(\mathrm{Y}_{\mathrm{K}}\right)$ \\
\hline & & & & & & & & $\mathrm{tha}^{-1}$ & & & & & 10 & \\
\hline 000 & -81 & 13 & -94 & 89 & 120 & -48 & 74 & 35,50 & $\mathrm{~V}^{+}$ & $\mathrm{V}^{-}$ & $\mathrm{V}^{+}$ & 1,76 & 2,18 & 0,95 \\
\hline 001 & -76 & 14 & -17 & 79 & 80 & -81 & 58 & 36,45 & $\mathrm{~V}^{+}$ & $\mathrm{V}^{-}$ & $\mathrm{F}^{+}$ & 1,49 & 1,23 & $-0,55$ \\
\hline 002 & -79 & 10 & 29 & 73 & 55 & -88 & 56 & 35,90 & $\mathrm{~V}^{+}$ & $\mathrm{V}^{-}$ & $\mathrm{V}^{-}$ & 1,36 & 0,27 & 1,90 \\
\hline 003 & -86 & 47 & 53 & 68 & 17 & -98 & 62 & 34,00 & $\mathrm{~V}^{+}$ & $\mathrm{F}^{-}$ & - & 1,09 & $-0,82$ & 0,00 \\
\hline 010 & -60 & 21 & -90 & 95 & 101 & -67 & 72 & 33,32 & $\mathrm{~V}^{+}$ & $\mathrm{V}^{-}$ & $\mathrm{V}^{+}$ & 2,58 & 3,13 & 1,90 \\
\hline 011 & -76 & 16 & -15 & 83 & 71 & -79 & 57 & 35,22 & $\mathrm{~V}^{+}$ & $\mathrm{V}^{-}$ & $\mathrm{V}^{+}$ & 2,45 & 2,17 & 0,41 \\
\hline 012 & -84 & 13 & 35 & 77 & 46 & -85 & 57 & 35,63 & $\mathrm{~V}^{+}$ & $\mathrm{V}^{-}$ & $\mathrm{V}^{-}$ & 2,31 & 0,95 & 0,81 \\
\hline 013 & -100 & 49 & 55 & 72 & 19 & -94 & 65 & 34,82 & $\mathrm{~V}^{+}$ & $\mathrm{V}^{-}$ & - & 2,04 & 0,00 & 0,00 \\
\hline 020 & -42 & 57 & -97 & 77 & 83 & -79 & 73 & 30,19 & $\mathrm{~V}^{+}$ & $\mathrm{V}^{-}$ & $\mathrm{V}^{+}$ & 3,54 & 3,94 & 2,86 \\
\hline 021 & -58 & 15 & -13 & 72 & 69 & -83 & 52 & 33,05 & $\mathrm{~V}^{+}$ & $\mathrm{V}^{-}$ & $\mathrm{V}^{+}$ & 3,40 & 2,86 & 1,63 \\
\hline 022 & -74 & 13 & 34 & 68 & 45 & -87 & 54 & 34,68 & $\mathrm{~V}^{+}$ & $\mathrm{V}^{-}$ & $\mathrm{F}^{-}$ & 3,13 & 1,90 & $-0,14$ \\
\hline 023 & -100 & 48 & 57 & 62 & 30 & -96 & 66 & 34,82 & $\mathrm{~V}^{+}$ & $\mathrm{V}^{-}$ & - & 2,99 & 0,96 & 0,00 \\
\hline 030 & -9 & 83. & 106 & 47 & 83 & -98 & 71 & 26,25 & $\mathrm{~V}^{+}$ & - & $\mathrm{V}^{+}$ & 4,35 & 0,00 & 3,94 \\
\hline 031 & -34 & 47 & -21 & 41 & 69. & -101 & 52 & 30,19 & $\mathrm{~V}^{+}$ & - & $\mathrm{V}^{+}$ & 4,22 & 0,00 & 2,59 \\
\hline 032 & -60 & 44 & 28 & 36 & 56. & -104 & 55 & 32,78 & $\mathrm{~V}^{+}$ & - & $\mathrm{F}^{-}$ & 4,08 & 0,00 & $-1,08$ \\
\hline 033 & -95 & 74 & 51 & 31 & 52 & -111 & 69 & 33,86 & $\mathrm{~V}^{+}$ & - & - & 3,95 & 0,00 & 0,00 \\
\hline 100 & -55 & $16-$ & 180 & 112 & 162 & -55 & 97 & 37,26 & $\mathrm{~V}^{+}$ & $\mathrm{V}^{-}$ & $\mathrm{V}^{+}$ & 0,28 & 1,36 & 0,68 \\
\hline 101 & -49 & -31 & -94 & 104 & 133 & -63 & 79 & 37,94 & $\mathrm{~V}^{+}$ & $\mathrm{F}^{+}$ & $\mathrm{F}^{+}$ & 0,14 & $-0,27$ & $-0,68$ \\
\hline 102 & -53 & 11 & -49 & 84 & 89 & -83 & 62 & 37,26 & $\mathrm{~F}^{+}$ & $\mathrm{F}^{-}$ & $\mathrm{F}^{+}$ & 0,00 & $-0,68$ & $-2,17$ \\
\hline 103 & -46 & 52 & -26 & 74 & 62. & -117 & 63 & 35,09 & $\mathrm{~F}^{+}$ & $F^{-}$ & - & $-0,14$ & $-1,77$ & 0,00 \\
\hline 110 & -38 & -24 & 170 & 125 & 149 & -43 & 92 & 35,90 & $\mathrm{~V}^{+}$ & $\mathrm{F}^{+}$ & $\mathrm{V}^{+}$ & 1,23 & $-2,17$ & 1,77 \\
\hline 111 & -47 & -28 & -85 & 109 & 113 & -62 & 74 & 37,67 & $\mathrm{~V}^{+}$ & $\mathrm{F}^{+}$ & $\mathrm{V}^{+}$ & 1,09 & $-1,22$ & 0,27 \\
\hline 112 & -54 & -32 & -37 & 97 & 96 & -70 & 64 & 37,94 & $\mathrm{~V}^{+}$ & $\mathrm{F}^{+}$ & $\mathrm{F}^{+}$ & 0,96 & $-0,13$ & $-1,08$ \\
\hline 113 & -58 & 18 & -13 & 87 & & -104 & 58 & 36,86 & $\mathrm{~V}^{+}$ & $F^{-}$ & - & 0,81 & $-0,95$ & 0,00 \\
\hline 120 & -16 & 24 & 171 & 111 & 131 & -79 & 89 & 33,73 & $\mathrm{~V}^{+}$ & $\mathrm{V}^{-}$ & $\mathrm{V}^{+}$ & 2,17 & 3,13 & 2,72 \\
\hline 121 & -35 & -30 & -83 & 101 & 111 & -65 & 71 & 36,45 & $\mathrm{~V}^{+}$ & $\mathrm{F}^{+}$ & $\mathrm{V}^{+}$ & 2,04 & $-2,04$ & 1,36 \\
\hline 122 & -49 & -32 & -33 & 90 & 96 & -72 & 62 & 37,81 & $\mathrm{~V}^{+}$ & $\mathrm{F}^{+}$ & $\mathrm{F}^{+}$ & 1,77 & $-0,95$ & 0,00 \\
\hline 123 & -62 & 16 & -8 & 80 & 79 & -105 & 58 & 37,81 & $\mathrm{~V}^{+}$ & $\mathrm{V}^{-}$ & - & 1,63 & 0,00 & 0,00 \\
\hline 130 & 15 & $55-$ & 181 & 82 & 129 & -99 & 94 & 30,60 & $F^{-}$ & - & $\mathrm{V}^{+}$ & $-3,13$ & 0,00 & 3,81 \\
\hline 131 & -16 & 9 & -91 & 72 & 110 & -84 & 64 & 34,41 & $\mathrm{~V}^{+}$ & - & $\mathrm{V}^{+}$ & 2,85 & 0,00 & 2,45 \\
\hline 132 & -39 & 6 & -40 & 60 & 104 & -91 & 57 & 36,86 & $\mathrm{~V}^{+}$ & - & $\mathrm{V}^{+}$ & 2,72 & 0,00 & 0,95 \\
\hline 133 & -67 & 42 & -18 & 48 & 97 & -101 & 62 & 37,81 & $\mathrm{~V}^{+}$ & - & - & 2,58 & 0,00 & 0,00 \\
\hline
\end{tabular}


Apêndice 44. Índices DRIS, calculados pelo procedimento proposto por Jones (1981), diagnose para a correção de N, P e K, resposta líquida da produtividade, diagnose para o nutriente deficiente e a resposta da produtividade, para os dados, dos tratamentos com as doses 2 e 3 de $\mathrm{N}$ em todas as combinações de doses de $\mathrm{P}$ e $\mathrm{K}$, do ensaio de adubação $\mathrm{N}, \mathrm{P}$ e $\mathrm{K}$ conduzido em Pirassununga /SP.

\begin{tabular}{|c|c|c|c|c|c|c|c|c|c|c|c|c|c|c|}
\hline \multirow[b]{2}{*}{ Trat. } & \multicolumn{6}{|c|}{ Índices DRIS } & \multirow[b]{2}{*}{$\mathrm{BN}$} & \multirow[b]{2}{*}{ Prod. } & \multicolumn{3}{|c|}{ Diagnose } & \multicolumn{3}{|c|}{ Resposta } \\
\hline & $\mathrm{N}$ & $\mathrm{P}$ & $\mathrm{K}$ & $\mathrm{Ca}$ & $\mathrm{Mg}$ & $\mathrm{S}$ & & & $\mathrm{N}$ & $\mathbf{P}$ & $\mathrm{K}$ & $\mathrm{d}\left(\mathrm{Y}_{\mathrm{N}}\right)$ & $\mathrm{d}\left(\mathrm{Y}_{\mathrm{P}}\right)$ & $\mathrm{d}\left(\mathrm{Y}_{\mathrm{K}}\right)$ \\
\hline & & & & & & & & $\mathrm{tha}^{-1}$ & & & & & $-\mathrm{tha}^{-1}$ & \\
\hline 200 & -39 & 15 & -229 & 119 & 180 & -47 & 105 & 37,54 & $\mathrm{~F}^{+}$ & $\mathrm{V}^{-}$ & $\mathrm{V}^{+}$ & $-0,96$ & 0,41 & 0,54 \\
\hline 201 & -25 & -31 & -144 & 106 & 149 & -56 & 85 & 38,08 & $\mathrm{~F}^{+}$ & $\mathrm{V}^{+}$ & $\mathrm{F}^{+}$ & $-1,22$ & 0,68 & $-0,82$ \\
\hline 202 & -26 & 12 & -102 & 81 & 114 & -78 & 69 & 37,26 & $\mathrm{~F}^{+}$ & $\mathrm{F}^{-}$ & $\mathrm{F}^{+}$ & $-1,36$ & $-1,64$ & $-2,31$ \\
\hline 203 & -11 & 56 & -87 & 66 & 88 & -111 & 70 & 34,95 & $\mathrm{~F}^{+}$ & $\mathrm{F}^{-}$ & - & $-1,49$ & $-2,72$ & 0,00 \\
\hline 210 & -27 & -24 & -218 & 134 & 168 & -34 & 101 & 37,13 & $\mathrm{~F}^{+}$ & $\mathrm{F}^{+}$ & $\mathrm{V}^{+}$ & 0,00 & $-1,23$ & 1,63 \\
\hline 211 & -34 & -28 & -134 & 112 & 140 & -55 & 84 & 38,76 & $\mathrm{~F}^{+}$ & $\mathrm{F}^{+}$ & $\mathrm{V}^{+}$ & $-0,27$ & $-0,27$ & 0,14 \\
\hline 212 & -23 & -23 & -82 & 100 & 114 & -86 & 71 & 38,90 & $\mathrm{~F}^{+}$ & $\mathrm{V}^{+}$ & $\mathrm{F}^{+}$ & $-0,41$ & 0,68 & $-1,23$ \\
\hline 213 & -25 & 21 & -70 & 79 & 95 & -100 & 65 & 37,67 & $\mathrm{~F}^{+}$ & $F^{-}$ & - & $-0,68$ & $-1,77$ & 0,00 \\
\hline 220 & -9 & 24. & -211 & 120 & 148 & -72 & 97 & 35,90 & $\mathrm{~V}^{+}$ & $\mathrm{V}^{-}$ & $\mathrm{V}^{+}$ & 0,82 & 2,17 & 2,59 \\
\hline 221 & -24 & -30 & -131 & 105 & 138 & -58 & 81 & 38,49 & $\mathrm{~V}^{+}$ & $\mathrm{F}^{+}$ & $\mathrm{V}^{+}$ & 0,68 & $-1,23$ & 1,09 \\
\hline 222 & -28 & -31 & -85 & 90 & 121 & -67 & 70 & 39,58 & $\mathrm{~V}^{+}$ & $\mathrm{F}^{+}$ & $\mathrm{F}^{+}$ & 0,54 & 0,00 & $-0,14$ \\
\hline 223 & -37 & 17 & -64 & 72 & 113 & -102 & 68 & 39,44 & $\mathrm{~V}^{+}$ & $F^{-}$ & - & 0,27 & $-0,95$ & 0,00 \\
\hline 230 & 14 & 54. & -213 & 94 & 144 & -92 & 102 & 33,73 & $\mathrm{~F}^{-}$ & - & $\mathrm{V}^{+}$ & $-1,77$ & 0,00 & 3,53 \\
\hline 231 & -10 & & -134 & 78 & 134 & -78 & 74 & 37,26 & $\mathrm{~V}^{+}$ & - & $\mathrm{V}^{+}$ & 1,64 & 0,00 & 2,32 \\
\hline 232 & -23 & 7 & -90 & 62 & 128 & -85 & 66 & 39,58 & $\mathrm{~V}^{+}$ & - & $\mathrm{V}^{+}$ & 1,36 & 0,00 & 0,81 \\
\hline 233 & -39 & 6 & -68 & 51 & 136 & -87 & 65 & 40,39 & $\mathrm{~V}^{+}$ & - & - & 1,23 & 0,00 & 0,00 \\
\hline 300 & -39 & 16 & -222 & 113 & 179 & -48 & 103 & 36,58 & - & $\mathrm{F}^{-}$ & $\mathrm{V}^{+}$ & 0,00 & $-0,55$ & 0,28 \\
\hline 301 & -23 & 15 & -155 & 87 & 143 & -67 & 82 & 36,86 & - & $\mathrm{F}^{-}$ & $\mathrm{F}^{+}$ & 0,00 & $-1,63$ & $-0,96$ \\
\hline 302 & -1 & 15 & -118 & 64 & 116 & -77 & 65 & 35,90 & - & $\mathrm{F}^{-}$ & $\mathrm{F}^{+}$ & 0,00 & $-2,59$ & $-2,44$ \\
\hline 303 & 22 & 58 & -113 & 42 & 101 & -110 & 74 & 33,46 & - & $\mathrm{F}^{-}$ & - & 0,00 & $-3,53$ & 0,00 \\
\hline 310 & -36 & 20 & -206 & 121 & 148 & -47 & 96 & 37,13 & - & $\mathrm{V}^{-}$ & $\mathrm{V}^{+}$ & 0,00 & 0,41 & 1,36 \\
\hline 311 & -20 & -24 & -138 & 104 & 131 & -53 & 78 & 38,49 & - & $\mathrm{V}^{+}$ & $\mathrm{F}^{+}$ & 0,00 & 0,68 & 0,00 \\
\hline 312 & -8 & -25 & -102 & 82 & 115 & -62 & 66 & 38,49 & - & $\mathrm{V}^{+}$ & $\mathrm{F}^{+}$ & 0,00 & 1,63 & $-1,50$ \\
\hline 313 & 2 & 24 & -95 & 58 & 108 & -98 & 64 & 36,99 & - & $\mathbf{F}^{-}$ & - & 0,00 & $-2,72$ & 0,00 \\
\hline 320 & -22 & 21. & -200 & 117 & 135 & -50 & 91 & 36,72 & - & $\mathrm{V}^{-}$ & $\mathrm{V}^{+}$ & 0,00 & 1,22 & 2,45 \\
\hline 321 & -18 & -26 & -128 & 99 & 129 & -57 & 76 & 39,17 & - & $\mathrm{F}^{+}$ & $\mathrm{V}^{+}$ & 0,00 & $-0,27$ & 0,95 \\
\hline 322 & -15 & -27 & -94 & 77 & 124 & -64 & 67 & 40,12 & - & $\mathrm{V}^{+}$ & $\mathrm{F}^{+}$ & 0,00 & 0,82 & $-0,41$ \\
\hline 323 & -18 & 17 & -87 & 51 & 115 & -78 & 61 & 39,71 & - & $\mathbf{F}^{-}$ & - & 0,00 & $-1,91$ & 0,00 \\
\hline 330 & 3 & & -196 & 94 & 134 & -92 & 96 & 35,50 & - & - & $\mathrm{V}^{+}$ & 0,00 & 0,00 & 3,40 \\
\hline 331 & -8 & 11. & -133 & 72 & 136 & -77 & 73 & 38,90 & - & - & $\mathrm{V}^{+}$ & 0,00 & 0,00 & 2,04 \\
\hline 332 & -14 & 10 & -94 & 50 & 131 & -83 & 64 & 40,94 & - & - & $\mathrm{V}^{+}$ & 0,00 & 0,00 & 0,68 \\
\hline 333 & -16 & 10 & -85 & 34 & 140 & -83 & 61 & 41,62 & - & - & - & 0,00 & 0,00 & 0,00 \\
\hline
\end{tabular}


Apêndice 45. Classificação dos teores foliares segundo o Critério de Faixas de Suficiência, faixas propostas pelo Grupo Paulista de Adubação e Calagem para Citros (1994), diagnose para a correção de N, P e K, resposta líquida da produtividade, diagnose para o nutriente deficiente e a resposta da produtividade, para os dados, dos tratamentos com as doses 0 e $1 \mathrm{de} \mathrm{N}$ em todas as combinações de doses de $\mathrm{P}$ e $\mathrm{K}$, do ensaio de adubação $\mathrm{N}, \mathrm{P}$ e $\mathrm{K}$ conduzido em Araraquara/SP.

\begin{tabular}{|c|c|c|c|c|c|c|c|c|c|c|c|c|c|}
\hline \multirow[b]{2}{*}{ Trat. } & \multicolumn{6}{|c|}{ Classificacão pelo CFS } & \multirow[b]{2}{*}{ Prod. } & \multicolumn{3}{|c|}{ Diagnose } & \multicolumn{3}{|c|}{ Resposta } \\
\hline & $\bar{N}$ & $P$ & $\mathrm{~K}$ & $\mathrm{Ca}$ & $\mathrm{Mg}$ & $\bar{S}$ & & $\mathrm{~N}$ & $P$ & $\mathrm{~K}$ & $\overline{d\left(Y_{N}\right)}$ & $\mathrm{d}\left(\mathrm{Y}_{\mathrm{P}}\right)$ & $\mathrm{d}\left(\mathrm{Y}_{\mathrm{K}}\right)$ \\
\hline & & & & & & & tha ${ }^{-1}$ & & & & & tha $\mathrm{ha}^{-1}$ & \\
\hline 000 & $A D$ & $\mathrm{AD}$ & $A D$ & BA & $\mathrm{AD}$ & $A D$ & 31,33 & $\mathrm{~F}^{-}$ & $\mathrm{V}^{-}$ & $\mathrm{F}^{-}$ & $-3,64$ & 0,00 & $-2,18$ \\
\hline 001 & $\mathrm{AD}$ & $\mathrm{AD}$ & $\mathrm{AD}$ & $\mathrm{BA}$ & $\mathrm{BA}$ & $\mathrm{AD}$ & 33,51 & $\mathrm{~F}^{-}$ & $\mathrm{F}^{-}$ & $\mathrm{F}^{-}$ & $-3,36$ & $-0,44$ & $-1,02$ \\
\hline 002 & $\mathrm{AD}$ & $\mathrm{AD}$ & $\mathrm{AL}$ & $\mathrm{BA}$ & $\mathrm{BA}$ & $\mathrm{AD}$ & 34,53 & $\mathrm{~F}^{-}$ & $\mathrm{F}^{-}$ & $\mathrm{V}^{-}$ & $-2,92$ & $-0,88$ & 0,14 \\
\hline 003 & $\mathrm{AD}$ & $\mathrm{AD}$ & $\mathrm{AL}$ & $\mathrm{BA}$ & $\mathrm{BA}$ & $\mathrm{AD}$ & 34,39 & $\mathrm{~F}^{-}$ & $\mathrm{F}^{-}$ & - & 0,00 & $-1,16$ & 0,00 \\
\hline 010 & $\mathrm{AD}$ & $\mathrm{AD}$ & $\mathrm{AD}$ & $\mathrm{BA}$ & $\mathrm{AD}$ & $\mathrm{AD}$ & 31,33 & $F^{-}$ & $\mathrm{V}^{-}$ & $\mathrm{F}^{-}$ & $-3,35$ & 0,00 & $-2,62$ \\
\hline 011 & $A D$ & $\mathrm{AD}$ & $A D$ & BA & $\mathrm{BA}$ & $\mathrm{AD}$ & 33,95 & $F^{-}$ & $\mathrm{F}^{-}$ & $\mathrm{F}^{-}$ & $-3,06$ & $-0,44$ & $-1,46$ \\
\hline 012 & $A D$ & $A D$ & $\mathrm{AL}$ & BA & $\mathrm{BA}$ & $\mathrm{AD}$ & 35,41 & $\mathrm{~F}^{-}$ & $\mathrm{F}^{-}$ & $\mathrm{F}^{-}$ & $-2,62$ & $-0,73$ & $-0,14$ \\
\hline 013 & $A D$ & $\mathrm{AD}$ & $\mathrm{AL}$ & $\mathrm{BA}$ & $\mathrm{BA}$ & $\mathrm{AD}$ & 35,55 & $\mathrm{~F}^{-}$ & $\mathrm{F}^{-}$ & - & 0,00 & $-1,17$ & 0,00 \\
\hline 020 & $A D$ & $\overline{A D}$ & $A D$ & $\mathrm{BA}$ & $\mathrm{AD}$ & $\mathrm{AD}$ & 31,33 & $\mathrm{~F}$ & $\mathrm{~V}^{-}$ & $\mathrm{F}^{-}$ & $-3,06$ & 0,15 & $-3,06$ \\
\hline 021 & $\mathrm{AD}$ & $\mathrm{AD}$ & $A D$ & BA & $\mathrm{BA}$ & $\mathrm{AD}$ & 34,39 & $\mathrm{~F}^{*}$ & $\mathrm{~F}^{-}$ & $\mathrm{F}^{-}$ & $-2,62$ & $-0,14$ & $-1,75$ \\
\hline 022 & $\mathrm{AD}$ & $\mathrm{AD}$ & $\mathrm{AL}$ & $\mathrm{BA}$ & $\mathrm{BA}$ & $\mathrm{AD}$ & 36,14 & $\mathrm{~F}^{*}$ & $\mathrm{~F}^{-}$ & $F^{*}$ & $-2,33$ & $-0,58$ & $-0,58$ \\
\hline 023 & $A D$ & $A D$ & $\mathrm{AL}$ & BA & BA & $\mathrm{AD}$ & 36,72 & $\mathrm{~F}^{-}$ & $\mathrm{F}^{-}$ & - & 0,00 & $-1,02$ & 0,00 \\
\hline 030 & $A D$ & $\mathrm{AD}$ & $A D$ & BA & $\mathrm{AD}$ & $\mathrm{AD}$ & 31,18 & $\mathrm{~F}^{-}$ & - & $\mathrm{F}^{-}$ & 0,00 & 1,02 & $-3,35$ \\
\hline 031 & $\mathrm{AD}$ & $A D$ & $A D$ & $\mathrm{BA}$ & $\mathrm{AD}$ & $\mathrm{AD}$ & 34,53 & $\mathrm{~F}^{-}$ & - & $\mathrm{F}^{-}$ & 0,00 & 0,58 & $-2,19$ \\
\hline 032 & $\mathrm{AD}$ & $\mathrm{AD}$ & $\overline{\mathrm{AL}}$ & $\mathrm{BA}$ & $\mathrm{BA}$ & $\mathrm{AD}$ & 36,72 & $\mathrm{~F}$ & - & $F^{-}$ & 0,00 & 0,29 & $-1,02$ \\
\hline 033 & $\mathrm{AD}$ & $\mathrm{AD}$ & $\mathrm{AL}$ & BA & BA & $\mathrm{AD}$ & 37,74 & $\mathrm{~F}^{-}$ & - & - & 0,00 & $-0,15$ & 0,00 \\
\hline 100 & $\mathrm{AD}$ & $\overline{A D}$ & $\overline{A D}$ & $\mathrm{BA}$ & $\mathrm{AD}$ & $\mathrm{AD}$ & 34,97 & $\mathrm{~F}^{-}$ & $\mathrm{V}^{*}$ & $F^{-}$ & $-2,33$ & 0,29 & $-1,90$ \\
\hline 101 & $\mathrm{AD}$ & $\mathrm{AD}$ & $A D$ & $\mathrm{BA}$ & $\mathrm{BA}$ & $\mathrm{AD}$ & 36,87 & $\mathrm{~F}^{-}$ & $\mathrm{F}^{-}$ & $\mathrm{F}^{-}$ & $-1,89$ & $-0,14$ & $-0,58$ \\
\hline 102 & $\mathrm{AD}$ & $\mathrm{AD}$ & $A L$ & $\mathrm{BA}$ & $\mathrm{BA}$ & $\mathrm{AD}$ & 37,45 & $\mathrm{~F}^{-}$ & $\mathrm{F}^{-}$ & $\mathrm{V}^{-}$ & $-1,46$ & $-0,58$ & 0,58 \\
\hline 103 & $\mathrm{AD}$ & $\mathrm{AD}$ & $\mathrm{AL}$ & BA & $\mathrm{BA}$ & $\mathrm{AD}$ & 36,87 & $\mathrm{~F}^{-}$ & $\mathrm{F}^{-}$ & - & 0,00 & $-0,87$ & 0,00 \\
\hline 110 & $A D$ & $\mathrm{AD}$ & $A D$ & BA & $\mathrm{AD}$ & $\mathrm{AD}$ & 34,68 & $F^{-}$ & $\mathrm{V}^{-}$ & $\mathrm{F}^{-}$ & $-2,04$ & 0,29 & $-2,33$ \\
\hline 111 & $\mathrm{AD}$ & $\mathrm{AD}$ & $\mathrm{AD}$ & $\mathrm{BA}$ & $\mathrm{BA}$ & $\mathrm{AD}$ & 37,01 & $\mathrm{~F}^{-}$ & $\mathrm{V}^{-}$ & $F$ & $-1,46$ & 0,00 & $-1,02$ \\
\hline 112 & $\mathrm{AD}$ & $\overline{A D}$ & $\mathrm{AL}$ & BA & BA & $\mathrm{AD}$ & 38,03 & $\mathrm{~F}^{-}$ & $\mathrm{F}^{-}$ & $\mathrm{V}^{-}$ & $-1,17$ & $-0,44$ & 0,29 \\
\hline 113 & $\mathrm{AD}$ & $\mathrm{AD}$ & $\mathrm{AL}$ & $\mathrm{BA}$ & $\mathrm{BA}$ & $\mathrm{AD}$ & 37,74 & $\mathrm{~F}^{-}$ & $\mathrm{F}^{-}$ & - & 0,00 & $-0,87$ & 0,00 \\
\hline 120 & $\mathrm{AD}$ & $A D$ & $\mathrm{AD}$ & BA & $\mathrm{AD}$ & $\mathrm{AD}$ & 34,39 & $\mathrm{~F}^{-}$ & $\mathrm{V}^{-}$ & $\mathrm{F}^{-}$ & $-1,60$ & 0,58 & $-2,62$ \\
\hline 121 & $\mathrm{AD}$ & $\mathrm{AD}$ & $A D$ & BA & $\mathrm{BA}$ & $A D$ & 37,01 & $F^{-}$ & $\mathrm{V}^{-}$ & $\mathrm{F}^{-}$ & $-1,17$ & 0,14 & $-1,46$ \\
\hline 122 & $\mathrm{AD}$ & $\mathrm{AD}$ & $\mathrm{AL}$ & BA & BA & $\mathrm{AD}$ & 38,47 & $\mathrm{~F}^{-}$ & $F^{-}$ & $\mathrm{F}^{-}$ & $-0,87$ & $-0,29$ & $-0,14$ \\
\hline 123 & $\mathrm{AD}$ & $\mathrm{AD}$ & $\overline{\mathrm{AL}}$ & BA & $\mathrm{BA}$ & $\mathrm{AD}$ & 38,61 & $\mathrm{~F}^{-}$ & $\mathrm{F}^{-}$ & - & 0,00 & $-0,73$ & 0,00 \\
\hline 130 & $\mathrm{AD}$ & $\mathrm{AD}$ & $A D$ & BA & $\mathrm{AD}$ & $\mathrm{AD}$ & 33,81 & $\mathrm{~F}^{-}$ & - & $F^{-}$ & 0,00 & 1,02 & $-3,06$ \\
\hline 131 & $\mathrm{AD}$ & $\mathrm{AD}$ & $A D$ & BA & $\mathrm{AD}$ & $\mathrm{AD}$ & 36,87 & $\mathrm{~F}^{-}$ & - & $F^{-}$ & 0,00 & 0,58 & $-1,89$ \\
\hline 132 & $\mathrm{AD}$ & $\mathrm{AD}$ & $\mathrm{AL}$ & BA & $\mathrm{BA}$ & $\mathrm{AD}$ & 38,76 & $\mathrm{~F}^{-}$ & - & $F^{-}$ & 0,00 & 0,29 & $-0,58$ \\
\hline 133 & $\mathrm{AD}$ & $\mathrm{AD}$ & $\mathrm{AL}$ & $\mathrm{BA}$ & $\mathrm{BA}$ & $\mathrm{AD}$ & 39,34 & $\mathrm{~F}^{-}$ & - & - & 0,00 & $-0,15$ & 0,00 \\
\hline
\end{tabular}


Apêndice 46. Classificação dos teores foliares segundo o Critério de Faixas de Suficiência, faixas propostas pelo Grupo Paulista de Adubação e Calagem para Citros (1994), diagnose para a correção de $\mathrm{N}, \mathrm{P}$ e K, resposta líquida da produtividade, diagnose para o nutriente deficiente e a resposta da produtividade, para os dados, dos tratamentos com as doses 2 e 3 de $\mathrm{N}$ em todas as combinações de doses de $\mathrm{P}$ e $\mathrm{K}$, do ensaio de adubação $\mathrm{N}, \mathrm{P}$ e $\mathrm{K}$ conduzido em Araraquara/SP.

\begin{tabular}{|c|c|c|c|c|c|c|c|c|c|c|c|c|c|}
\hline \multirow[b]{2}{*}{ Trat. } & \multicolumn{6}{|c|}{ Classificação pelo CFS } & \multirow[b]{2}{*}{ Prod. } & \multicolumn{3}{|c|}{ Diagnose } & \multicolumn{3}{|c|}{ Resposta } \\
\hline & $\bar{N}$ & $\mathrm{P}$ & $\vec{K}$ & $\mathrm{Ca}$ & $\mathrm{Mg}$ & $\bar{S}$ & & $\mathrm{~N}$ & $\mathrm{P}$ & $\mathrm{K}$ & $\bar{d}\left(\mathrm{Y}_{\mathrm{N}}\right)$ & $d\left(Y_{P}\right)$ & $\mathrm{d}\left(\mathrm{Y}_{\mathrm{K}}\right)$ \\
\hline & & & & & & & $\mathrm{tha}^{-1}$ & & & & & $\mathrm{tha}^{-1}$ & \\
\hline 200 & $\mathrm{AD}$ & $\mathrm{AD}$ & $\mathrm{AD}$ & BA & $\mathrm{AD}$ & $\mathrm{AD}$ & 37,30 & $F^{-}$ & $\mathrm{V}^{-}$ & $\mathrm{F}^{-}$ & $-0,88$ & 0,58 & $-1,46$ \\
\hline 201 & $\mathrm{AD}$ & $\mathrm{AD}$ & $\mathrm{AD}$ & BA & $\mathrm{AD}$ & $\mathrm{AD}$ & 38,76 & $F^{-}$ & $\mathrm{V}^{-}$ & $\mathrm{F}^{-}$ & $-0,44$ & 0,29 & $-0,15$ \\
\hline 202 & $A D$ & $\mathrm{AD}$ & $\mathrm{AD}$ & BA & $\mathrm{BA}$ & $\mathrm{AD}$ & 38,91 & $F^{*}$ & $\mathrm{~F}^{-}$ & $\mathrm{V}^{-}$ & $-0,14$ & $-0,29$ & 0,88 \\
\hline 203 & $\mathrm{AD}$ & $\mathrm{AD}$ & $\mathrm{AL}$ & BA & $\mathrm{BA}$ & $\mathrm{AD}$ & 38,03 & $\mathrm{~V}^{*}$ & $\mathrm{~F}^{-}$ & - & 0,00 & $-0,58$ & 0,00 \\
\hline 210 & $\mathrm{AL}$ & $\mathrm{AD}$ & $\mathrm{AD}$ & BA & $\mathrm{AD}$ & $\mathrm{AD}$ & 36,72 & $\mathrm{~F}^{-}$ & $\mathrm{V}^{-}$ & $\mathrm{F}^{-}$ & $-0,44$ & 0,73 & $-1,75$ \\
\hline 211 & $\mathrm{AD}$ & $\mathrm{AD}$ & $A D$ & BA & $\mathrm{BA}$ & $\mathrm{AD}$ & 38,47 & $\mathrm{~F}^{-}$ & $\mathrm{V}^{-}$ & $\mathrm{F}^{-}$ & $-0,29$ & 0,29 & $-0,73$ \\
\hline 212 & $\mathrm{AD}$ & $\mathrm{AD}$ & $\mathrm{AD}$ & BA & BA & $\mathrm{AD}$ & 39,20 & $\mathrm{~V}^{-}$ & $F^{-}$ & $\mathrm{V}^{-}$ & 0,29 & $-0,14$ & 0,59 \\
\hline 213 & $\mathrm{AD}$ & $\mathrm{AD}$ & $\mathrm{AL}$ & $\mathrm{BA}$ & $\mathrm{BA}$ & $A D$ & 38,61 & $\mathrm{~V}^{-}$ & $F^{-}$ & - & 0,00 & $-0,59$ & 0,00 \\
\hline 220 & $\mathrm{AL}$ & $\mathrm{AD}$ & $\mathrm{AD}$ & BA & $\mathrm{AD}$ & $\mathrm{AD}$ & 35,99 & $\mathrm{~F}^{-}$ & $\mathrm{V}^{-}$ & $\mathrm{F}^{-}$ & $-0,15$ & 0,87 & $-2,19$ \\
\hline 221 & $\overline{A D}$ & $\mathrm{AD}$ & $\mathrm{AD}$ & BA & $\mathrm{BA}$ & $\overline{A D}$ & 38,18 & $\mathrm{~V}^{*}$ & $\mathrm{~V}^{-}$ & $\mathrm{F}^{-}$ & 0,15 & 0,29 & $-1,16$ \\
\hline 222 & $\mathrm{AD}$ & $\mathrm{AD}$ & $A D$ & BA & BA & $\mathrm{AD}$ & 39,34 & $\mathrm{~V}^{-}$ & $\mathrm{V}^{-}$ & $\mathrm{V}^{-}$ & 0,58 & 0,00 & 0,14 \\
\hline 223 & $\mathrm{AD}$ & $\mathrm{AD}$ & $\mathrm{AL}$ & BA & BA & $\mathrm{AD}$ & 39,20 & $\mathrm{~V}^{-}$ & $\mathrm{F}^{-}$ & - & 0,00 & $-0,43$ & 0,00 \\
\hline 230 & $\mathrm{AD}$ & $\mathrm{AD}$ & $\mathrm{AD}$ & BA & $\mathrm{AD}$ & $A D$ & 35,12 & $\mathrm{~V}^{\circ}$ & - & $F^{-}$ & 0,00 & 1,02 & $-2,77$ \\
\hline 231 & $\mathrm{AD}$ & $\mathrm{AD}$ & $\mathrm{AD}$ & BA & $\mathrm{AD}$ & $\mathrm{AD}$ & 37,89 & $\mathrm{~V}^{-}$ & - & $\mathrm{F}^{-}$ & 0,00 & 0,58 & $-1,45$ \\
\hline 232 & $\mathrm{AD}$ & $\mathrm{AD}$ & $\mathrm{AD}$ & BA & $\mathrm{BA}$ & $\mathrm{AD}$ & 39,34 & $\mathrm{~V}^{-}$ & - & $\mathrm{F}^{-}$ & 0,00 & 0,29 & $-0,29$ \\
\hline 233 & $\mathrm{AD}$ & $\mathrm{AD}$ & $\mathrm{AL}$ & BA & $\mathrm{BA}$ & $\mathrm{AD}$ & 39,63 & $\mathrm{~V}^{-}$ & - & - & 0,00 & $-0,15$ & 0,00 \\
\hline 300 & $\mathrm{AD}$ & $\mathrm{AD}$ & $A D$ & BA & $\mathrm{AD}$ & $\mathrm{AD}$ & 38,18 & - & $\mathrm{V}^{-}$ & $\mathrm{F}^{-}$ & 0,00 & 1,02 & $-1,02$ \\
\hline 301 & $\mathrm{AD}$ & $\mathrm{AD}$ & $\mathrm{AD}$ & BA & $\mathrm{AD}$ & $\mathrm{AD}$ & 39,20 & - & $\mathrm{V}^{-}$ & $\mathrm{V}^{-}$ & 0,00 & 0,44 & 0,15 \\
\hline 302 & $\mathrm{AD}$ & $\mathrm{AD}$ & $\mathrm{AD}$ & BA & $\mathrm{AD}$ & $\mathrm{AD}$ & 39,05 & - & $\mathrm{V}^{-}$ & $\mathrm{V}^{-}$ & 0,00 & 0,14 & 1,31 \\
\hline 303 & $A D$ & $A D$ & $\mathrm{AL}$ & $\mathrm{BA}$ & $\mathrm{AD}$ & $\mathrm{AD}$ & 37,74 & - & $\mathrm{F}^{-}$ & - & 0,00 & $-0,29$ & 0,00 \\
\hline 310 & $\mathrm{AL}$ & $\mathrm{AD}$ & $\mathrm{AD}$ & BA & $A D$ & $\mathrm{AD}$ & 37,16 & - & $\mathbf{V}^{-}$ & $\mathrm{F}^{-}$ & 0,00 & 1,02 & $-1,60$ \\
\hline 311 & $\mathrm{AD}$ & $\mathrm{AD}$ & $\mathrm{AD}$ & BA & $A D$ & $\mathrm{AD}$ & 38,76 & - & $\mathrm{V}^{-}$ & $\mathrm{F}^{-}$ & 0,00 & 0,73 & $-0,15$ \\
\hline 312 & $\mathrm{AD}$ & $A D$ & $\mathrm{AD}$ & BA & $\mathrm{AD}$ & $A D$ & 38,91 & - & $\mathrm{V}^{-}$ & $\mathrm{V}^{-}$ & 0,00 & 0,15 & 0,88 \\
\hline 313 & $\mathrm{AD}$ & $\mathrm{AD}$ & $\mathrm{AL}$ & BA & $A D$ & $\mathrm{AD}$ & 38,03 & - & $\mathrm{F}^{-}$ & - & 0,00 & $-0,29$ & 0,00 \\
\hline 320 & $\mathrm{AL}$ & $A D$ & $\mathrm{AD}$ & BA & $A D$ & $\mathrm{AD}$ & 36,14 & - & $\mathrm{V}^{-}$ & $\mathrm{F}^{-}$ & 0,00 & 1,02 & $-1,89$ \\
\hline 321 & $\mathrm{AD}$ & $\mathrm{AD}$ & $\mathrm{AD}$ & BA & $\mathrm{AD}$ & $\mathrm{AD}$ & 38,03 & - & $\mathrm{V}^{-}$ & $\mathrm{F}^{-}$ & 0,00 & 0,58 & $-0,73$ \\
\hline 322 & $\mathrm{AD}$ & $\mathrm{AD}$ & $\mathrm{AD}$ & $\mathrm{BA}$ & $A D$ & $\mathrm{AD}$ & 38,76 & - & $\mathrm{V}^{-}$ & $\mathrm{V}^{-}$ & 0,00 & 0,29 & 0,44 \\
\hline 323 & $\mathrm{AD}$ & $\mathrm{AD}$ & $\mathrm{AL}$ & BA & $A D$ & $\mathrm{AD}$ & 38,32 & - & $\mathrm{F}^{-}$ & - & 0,00 & $-0,15$ & 0,00 \\
\hline 330 & $\mathrm{AD}$ & $\mathrm{AD}$ & $\mathrm{AD}$ & BA & $\mathrm{AD}$ & $\mathrm{AD}$ & 35,12 & - & - & $\mathrm{F}^{-}$ & 0,00 & 1,02 & $-2,33$ \\
\hline 331 & $\mathrm{AD}$ & $\mathrm{AD}$ & $\mathrm{AD}$ & $\mathrm{BA}$ & $\mathrm{AD}$ & $\overline{A D}$ & 37,45 & - & - & $\mathrm{F}^{-}$ & 0,00 & 0,58 & $-1,02$ \\
\hline 332 & $\mathrm{AD}$ & $\mathrm{AD}$ & $\mathrm{AD}$ & BA & $A D$ & $\mathrm{AD}$ & 38,47 & - & - & $\mathrm{V}^{*}$ & 0,00 & 0,29 & 0,00 \\
\hline 333 & $\mathrm{AD}$ & $\mathrm{AD}$ & $\mathrm{Al}$ & BA & $\mathrm{AD}$ & $\mathrm{AD}$ & 38,47 & - & - & - & 0,00 & $-0,15$ & 0,00 \\
\hline
\end{tabular}


Apêndice 47. Classificação dos teores foliares segundo o Critério de Faixas de Suficiência, faixas propostas pelo Grupo Paulista de Adubação e Calagem para Citros (1994), diagnose para a correção de N, P e K, resposta líquida da produtividade, diagnose para o nutriente deficiente e a resposta da produtividade, para os dados, dos tratamentos com as doses 0 e 1 de $\mathrm{N}$ em todas as combinações de doses de $\mathrm{P}$ e $\mathrm{K}$, do ensaio de adubação $\mathrm{N}, \mathrm{P}$ e $\mathrm{K}$ conduzido em Botucatu/SP.

\begin{tabular}{|c|c|c|c|c|c|c|c|c|c|c|c|c|}
\hline \multirow[b]{2}{*}{ Trat. } & \multicolumn{5}{|c|}{ Classificacão pelo CFS } & \multirow[b]{2}{*}{ Prod. } & \multicolumn{3}{|c|}{ Diagnose } & \multicolumn{3}{|c|}{ Resposta } \\
\hline & $\mathrm{N}$ & $\mathrm{P}$ & $\mathrm{K}$ & $\mathrm{Ca}$ & $\mathrm{Mg}$ & & $\mathrm{N}$ & $\mathrm{P}$ & $\mathrm{K}$ & $\mathrm{d}\left(\mathrm{Y}_{\mathrm{N}}\right)$ & $d\left(Y_{p}\right)$ & $\mathrm{d}\left(\mathrm{Y}_{\mathrm{K}}\right)$ \\
\hline & & & & & & $\mathrm{tha}^{-1}$ & & & & & that & \\
\hline 000 & $\mathrm{BA}$ & $\mathrm{AD}$ & $\mathrm{BA}$ & $\mathrm{AD}$ & $\mathrm{AL} \quad \mathrm{AD}$ & 36,04 & $\mathrm{~V}^{+}$ & $\mathrm{F}^{-}$ & $\mathrm{V}^{+}$ & 4,08 & $-0,76$ & 4,93 \\
\hline 001 & $\mathrm{BA}$ & $\mathrm{AD}$ & $\mathrm{BA}$ & $\mathrm{AD}$ & $\mathrm{AL} \quad \mathrm{AD}$ & 40,97 & $\mathrm{~V}^{+}$ & $\mathrm{F}^{-}$ & $\mathrm{V}^{+}$ & 4,42 & $-0,76$ & 2,29 \\
\hline 002 & $\mathrm{BA}$ & $\mathrm{AD}$ & $\mathrm{AD}$ & $\mathrm{BA}$ & $\mathrm{AD} \mathrm{AD}$ & 43,26 & $\mathrm{~V}^{+}$ & $\mathrm{F}^{-}$ & $\mathrm{V}^{-}$ & 4,77 & $-0,69$ & 0,42 \\
\hline 003 & BA & $\mathrm{AD}$ & $\mathrm{AL}$ & $\mathrm{BA}$ & $\mathrm{AD} \mathrm{AD}$ & 42,84 & $\mathrm{~V}^{+}$ & $\mathrm{F}^{-}$ & - & 0,00 & $-0,68$ & 0,51 \\
\hline 010 & $\mathrm{BA}$ & $\mathrm{AD}$ & $\mathrm{BA}$ & $\mathrm{AD}$ & $\mathrm{AL} \quad \mathrm{AD}$ & 36,80 & $\mathrm{~V}^{+}$ & $\mathrm{F}^{-}$ & $\mathrm{V}^{+}$ & 4,51 & $-1,71$ & 4,93 \\
\hline 011 & BA & $\mathrm{AD}$ & $\mathrm{BA}$ & $\mathrm{AD}$ & $\mathrm{AL} \quad \mathrm{AD}$ & 41,73 & $\mathrm{~V}^{+}$ & $\mathrm{F}^{-}$ & $\mathrm{V}^{+}$ & 4,85 & $-1,71$ & 2,22 \\
\hline 012 & $\mathrm{BA}$ & $\mathrm{AD}$ & $\mathrm{AD}$ & $\mathrm{BA}$ & $\mathrm{AL} \quad \mathrm{AD}$ & 43,95 & $\mathrm{~V}^{+}$ & $\mathrm{F}^{-}$ & $\mathrm{V}^{-}$ & 5,27 & $-1,70$ & 0,43 \\
\hline 013 & $\mathrm{BA}$ & $\mathrm{AD}$ & $\mathrm{AL}$ & $\mathrm{BA}$ & $A D A D$ & 43,52 & $\mathrm{~V}^{+}$ & $\mathrm{F}^{-}$ & - & 0,00 & $-1,61$ & 0,51 \\
\hline 020 & $\mathrm{BA}$ & $\mathrm{AD}$ & $\mathrm{BA}$ & $\mathrm{AL}$ & $\mathrm{AL} \quad \mathrm{AD}$ & 38,51 & $\mathrm{~V}^{+}$ & $\mathrm{F}^{-}$ & $\mathrm{V}^{+}$ & 5,01 & $-2,72$ & 4,93 \\
\hline 021 & $\mathrm{BA}$ & $\mathrm{AD}$ & $\mathrm{BA}$ & $\mathrm{AD}$ & $\mathrm{AL} \quad \mathrm{AD}$ & 43,44 & $\mathrm{~V}^{+}$ & $\mathrm{F}^{-}$ & $\mathrm{V}^{+}$ & 5,35 & $-2,72$ & 2,21 \\
\hline 022 & $\mathrm{BA}$ & $\mathrm{AD}$ & $\mathrm{AD}$ & $\mathrm{AD}$ & $\mathrm{AL} \mathrm{AD}$ & 45,65 & $\mathrm{~V}^{+}$ & $\mathrm{F}^{-}$ & $\mathrm{V}^{-}$ & 5,69 & $-2,72$ & 0,52 \\
\hline 023 & BA & $\mathrm{AD}$ & $\mathrm{AD}$ & $\mathrm{BA}$ & $\mathrm{AD} A D$ & 45,13 & $\mathrm{~V}^{+}$ & $\mathrm{F}^{-}$ & - & 0,00 & $-2,72$ & 0,51 \\
\hline 030 & BA & $\mathrm{AD}$ & $\mathrm{BA}$ & $\mathrm{AL}$ & $\mathrm{AL} \quad \mathrm{AD}$ & 41,23 & $\mathrm{~V}^{+}$ & - & $\mathrm{V}^{+}$ & 0,00 & $-4,09$ & 4,93 \\
\hline 031 & $\mathrm{BA}$ & $\mathrm{AD}$ & BA & $\mathrm{AD}$ & $\mathrm{AL} \quad \mathrm{AD}$ & 46,16 & $\mathrm{~V}^{+}$ & - & $\mathrm{V}^{+}$ & 0,00 & $-4,07$ & 2,21 \\
\hline 032 & $\mathrm{BA}$ & $\mathrm{AD}$ & $\mathrm{AD}$ & $\mathrm{AD}$ & $\mathrm{AL} \quad \mathrm{AD}$ & 48,37 & $\mathrm{~V}^{+}$ & - & $\mathrm{V}^{-}$ & 0,00 & $-4,09$ & 0,52 \\
\hline 033 & $\mathrm{BA}$ & $\mathrm{AD}$ & $\mathrm{AD}$ & $\mathrm{BA}$ & $\mathrm{AL} \quad \mathrm{AD}$ & 47,85 & $\mathrm{~V}^{+}$ & - & - & 0,00 & $-4,00$ & 0,51 \\
\hline 100 & $\mathrm{BA}$ & $\mathrm{AD}$ & $\mathrm{BA}$ & $\mathrm{AD}$ & $\mathrm{AL} \quad \mathrm{AD}$ & 40,12 & $\mathrm{~V}^{+}$ & $\mathrm{F}^{-}$ & $\mathrm{V}^{+}$ & 1,28 & $-1,19$ & 5,27 \\
\hline 101 & $\mathrm{BA}$ & $\mathrm{AD}$ & $\mathrm{BA}$ & $\mathrm{AD}$ & $\mathrm{AL} \quad \mathrm{AD}$ & 45,39 & $\mathrm{~V}^{+}$ & $\mathrm{F}^{-}$ & $\mathrm{V}^{+}$ & 1,70 & $-1,19$ & 2,64 \\
\hline 102 & $\mathrm{BA}$ & $A D$ & $\mathrm{BA}$ & $\mathrm{BA}$ & $\mathrm{AL} \quad \mathrm{AD}$ & 48,03 & $\mathrm{~V}^{+}$ & $\mathrm{F}^{-}$ & $\mathrm{F}^{+}$ & 2,03 & $-1,19$ & $-0,09$ \\
\hline 103 & $\mathrm{BA}$ & $\mathrm{AD}$ & $\mathrm{AD}$ & $\mathrm{BA}$ & $\mathrm{AD} \mathrm{AD}$ & 47,94 & $\mathrm{~V}^{+}$ & $\mathrm{F}^{-}$ & - & 0,00 & $-1,10$ & 0,51 \\
\hline 110 & $\mathrm{BA}$ & $\mathrm{AD}$ & $\mathrm{BA}$ & $\mathrm{AL}$ & $\mathrm{AL} \quad \mathrm{AD}$ & 41,31 & $\mathrm{~V}^{+}$ & $\mathrm{F}^{-}$ & $\mathrm{V}^{+}$ & 1,78 & $-2,21$ & 5,27 \\
\hline 111 & $\mathrm{BA}$ & $A D$ & $\mathrm{BA}$ & $\mathrm{AD}$ & $\mathrm{AL} \quad \mathrm{AD}$ & 46,58 & $\mathrm{~V}^{+}$ & $\mathrm{F}^{-}$ & $\mathrm{V}^{+}$ & 2,13 & $-2,21$ & 2,64 \\
\hline 112 & BA & $\mathrm{AD}$ & $\mathrm{BA}$ & $\mathrm{AD}$ & $\mathrm{AL} \quad \mathrm{AD}$ & 49,22 & $\mathrm{~V}^{+}$ & $\mathrm{F}^{-}$ & $\mathrm{F}^{+}$ & 2,37 & $-2,12$ & $-0,18$ \\
\hline 113 & $\mathrm{BA}$ & $\mathrm{AD}$ & $\mathrm{AD}$ & $\mathrm{BA}$ & $\mathrm{AL} \quad \mathrm{AD}$ & 49,04 & $\mathrm{~V}^{+}$ & $\mathrm{F}^{-}$ & - & 0,00 & $-2,13$ & 0,51 \\
\hline 120 & BA & $\mathrm{AD}$ & BA & $\mathrm{AL}$ & $\mathrm{AL} \quad \mathrm{AD}$ & 43,52 & $\mathrm{~V}^{+}$ & $\mathbf{F}^{-}$ & $\mathrm{V}^{+}$ & 2,21 & $-3,14$ & 5,27 \\
\hline 121 & $\mathrm{BA}$ & $\mathrm{AD}$ & $\mathrm{BA}$ & $\mathrm{AD}$ & $\mathrm{AL} \quad \mathrm{AD}$ & 48,79 & $\mathrm{~V}^{+}$ & $F^{-}$ & $\mathrm{V}^{+}$ & 2,55 & $-3,15$ & 2,55 \\
\hline 122 & BA & $A D$ & BA & $\mathrm{AD}$ & $\mathrm{AL} \quad \mathrm{AD}$ & 51,34 & $\mathrm{~V}^{+}$ & $F^{-}$ & $\mathrm{F}^{+}$ & 2,89 & $-3,15$ & $-0,17$ \\
\hline 123 & $\mathrm{BA}$ & $\mathrm{AD}$ & $\mathrm{AD}$ & $\mathrm{BA}$ & $\mathrm{AL} \quad \mathrm{AD}$ & 51,17 & $\mathrm{~V}^{+}$ & $\mathrm{F}^{-}$ & - & 0,00 & $-3,14$ & 0,51 \\
\hline 130 & $\mathrm{BA}$ & $\mathrm{AD}$ & $\mathrm{BA}$ & $\mathrm{AL}$ & $\mathrm{AL} \quad \mathrm{AD}$ & 46,66 & $\mathrm{~V}^{+}$ & - & $\mathrm{V}^{+}$ & 0,00 & $-4,09$ & 5,28 \\
\hline 131 & $\mathrm{BA}$ & $\mathrm{AD}$ & $\mathrm{BA}$ & $\overline{A D}$ & $\overline{A L} \quad \overline{A D}$ & 51,94 & $\mathrm{~V}^{+}$ & - & $\mathrm{V}^{+}$ & 0,00 & $-4,07$ & 2,55 \\
\hline 132 & $\mathrm{BA}$ & $\mathrm{AD}$ & $\mathrm{BA}$ & $\mathrm{AD}$ & $\mathrm{AL} \quad \mathrm{AD}$ & 54,49 & $\mathrm{~V}^{+}$ & - & $\mathrm{F}^{+}$ & 0,00 & $-4,09$ & $-0,18$ \\
\hline 133 & $\mathrm{BA}$ & $\mathrm{AD}$ & $\mathrm{AD}$ & BA & $\mathrm{AL} \quad \mathrm{AD}$ & 54,31 & $\mathrm{~V}^{+}$ & - & - & 0,00 & $-4,00$ & 0,51 \\
\hline
\end{tabular}


Apêndice 48. Classificação dos teores foliares segundo o Critério de Faixas de Suficiência, faixas propostas pelo Grupo Paulista de Adubação e Calagem para Citros (1994), diagnose para a correção de $\mathrm{N}, \mathrm{P}$ e K, resposta líquida da produtividade, diagnose para o nutriente deficiente e a resposta da produtividade, para os dados, dos tratamentos com as doses 2 e 3 de $\mathrm{N}$ em todas as combinaçães de doses de $P$ e $K$, do ensaio de adubação $N, P$ e $K$ conduzido em Botucatu/SP.

\begin{tabular}{|c|c|c|c|c|c|c|c|c|c|c|c|c|}
\hline \multirow[b]{2}{*}{ Trat. } & \multicolumn{5}{|c|}{ Classificacão pelo CFS } & \multirow[b]{2}{*}{ Prod. } & \multicolumn{3}{|c|}{ Diagnose } & \multicolumn{3}{|c|}{ Resposta } \\
\hline & $\mathrm{N}$ & $P$ & $\mathrm{~K}$ & $\mathrm{Ca}$ & $\mathrm{Mg}$ & & $\mathrm{N}$ & $P$ & $\mathrm{~K}$ & $d\left(Y_{N}\right)$ & $d\left(Y_{P}\right)$ & $\mathrm{d}\left(\mathrm{Y}_{\mathrm{K}}\right)$ \\
\hline & & & & & & $\mathrm{tha}^{-1}$ & & & & & tha ${ }^{-1}$ & \\
\hline 200 & $\mathrm{AD}$ & $\mathrm{AD}$ & $\mathrm{BA}$ & $\mathrm{AD}$ & $\mathrm{AL} \quad \mathrm{AD}$ & 41,40 & $\mathrm{~V}^{-}$ & $\mathrm{F}^{-}$ & $\mathrm{V}^{+}$ & 1,45 & $-1,69$ & 5,69 \\
\hline 201 & $\mathrm{BA}$ & $\mathrm{AD}$ & $\mathrm{BA}$ & $\mathrm{AD}$ & AL AD & 47,09 & $\mathrm{~F}^{+}$ & $F^{-}$ & $\mathrm{V}^{+}$ & $-1,19$ & $-1,62$ & 2,97 \\
\hline 202 & $\mathrm{BA}$ & $\mathrm{AD}$ & $\mathrm{BA}$ & $\mathrm{BA}$ & $\mathrm{AL} \mathrm{AD}$ & 50,06 & $\mathrm{~F}^{+}$ & $F^{-}$ & $\mathrm{V}^{+}$ & $-0,84$ & $-1,53$ & 0,26 \\
\hline 203 & $\mathrm{AD}$ & $\mathrm{AD}$ & $\mathrm{AD}$ & $\mathrm{BA}$ & $\mathrm{AL} \quad \mathrm{AD}$ & 50,32 & $\mathrm{~V}^{-}$ & $\mathrm{F}^{-}$ & - & 0,00 & $-1,53$ & 0,51 \\
\hline 210 & $\mathrm{BA}$ & $\mathrm{AD}$ & $\mathrm{BA}$ & $\mathrm{AL}$ & $\mathrm{AL} A \mathrm{AD}$ & 43,09 & $\mathrm{~F}^{+}$ & $\mathrm{F}$ & $\mathrm{V}^{+}$ & $-1,10$ & $-2,64$ & 5,62 \\
\hline 211 & $\mathrm{BA}$ & $\mathrm{AD}$ & BA & $\mathrm{AD}$ & $\mathrm{AL} \quad \mathrm{AD}$ & 48,71 & $\mathrm{~F}^{+}$ & $F^{-}$ & $\mathrm{V}^{+}$ & $-0,68$ & $-2,63$ & 2,88 \\
\hline 212 & $\mathrm{BA}$ & $\mathrm{AD}$ & BA & $\overline{A D}$ & $\mathrm{AL} \quad \overline{\mathrm{AD}}$ & 51,59 & $\mathrm{~F}^{+}$ & $\mathrm{F}^{-}$ & $\mathrm{V}^{+}$ & $-0,33$ & $-2,64$ & 0,26 \\
\hline 213 & $\mathrm{BA}$ & $\mathrm{AD}$ & $\mathrm{AD}$ & $\mathrm{BA}$ & $\mathrm{AL} \mathrm{AD}$ & 51,85 & $\mathrm{~F}^{+}$ & $\mathrm{F}^{-}$ & - & 0,00 & $-2,64$ & 0,51 \\
\hline 220 & $\mathrm{BA}$ & $\mathrm{AD}$ & $\mathrm{BA}$ & $\mathrm{AL}$ & $\mathrm{AL} \mathrm{AD}$ & 45,73 & $\mathrm{~F}^{+}$ & $\mathrm{F}^{-}$ & $\mathrm{V}^{+}$ & $-0,60$ & $-3,57$ & 5,61 \\
\hline 221 & BA & $\mathrm{AD}$ & $\mathrm{BA}$ & $\mathrm{AD}$ & $\mathrm{AL} \quad \mathrm{AD}$ & 51,34 & $\mathrm{~F}^{+}$ & $F^{-}$ & $\mathrm{V}^{+}$ & $-0,25$ & $-3,57$ & 2,89 \\
\hline 222 & $\mathrm{BA}$ & $\mathrm{AD}$ & $\mathrm{BA}$ & $\mathrm{AD}$ & $\mathrm{AL} \quad \mathrm{AD}$ & 54,23 & $\mathrm{~V}^{+}$ & $\mathrm{F}^{-}$ & $\mathrm{V}^{+}$ & 0,08 & $-3,57$ & 0,26 \\
\hline 223 & BA & $\mathrm{AD}$ & $\mathrm{AD}$ & $\mathrm{BA}$ & $\mathrm{AL} \quad \mathrm{AD}$ & 54,49 & $\mathrm{~V}^{+}$ & $\mathrm{F}^{-}$ & - & 0,00 & $-3,48$ & 0,51 \\
\hline 230 & BA & $\mathrm{AD}$ & $\mathrm{BA}$ & $\mathrm{AL}$ & $\mathrm{AL} \mathrm{AD}$ & 49,30 & $\mathrm{~F}^{+}$ & - & $\mathrm{V}^{+}$ & 0,00 & $-4,09$ & 5,61 \\
\hline 231 & $\mathrm{BA}$ & $\mathrm{AD}$ & $\mathrm{BA}$ & $\mathrm{AD}$ & $\mathrm{AL} \mathrm{AD}$ & 54,91 & $\mathrm{~V}^{+}$ & - & $\mathrm{V}^{+}$ & 0,00 & $-4,07$ & 2,89 \\
\hline 232 & $\mathrm{BA}$ & $\mathrm{AD}$ & $\mathrm{BA}$ & $\mathrm{AD}$ & $\mathrm{AL} \quad \mathrm{AD}$ & 57,80 & $\mathrm{~V}^{+}$ & - & $\mathrm{V}^{+}$ & 0,00 & $-4,09$ & 0,17 \\
\hline 233 & $\mathrm{AD}$ & $\mathrm{AD}$ & $\mathrm{AD}$ & $\mathrm{BA}$ & $A L A D$ & 57,97 & $\mathrm{~F}^{-}$ & - & - & 0,00 & $-4,00$ & 0,51 \\
\hline 300 & $\mathrm{AD}$ & $\mathrm{AD}$ & $\mathrm{BA}$ & $\mathrm{AD}$ & AL BA & 39,95 & - & $\mathrm{F}^{-}$ & $\mathrm{V}^{+}$ & 0,00 & $-2,04$ & 5,95 \\
\hline 301 & $\mathrm{AD}$ & $\mathrm{AD}$ & $\mathrm{BA}$ & $\mathrm{AD}$ & $\mathrm{AL} \mathrm{BA}$ & 45,90 & - & $\mathrm{F}^{-}$ & $\mathrm{V}^{+}$ & 0,00 & $-2,13$ & 3,32 \\
\hline 302 & $\mathrm{AD}$ & $\mathrm{AD}$ & $\mathrm{BA}$ & $\mathrm{BA}$ & AL BA & 49,22 & - & $\mathrm{F}^{-}$ & $\mathrm{V}^{+}$ & 0,00 & $-2,04$ & 0,59 \\
\hline 303 & $\mathrm{AD}$ & $\mathrm{AD}$ & $\mathrm{AD}$ & $\mathrm{BA}$ & $\mathrm{AL} \quad \mathrm{AD}$ & 49,81 & - & $\mathrm{F}^{-}$ & - & 0,00 & $-2,04$ & 0,51 \\
\hline 310 & $\mathrm{AD}$ & $\mathrm{AD}$ & $\mathrm{BA}$ & $\mathrm{AL}$ & $\mathrm{AL} \quad \mathrm{BA}$ & 41,99 & - & $\mathrm{F}^{-}$ & $\mathrm{V}^{+}$ & 0,00 & $-3,14$ & 6,04 \\
\hline 311 & $\mathrm{BA}$ & $\mathrm{AD}$ & $\mathrm{BA}$ & $\mathrm{AD}$ & $\mathrm{AL} \mathrm{BA}$ & 48,03 & - & $\mathrm{F}^{-}$ & $\mathrm{V}^{+}$ & 0,00 & $-3,06$ & 3,23 \\
\hline 312 & BA & $\mathrm{AD}$ & $\mathrm{BA}$ & $\mathrm{AD}$ & $\mathrm{AL} \quad \mathrm{BA}$ & 51,26 & - & $\mathrm{F}^{-}$ & $\mathrm{V}^{+}$ & 0,00 & $-3,05$ & 0,59 \\
\hline 313 & $\mathrm{AD}$ & $\mathrm{AD}$ & $\mathrm{AD}$ & $\mathrm{BA}$ & $\mathrm{AL} \quad \mathrm{AD}$ & 51,85 & - & $\mathrm{F}^{-}$ & - & 0,00 & $-3,06$ & 0,51 \\
\hline 320 & BA & $\mathrm{AD}$ & $\mathrm{BA}$ & $\mathrm{AL}$ & AL BA & 45,13 & - & $\mathrm{F}^{-}$ & $\mathrm{V}^{+}$ & 0,00 & $-4,09$ & 5,96 \\
\hline 321 & BA & $\mathrm{AD}$ & $\mathrm{BA}$ & $A D$ & $\mathrm{AL} \quad \mathrm{BA}$ & 51,09 & - & $\mathrm{F}^{*}$ & $\mathrm{~V}^{+}$ & 0,00 & $-4,07$ & 3,22 \\
\hline 322 & BA & $\mathrm{AD}$ & $\mathrm{BA}$ & $\mathrm{AD}$ & AL BA & 54,31 & - & $\mathrm{F}^{-}$ & $\mathrm{V}^{+}$ & 0,00 & $-4,09$ & 0,60 \\
\hline 323 & $\mathrm{AD}$ & $\mathrm{AD}$ & $\mathrm{AD}$ & $\mathrm{BA}$ & $\mathrm{AL} A \mathrm{AD}$ & 54,91 & - & $\mathrm{F}^{-}$ & - & 0,00 & $-4,00$ & 0,51 \\
\hline 330 & $\mathrm{AD}$ & $\mathrm{AD}$ & $\mathrm{BA}$ & $\mathrm{AL}$ & $\mathrm{AL} \mathrm{BA}$ & 49,22 & - & - & $\mathrm{V}^{+}$ & 0,00 & $-4,09$ & 5,94 \\
\hline 331 & BA & $\mathrm{AD}$ & $\mathrm{BA}$ & $\mathrm{AD}$ & $\mathrm{AL} \mathrm{BA}$ & 55,16 & - & - & $\mathrm{V}^{+}$ & 0,00 & $-4,07$ & 3,24 \\
\hline 332 & $\mathrm{AD}$ & $A D$ & $\mathrm{BA}$ & $\mathrm{AD}$ & $\mathrm{AL} \quad \mathrm{BA}$ & 58,40 & - & - & $\mathrm{V}^{+}$ & 0,00 & $-4,09$ & 0,51 \\
\hline 333 & $\mathrm{AD}$ & $\mathrm{AD}$ & $\mathrm{BA}$ & $\mathrm{AD}$ & $\mathrm{AL} \mathrm{BA}$ & 58,91 & - & - & - & 0,00 & $-4,00$ & 0,51 \\
\hline
\end{tabular}


Apêndice 49. Classificação dos teores foliares segundo o Critério de Faixas de Suficiência, faixas propostas pelo Grupo Paulista de Adubação e Calagem para Citros (1994), diagnose para a correção de $\mathrm{N}, \mathrm{P}$ e $\mathrm{K}$. resposta líquida da produtividade, diagnose para o nutriente deficiente e a resposta da produtividade, para os dados, dos tratamentos com as doses 0 e 1 de $\mathrm{N}$ em todas as combinações de doses de $\mathrm{P}$ e $\mathrm{K}$, do ensaio de adubação $\mathrm{N}, \mathrm{P}$ e $\mathrm{K}$ conduzido em Matão/SP.

\begin{tabular}{|c|c|c|c|c|c|c|c|c|c|c|c|}
\hline \multirow[b]{2}{*}{ Trat. } & \multicolumn{4}{|c|}{ Classificacão pelo CFS } & \multirow[b]{2}{*}{ Prod. } & \multicolumn{3}{|c|}{ Diagnose } & \multicolumn{3}{|c|}{ Resposta } \\
\hline & $\mathrm{N}$ & $P$ & $\mathrm{~K} \mathrm{Ca}$ & $\mathrm{Mg}$ & & $\mathrm{N}$ & $\mathrm{P}$ & $\mathrm{K}$ & $d\left(Y_{N}\right)$ & $\mathrm{d}\left(\mathrm{Y}_{\mathrm{P}}\right)$ & $\mathrm{d}\left(\mathrm{Y}_{\mathrm{K}}\right)$ \\
\hline & & & & & $\mathrm{tha}^{-1}$ & & & & & $-\mathrm{tha}^{-1}$ & \\
\hline 000 & $\mathrm{AD}$ & $\mathrm{AD}$ & $\mathrm{AD} \mathrm{AD}$ & $\mathrm{AL} A D$ & 34,77 & $\mathrm{~F}^{-}$ & $\mathrm{F}^{-}$ & $\mathrm{V}^{-}$ & $-2,21$ & $-4,75$ & 1,54 \\
\hline 001 & $\mathrm{AD}$ & $\mathrm{AD}$ & $\mathrm{AL} \quad \mathrm{AD}$ & $\mathrm{AL} \mathrm{AD}$ & 33,23 & $\mathrm{~F}^{-}$ & $\mathrm{F}^{-}$ & $\mathrm{F}^{-}$ & $-2,04$ & $-4,34$ & $-0,35$ \\
\hline 002 & $\mathrm{AD}$ & $\mathrm{AD}$ & $\mathrm{AL} \quad \mathrm{AD}$ & $\mathrm{AD} \mathrm{AD}$ & 33,58 & $\mathrm{~F}^{-}$ & $\mathrm{F}^{-}$ & $\mathrm{F}^{-}$ & $-1,95$ & $-3,90$ & $-2,21$ \\
\hline 003 & $\mathrm{AD}$ & $\mathrm{AD}$ & $\mathrm{AL} \quad \mathrm{BA}$ & $\mathrm{AD} \mathrm{AD}$ & 35,79 & $\mathrm{~F}^{-}$ & $\mathrm{F}^{-}$ & - & 0,00 & $-3,48$ & $-0,59$ \\
\hline 010 & $\mathrm{AD}$ & $\mathrm{AD}$ & $\mathrm{AL} \quad \mathrm{AD}$ & $\mathrm{AL} A D$ & 39,52 & $\mathrm{~F}^{-}$ & $\mathrm{F}^{-}$ & $\mathrm{V}^{*}$ & $-2,30$ & $-2,47$ & 1,95 \\
\hline 011 & AD & $\mathrm{AD}$ & $\mathrm{AL} \quad \mathrm{AD}$ & $A L A D$ & 37,57 & $\mathrm{~F}^{-}$ & $\mathrm{F}^{-}$ & $\mathrm{V}^{-}$ & $-2,13$ & $-2,04$ & 0,09 \\
\hline 012 & $A D$ & $\mathrm{AD}$ & $\mathrm{AL} \quad \mathrm{AD}$ & $\mathrm{AD} \quad \mathrm{AD}$ & 37,48 & $\mathrm{~F}^{-}$ & $\mathrm{F}^{-}$ & $\mathrm{F}^{-}$ & $-2,04$ & $-1,54$ & $-1,79$ \\
\hline 013 & $\mathrm{AD}$ & $\mathrm{AD}$ & $\mathrm{AL} \quad \mathrm{BA}$ & $A D A D$ & 39,27 & $\mathrm{~F}^{-}$ & $\mathrm{F}^{-}$ & - & 0,00 & $-1,11$ & $-0,59$ \\
\hline 020 & $\mathrm{AD}$ & $\mathrm{AD}$ & $\mathrm{AL} A \mathrm{D}$ & $\mathrm{AL} A \mathrm{D}$ & 41,99 & $\mathrm{~F}^{-}$ & $\mathrm{F}^{-}$ & $\mathrm{V}^{-}$ & $-2,38$ & $-0,09$ & 2,38 \\
\hline 021 & $\mathrm{AD}$ & $\mathrm{AD}$ & $\mathrm{AL} \quad \mathrm{AD}$ & $\mathrm{AL} \quad \mathrm{AD}$ & 39,61 & $\mathrm{~F}^{*}$ & $\mathrm{~V}^{-}$ & $\mathrm{V}^{*}$ & $-2,21$ & 0,34 & 0,59 \\
\hline 022 & $\mathrm{AD}$ & $\mathrm{AD}$ & $\mathrm{AL} \quad \mathrm{AD}$ & $\mathrm{AL} A D$ & 39,02 & $\mathrm{~F}^{-}$ & $\mathrm{V}^{-}$ & $\mathrm{F}^{-}$ & $-2,12$ & 0,77 & $-1,36$ \\
\hline 023 & $\mathrm{AD}$ & $A D$ & $\mathrm{AL} \quad \mathrm{AD}$ & $\mathrm{AD} A D$ & 40,38 & $\mathrm{~F}^{-}$ & $\mathrm{V}^{-}$ & - & 0,00 & 1,19 & $-0,59$ \\
\hline 030 & $A D$ & $\mathrm{AD}$ & $\mathrm{AD} \quad \mathrm{AD}$ & $\mathrm{AL} \quad \mathrm{AD}$ & 42,08 & $\mathrm{~F}^{-}$ & - & $\mathrm{V}^{-}$ & 0,00 & $-0,43$ & 2,81 \\
\hline 031 & $\mathrm{AD}$ & $\overline{A D}$ & $\mathrm{AL} \mathrm{AD}$ & $\overline{A L} \quad \overline{A D}$ & 39,27 & $\mathrm{~F}^{-}$ & - & $\mathrm{V}^{-}$ & 0,00 & 0,08 & 1,02 \\
\hline 032 & $\mathrm{AD}$ & $\mathrm{AD}$ & $\mathrm{AL} \quad \mathrm{AD}$ & $\mathrm{AL} \quad \mathrm{AD}$ & 38,25 & $\mathrm{~F}^{-}$ & - & $\mathrm{F}^{-}$ & 0,00 & 0,51 & $-0,94$ \\
\hline 033 & $\mathrm{AD}$ & $\mathrm{AD}$ & $\mathrm{AL} \quad \mathrm{AD}$ & $\mathrm{AD} A \mathrm{AD}$ & 39,19 & $\mathrm{~F}^{-}$ & - & - & 0,00 & 0,94 & $-0,59$ \\
\hline 100 & $\mathrm{AD}$ & $A D$ & $\mathrm{AD} A D$ & $\mathrm{AL} \quad \mathrm{AD}$ & 36,98 & $\mathrm{~F}^{-}$ & $\mathrm{F}^{-}$ & $\mathrm{V}^{-}$ & $-1,01$ & $-4,84$ & 1,71 \\
\hline 101 & $\mathrm{AD}$ & $\mathrm{AD}$ & $\mathrm{AD} A D$ & $\mathrm{AL} \quad \mathrm{AD}$ & 35,27 & $\mathrm{~F}^{-}$ & $\mathrm{F}^{-}$ & $\mathrm{F}^{-}$ & $-0,94$ & $-4,43$ & $-0,26$ \\
\hline 102 & $\mathrm{AD}$ & $\mathrm{AD}$ & $\mathrm{AL} \quad \mathrm{AD}$ & $\mathrm{AD} A D$ & 35,53 & $\mathrm{~F}^{-}$ & $\mathrm{F}^{-}$ & $\mathrm{F}^{-}$ & $-0,85$ & $-3,99$ & $-2,12$ \\
\hline 103 & $\mathrm{AD}$ & $\mathrm{AD}$ & $\mathrm{AL} \quad \mathrm{BA}$ & $\mathrm{AD} A D$ & 37,65 & $\mathrm{~F}^{-}$ & $\mathrm{F}^{-}$ & - & 0,00 & $-3,58$ & $-0,59$ \\
\hline 110 & $\mathrm{AD}$ & $\overline{A D}$ & $\mathrm{AD} \mathrm{AD}$ & $\mathrm{AL} \quad \mathrm{AD}$ & 41,82 & $\mathrm{~F}^{-}$ & $\mathrm{F}^{-}$ & $\mathrm{V}^{-}$ & $-1,19$ & $-2,55$ & 2,12 \\
\hline 111 & $\mathrm{AD}$ & $\mathrm{AD}$ & $\mathrm{AL} \quad \mathrm{AD}$ & $\mathrm{AL} A D$ & 39,70 & $\mathrm{~F}^{-}$ & $\mathrm{F}^{-}$ & $\mathrm{V}^{-}$ & $-1,10$ & $-2,12$ & 0,18 \\
\hline 112 & $A D$ & $\mathrm{AD}$ & $\mathrm{AL} \quad \mathrm{AD}$ & $\mathrm{AL} \quad \mathrm{AD}$ & 39,52 & $\mathrm{~F}^{-}$ & $\mathrm{F}^{-}$ & $\mathrm{F}^{-}$ & $-0,94$ & $-1,62$ & $-1,71$ \\
\hline 113 & $\mathrm{AD}$ & $\mathrm{AD}$ & $\mathrm{AL} \quad \mathrm{AD}$ & $\mathrm{AD} A D$ & 41,23 & $\mathrm{~F}^{-}$ & $\mathrm{F}^{-}$ & - & 0,00 & $-1,18$ & $-0,59$ \\
\hline 120 & $\mathrm{AD}$ & $\mathrm{AD}$ & $\mathrm{AD} \quad \mathrm{AD}$ & $\mathrm{AL} \quad \mathrm{AD}$ & 44,37 & $\mathrm{~F}^{-}$ & $\mathrm{F}^{-}$ & $\mathrm{V}^{-}$ & $-1,28$ & $-0,25$ & 2,55 \\
\hline 121 & $\mathrm{AD}$ & $\mathrm{AD}$ & $\mathrm{AL} \quad \mathrm{AD}$ & $\mathrm{AL} \quad \mathrm{AD}$ & 41,82 & $\mathrm{~F}^{-}$ & $\mathrm{V}^{-}$ & $\mathrm{V}^{-}$ & $-1,19$ & 0,26 & 0,68 \\
\hline 122 & $\mathrm{AL}$ & $\mathrm{AD}$ & $\mathrm{AL} \quad \overline{\mathrm{AD}}$ & $\mathrm{AL} A D$ & 41,14 & $\mathrm{~F}^{-}$ & $\mathrm{V}^{-}$ & $\mathrm{F}^{-}$ & $-1,10$ & 0,60 & $-1,27$ \\
\hline 123 & AL & $\mathrm{AD}$ & $\mathrm{AL} \quad \mathrm{AD}$ & $A D A D$ & 42,41 & $\mathrm{~F}^{-}$ & $\mathrm{V}^{-}$ & - & 0,00 & 1,10 & $-0,59$ \\
\hline 130 & $\mathrm{AD}$ & $\mathrm{AD}$ & $\mathrm{AD} \quad \mathrm{AD}$ & $\mathrm{AL} \quad \mathrm{AD}$ & 44,62 & $F^{-}$ & - & $\mathrm{V}^{-}$ & 0,00 & $-0,43$ & 3,06 \\
\hline 131 & $A D$ & $\mathrm{AD}$ & $\mathrm{AD} A \mathrm{AD}$ & $\mathrm{AL} \quad \mathrm{AD}$ & 41,56 & $\mathrm{~F}^{-}$ & - & $\mathrm{V}^{-}$ & 0,00 & 0,08 & 1,02 \\
\hline 132 & $\mathrm{AL}$ & $\mathrm{AD}$ & $\mathrm{AL} \quad \mathrm{AD}$ & $\mathrm{AL} \quad \mathrm{AD}$ & 40,54 & $\mathrm{~F}^{-}$ & - & $\mathrm{F}^{-}$ & 0,00 & 0,51 & $-0,77$ \\
\hline 133 & $\mathrm{AL}$ & $\mathrm{AD}$ & $\mathrm{AL} \quad \mathrm{AD}$ & $\mathrm{AD} \mathrm{AD}$ & 41,31 & $F^{-}$ & - & - & 0,00 & 0,94 & $-0,59$ \\
\hline
\end{tabular}


Apêndice 50. Classificação dos teores foliares segundo o Critério de Faixas de Suficiência, faixas propostas pelo Grupo Paulista de Adubação e Calagem para Citros (1994), diagnose para a correção de N, P e K, resposta líquida da produtividade, diagnose para o nutriente deficiente e a resposta da produtividade, para os dados, dos tratamentos com as doses 2 e 3 de $\mathrm{N}$ em todas as combinações de doses de $\mathrm{P}$ e $\mathrm{K}$, do ensaio de adubação $\mathrm{N}, \mathrm{P}$ e $\mathrm{K}$ conduzido em Matão/SP.

\begin{tabular}{|c|c|c|c|c|c|c|c|c|c|c|c|c|c|}
\hline \multirow[b]{2}{*}{ Trat. } & \multicolumn{6}{|c|}{ Classificacão pelo CFS } & \multirow[b]{2}{*}{ Prod. } & \multicolumn{3}{|c|}{ Diagnose } & \multicolumn{3}{|c|}{ Resposta } \\
\hline & $\mathrm{N}$ & $\mathrm{P}$ & $\mathrm{K}$ & $\mathrm{Ca}$ & $\mathrm{Mg}$ & $\bar{S}$ & & $\mathrm{~N}$ & $\mathrm{P}$ & $\mathrm{K}$ & $\mathrm{d}\left(\mathrm{Y}_{\mathrm{N}}\right)$ & $\mathrm{d}\left(\mathrm{Y}_{\mathrm{P}}\right)$ & $d\left(Y_{K}\right)$ \\
\hline & & & & & & & $\mathrm{tha}^{-1}$ & & & & & $t \mathrm{tha}^{-1}$ & \\
\hline 200 & $\mathrm{AL}$ & $A D$ & $A D$ & $\mathrm{AD}$ & $\mathrm{AL}$ & $\mathrm{AD}$ & 37,99 & $\mathrm{~V}^{-}$ & $\mathrm{F}^{-}$ & $\mathrm{V}^{*}$ & 0,00 & $-5,02$ & 1,78 \\
\hline 201 & $\mathrm{AL}$ & $A D$ & $A D$ & $A D$ & $\mathrm{AL}$ & $\mathrm{AD}$ & 36,21 & $\mathrm{~V}^{-}$ & $F^{-}$ & $\mathrm{F}^{-}$ & 0,09 & $-4,59$ & $-0,17$ \\
\hline 202 & $\mathrm{AL}$ & $\mathrm{AD}$ & $\mathrm{AL}$ & $\mathrm{AD}$ & $\mathrm{AL}$ & $\mathrm{AD}$ & 36,38 & $\mathrm{~V}^{-}$ & $\mathrm{F}^{-}$ & $\mathrm{F}^{-}$ & 0,26 & $-4,08$ & $-1,96$ \\
\hline 203 & $\mathrm{AL}$ & $\mathrm{AD}$ & $\mathrm{AL}$ & BA & $A D$ & $\mathrm{AD}$ & 38,34 & $\mathrm{~V}^{-}$ & $F^{-}$ & - & 0,00 & $-3,65$ & $-0,59$ \\
\hline 210 & $\mathrm{AL}$ & $\mathrm{AD}$ & $A D$ & $\mathrm{AD}$ & $\mathrm{AL}$ & $\mathrm{AD}$ & 43,01 & $F^{*}$ & $\mathrm{~F}^{-}$ & $\mathrm{V}^{-}$ & $-0,08$ & $-2,64$ & 2,21 \\
\hline 211 & $\mathrm{AL}$ & $\mathrm{AD}$ & $\mathrm{AL}$ & $\mathrm{AD}$ & $\mathrm{AL}$ & $\mathrm{AD}$ & 40,80 & $\mathrm{~V}^{-}$ & $\mathrm{F}^{-}$ & $\mathrm{V}^{-}$ & 0,00 & $-2,21$ & 0,34 \\
\hline 212 & $\mathrm{AL}$ & $\mathrm{AD}$ & $\mathrm{AL}$ & $\mathrm{AD}$ & $\mathrm{AL}$ & $A D$ & 40,46 & $\mathrm{~V}^{-}$ & $F^{-}$ & $F^{-}$ & 0,17 & $-1,78$ & $-1,53$ \\
\hline 213 & $\mathrm{AL}$ & $\mathrm{AD}$ & $\mathrm{AL}$ & $\mathrm{AD}$ & $\mathrm{AD}$ & $\mathrm{AD}$ & 41,99 & $\mathrm{~V}^{-}$ & $F^{-}$ & - & 0,00 & $-1,36$ & $-0,59$ \\
\hline 220 & $\mathrm{AL}$ & $\mathrm{AD}$ & $\mathrm{AD}$ & $\mathrm{AD}$ & $\mathrm{AL}$ & $\mathrm{AD}$ & 45,65 & $\mathrm{~F}^{-}$ & $F^{-}$ & $\mathrm{V}^{-}$ & $-0,16$ & $-0,34$ & 2,64 \\
\hline 221 & $\mathrm{AL}$ & $\mathrm{AD}$ & $\mathrm{AL}$ & $\mathrm{AD}$ & $\mathrm{AL}$ & $\mathrm{AD}$ & 43,01 & $\mathrm{~F}^{*}$ & $\mathbf{V}^{*}$ & $\mathrm{~V}^{-}$ & $-0,08$ & 0,17 & 0,77 \\
\hline 222 & $\mathrm{AL}$ & $\mathrm{AD}$ & $\mathrm{AL}$ & $\mathrm{AD}$ & AL & $\mathrm{AD}$ & 42,24 & $\mathrm{~V}^{-}$ & $\mathrm{V}^{-}$ & $\mathrm{F}^{-}$ & 0,08 & 0,59 & $-1,11$ \\
\hline 223 & AL & $\mathrm{AD}$ & $\mathrm{AL}$ & $\mathrm{AD}$ & $\mathrm{AD}$ & $\mathrm{AD}$ & 43,35 & $\mathrm{~V}^{-}$ & $\mathrm{V}^{-}$ & - & 0,00 & 1,02 & $-0,59$ \\
\hline 230 & $\mathrm{AL}$ & $\mathrm{AD}$ & $\mathrm{AD}$ & $A D$ & $\mathrm{AL}$ & $\mathrm{AD}$ & 45,99 & $\mathrm{~F}^{-}$ & - & $\mathrm{V}^{-}$ & 0,00 & $-0,43$ & 3,15 \\
\hline 231 & AL & $\mathrm{AD}$ & $\mathrm{AL}$ & $\mathrm{AD}$ & $\mathrm{AL}$ & $\mathrm{AD}$ & 42,84 & $\mathrm{~F}^{-}$ & - & $\mathrm{V}^{-}$ & 0,00 & 0,08 & 1,19 \\
\hline 232 & $\mathrm{AL}$ & $\mathrm{AD}$ & $\mathrm{AL}$ & $A D$ & $\mathrm{AL}$ & $\mathrm{AD}$ & 41,65 & $\mathrm{~V}^{-}$ & - & $\mathrm{F}^{-}$ & 0,00 & 0,51 & $-0,68$ \\
\hline 233 & $\mathrm{AL}$ & $\mathrm{AD}$ & $\mathrm{AL}$ & $\mathrm{AD}$ & $\mathrm{AD}$ & $\mathrm{AD}$ & 42,33 & $\mathrm{~V}^{-}$ & - & - & 0,00 & 0,94 & $-0,59$ \\
\hline 300 & $\mathrm{AL}$ & $\mathrm{AD}$ & $\mathrm{AD}$ & $\mathrm{AD}$ & $\mathrm{AL}$ & $\mathrm{AD}$ & 37,99 & - & $\mathrm{F}^{-}$ & $\mathrm{V}^{-}$ & 0,00 & $-5,10$ & 1,87 \\
\hline 301 & $\mathrm{AL}$ & $\mathrm{AD}$ & $\mathrm{AD}$ & $\mathrm{AD}$ & $\mathrm{AL}$ & $\mathrm{AD}$ & 36,12 & - & $\mathrm{F}^{-}$ & $\mathrm{V}^{-}$ & 0,00 & $-4,68$ & 0,00 \\
\hline 302 & $\mathrm{AL}$ & $\mathrm{AD}$ & $\mathrm{AL}$ & $\mathrm{AD}$ & $\mathrm{AL}$ & $\mathrm{AD}$ & 36,12 & - & $\mathrm{F}^{-}$ & $F^{-}$ & 0,00 & $-4,17$ & $-1,87$ \\
\hline 303 & $\mathrm{AL}$ & $\mathrm{AD}$ & $\mathrm{AL}$ & $\mathrm{AD}$ & $A D$ & $\mathrm{AD}$ & 37,99 & - & $\mathrm{F}^{-}$ & - & 0,00 & $-3,74$ & $-0,59$ \\
\hline 310 & $\mathrm{AL}$ & $\mathrm{AD}$ & $\mathrm{AD}$ & $\mathrm{AD}$ & $\mathrm{AL}$ & $\mathrm{AD}$ & 43,09 & - & $\mathrm{F}^{-}$ & $\mathrm{V}^{*}$ & 0,00 & $-2,72$ & 2,29 \\
\hline 311 & $\mathrm{AL}$ & $\mathrm{AD}$ & $\mathrm{AL}$ & $\mathrm{AD}$ & $\mathrm{AL}$ & $\mathrm{AD}$ & 40,80 & - & $\mathrm{F}^{-}$ & $\mathrm{V}^{-}$ & 0,00 & $-2,29$ & 0,51 \\
\hline 312 & $\mathrm{AL}$ & $\mathrm{AD}$ & $\mathrm{AL}$ & $\mathrm{AD}$ & $\mathrm{AD}$ & $\mathrm{AD}$ & 40,29 & - & $F^{-}$ & $\mathrm{F}^{-}$ & 0,00 & $-1,87$ & $-1,44$ \\
\hline 313 & $\mathrm{AL}$ & $\mathrm{AD}$ & $\mathrm{AL}$ & $\mathrm{AD}$ & $A D$ & $\mathrm{AD}$ & 41,73 & - & $F^{-}$ & - & 0,00 & $-1,45$ & $-0,59$ \\
\hline 320 & $\mathrm{AL}$ & $\mathrm{AD}$ & $A D$ & $\mathrm{AD}$ & $\mathrm{AD}$ & $\mathrm{AD}$ & 45,81 & - & $F^{-}$ & $\mathrm{V}^{-}$ & 0,00 & $-0,43$ & 2,72 \\
\hline 321 & $\mathrm{AL}$ & $\mathrm{AD}$ & $\mathrm{AL}$ & $\mathrm{AD}$ & $\mathrm{AD}$ & $\mathrm{AD}$ & 43,09 & - & $\mathrm{V}^{-}$ & $\mathrm{V}^{-}$ & 0,00 & 0,08 & 0,93 \\
\hline 322 & $\mathrm{AL}$ & $\mathrm{AD}$ & $\mathrm{AL}$ & $\mathrm{AD}$ & $\mathrm{AD}$ & $\mathrm{AD}$ & 42,16 & - & $\mathrm{V}^{-}$ & $\mathrm{F}^{-}$ & 0,00 & 0,51 & $-1,02$ \\
\hline 323 & $\mathrm{AL}$ & $\mathrm{AD}$ & $\mathrm{AL}$ & $\mathrm{AD}$ & $\mathrm{AD}$ & $\mathrm{AD}$ & 43,18 & - & $\mathrm{V}^{-}$ & - & 0,00 & 0,94 & $-0,59$ \\
\hline 330 & $\mathrm{AL}$ & $\mathrm{AD}$ & $\mathrm{AD}$ & $\mathrm{AD}$ & $\mathrm{AD}$ & $\mathrm{AD}$ & 46,24 & - & - & $\mathrm{V}^{-}$ & 0,00 & $-0,43$ & 3,23 \\
\hline 331 & $\mathrm{AL}$ & $\mathrm{AD}$ & $\mathrm{AL}$ & $\mathrm{AD}$ & $\mathrm{AD}$ & $\mathrm{AD}$ & 43,01 & - & - & $\mathrm{V}^{-}$ & 0,00 & 0,08 & 1,36 \\
\hline 332 & $\mathrm{AL}$ & $\mathrm{AD}$ & $\mathrm{AL}$ & $\mathrm{AD}$ & $\mathrm{AD}$ & $\mathrm{AD}$ & 41,65 & - & - & $F^{-}$ & 0,00 & 0,51 & $-0,59$ \\
\hline 333 & $\mathrm{AL}$ & $\mathrm{AD}$ & $\mathrm{AL}$ & $\mathrm{AD}$ & $\mathrm{AD}$ & $\mathrm{AD}$ & 42,24 & - & - & - & 0,00 & 0,94 & $-0,59$ \\
\hline
\end{tabular}


Apêndice 51. Classificação dos teores foliares segundo o Critério de Faixas de Suficiência, faixas propostas pelo Grupo Paulista de Adubação e Calagem para Citros (1994), diagnose para a correção de $\mathrm{N}, \mathrm{P}$ e K, resposta líquida da produtividade, diagnose para o nutriente deficiente e a resposta da produtividade, para os dados, dos tratamentos com as doses 0 e 1 de $\mathrm{N}$ em todas as combinações de doses de $\mathrm{P}$ e $\mathrm{K}$, do ensaio de adubação $\mathrm{N}, \mathrm{P}$ e $\mathrm{K}$ conduzido em Monte Azul/SP.

\begin{tabular}{|c|c|c|c|c|c|c|c|c|c|c|c|c|c|}
\hline \multirow[b]{2}{*}{ Trat. } & \multicolumn{6}{|c|}{ Classificacão pelo CFS } & \multirow[b]{2}{*}{ Prod. } & \multicolumn{3}{|c|}{ Diagnose } & \multicolumn{3}{|c|}{ Resposta } \\
\hline & $\mathrm{N}$ & $\mathrm{P}$ & $\mathrm{K}$ & $\mathrm{Ca}$ & $\mathrm{Mg}$ & $\bar{S}$ & & $\mathrm{~N}$ & $\mathrm{P}$ & $\mathrm{K}$ & $\overline{d\left(Y_{N}\right)}$ & $\mathrm{d}\left(\mathrm{Y}_{\mathrm{P}}\right)$ & $\overline{d\left(Y_{K}\right)}$ \\
\hline & & & & & & & $t h a^{-1}$ & & & & & tha & \\
\hline 000 & $\mathrm{AD}$ & $A D$ & $A D$ & $A D$ & $\mathrm{AD}$ & $A D$ & 20,00 & $\mathrm{~F}^{-}$ & $\mathrm{V}^{-}$ & $\mathrm{V}^{-}$ & $-6,53$ & 0,42 & 1,25 \\
\hline 001 & $\mathrm{AD}$ & $\mathrm{AD}$ & $\mathrm{AD}$ & $\mathrm{AD}$ & BA & $\mathrm{AD}$ & 18,75 & $\mathrm{~F}^{-}$ & $\mathrm{V}^{-}$ & $\mathrm{V}^{-}$ & $-6,27$ & 0,17 & 0,17 \\
\hline 002 & $\mathrm{AD}$ & $\mathrm{AD}$ & $\mathrm{AD}$ & $\mathrm{AD}$ & $\mathrm{AD}$ & $\mathrm{AD}$ & 18,58 & $F^{-}$ & $\mathrm{F}^{-}$ & $\mathrm{F}^{-}$ & $-6,11$ & $-0,17$ & $-0,92$ \\
\hline 003 & $\mathrm{AD}$ & $A D$ & $\mathrm{AD}$ & $\mathrm{AD}$ & $\mathrm{AD}$ & $\mathrm{AD}$ & 19,50 & $\mathrm{~F}^{-}$ & $F^{-}$ & - & 0,00 & $-0,42$ & $-1,09$ \\
\hline 010 & $\mathrm{AD}$ & $\mathrm{AD}$ & $\mathrm{AD}$ & $\mathrm{AD}$ & $\mathrm{AD}$ & $\mathrm{AD}$ & 19,58 & $\mathrm{~F}^{-}$ & $\mathrm{F}^{-}$ & $\mathrm{V}^{-}$ & $-6,20$ & $-0,26$ & 1,00 \\
\hline 011 & $\mathrm{AD}$ & $A D$ & $\mathrm{AD}$ & $\mathrm{AD}$ & $\mathrm{AD}$ & $\mathrm{AD}$ & 18,58 & $\mathrm{~F}^{*}$ & $F^{-}$ & $\mathrm{F}^{-}$ & $-5,94$ & $-0,59$ & $-0,17$ \\
\hline 012 & $\mathrm{AD}$ & $A D$ & $\mathrm{AD}$ & $\mathrm{AD}$ & $\mathrm{AD}$ & $\mathrm{AD}$ & 18,75 & $\mathrm{~F}^{-}$ & $\mathrm{F}^{-}$ & $\mathrm{F}^{-}$ & $-5,69$ & $-0,83$ & $-1,17$ \\
\hline 013 & $\mathrm{AD}$ & $\mathrm{AD}$ & $\mathrm{AD}$ & $\mathrm{AD}$ & $\mathrm{AD}$ & $\mathrm{AD}$ & 19,92 & $F^{-}$ & $F^{-}$ & - & 0,00 & $-1,17$ & $-1,09$ \\
\hline 020 & $\mathrm{AD}$ & $\overline{\mathrm{AL}}$ & $\mathrm{AD}$ & $\mathrm{AD}$ & $\overline{A D}$ & $\overline{\mathrm{AD}}$ & 19,84 & $\mathrm{~F}^{-}$ & $\mathrm{F}^{-}$ & $\mathrm{V}^{-}$ & $-5,85$ & $-1,00$ & 0,67 \\
\hline 021 & $\mathrm{AD}$ & $A D$ & $\mathrm{AD}$ & $\mathrm{AD}$ & $\mathrm{AD}$ & $\mathrm{AD}$ & 19,17 & $F^{-}$ & $F^{-}$ & $\mathrm{F}^{-}$ & $-5,52$ & $-1,25$ & $-0,41$ \\
\hline 022 & $\mathrm{AD}$ & $\mathrm{AD}$ & $\mathrm{AD}$ & $\mathrm{AD}$ & $\mathrm{AD}$ & $\mathrm{AD}$ & 19,58 & $\mathrm{~F}^{-}$ & $F^{-}$ & $\mathrm{F}^{-}$ & $-5,28$ & $-1,51$ & $-1,51$ \\
\hline 023 & $\mathrm{AD}$ & $\mathrm{AD}$ & $\mathrm{AD}$ & $\mathrm{AD}$ & $\mathrm{AD}$ & $\mathrm{AD}$ & 21,09 & $F^{-}$ & $\mathrm{F}^{-}$ & - & 0,00 & $-1,76$ & $-1,09$ \\
\hline 030 & $\mathrm{AD}$ & $\mathrm{AL}$ & $\mathrm{AD}$ & $\mathrm{AD}$ & $A D$ & $\mathrm{AD}$ & 20,84 & $F^{-}$ & - & $\mathrm{V}^{-}$ & 0,00 & 0,17 & 0,42 \\
\hline 031 & $\mathrm{AD}$ & $\mathrm{AL}$ & $\mathrm{AD}$ & $\mathrm{AD}$ & $\mathrm{AD}$ & $\mathrm{AD}$ & 20,42 & $F^{*}$ & - & $\mathrm{F}^{-}$ & 0,00 & $-0,17$ & $-0,67$ \\
\hline 032 & $\mathrm{AD}$ & $\mathrm{AD}$ & $\mathrm{AD}$ & $A D$ & $\mathrm{AD}$ & $\mathrm{AD}$ & 21,09 & $F^{-}$ & - & $\mathrm{F}^{-}$ & 0,00 & $-0,42$ & $-1,76$ \\
\hline 033 & $\mathrm{AD}$ & $A D$ & $\mathrm{AL}$ & $\mathrm{AD}$ & $\mathrm{AD}$ & $\mathrm{AD}$ & 22,85 & $F^{-}$ & - & - & 0,00 & $-0,67$ & $-1,09$ \\
\hline 100 & $\mathrm{AD}$ & $A D$ & $\mathrm{AD}$ & $\mathrm{AD}$ & $\mathrm{AD}$ & $\mathrm{AD}$ & 26,53 & $F^{*}$ & $\mathrm{~V}^{*}$ & $\mathrm{~V}^{*}$ & $-3,93$ & 0,75 & 1,51 \\
\hline 101 & $A D$ & $A D$ & $A D$ & $\mathrm{AD}$ & BA & $\mathrm{AD}$ & 25,02 & $F^{-}$ & $\mathbf{V}^{-}$ & $\mathrm{V}^{-}$ & $-3,69$ & 0,50 & 0,33 \\
\hline 102 & $\mathrm{AD}$ & $\mathrm{AD}$ & $\mathrm{AD}$ & $\mathrm{AD}$ & $\mathrm{AD}$ & $\mathrm{AD}$ & 24,69 & $F^{-}$ & $\mathrm{V}^{-}$ & $F^{-}$ & $-3,43$ & 0,25 & $-0,67$ \\
\hline 103 & $\mathrm{AD}$ & $A D$ & $\mathrm{AD}$ & $\mathrm{AD}$ & $\mathrm{AD}$ & $\mathrm{AD}$ & 25,36 & $F^{-}$ & $F^{-}$ & - & 0,00 & $-0,08$ & $-1,09$ \\
\hline 110 & $\mathrm{AD}$ & $A D$ & $\mathrm{AD}$ & $\mathrm{AD}$ & $\mathrm{AD}$ & $\mathrm{AD}$ & 25,78 & $F^{-}$ & $\mathrm{V}^{-}$ & $\mathrm{V}^{-}$ & $-3,51$ & 0,09 & 1,26 \\
\hline 111 & $\mathrm{AD}$ & $\mathrm{AD}$ & $\mathrm{AD}$ & $\mathrm{AD}$ & $\mathrm{AD}$ & $\mathrm{AD}$ & 24,52 & $\mathrm{~F}^{-}$ & $F$ & $\mathrm{~V}^{-}$ & $-3,27$ & $-0,17$ & 0,08 \\
\hline 112 & $\mathrm{AD}$ & $A D$ & $\mathrm{AD}$ & $\mathrm{AD}$ & $A D$ & $\mathrm{AD}$ & 24,44 & $F^{*}$ & $F^{-}$ & $F^{-}$ & $-3,01$ & $-0,42$ & $-1,00$ \\
\hline 113 & $\mathrm{AD}$ & $A D$ & $\mathrm{AD}$ & $\mathrm{AD}$ & $\mathrm{AD}$ & $A D$ & 25,44 & $\mathrm{~F}^{-}$ & $F^{-}$ & - & 0,00 & $-0,67$ & $-1,09$ \\
\hline 120 & $\mathrm{AD}$ & $\mathrm{AD}$ & $\mathrm{AD}$ & $\mathrm{AD}$ & $\mathrm{AD}$ & $\mathrm{AD}$ & 25,69 & $F^{-}$ & $F^{-}$ & $\mathrm{V}^{-}$ & $-3,10$ & $-0,59$ & 1,00 \\
\hline 121 & $A D$ & $A D$ & $A D$ & $A D$ & $\mathrm{AD}$ & $A D$ & 24,69 & $F^{-}$ & $F^{-}$ & $F^{-}$ & $-2,93$ & $-0,92$ & $-0,17$ \\
\hline 122 & $\mathrm{AD}$ & $\mathrm{AD}$ & $\mathrm{AD}$ & $\mathrm{AD}$ & $\mathrm{AD}$ & $\mathrm{AD}$ & 24,86 & $\mathrm{~F}$ & $F^{-}$ & $\mathrm{F}^{-}$ & $-2,67$ & $-1,17$ & $-1,25$ \\
\hline 123 & $A D$ & $\mathrm{AD}$ & $\mathrm{AD}$ & $\mathrm{AD}$ & $\mathrm{AD}$ & $\mathrm{AD}$ & 26,11 & $\mathrm{~F}^{*}$ & $F^{-}$ & - & 0,00 & $-1,42$ & $-1,09$ \\
\hline 130 & $\mathrm{AD}$ & $A D$ & $\mathrm{AD}$ & $\mathrm{AD}$ & $\mathrm{AD}$ & $\mathrm{AD}$ & 26,28 & $F^{-}$ & - & $\mathrm{V}^{-}$ & 0,00 & 0,17 & 0,67 \\
\hline 131 & $A D$ & $\mathrm{AD}$ & $\mathrm{AD}$ & $\mathrm{AD}$ & $\mathrm{AD}$ & $\mathrm{AD}$ & 25,61 & $F^{-}$ & - & $F^{-}$ & 0,00 & $-0,17$ & $-0,42$ \\
\hline 132 & $\mathrm{AD}$ & $A D$ & $\mathrm{AD}$ & $\mathrm{AD}$ & $\mathrm{AD}$ & $\mathrm{AD}$ & 26,03 & $\mathrm{~F}^{*}$ & - & $F^{-}$ & 0,00 & $-0,42$ & $-1,50$ \\
\hline 133 & $\mathrm{AD}$ & $A D$ & $\mathrm{AL}$ & $\mathrm{AD}$ & $\mathrm{AD}$ & $\mathrm{AD}$ & 27,53 & $\mathrm{~F}^{-}$ & - & - & 0,00 & $-0,67$ & $-1,09$ \\
\hline
\end{tabular}


Apêndice 52. Classificação dos teores foliares segundo o Critério de Faixas de Suficiência, faixas propostas pelo Grupo Paulista de Adubação e Calagem para Citros (1994), diagnose para a correção de $\mathrm{N}, \mathrm{P}$ e K, resposta líquida da produtividade, diagnose para o nutriente deficiente e a resposta da produtividade, para os dados, dos tratamentos com as doses 2 e 3 de $\mathrm{N}$ em todas as combinações de doses de $P$ e $K$, do ensaio de adubação $N, P$ e $K$ conduzido em Monte Azul/SP.

\begin{tabular}{|c|c|c|c|c|c|c|c|c|c|c|c|c|c|}
\hline \multirow[b]{2}{*}{ Trat. } & \multicolumn{6}{|c|}{ Classificação pelo CFS } & \multirow[b]{2}{*}{ Prod. } & \multicolumn{3}{|c|}{ Diagnose } & \multicolumn{3}{|c|}{ Resposta } \\
\hline & $\mathrm{N}$ & $P$ & $\mathrm{~K}$ & $\mathrm{Ca}$ & $\mathrm{Mg}$ & $\bar{S}$ & & $\mathrm{~N}$ & $\mathrm{P}$ & $\mathrm{K}$ & $\mathrm{d}\left(\mathrm{Y}_{\mathrm{N}}\right)$ & $d\left(Y_{P}\right)$ & $\mathrm{d}\left(\mathrm{Y}_{\mathrm{K}}\right)$ \\
\hline & & & & & & & $\mathrm{tha}^{-1}$ & & & & & $-\mathrm{tha}^{-1}$ & \\
\hline 200 & $\mathrm{AD}$ & $\mathrm{AD}$ & $\mathrm{AD}$ & $\mathrm{AD}$ & $\mathrm{AD}$ & $\mathrm{AD}$ & 30,46 & $F^{-}$ & $\mathrm{V}^{-}$ & $\mathrm{V}^{-}$ & $-1,26$ & 1,17 & 1,75 \\
\hline 201 & $\mathrm{AD}$ & $\mathrm{AD}$ & $\mathrm{AD}$ & $\mathrm{AD}$ & $\mathrm{AD}$ & $A D$ & 28,71 & $\mathrm{~F}^{-}$ & $\mathrm{V}^{-}$ & $\mathrm{V}^{-}$ & $-1,00$ & 0,92 & 0,59 \\
\hline 202 & $\mathrm{AD}$ & $\mathrm{AD}$ & $\mathrm{AD}$ & $\mathrm{AD}$ & $\mathrm{AD}$ & $\mathrm{AD}$ & 28,12 & $\mathrm{~F}^{-}$ & $\mathrm{V}^{-}$ & $\mathrm{F}^{-}$ & $-0,75$ & 0,67 & $-0,42$ \\
\hline 203 & $\mathrm{AD}$ & $\mathrm{AD}$ & $\mathrm{AD}$ & $\mathrm{AD}$ & $\mathrm{AD}$ & $\mathrm{AD}$ & 28,54 & $F^{*}$ & $\mathrm{~V}^{-}$ & - & 0,00 & 0,34 & $-1,09$ \\
\hline 210 & $\mathrm{AD}$ & $\mathrm{AD}$ & $\mathrm{AD}$ & $\mathrm{AD}$ & $\mathrm{AD}$ & $\mathrm{AD}$ & 29,29 & $F^{-}$ & $\mathrm{V}^{-}$ & $\mathrm{V}^{-}$ & $-0,84$ & 0,50 & 1,50 \\
\hline 211 & $\mathrm{AD}$ & $\mathrm{AD}$ & $A D$ & $\mathrm{AD}$ & $\mathrm{AD}$ & $A D$ & 27,79 & $\mathrm{~F}^{-}$ & $\mathrm{V}^{-}$ & $\mathrm{V}^{-}$ & $-0,67$ & 0,17 & 0,34 \\
\hline 212 & $\mathrm{AD}$ & $\mathrm{AD}$ & $\mathrm{AD}$ & $A D$ & $\mathrm{AD}$ & $A D$ & 27,45 & $\mathrm{~F}^{*}$ & $F^{*}$ & $\mathrm{~F}^{*}$ & $-0,42$ & $-0,08$ & $-0,75$ \\
\hline 213 & $\mathrm{AD}$ & $\mathrm{AD}$ & $\mathrm{AD}$ & $A D$ & $A D$ & $\mathrm{AD}$ & 28,20 & $F^{-}$ & $F^{-}$ & - & 0,00 & $-0,34$ & $-1,09$ \\
\hline 220 & $A D$ & $\mathrm{AD}$ & $\mathrm{AD}$ & $\mathrm{AD}$ & $A D$ & $\mathrm{AD}$ & 28,79 & $\mathrm{~F}^{-}$ & $F^{-}$ & $\mathrm{V}^{-}$ & $-0,50$ & $-0,25$ & 1,17 \\
\hline 221 & $\mathrm{AD}$ & $\mathrm{AD}$ & $\mathrm{AD}$ & $\mathrm{AD}$ & $\mathrm{AD}$ & $\mathrm{AD}$ & 27,62 & $\mathrm{~F}^{*}$ & $F^{*}$ & $\mathrm{~V}^{-}$ & $-0,25$ & $-0,50$ & 0,09 \\
\hline 222 & $\mathrm{AD}$ & $A D$ & $\mathrm{AD}$ & $\mathrm{AD}$ & $\mathrm{AD}$ & $\mathrm{AD}$ & 27,53 & $\mathrm{~V}^{-}$ & $\mathrm{F}^{-}$ & $F^{-}$ & 0,00 & $-0,84$ & $-1,01$ \\
\hline 223 & $\mathrm{AD}$ & $\mathrm{AD}$ & $\mathrm{AD}$ & $\mathrm{AD}$ & $\mathrm{AD}$ & $A D$ & 28,54 & $\mathrm{~V}^{-}$ & $\mathrm{F}^{-}$ & - & 0,00 & $-1,09$ & $-1,09$ \\
\hline 230 & $\mathrm{AD}$ & $\mathrm{AD}$ & $\mathrm{AD}$ & $\mathrm{AD}$ & $\mathrm{AD}$ & $A D$ & 29,04 & $\mathrm{~F}^{-}$ & - & $\mathrm{V}^{-}$ & 0,00 & 0,17 & 0,92 \\
\hline 231 & $A D$ & $\mathrm{AD}$ & $\mathrm{AD}$ & $A D$ & $\mathrm{AD}$ & $A D$ & 28,12 & $\mathrm{~V}^{-}$ & - & $\mathrm{F}^{*}$ & 0,00 & $-0,17$ & $-0,25$ \\
\hline 232 & $\mathrm{AD}$ & $\mathrm{AD}$ & $\mathrm{AD}$ & $\mathrm{AD}$ & $\mathrm{AD}$ & $\mathrm{AD}$ & 28,37 & $\mathrm{~V}^{-}$ & - & $\mathrm{F}^{-}$ & 0,00 & $-0,42$ & $-1,26$ \\
\hline 233 & $A D$ & $\mathrm{AD}$ & $\mathrm{AD}$ & $\mathrm{AD}$ & $\mathrm{AD}$ & $\mathrm{AD}$ & 29,63 & $\mathrm{~V}^{-}$ & - & - & 0,00 & $-0,67$ & $-1,09$ \\
\hline 300 & $A D$ & $A D$ & $\mathrm{AD}$ & $\mathrm{AD}$ & $\mathrm{AD}$ & $\mathrm{AD}$ & 31,72 & - & $\mathrm{V}^{-}$ & $\mathrm{V}^{-}$ & 0,00 & 1,59 & 2,01 \\
\hline 301 & $\mathrm{AD}$ & $\mathrm{AD}$ & $\mathrm{AD}$ & $\mathrm{AD}$ & $\mathrm{AD}$ & $\mathrm{AD}$ & 29,71 & - & $\mathrm{V}^{-}$ & $\mathrm{V}^{-}$ & 0,00 & 1,25 & 0,84 \\
\hline 302 & $\mathrm{AD}$ & $\mathrm{AD}$ & $A D$ & $\mathrm{AD}$ & $\mathrm{AD}$ & $A D$ & 28,87 & - & $\mathrm{V}^{-}$ & $F^{-}$ & 0,00 & 1,00 & $-0,25$ \\
\hline 303 & $A D$ & $\mathrm{AD}$ & $\mathrm{AD}$ & $\mathrm{AD}$ & $\mathrm{AD}$ & $\mathrm{AD}$ & 29,12 & - & $\mathrm{V}^{-}$ & - & 0,00 & 0,75 & $-1,09$ \\
\hline 310 & $\mathrm{AD}$ & $\mathrm{AD}$ & $A D$ & $\mathrm{AD}$ & $A D$ & $A D$ & 30,13 & - & $\mathrm{V}^{*}$ & $\mathrm{~V}^{-}$ & 0,00 & 0,84 & 1,67 \\
\hline 311 & $\mathrm{AD}$ & $\mathrm{AD}$ & $\mathrm{AD}$ & $\mathrm{AD}$ & $\mathrm{AD}$ & $\mathrm{AD}$ & 28,46 & - & $\mathrm{V}^{-}$ & $\mathrm{V}^{-}$ & 0,00 & 0,59 & 0,59 \\
\hline 312 & $\mathrm{AD}$ & $A D$ & $\mathrm{AD}$ & $\mathrm{AD}$ & $\mathrm{AD}$ & $\mathrm{AD}$ & 27,87 & - & $\mathrm{V}^{-}$ & $\mathrm{F}^{-}$ & 0,00 & 0,34 & $-0,50$ \\
\hline 313 & $\mathrm{AD}$ & $\mathrm{AD}$ & $\mathrm{AD}$ & $\mathrm{AD}$ & $\mathrm{AD}$ & $\mathrm{AD}$ & 28,37 & - & $\mathrm{V}^{-}$ & - & 0,00 & 0,00 & $-1,09$ \\
\hline 320 & $\mathrm{AD}$ & $\mathrm{AD}$ & $\mathrm{AD}$ & $\mathrm{AD}$ & $A D$ & $\mathrm{AD}$ & 29,29 & - & $\mathrm{V}^{-}$ & $\mathrm{V}^{-}$ & 0,00 & 0,17 & 1,42 \\
\hline 321 & $\mathrm{AD}$ & $\mathrm{AD}$ & $A D$ & $\mathrm{AD}$ & $\mathrm{AD}$ & $\mathrm{AD}$ & 27,87 & - & $\mathrm{F}^{-}$ & $\mathrm{V}^{-}$ & 0,00 & $-0,17$ & 0,34 \\
\hline 322 & $\mathrm{AD}$ & $\mathrm{AD}$ & $A D$ & $\mathrm{AD}$ & $\mathrm{AD}$ & $\mathrm{AD}$ & 27,53 & - & $\mathrm{F}^{-}$ & $\mathrm{F}^{-}$ & 0,00 & $-0,42$ & $-0,84$ \\
\hline 323 & $\mathrm{AD}$ & $\mathrm{AD}$ & $\mathrm{AD}$ & $\mathrm{AD}$ & $\mathrm{AD}$ & $A D$ & 28,37 & - & $\mathrm{F}^{-}$ & - & 0,00 & $-0,67$ & $-1,09$ \\
\hline 330 & $\mathrm{AD}$ & $\mathrm{AD}$ & $\mathrm{BA}$ & $\mathrm{AD}$ & $\mathrm{AD}$ & $\mathrm{AD}$ & 29,12 & - & - & $\mathrm{F}^{+}$ & 0,00 & 0,17 & $-1,08$ \\
\hline 331 & $\mathrm{AD}$ & $\mathrm{AD}$ & $\mathrm{AD}$ & $\mathrm{AD}$ & $A D$ & $\mathrm{AD}$ & 28,04 & - & - & $\mathrm{V}^{-}$ & 0,00 & $-0,17$ & 0,09 \\
\hline 332 & $\mathrm{AD}$ & $\mathrm{AD}$ & $\mathrm{AD}$ & $\mathrm{AD}$ & $\mathrm{AD}$ & $\mathrm{AD}$ & 27,95 & - & - & $\mathrm{F}^{-}$ & 0,00 & $-0,42$ & $-1,09$ \\
\hline 333 & $\mathrm{AD}$ & $\mathrm{AD}$ & $\mathrm{AD}$ & $\mathrm{AD}$ & $\mathrm{AD}$ & $\mathrm{AD}$ & 29,04 & - & - & - & 0,00 & $-0,67$ & $-1,09$ \\
\hline
\end{tabular}


Apêndice 53. Classificação dos teores foliares segundo o Critério de Faixas de Suficiência, faixas propostas pelo Grupo Paulista de Adubação e Calagem para Citros (1994), diagnose para a correção de $\mathrm{N}, \mathrm{P}$ e K, resposta líquida da produtividade, diagnose para o nutriente deficiente e a resposta da produtividade, para os dados, dos tratamentos com as doses 0 e 1 de $\mathrm{N}$ em todas as combinações de doses de $\mathrm{P}$ e $\mathrm{K}$, do ensaio de adubação $\mathrm{N}, \mathrm{P}$ e $\mathrm{K}$ conduzido em Olímpia/SP.

\begin{tabular}{|c|c|c|c|c|c|c|c|c|c|c|c|c|}
\hline \multirow[b]{2}{*}{ Trat. } & \multicolumn{5}{|c|}{ Classificacão pelo CFS } & \multirow[b]{2}{*}{ Prod. } & \multicolumn{3}{|c|}{ Diagnose } & \multicolumn{3}{|c|}{ Resposta } \\
\hline & $\mathrm{N}$ & $\mathbf{P}$ & $\mathrm{K}$ & $\mathrm{Ca}$ & $\mathrm{Mg}$ & & $\mathrm{N}$ & $\mathbf{P}$ & $\mathbf{K}$ & $d\left(Y_{N}\right)$ & $d\left(Y_{P}\right)$ & $\mathrm{d}\left(\mathrm{Y}_{\mathrm{K}}\right)$ \\
\hline & & & & & & tha ${ }^{-1}$ & & & & & $-\mathrm{tha}^{-1}$ & \\
\hline 000 & $\mathrm{AL}$ & $\mathrm{AD}$ & $\mathrm{AD}$ & BA & $A D A D$ & 26,86 & $\mathrm{~V}^{-}$ & $\mathrm{V}^{-}$ & $\mathrm{V}^{-}$ & 0,25 & 0,77 & 0,77 \\
\hline 001 & $\mathrm{AL}$ & $\mathrm{AD}$ & $\mathrm{AD}$ & BA & $\mathrm{AL} \mathrm{AD}$ & 26,09 & $\mathrm{~V}^{-}$ & $\mathrm{V}^{-}$ & $\mathrm{V}^{-}$ & 0,08 & 0,51 & 0,59 \\
\hline 002 & $\mathrm{AL}$ & $\mathrm{AD}$ & $\mathrm{AD}$ & $\mathrm{BA}$ & $\mathrm{AL} \quad \mathrm{AL}$ & 25,50 & $\mathrm{~V}^{-}$ & $\mathrm{V}^{-}$ & $\mathrm{V}^{-}$ & 0,00 & 0,25 & 0,51 \\
\hline 003 & & $\mathrm{AD}$ & $\mathrm{AD}$ & $\mathrm{BA}$ & $\mathrm{AL} \quad \mathrm{AL}$ & 24,99 & $\mathrm{~F}^{-}$ & $\mathrm{V}^{-}$ & - & 0,00 & 0,00 & 0,86 \\
\hline 010 & $\mathrm{AL}$ & $\mathrm{AD}$ & $\mathrm{AD}$ & BA & $\mathrm{AL} \mathrm{AD}$ & 26,09 & $\mathrm{~V}^{-}$ & $F^{-}$ & $\mathrm{V}^{-}$ & 0,84 & $-0,43$ & 0,51 \\
\hline 011 & $\mathrm{AL}$ & $\mathrm{AD}$ & $\mathrm{AD}$ & BA & $\mathrm{AL} \quad \mathrm{AL}$ & 25,58 & $\mathrm{~V}^{-}$ & $F^{-}$ & $\mathrm{V}^{-}$ & 0,67 & $-0,77$ & 0,33 \\
\hline 012 & $\mathrm{AL}$ & $A D$ & $A D$ & BA & $\mathrm{AL} \quad \mathrm{AL}$ & 25,25 & $\mathrm{~V}^{-}$ & $\mathrm{F}^{-}$ & $\mathrm{V}^{-}$ & 0,52 & $-1,02$ & 0,26 \\
\hline 013 & $\mathrm{AL}$ & $\mathrm{AD}$ & $\mathrm{AD}$ & $\mathrm{BA}$ & $\mathrm{AL} \quad \mathrm{AL}$ & 24,99 & $\mathrm{~V}^{-}$ & $F^{-}$ & - & 0,00 & $-1,36$ & 0,86 \\
\hline 020 & $\mathrm{AL}$ & $A D$ & $\mathrm{AD}$ & BA & $\mathrm{AL} \quad \mathrm{AD}$ & 26,52 & $\mathrm{~V}^{-}$ & $F^{-}$ & $\mathrm{V}^{-}$ & 1,36 & $-1,79$ & 0,17 \\
\hline 021 & $\mathrm{AL}$ & $\mathrm{AD}$ & $\mathrm{AD}$ & BA & $\mathrm{AL} \quad \mathrm{AD}$ & 26,35 & $\mathrm{~V}^{*}$ & $F^{-}$ & $\mathrm{V}^{*}$ & 1,19 & $-2,04$ & 0,08 \\
\hline 022 & $\mathrm{AL}$ & $\mathrm{AD}$ & $\mathrm{AD}$ & BA & $\mathrm{AL} \quad \mathrm{AL}$ & 26,27 & $\mathrm{~V}^{*}$ & $F^{-}$ & $\mathrm{F}^{-}$ & 1,02 & $-2,37$ & $-0,08$ \\
\hline 023 & $\mathrm{AL}$ & $\mathrm{AD}$ & $A D$ & BA & $\mathrm{AL} \quad \mathrm{AL}$ & 26,35 & $\mathrm{~V}^{-}$ & $F^{-}$ & - & 0,00 & $-2,63$ & 0,86 \\
\hline 030 & $\mathrm{AD}$ & $\mathrm{AD}$ & $\mathrm{AD}$ & $\mathrm{BA}$ & $\mathrm{AD} \quad \mathrm{AD}$ & 28,31 & $\mathrm{~V}^{*}$ & - & $\mathrm{F}^{-}$ & 0,00 & $-0,17$ & $-0,08$ \\
\hline 031 & $A D$ & $\mathrm{AD}$ & $\mathrm{AD}$ & BA & $\mathrm{AL} \quad \mathrm{AD}$ & 28,39 & $\mathrm{~V}^{*}$ & - & $\mathrm{F}^{-}$ & 0,00 & $-0,51$ & $-0,25$ \\
\hline 032 & $\mathrm{AL}$ & $A D$ & $\mathrm{AD}$ & $\mathrm{BA}$ & $\mathrm{AL} \quad \mathrm{AD}$ & 28,64 & $\mathrm{~V}^{-}$ & - & $\mathrm{F}^{-}$ & 0,00 & $-0,84$ & $-0,34$ \\
\hline 033 & $\mathrm{AL}$ & $\mathrm{AD}$ & $\mathrm{AD}$ & $\mathrm{BA}$ & $\mathrm{AL} \quad \mathrm{AD}$ & 28,98 & $\mathrm{~V}^{-}$ & - & - & 0,00 & $-1,02$ & 0,86 \\
\hline 100 & $\mathrm{AL}$ & $\mathrm{AD}$ & $\mathrm{AD}$ & BA & $\mathrm{AL} \quad \mathrm{AD}$ & 26,61 & $\mathrm{~F}^{-}$ & $\mathrm{V}^{-}$ & $\mathrm{V}^{-}$ & $-0,76$ & 1,36 & 0,60 \\
\hline 101 & $\mathrm{AL}$ & $\mathrm{AD}$ & $\mathrm{AD}$ & $\mathrm{BA}$ & $\mathrm{AL} \quad \mathrm{AD}$ & 26,01 & $\mathrm{~F}^{*}$ & $\mathrm{~V}^{-}$ & $\mathrm{V}^{-}$ & $-1,02$ & 1,10 & 0,51 \\
\hline 102 & $\mathrm{AL}$ & $\mathrm{AD}$ & $A D$ & BA & $\mathrm{AL} \quad \mathrm{AL}$ & 25,50 & $\mathrm{~F}^{-}$ & $\mathrm{V}^{*}$ & $\mathrm{~V}^{-}$ & $-1,19$ & 0,77 & 0,34 \\
\hline 103 & $\mathrm{AL}$ & $\mathrm{AD}$ & $\mathrm{AD}$ & $\mathrm{BA}$ & $\mathrm{AL} \quad \mathrm{AL}$ & 25,16 & $\mathrm{~F}^{-}$ & $\mathrm{V}^{-}$ & - & 0,00 & 0,43 & 0,86 \\
\hline 110 & $\mathrm{AL}$ & $\mathrm{AD}$ & $A D$ & $\mathrm{BA}$ & $\mathrm{AL} \quad \mathrm{AD}$ & 25,25 & $\mathrm{~F}^{-}$ & $\mathrm{V}^{-}$ & $\mathrm{V}^{-}$ & $-0,25$ & 0,09 & 0,34 \\
\hline 111 & $\mathrm{AL}$ & $A D$ & $\mathrm{AD}$ & $\mathrm{BA}$ & $\mathrm{AL} A L$ & 24,91 & $\mathrm{~F}^{-}$ & $\mathrm{F}^{-}$ & $\mathrm{V}^{-}$ & $-0,51$ & $-0,25$ & 0,18 \\
\hline 112 & $\mathrm{AL}$ & $A D$ & $\mathrm{AD}$ & $\mathrm{BA}$ & $\mathrm{AL} \quad \mathrm{AL}$ & 24,73 & $\mathrm{~F}^{-}$ & $\mathrm{F}^{-}$ & $\mathrm{V}^{-}$ & $-0,69$ & $-0,52$ & 0,00 \\
\hline 113 & $\mathrm{AL}$ & $\mathrm{AD}$ & $\mathrm{AD}$ & $\mathrm{BA}$ & $\mathrm{AL} \quad \mathrm{AL}$ & 24,73 & $\mathrm{~F}^{-}$ & $\mathrm{F}^{-}$ & - & 0,00 & $-0,77$ & 0,86 \\
\hline 120 & $\mathrm{AL}$ & $\mathrm{AD}$ & $\mathrm{AD}$ & $\mathrm{BA}$ & $\mathrm{AL} \quad \mathrm{AD}$ & 25,16 & $\mathrm{~V}^{-}$ & $\mathrm{F}^{*}$ & $\mathrm{~V}^{-}$ & 0,25 & $-1,27$ & 0,00 \\
\hline 121 & $\mathrm{AL}$ & $\mathrm{AD}$ & $\mathrm{AD}$ & $\mathrm{BA}$ & $\mathrm{AL} \quad \mathrm{AL}$ & 25,16 & $\mathrm{~V}^{-}$ & $\mathrm{F}^{-}$ & $\mathrm{F}^{-}$ & 0,08 & $-1,53$ & $-0,09$ \\
\hline 122 & $\mathrm{AL}$ & $\mathrm{AD}$ & $\mathrm{AD}$ & $\mathrm{BA}$ & $\mathrm{AL} \mathrm{AL}$ & 25,25 & $\mathrm{~F}^{-}$ & $F^{-}$ & $\mathrm{F}^{-}$ & $-0,17$ & $-1,86$ & $-0,25$ \\
\hline 123 & $\mathrm{AL}$ & $\mathrm{AD}$ & $\mathrm{AD}$ & $\mathrm{BA}$ & $\mathrm{AL} \quad \mathrm{AD}$ & 25,50 & $\mathrm{~F}^{-}$ & $\mathrm{F}^{-}$ & - & 0,00 & $-2,12$ & 0,86 \\
\hline 130 & $\mathrm{AL}$ & $\mathrm{AD}$ & $\mathrm{AD}$ & BA & $\mathrm{AL} \quad \mathrm{AD}$ & 26,43 & $\mathrm{~V}^{*}$ & - & $\mathrm{F}^{-}$ & 0,00 & $-0,17$ & $-0,26$ \\
\hline 131 & $\mathrm{AL}$ & $\mathrm{AD}$ & $\mathrm{AD}$ & $\mathrm{BA}$ & $\mathrm{AL} \quad \mathrm{AD}$ & 26,69 & $\mathrm{~V}^{-}$ & - & $\mathrm{F}^{-}$ & 0,00 & $-0,51$ & $-0,42$ \\
\hline 132 & $\mathrm{AL}$ & $\mathrm{AD}$ & $\mathrm{AD}$ & BA & $\mathrm{AL} \quad \mathrm{AD}$ & 27,11 & $\mathrm{~V}^{-}$ & - & $\mathrm{F}^{-}$ & 0,00 & $-0,84$ & $-0,51$ \\
\hline 133 & $\mathrm{AL}$ & $\mathrm{AD}$ & $\mathrm{AD}$ & BA & AL $\mathrm{AD}$ & 27,62 & $\mathrm{~V}^{-}$ & - & - & 0,00 & $-1,02$ & 0,86 \\
\hline
\end{tabular}


Apêndice 54. Classificação dos teores foliares segundo o Critério de Faixas de Suficiência, faixas propostas pelo Grupo Paulista de Adubação e Calagem para Citros (1994), diagnose para a correção de $\mathrm{N}, \mathrm{P}$ e $\mathrm{K}$, resposta líquida da produtividade, diagnose para o nutriente deficiente e a resposta da produtividade, para os dados, dos tratamentos com as doses 2 e 3 de $\mathrm{N}$ em todas as combinações de doses de $\mathrm{P}$ e $\mathrm{K}$, do ensaio de adubação $\mathrm{N}, \mathrm{P}$ e K conduzido em Olímpia/SP.

\begin{tabular}{|c|c|c|c|c|c|c|c|c|c|c|c|c|}
\hline \multirow[b]{2}{*}{ Trat. } & \multicolumn{5}{|c|}{ Classificacão pelo CFS } & \multirow[b]{2}{*}{ Prod. } & \multicolumn{3}{|c|}{ Diagnose } & \multicolumn{3}{|c|}{ Resposta } \\
\hline & $\mathrm{N}$ & $\mathrm{P}$ & $\mathrm{K}$ & $\mathrm{Ca}$ & $\mathrm{Mg}$ & & $\mathrm{N}$ & $\mathbf{P}$ & $\mathrm{K}$ & $\overline{d\left(Y_{N}\right)}$ & $\mathrm{d}\left(\mathrm{Y}_{\mathrm{P}}\right)$ & $\mathrm{d}\left(\mathrm{Y}_{\mathrm{K}}\right)$ \\
\hline & & & & & & $t^{h a^{-1}}$ & & & & & $-t_{h a}^{-1}$ & \\
\hline 200 & $\mathrm{AL}$ & $\mathrm{AD}$ & $\mathrm{AD}$ & BA & $\mathrm{AL} \quad \mathrm{AD}$ & 27,37 & $\mathrm{~F}^{-}$ & $\mathrm{V}^{-}$ & $\mathrm{V}^{-}$ & $-1,96$ & 1,87 & 0,34 \\
\hline 201 & $\mathrm{AL}$ & $\mathrm{AD}$ & $\mathrm{AD}$ & BA & $\mathrm{AL} \quad \mathrm{AL}$ & 27,03 & $\mathrm{~F}^{*}$ & $\mathrm{~V}^{-}$ & $\mathrm{V}^{-}$ & $-2,13$ & 1,61 & 0,34 \\
\hline 202 & $\mathrm{AL}$ & $\mathrm{AD}$ & $\mathrm{AD}$ & BA & $\mathrm{AL} \quad \mathrm{AL}$ & 26,69 & $F^{*}$ & $\mathrm{~V}^{-}$ & $\mathrm{V}^{-}$ & $-2,29$ & 1,27 & 0,17 \\
\hline 203 & $\mathrm{AL}$ & $\mathrm{AD}$ & $\mathrm{AD}$ & BA & $\mathrm{AL} \quad \mathrm{AD}$ & 26,52 & $F^{-}$ & $\mathrm{V}^{-}$ & - & 0,00 & 1,02 & 0,86 \\
\hline 210 & $\mathrm{AL}$ & $\mathrm{AD}$ & $A D$ & BA & $\mathrm{AL} \quad \mathrm{AD}$ & 25,50 & $\mathrm{~F}^{-}$ & $\mathrm{V}^{-}$ & $\mathrm{V}^{-}$ & $-1,44$ & 0,59 & 0,08 \\
\hline 211 & $\mathrm{AL}$ & $\mathrm{AD}$ & $\mathrm{AD}$ & BA & $\mathrm{AL} \quad \mathrm{AL}$ & 25,42 & $\mathrm{~F}^{-}$ & $\mathrm{V}^{*}$ & $\mathrm{~V}^{-}$ & $-1,61$ & 0,34 & 0,00 \\
\hline 212 & $\mathrm{AL}$ & $\mathrm{AD}$ & $A D$ & BA & $\mathrm{AL} \quad \mathrm{AL}$ & 25,42 & $\mathrm{~F}^{-}$ & $\mathrm{V}^{-}$ & $\mathrm{F}^{-}$ & $-1,78$ & 0,00 & $-0,08$ \\
\hline 213 & $\mathrm{AL}$ & $\mathrm{AD}$ & $\mathrm{AD}$ & BA & $\mathrm{AL} \quad \mathrm{AD}$ & 25,50 & $F^{-}$ & $\mathrm{F}^{-}$ & - & 0,00 & $-0,34$ & 0,86 \\
\hline 220 & $\mathrm{AL}$ & $\mathrm{AD}$ & $A D$ & BA & $\mathrm{AL} \quad \mathrm{AD}$ & 24,91 & $\mathrm{~F}^{-}$ & $F^{-}$ & $\mathrm{F}^{-}$ & $-0,93$ & $-0,76$ & $-0,17$ \\
\hline 221 & $\mathrm{AL}$ & $\mathrm{AD}$ & $\mathrm{AD}$ & BA & $\mathrm{AL} \quad \mathrm{AL}$ & 25,08 & $\mathrm{~F}^{-}$ & $\mathrm{F}^{-}$ & $\mathrm{F}^{-}$ & $-1,10$ & $-1,01$ & $-0,34$ \\
\hline 222 & $\mathrm{AL}$ & $\mathrm{AD}$ & $\mathrm{AD}$ & BA & $\mathrm{AL} \quad \mathrm{AL}$ & 25,42 & $\mathrm{~F}^{-}$ & $\mathrm{F}^{-}$ & $\mathrm{F}^{-}$ & $-1,19$ & $-1,27$ & $-0,42$ \\
\hline 223 & $\mathrm{AL}$ & $\mathrm{AD}$ & $\mathrm{AD}$ & BA & $\mathrm{AL} \quad \mathrm{AD}$ & 25,84 & $\mathrm{~F}^{-}$ & $\mathrm{F}^{-}$ & - & 0,00 & $-1,61$ & 0,86 \\
\hline 230 & $\mathrm{AL}$ & $\mathrm{AD}$ & $\mathrm{AD}$ & BA & $\mathrm{AL} \quad \mathrm{AD}$ & 25,67 & $\mathrm{~F}^{-}$ & - & $\mathrm{F}^{-}$ & 0,00 & $-0,17$ & $-0,42$ \\
\hline 231 & $\mathrm{AL}$ & $\mathrm{AD}$ & $\mathrm{AD}$ & BA & $\mathrm{AL} \quad \mathrm{AD}$ & 26,09 & $\mathrm{~F}^{-}$ & - & $F^{-}$ & 0,00 & $-0,51$ & $-0,60$ \\
\hline 232 & $\mathrm{AL}$ & $\mathrm{AD}$ & $\mathrm{AD}$ & BA & $\mathrm{AL} \quad \mathrm{AD}$ & 26,69 & $\mathrm{~F}^{-}$ & - & $F^{-}$ & 0,00 & $-0,84$ & $-0,76$ \\
\hline 233 & $\mathrm{AL}$ & $\mathrm{AD}$ & $\mathrm{AD}$ & BA & $\mathrm{AL} \quad \mathrm{AD}$ & 27,45 & $\mathrm{~F}^{-}$ & - & - & 0,00 & $-1,02$ & 0,86 \\
\hline 300 & $\mathrm{AL}$ & $\mathrm{AD}$ & $\mathrm{AD}$ & BA & $\mathrm{AL} \quad \mathrm{AD}$ & 29,33 & - & $\mathrm{V}^{-}$ & $\mathrm{V}^{*}$ & 0,00 & 2,39 & 0,17 \\
\hline 301 & $\mathrm{AL}$ & $\mathrm{AD}$ & $\mathrm{AD}$ & BA & $\mathrm{AL} \quad \mathrm{AL}$ & 29,16 & - & $\mathbf{V}^{-}$ & $\mathrm{V}^{-}$ & 0,00 & 2,13 & 0,18 \\
\hline 302 & $\mathrm{AL}$ & $\mathrm{AD}$ & $\mathrm{AD}$ & BA & $\mathrm{AL} \quad \mathrm{AD}$ & 28,98 & - & $\mathbf{V}^{-}$ & $\mathrm{F}^{-}$ & 0,00 & 1,78 & $-0,09$ \\
\hline 303 & $\mathrm{AL}$ & $\mathrm{AD}$ & $\mathrm{AD}$ & BA & $\mathrm{AD} \quad \mathrm{AD}$ & 29,07 & - & $\mathrm{V}^{-}$ & - & 0,00 & 1,62 & 0,86 \\
\hline 310 & $\mathrm{AL}$ & $\mathrm{AD}$ & $\mathrm{AD}$ & BA & AL AL & 26,94 & - & $\mathrm{V}^{-}$ & $F^{-}$ & 0,00 & 1,10 & $-0,09$ \\
\hline 311 & $\mathrm{AL}$ & $\mathrm{AD}$ & $\mathrm{AD}$ & BA & $\mathrm{AL} \quad \mathrm{AL}$ & 27,03 & - & $\mathbf{V}^{-}$ & $\mathrm{F}^{-}$ & 0,00 & 0,85 & $-0,17$ \\
\hline 312 & AL & $\mathrm{AD}$ & $\mathrm{AD}$ & BA & $\mathrm{AL} \quad \mathrm{AD}$ & 27,20 & - & $\mathrm{V}^{-}$ & $F^{-}$ & 0,00 & 0,59 & $-0,25$ \\
\hline 313 & $\mathrm{AL}$ & $\mathrm{AD}$ & $\mathrm{AD}$ & BA & $\mathrm{AL} \quad \mathrm{AD}$ & 27,45 & - & $\mathrm{V}^{-}$ & - & 0,00 & 0,16 & 0,86 \\
\hline 320 & $\mathrm{AL}$ & $\mathrm{AD}$ & $A D$ & BA & $\mathrm{AL} \quad \mathrm{AD}$ & 25,84 & - & $\mathrm{F}^{-}$ & $\mathrm{F}^{-}$ & 0,00 & $-0,17$ & $-0,34$ \\
\hline 321 & $\mathrm{AL}$ & $\mathrm{AD}$ & $A D$ & BA & $\mathrm{AL} \quad \mathrm{AD}$ & 26,18 & - & $\mathrm{F}^{-}$ & $F^{-}$ & 0,00 & $-0,51$ & $-0,43$ \\
\hline 322 & $\mathrm{AL}$ & $\mathrm{AD}$ & $\mathrm{AD}$ & BA & $\mathrm{AL} \quad \mathrm{AD}$ & 26,61 & - & $\mathrm{F}^{-}$ & $\mathrm{F}^{-}$ & 0,00 & $-0,84$ & $-0,68$ \\
\hline 323 & $\mathrm{AL}$ & $\mathrm{AD}$ & $\mathrm{AD}$ & BA & $\mathrm{AL} \quad \mathrm{AD}$ & 27,29 & - & $\mathrm{F}^{-}$ & - & 0,00 & $-1,02$ & 0,86 \\
\hline 330 & $\mathrm{AL}$ & $\mathrm{AD}$ & BA & BA & $\mathrm{AL} \quad \mathrm{AD}$ & 26,01 & - & - & $\mathrm{V}^{+}$ & 0,00 & $-0,17$ & 0,68 \\
\hline 331 & $\mathrm{AL}$ & $\mathrm{AD}$ & BA & BA & $\mathrm{AL} \quad \mathrm{AD}$ & 26,69 & - & - & $\mathrm{V}^{+}$ & 0,00 & $-0,51$ & 0,76 \\
\hline 332 & $\mathrm{AL}$ & $\mathrm{AD}$ & BA & BA & $\mathrm{AL} \quad \mathrm{AD}$ & 27,45 & - & - & $\mathrm{V}^{+}$ & 0,00 & $-0,84$ & 0,86 \\
\hline 333 & $\mathrm{AL}$ & $\mathrm{AD}$ & $A D$ & BA & $\mathrm{AD} \mathrm{AD}$ & 28,31 & - & - & - & 0,00 & $-1,02$ & 0,86 \\
\hline
\end{tabular}


Apêndice 55. Classificação dos teores foliares segundo o Critério de Faixas de Suficiência, faixas propostas pelo Grupo Paulista de Adubação e Calagem para Citros (1994), diagnose para a correção de $\mathrm{N}, \mathrm{P}$ e K , resposta líquida da produtividade, diagnose para o nutriente deficiente e a resposta da produtividade, para os dados, dos tratamentos com as doses 0 e 1 de $\mathrm{N}$ em todas as combinações de doses de $\mathrm{P}$ e $\mathrm{K}$, do ensaio de adubação $\mathrm{N}, \mathrm{P}$ e $\mathrm{K}$ conduzido em Pirassununga/SP.

\begin{tabular}{|c|c|c|c|c|c|c|c|c|c|c|c|c|c|}
\hline \multirow[b]{2}{*}{ Trat. } & \multicolumn{6}{|c|}{ Classificação pelo CFS } & \multirow[b]{2}{*}{ Prod. } & \multicolumn{3}{|c|}{ Diagnose } & \multicolumn{3}{|c|}{ Resposta } \\
\hline & $\bar{N}$ & $P$ & $\mathrm{~K}$ & $\mathrm{Ca}$ & $\mathrm{Mg}$ & $\bar{S}$ & & $\mathrm{~N}$ & $\mathrm{P}$ & $\bar{K}$ & $\overline{\mathrm{d}\left(\mathrm{Y}_{\mathrm{N}}\right)}$ & $\mathrm{d}\left(\mathrm{Y}_{\mathrm{P}}\right)$ & $\mathrm{d}\left(\mathrm{Y}_{\mathrm{K}}\right)$ \\
\hline & & & & & & & $\mathrm{tha}^{-1}$ & & & & & $\mathrm{tha}^{-1}$ & \\
\hline 000 & $\mathrm{AD}$ & $\mathrm{AD}$ & $\mathrm{AD}$ & $\mathrm{AD}$ & $\mathrm{AL}$ & $\mathrm{AD}$ & 35,50 & $\mathrm{~F}^{-}$ & $\mathrm{V}^{-}$ & $\mathrm{F}^{-}$ & $-1,76$ & 2,18 & $-0,95$ \\
\hline 001 & $\mathrm{AD}$ & $\mathrm{AD}$ & $\mathrm{AD}$ & $\mathrm{AD}$ & $\mathrm{AD}$ & $\mathrm{AD}$ & 36,45 & $\mathrm{~F}^{-}$ & $\mathrm{V}^{-}$ & $\mathrm{V}^{-}$ & $-1,49$ & 1,23 & 0,55 \\
\hline 002 & $\mathrm{AD}$ & $\mathrm{AD}$ & $\mathrm{AD}$ & $\mathrm{AD}$ & $\mathrm{AD}$ & $\mathrm{AD}$ & 35,90 & $\mathrm{~F}^{-}$ & $\mathrm{V}^{-}$ & $\mathrm{V}^{-}$ & $-1,36$ & 0,27 & 1,90 \\
\hline 003 & $\mathrm{AD}$ & $\mathrm{AD}$ & $\mathrm{AD}$ & $\mathrm{AD}$ & $\mathrm{AD}$ & $\mathrm{AD}$ & 34,00 & $\mathrm{~F}^{-}$ & $F^{-}$ & - & 0,00 & $-0,82$ & $-0,68$ \\
\hline 010 & $\mathrm{AD}$ & $\mathrm{AD}$ & $\mathrm{AD}$ & $\mathrm{AD}$ & $\mathrm{AD}$ & $\mathrm{AD}$ & 33,32 & $\mathrm{~F}^{-}$ & $\mathrm{V}^{-}$ & $\mathrm{F}^{-}$ & $-2,58$ & 3,13 & $-1,90$ \\
\hline 011 & $A D$ & $\mathrm{AD}$ & $\mathrm{AD}$ & $\mathrm{AD}$ & $A D$ & $\mathrm{AD}$ & 35,22 & $\mathrm{~F}^{-}$ & $\mathrm{V}^{-}$ & $\mathrm{F}^{-}$ & $-2,45$ & 2,17 & $-0,41$ \\
\hline 012 & $\mathrm{AD}$ & $A D$ & $\mathrm{AD}$ & $\mathrm{AD}$ & $\mathrm{AD}$ & $\mathrm{AD}$ & 35,63 & $F^{-}$ & $\mathrm{V}^{-}$ & $\mathrm{V}^{*}$ & $-2,31$ & 0,95 & 0,81 \\
\hline 013 & $\mathrm{AD}$ & $\mathrm{AD}$ & $A D$ & $A D$ & $A D$ & $\mathrm{AD}$ & 34,82 & $F^{-}$ & $\mathrm{V}^{-}$ & - & 0,00 & 0,00 & $-0,68$ \\
\hline 020 & $\mathrm{AD}$ & $\mathrm{AD}$ & $\mathrm{AD}$ & $\mathrm{AD}$ & $\mathrm{AD}$ & $\mathrm{AD}$ & 30,19 & $\mathrm{~F}^{-}$ & $\mathrm{V}^{-}$ & $F^{-}$ & $-3,54$ & 3,94 & $-2,86$ \\
\hline 021 & $\mathrm{AD}$ & $\mathrm{AD}$ & $\mathrm{AD}$ & $A D$ & $\mathrm{AD}$ & $\mathrm{AD}$ & 33,05 & $F^{-}$ & $\mathrm{V}^{*}$ & $\mathrm{~F}^{-}$ & $-3,40$ & 2,86 & $-1,63$ \\
\hline 022 & $\mathrm{AD}$ & $A D$ & $\mathrm{AD}$ & $\mathrm{AD}$ & $\mathrm{AD}$ & $A D$ & 34,68 & $\mathrm{~F}^{-}$ & $\mathrm{V}^{-}$ & $\mathrm{F}^{-}$ & $-3,13$ & 1,90 & $-0,14$ \\
\hline 023 & $\mathrm{AD}$ & $\mathrm{AD}$ & $\mathrm{AD}$ & $\mathrm{AD}$ & $\mathrm{AD}$ & $\mathrm{AD}$ & 34,82 & $\mathrm{~F}^{-}$ & $\mathrm{V}^{-}$ & - & 0,00 & 0,96 & $-0,68$ \\
\hline 030 & $\mathrm{AD}$ & $\mathrm{AD}$ & $\mathrm{AD}$ & $\mathrm{AD}$ & $\mathrm{AD}$ & $\mathrm{AD}$ & 26,25 & $\mathrm{~F}^{-}$ & - & $\mathrm{F}^{-}$ & 0,00 & 1,22 & $-3,94$ \\
\hline 031 & $\mathrm{AD}$ & $A D$ & $\mathrm{AD}$ & $\mathrm{AD}$ & $\mathrm{AD}$ & $\mathrm{AD}$ & 30,19 & $\mathrm{~F}^{-}$ & - & $\mathrm{F}^{-}$ & 0,00 & 0,27 & $-2,59$ \\
\hline 032 & $\mathrm{AD}$ & $\mathrm{AD}$ & $\mathrm{AD}$ & $\mathrm{AD}$ & $\mathrm{AD}$ & $\mathrm{AD}$ & 32,78 & $\mathrm{~F}^{*}$ & - & $\mathrm{F}^{*}$ & 0,00 & $-0,82$ & $-1,08$ \\
\hline 033 & $A D$ & $A D$ & $A D$ & $\mathrm{AD}$ & $\mathrm{AD}$ & $A D$ & 33,86 & $\mathrm{~F}^{*}$ & - & - & 0,00 & $-1,91$ & $-0,68$ \\
\hline 100 & $\mathrm{AD}$ & $\mathrm{AD}$ & BA & $\mathrm{AL}$ & $\mathrm{AL}$ & $\mathrm{AD}$ & 37,26 & $\mathrm{~F}^{-}$ & $\mathrm{V}^{-}$ & $\mathrm{V}^{+}$ & $-0,28$ & 1,36 & 0,68 \\
\hline 101 & $\mathrm{AD}$ & $\mathrm{AD}$ & $\mathrm{AD}$ & $\mathrm{AL}$ & $\mathrm{AL}$ & $\mathrm{AD}$ & 37,94 & $F^{-}$ & $\mathrm{V}^{*}$ & $\mathrm{~V}^{-}$ & $-0,14$ & 0,27 & 0,68 \\
\hline 102 & $\mathrm{AD}$ & $A D$ & $\mathrm{AD}$ & $\mathrm{AD}$ & $\mathrm{AD}$ & $\mathrm{AD}$ & 37,26 & $\mathrm{~V}^{-}$ & $\mathrm{F}^{-}$ & $\mathrm{V}^{-}$ & 0,00 & $-0,68$ & 2,17 \\
\hline 103 & $A D$ & $\mathrm{AD}$ & $\mathrm{AD}$ & $\mathrm{AD}$ & $\mathrm{AD}$ & $\mathrm{AD}$ & 35,09 & $\mathrm{~V}^{-}$ & $F^{-}$ & - & 0,00 & $-1,77$ & $-0,68$ \\
\hline 110 & $\mathrm{AD}$ & $\mathrm{AD}$ & BA & $\mathrm{AL}$ & $\mathrm{AL}$ & $\mathrm{AD}$ & 35,90 & $\mathrm{~F}^{*}$ & $\mathrm{~V}^{-}$ & $\mathrm{V}^{+}$ & $-1,23$ & 2,17 & 1,77 \\
\hline 111 & $\mathrm{AD}$ & $\mathrm{AD}$ & $\mathrm{AD}$ & $\mathrm{AL}$ & $\mathrm{AL}$ & $A D$ & 37,67 & $\mathrm{~F}^{-}$ & $\mathrm{V}^{-}$ & $\mathrm{F}^{*}$ & $-1,09$ & 1,22 & $-0,27$ \\
\hline 112 & $\mathrm{AD}$ & $\mathrm{AD}$ & $\mathrm{AD}$ & $\mathrm{AD}$ & $\mathrm{AD}$ & $A D$ & 37,94 & $\mathrm{~F}^{-}$ & $\mathrm{V}^{-}$ & $\mathrm{V}^{-}$ & $-0,96$ & 0,13 & 1,08 \\
\hline 113 & $\mathrm{AD}$ & $\mathrm{AD}$ & $\mathrm{AD}$ & $\mathrm{AD}$ & $\mathrm{AD}$ & $\mathrm{AD}$ & 36,86 & $\mathrm{~F}^{-}$ & $\mathrm{F}^{-}$ & - & 0,00 & $-0,95$ & $-0,68$ \\
\hline 120 & $\mathrm{AD}$ & $A D$ & BA & $\mathrm{AL}$ & $\mathrm{AL}$ & $\mathrm{AD}$ & 33,73 & $\mathrm{~F}^{-}$ & $\mathrm{V}^{-}$ & $\mathrm{V}^{+}$ & $-2,17$ & 3,13 & 2,72 \\
\hline 121 & $\mathrm{AD}$ & $\mathrm{AD}$ & $\mathrm{AD}$ & $\mathrm{AL}$ & $\mathrm{AL}$ & $\overline{A D}$ & 36,45 & $\mathrm{~F}^{-}$ & $\mathrm{V}^{-}$ & $\mathrm{F}^{-}$ & $-2,04$ & 2,04 & $-1,36$ \\
\hline 122 & $\mathrm{AD}$ & $\mathrm{AD}$ & $\mathrm{AD}$ & $\mathrm{AD}$ & $\mathrm{AD}$ & $\mathrm{AD}$ & 37,81 & $\mathrm{~F}^{-}$ & $\mathrm{V}^{-}$ & $\mathrm{V}^{-}$ & $-1,77$ & 0,95 & 0,00 \\
\hline 123 & $\mathrm{AD}$ & $\mathrm{AD}$ & $\mathrm{AD}$ & $\mathrm{AD}$ & $\mathrm{AD}$ & $\mathrm{AD}$ & 37,81 & $\mathrm{~F}^{-}$ & $\mathrm{V}^{-}$ & - & 0,00 & 0,00 & $-0,68$ \\
\hline 130 & $\mathrm{AD}$ & $\mathrm{AD}$ & $\mathrm{BA}$ & $\mathrm{AD}$ & $\mathrm{AL}$ & $\mathrm{AD}$ & 30,60 & $\mathrm{~F}^{-}$ & - & $\mathrm{V}^{+}$ & 0,00 & 1,22 & 3,81 \\
\hline 131 & $A D$ & $\mathrm{AD}$ & $\mathrm{AD}$ & $\mathrm{AD}$ & $\mathrm{AL}$ & $\mathrm{AD}$ & 34,41 & $\mathrm{~F}^{-}$ & - & $\mathrm{F}^{-}$ & 0,00 & 0,27 & $-2,45$ \\
\hline 132 & $\mathrm{AD}$ & $\mathrm{AD}$ & $\mathrm{AD}$ & $\mathrm{AD}$ & AL & $\mathrm{AD}$ & 36,86 & $\mathrm{~F}^{*}$ & - & $\mathrm{F}^{*}$ & 0,00 & $-0,82$ & $-0,95$ \\
\hline 133 & $\mathrm{AD}$ & $\mathrm{AD}$ & $\mathrm{AD}$ & $\mathrm{AD}$ & $\mathrm{AL}$ & $\mathrm{AD}$ & 37,81 & $\mathrm{~F}^{-}$ & - & - & 0,00 & $-1,91$ & $-0,68$ \\
\hline
\end{tabular}


Apêndice 56. Classificação dos teores foliares segundo o Critério de Faixas de Suficiência, faixas propostas pelo Grupo Paulista de Adubação e Calagem para Citros (1994), diagnose para a correção de $\mathrm{N}, \mathrm{P}$ e K, resposta líquida da produtividade, diagnose para o nutriente deficiente e a resposta da produtividade, para os dados, dos tratamentos com as doses 2 e 3 de $\mathrm{N}$ em todas as combinaçães de doses de $\mathrm{P}$ e $\mathrm{K}$, do ensaio de adubação $\mathrm{N}, \mathrm{P}$ e $\mathrm{K}$ conduzido em Pirassununga/SP.

\begin{tabular}{|c|c|c|c|c|c|c|c|c|c|c|c|c|}
\hline \multirow[b]{2}{*}{ Trat. } & \multicolumn{5}{|c|}{ Classificacão pelo CFS } & \multirow[b]{2}{*}{ Prod. } & \multicolumn{3}{|c|}{ Diagnose } & \multicolumn{3}{|c|}{ Resposta } \\
\hline & $\mathrm{N}$ & $\mathrm{P}$ & $\mathrm{K}$ & $\mathrm{Ca}$ & $\mathrm{Mg}$ & & $\mathrm{N}$ & $\mathrm{P}$ & $\mathrm{K}$ & $\mathrm{d}\left(\mathrm{Y}_{\mathrm{N}}\right)$ & $\mathrm{d}\left(\mathrm{Y}_{\mathrm{P}}\right)$ & $\mathrm{d}\left(\mathrm{Y}_{\mathrm{K}}\right)$ \\
\hline & & & & & & that & & & & & $-t h a^{-1}$ & \\
\hline 200 & $A D$ & $A D$ & BA & $\mathrm{AL}$ & $\mathrm{AL} \quad \mathrm{AD}$ & 37,54 & $\mathrm{~V}^{-}$ & $\mathrm{V}^{-}$ & $\mathrm{V}^{+}$ & 0,96 & 0,41 & 0,54 \\
\hline 201 & $\mathrm{AD}$ & $\mathrm{AD}$ & $\mathrm{BA}$ & $\mathrm{AL}$ & $\mathrm{AL} \quad \mathrm{AD}$ & 38,08 & $\mathrm{~V}^{*}$ & $\mathrm{~F}^{-}$ & $\mathrm{F}^{+}$ & 1,22 & $-0,68$ & $-0,82$ \\
\hline 202 & $\mathrm{AD}$ & $\mathrm{AD}$ & $\mathrm{AD}$ & $\mathrm{AD}$ & $\mathrm{AL} \quad \mathrm{AD}$ & 37,26 & $\mathrm{~V}^{-}$ & $F^{*}$ & $\mathrm{~V}^{-}$ & 1,36 & $-1,64$ & 2,31 \\
\hline 203 & $\mathrm{AD}$ & $\mathrm{AD}$ & $\mathrm{AD}$ & $\mathrm{AD}$ & $\mathrm{AD} \mathrm{AD}$ & 34,95 & $\mathrm{~V}^{-}$ & $\mathrm{F}^{-}$ & - & 0,00 & $-2,72$ & $-0,68$ \\
\hline 210 & $\mathrm{AD}$ & $\mathrm{AD}$ & BA & $\mathrm{AL}$ & $\mathrm{AL} \quad \mathrm{AD}$ & 37,13 & $\mathrm{~V}^{-}$ & $\mathrm{V}^{-}$ & $\mathrm{V}^{+}$ & 0,00 & 1,23 & 1,63 \\
\hline 211 & $\mathrm{AD}$ & $\mathrm{AD}$ & BA & $\mathrm{AL}$ & $\mathrm{AL} \mathrm{AD}$ & 38,76 & $\mathrm{~V}^{-}$ & $\mathrm{V}^{-}$ & $\mathrm{V}^{+}$ & 0,27 & 0,27 & 0,14 \\
\hline 212 & $\mathrm{AD}$ & $A D$ & $A D$ & $\mathrm{AD}$ & $\mathrm{AL} \quad \mathrm{AD}$ & 38,90 & $\mathrm{~V}^{-}$ & $F^{-}$ & $\mathrm{V}^{-}$ & 0,41 & $-0,68$ & 1,23 \\
\hline 213 & $A D$ & $\mathrm{AD}$ & $\mathrm{AD}$ & $\mathrm{AD}$ & $\mathrm{AD} \mathrm{AD}$ & 37,67 & $\mathrm{~V}^{*}$ & $F^{*}$ & - & 0,00 & $-1,77$ & $-0,68$ \\
\hline 220 & $\mathrm{AD}$ & $\mathrm{AD}$ & $\mathrm{BA}$ & $\mathrm{AL}$ & $\mathrm{AL} \quad \mathrm{AD}$ & 35,90 & $\mathrm{~F}^{-}$ & $\mathrm{V}^{-}$ & $\mathrm{V}^{+}$ & $-0,82$ & 2,17 & 2,59 \\
\hline 221 & $\mathrm{AD}$ & $\mathrm{AD}$ & BA & $\mathrm{AL}$ & $\mathrm{AL} \quad \mathrm{AD}$ & 38,49 & $F^{*}$ & $\mathbf{V}^{*}$ & $\mathrm{~V}^{+}$ & $-0,68$ & 1,23 & 1,09 \\
\hline 222 & $A D$ & $\mathrm{AD}$ & $\mathrm{AD}$ & $\mathrm{AD}$ & $\mathrm{AL} \mathrm{AD}$ & 39,58 & $\mathrm{~F}^{-}$ & $\mathrm{V}^{-}$ & $\mathrm{V}^{-}$ & $-0,54$ & 0,00 & 0,14 \\
\hline 223 & $\mathrm{AD}$ & $\overline{A D}$ & $\overline{A D}$ & $\mathrm{AD}$ & $\mathrm{AL} \mathrm{AD}$ & 39,44 & $\mathrm{~F}^{-}$ & $\mathrm{F}^{-}$ & - & 0,00 & $-0,95$ & $-0,68$ \\
\hline 230 & $\mathrm{AD}$ & $\mathrm{AD}$ & BA & $\mathrm{AL}$ & $\mathrm{AL} \quad \mathrm{AD}$ & 33,73 & $\mathrm{~F}^{-}$ & - & $\mathrm{V}^{+}$ & 0,00 & 1,22 & 3,53 \\
\hline 231 & $\mathrm{AD}$ & $\mathrm{AD}$ & BA & $\mathrm{AD}$ & $\mathrm{AL} \quad \mathrm{AD}$ & 37,26 & $F^{-}$ & - & $\mathrm{V}^{+}$ & 0,00 & 0,27 & 2,32 \\
\hline 232 & $A D$ & $\mathrm{AD}$ & $\mathrm{AD}$ & $\mathrm{AD}$ & $\mathrm{AL} \quad \mathrm{AD}$ & 39,58 & $\mathrm{~F}^{-}$ & - & $\mathrm{F}^{-}$ & 0,00 & $-0,82$ & $-0,81$ \\
\hline 233 & $\mathrm{AD}$ & $\mathrm{AD}$ & $\mathrm{AD}$ & $\mathrm{AD}$ & $\mathrm{AL} \quad \mathrm{AD}$ & 40,39 & $\mathrm{~F}^{-}$ & - & - & 0,00 & $-1,91$ & $-0,68$ \\
\hline 300 & $\mathrm{AD}$ & $\mathrm{AD}$ & BA & $\mathrm{AL}$ & $\mathrm{AL} \quad \mathrm{AD}$ & 36,58 & - & $F^{-}$ & $\mathrm{V}^{+}$ & 0,00 & $-0,55$ & 0,28 \\
\hline 301 & $\mathrm{AD}$ & $\overline{A D}$ & $\mathrm{BA}$ & $\mathrm{AD}$ & $\mathrm{AL} \quad \overline{\mathrm{AD}}$ & 36,86 & - & $\mathrm{F}^{-}$ & $\mathrm{F}^{+}$ & 0,00 & $-1,63$ & $-0,96$ \\
\hline 302 & $\mathrm{AD}$ & $\mathrm{AD}$ & $\mathrm{BA}$ & $\mathrm{AD}$ & $\mathrm{AL} \quad \mathrm{AD}$ & 35,90 & - & $\mathrm{F}^{-}$ & $\mathrm{F}^{+}$ & 0,00 & $-2,59$ & $-2,44$ \\
\hline 303 & $\mathrm{AD}$ & $\mathrm{AD}$ & $\mathrm{BA}$ & $\mathrm{AD}$ & $\mathrm{AL} \quad \mathrm{AD}$ & 33,46 & - & $\mathrm{F}^{-}$ & - & 0,00 & $-3,53$ & $-0,68$ \\
\hline 310 & $A D$ & $\mathrm{AD}$ & BA & $\mathrm{AL}$ & $\mathrm{AL} \quad \mathrm{AD}$ & 37,13 & - & $\mathrm{V}^{-}$ & $\mathrm{V}^{+}$ & 0,00 & 0,41 & 1,36 \\
\hline 311 & $\mathrm{AD}$ & $\mathrm{AD}$ & BA & $\mathrm{AL}$ & $\mathrm{AL} \quad \mathrm{AD}$ & 38,49 & - & $F^{-}$ & $\mathrm{F}^{+}$ & 0,00 & $-0,68$ & 0,00 \\
\hline 312 & $\mathrm{AD}$ & $\mathrm{AD}$ & $\mathrm{BA}$ & $\mathrm{AD}$ & $\mathrm{AL} \mathrm{AD}$ & 38,49 & - & $\mathrm{F}^{-}$ & $\mathrm{F}^{+}$ & 0,00 & $-1,63$ & $-1,50$ \\
\hline 313 & $\mathrm{AD}$ & $\mathrm{AD}$ & $A D$ & $\mathrm{AD}$ & $\mathrm{AL} \mathrm{AD}$ & 36,99 & - & $F^{-}$ & - & 0,00 & $-2,72$ & $-0,68$ \\
\hline 320 & $A D$ & $\mathrm{AD}$ & BA & $\mathrm{AL}$ & $\mathrm{AL} \quad \mathrm{AD}$ & 36,72 & - & $\mathrm{V}^{-}$ & $\mathrm{V}^{+}$ & 0,00 & 1,22 & 2,45 \\
\hline 321 & $A D$ & $A D$ & $\mathrm{BA}$ & $\mathrm{AD}$ & $\mathrm{AL} \mathrm{AD}$ & 39,17 & - & $\mathrm{V}^{-}$ & $\mathrm{V}^{+}$ & 0,00 & 0,27 & 0,95 \\
\hline 322 & $\mathrm{AD}$ & $A D$ & $\mathrm{AD}$ & $\mathrm{AD}$ & $\mathrm{AL} \quad \mathrm{AD}$ & 40,12 & - & $F^{-}$ & $\mathrm{V}^{-}$ & 0,00 & $-0,82$ & 0,41 \\
\hline 323 & $\mathrm{AD}$ & $\mathrm{AD}$ & $\mathrm{AD}$ & $\mathrm{AD}$ & $\mathrm{AL} \quad \mathrm{AD}$ & 39,71 & - & $\mathrm{F}^{-}$ & - & 0,00 & $-1,91$ & $-0,68$ \\
\hline 330 & $\mathrm{AD}$ & $\mathrm{AD}$ & BA & $\mathrm{AL}$ & $\mathrm{AL} \mathrm{AD}$ & 35,50 & - & - & $\mathrm{V}^{+}$ & 0,00 & 1,22 & 3,40 \\
\hline 331 & $\mathrm{AD}$ & $\mathrm{AD}$ & $\mathrm{BA}$ & $\mathrm{AD}$ & $\mathrm{AL} \mathrm{AD}$ & 38,90 & - & - & $\mathrm{V}^{+}$ & 0,00 & 0,27 & 2,04 \\
\hline 332 & $\mathrm{AD}$ & $\mathrm{AD}$ & $\mathrm{AD}$ & $A D$ & $\mathrm{AL} \quad \mathrm{AD}$ & 40,94 & - & - & $F^{-}$ & 0,00 & $-0,82$ & $-0,68$ \\
\hline 333 & $\overline{A D}$ & $\overline{A D}$ & $\mathrm{AD}$ & $\mathrm{AD}$ & $A L A D$ & 41,62 & - & - & - & 0,00 & $-1,91$ & $-0,68$ \\
\hline
\end{tabular}

